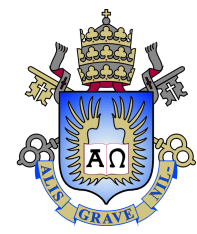

Rocío Mederos Hernández

Study of the Electromagnetic Propagation in Stratified Anisotropic Media via Semi-Analytical Methods

Dissertation presented to the Programa de Pós-graduação em Engenharia Elétrica of PUC-Rio in partial fulfillment of the requirements for the degree of Mestre em Engenharia Elétrica.

Advisor: Prof. Guilherme Simon da Rosa 


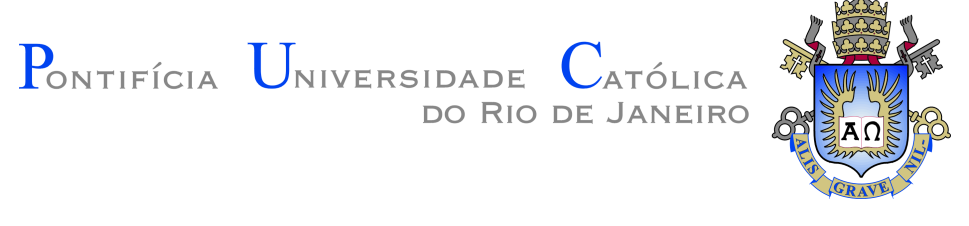

Rocío Mederos Hernández

\section{Study of the Electromagnetic Propagation in Stratified Anisotropic Media via Semi-Analytical Methods}

Dissertation presented to the Programa de Pós-graduação em Engenharia Elétrica of PUC-Rio in partial fulfillment of the requirements for the degree of Mestre em Engenharia Elétrica. Approved by the Examination Committee:

Prof. Guilherme Simon da Rosa

Advisor

Centro de Estudos em Telecomunicações - PUC-Rio

Prof. Maiquel dos Santos Canabarro

UFPel

Prof. José Ricardo Bergmann

Centro de Estudos em Telecomunicações - PUC-Rio

Rio de Janeiro, September the 11th, 2020 
All rights reserved.

\section{Rocío Mederos Hernández}

Received the B.S. degree in Telecommunications and Electronic Engineering from the Technology University of Havana "Jose Antonio Echeverria", The Havana, Cuba, in 2017.

Bibliographic data

Mederos Hernández, Rocío

Study of the Electromagnetic Propagation in Stratified Anisotropic Media via Semi-Analytical Methods / Rocío Mederos Hernández; advisor: Guilherme Simon da Rosa. 2020.

103 f: il. color. ; $30 \mathrm{~cm}$

Dissertação (mestrado) - Pontifícia Universidade Católica do Rio de Janeiro, Departamento de Engenharia Elétrica, 2020.

Inclui bibliografia

1. Engenharia Elétrica - Teses. 2. Meios Anisotrópicos. 3. Meios Estratificados Planares. 4. Método Semianalítico. 5. Propagação de Campos Eletromagnéticos.. I. Simon da Rosa, Guilherme. II. Pontifícia Universidade Católica do Rio de Janeiro. Departamento de Engenharia Elétrica. III. Título. 


\section{Acknowledgments}

Firstly, I would like to thank to my family, and in a very special way to my parents and my sister for always being present despite the distance. They are my reason I wake up stronger every day and I continue this difficult journey without them.

I wish to thank to my husband, Emilio, for being my main support, for walking beside me at all times, for his dedication and unconditional love, simply my best friend.

I am very grateful to my advisor, Professor Guilherme Simon da Rosa, for his wisdom and patience, for sharing his knowledge and teaching me tirelessly, for being the light and backbone of this work. This goal would not have been possible without his unconditional guide. It has been a great pleasure working with him.

I would like to thank to all my friends and colleagues for supporting me in one or another way, especially to Adila, David, Randy, Julio, Dayrene, Adrián, Reinier, Johnes, Yoiz and Pedro.

I want to thank to CETUC and all its professors and workers for giving me a warm welcome and make me feel at home.

I would like to thank to PUC-Rio for the exemption of school fees offered by the Programa de Pós-Graduação em Engenharia Elétrica.

This study was financed in part by the Coordenação de Aperfeiçoamento de Pessoal de Nível Superior - Brasil (CAPES) - Finance Code 001. 


\section{Abstract}

Mederos Hernández, Rocío; Simon da Rosa, Guilherme (Advisor). Study of the Electromagnetic Propagation in Stratified Anisotropic Media via Semi-Analytical Methods. Rio de Janeiro, 2020. 103p. Dissertação de Mestrado - Departamento de Engenharia Elétrica, Pontifícia Universidade Católica do Rio de Janeiro.

Electromagnetic well-logging tools have been subject of interest for many decades due to their applications in oil exploration. In order to obtain accurate formation evaluation, a wide variety of numerical methods have been developed on Computational Electromagnetics. The high cost in terms of computational time and resources of these methods for the spatial discretization procedure is a negative point of these traditional methods. In this work, we will explore new semi-analytical approaches to analyze the propagation of electromagnetic fields in anisotropic media comprising planar layers. We will present a mathematical formulation for the electromagnetic fields due to a solenoid source in terms of a sum of modal eigenfunctions. The proposed method allows the analysis of geophysical scenarios similar to those of the Brazilian Pre-Salt, where high conductivity carbonate rocks are predominant. In addition, the effect of pre- and post-salt formations on electromagnetic waves can be easily incorporated into our model. We will present numerical validation results, which demonstrate the potential of the approach proposed in this work to model geophysical sensors in a computationally robust and efficient way.

\section{Keywords}

Anisotropic Media; Planarly-Layered Media; Semi-Analytical Method; Electromagnetic Field Propagation. 


\section{Resumo}

Mederos Hernández, Rocío; Simon da Rosa, Guilherme. Estudo da Propagação Eletromagnética em Meios Anisotrópicos Estratificados via Métodos Semianalíticos. Rio de Janeiro, 2020. 103p. Dissertação de Mestrado - Departamento de Engenharia Elétrica, Pontifícia Universidade Católica do Rio de Janeiro.

As ferramentas de perfilagem eletromagnética para poços e túneis têm sido objeto de interesse da engenharia por muitas décadas devido às suas aplicações para a exploração de petróleo. A fim de obter uma avaliação precisa de uma formação geofísica, uma ampla variedade de métodos de eletromagnetismo computacional foi desenvolvida. O alto custo em termos de recursos computacionais para o procedimento da discretização espacial é um ponto negativo desses métodos tradicionais. Esta pesquisa tem como objetivo explorar novas abordagens semianalíticas para analisar a propagação de campos eletromagnéticos em meios anisotrópicos compreendendo camadas planares. Apresentaremos uma formulação matemática para os campos eletromagnéticos de uma fonte solenoidal em termos de um somatório de autofunções modais. O método proposto permite a análise de cenários geofísicos análogos aos do Pré-Sal brasileiro, onde rochas carbonáticas de alta condutividade são predominantes. Além disso, o efeito das formações do pré e pós-sal nas ondas eletromagnéticas pode ser facilmente incorporadas no nosso modelo. Apresentaremos resultados numéricos de validação, que demonstram o potencial da abordagem proposta neste trabalho para modelar sensores geofísicos de forma computacionalmente robusta e eficiente.

\section{Palavras-chave}

Meios Anisotrópicos; Meios Estratificados Planares; Método Semianalítico; Propagação de Campos Eletromagnéticos. 


\section{Table of contents}

1 Introduction $\quad 16$

$\begin{array}{lll}1.1 & \text { General Introduction } & 16\end{array}$

$\begin{array}{lll}1.2 & \text { Organization of the Dissertation } & 18\end{array}$

2 Electromagnetic Propagation Overview for Oil Well 20

2.1 Introduction 20

2.2 Underground Media Characterization 20

2.2.1 Physics Fundamentals $\quad 21$

2.2.2 Electromagnetic Characterization 21

2.3 Electromagnetic Logging Tools and Techniques 22

2.4 Computational Electromagnetics 24

3 Electromagnetic Field Theory $\quad 26$

$\begin{array}{lll}3.1 & \text { Introduction } & 26\end{array}$

3.2 Maxwell's equations 26

3.3 Source-Free Axial Electromagnetic Fields 27

3.4 Axial Field Components 31

3.5 Transversal Field Components 33

4 Electromagnetic Fields in Horizontal Stratified Media 36

$\begin{array}{lll}4.1 & \text { Introduction } & 36\end{array}$

4.2 Axial Discontinuities 36

4.2.1 Local Reflection and Transmission Coefficients 36

4.2.2 Generalized Reflection Coefficients 39

4.3 The Guidance Condition 40

4.3.1 Validation via Parallel Plate Waveguide 42

4.3.2 Pole-Free Characteristic Equation 42

4.4 Constant Amplitude $\boldsymbol{A}_{j}^{ \pm}$

4.4.1 Source transmitting to positive $\boldsymbol{z} \quad 44$

4.4.2 Source transmitting to negative $\boldsymbol{z}$

4.5 Numerical Implementation and Analysis 45

4.5.1 Lossless Media 45

4.5.1.1 Homogeneous Isotropic Formation 45

4.5.1.2 Half-Space Isotropic Formation 47

4.5.1.3 Multi-Layer Isotropic Formations 49

4.5.1.4 Multi-Layer Anisotropic Formation 70

$\begin{array}{lll}\text { 4.5.2 Lossy Media } & 71\end{array}$

5 Source Expansion $\quad 84$

$\begin{array}{lll}5.1 & \text { Introduction } & 84\end{array}$

5.2 Modal Field Excitation $\quad 85$

5.3 Current Density 86

5.4 Modal Excitation from an Electric Current Source $\quad 87$

5.5 Numerical Implementation and Analysis 94 
6 Conclusions and Suggestions for Future Research

Bibliography 


\section{List of figures}

Figure 1.1 Solenoidal source within an axially-stratified geophysical formation.

Figure 4.1 Front view of an axially-stratified $N+1$ - layers waveguide ( $z$ in meters). The perfect electric conductor (PEC) is represented by the stripes.

Figure 4.2 Front view of an axially-stratified $N+1$ - layers waveguide. 39

Figure 4.3 Front view of an parallel plate waveguide.

Figure 4.4 Characteristic equation evaluated along the imaginary $k_{\rho^{-}}$-axis.

Figure 4.5 Normalized amplitude of the radial component of the electric field $\left(E_{\rho}\right)$ for decoupled TE and TM modes in a homogeneous and isotropic medium confined by a parallel plate waveguide (First Mode).

Figure 4.6 Normalized amplitude of the radial component of the magnetic field $\left(H_{\rho}\right)$ for decoupled TE and TM modes in a homogeneous and isotropic medium confined by a parallel plate waveguide (First Mode).

Figure 4.7 Normalized amplitude of the azimuthal component of the electric field $\left(E_{\phi}\right)$ for decoupled TE and TM modes in a homogeneous and isotropic medium confined by a parallel plate waveguide (First Mode).

Figure 4.8 Normalized amplitude of the azimuthal component of the magnetic field $\left(H_{\phi}\right)$ for decoupled TE and TM modes in a homogeneous and isotropic medium confined by a parallel plate waveguide (First Mode).

Figure 4.9 Normalized amplitude of the axial component of the electric and magnetic field $\left(E_{z}\right.$ and $\left.H_{z}\right)$ in a homogeneous and isotropic medium confined by a parallel plate waveguide (First Mode).

Figure 4.10 Normalized amplitude of the electric flux density and magnetic flux density $\left(D_{z}\right.$ and $\left.B_{z}\right)$ in a homogeneous and isotropic medium confined by a parallel plate waveguide (First Mode).

Figure 4.11 Normalized amplitude of the radial component of the electric field $\left(E_{\rho}\right)$ for decoupled TE and TM modes in a homogeneous and isotropic medium confined by a parallel plate waveguide (Second Mode).

Figure 4.12 Normalized amplitude of the radial component of the magnetic field $\left(H_{\rho}\right)$ for decoupled TE and TM modes in a homogeneous and isotropic medium confined by a parallel plate waveguide (Second Mode). 
Figure 4.13 Normalized amplitude of the azimuthal component of the electric field $\left(E_{\phi}\right)$ for decoupled TE and TM modes in a homogeneous and isotropic medium confined by a parallel plate waveguide (Second Mode).

Figure 4.14 Normalized amplitude of the azimuthal component of the magnetic field $\left(H_{\phi}\right)$ for decoupled TE and TM modes in a homogeneous and isotropic medium confined by a parallel plate waveguide (Second Mode).

Figure 4.15 Normalized amplitude of the axial component of the electric and magnetic field $\left(E_{z}\right.$ and $\left.H_{z}\right)$ in a homogeneous and isotropic medium confined by a parallel plate waveguide (Second Mode).

Figure 4.16 Normalized amplitude of the electric flux density and magnetic flux density $\left(D_{z}\right.$ and $\left.B_{z}\right)$ in a homogeneous and isotropic medium confined by a parallel plate waveguide (Second Mode).

Figure 4.17 Normalized amplitude of the radial component of the electric field $\left(E_{\rho}\right)$ for decoupled TE and TM modes in a homogeneous and isotropic medium confined by a parallel plate waveguide (Third Mode).

Figure 4.18 Normalized amplitude of the radial component of the magnetic field $\left(H_{\rho}\right)$ for decoupled TE and TM modes in a homogeneous and isotropic medium confined by a parallel plate waveguide (Third Mode).

Figure 4.19 Normalized amplitude of the azimuthal component of the electric field $\left(E_{\phi}\right)$ for decoupled TE and TM modes in a homogeneous and isotropic medium confined by a parallel plate waveguide (Third Mode).

Figure 4.20 Normalized amplitude of the azimuthal component of the magnetic field $\left(H_{\phi}\right)$ for decoupled TE and TM modes in a homogeneous and isotropic medium confined by a parallel plate waveguide (Third Mode).

Figure 4.21 Normalized amplitude of the axial component of the electric and magnetic field $\left(E_{z}\right.$ and $\left.H_{z}\right)$ in a homogeneous and isotropic medium confined by a parallel plate waveguide (Third Mode).

Figure 4.22 Normalized amplitude of the electric flux density and magnetic flux density $\left(D_{z}\right.$ and $\left.B_{z}\right)$ in a homogeneous and isotropic medium confined by a parallel plate waveguide (Third Mode).

Figure 4.23 Axially-stratified two-layers geophysical formation.

Figure 4.24 Normalized amplitude of the radial component of the electric and magnetic field $\left(E_{\rho}\right.$ and $\left.H_{\rho}\right)$ for decoupled TE and TM modes in an axially-stratified two-layers isotropic medium (First Mode). 
Figure 4.25 Normalized amplitude of the azimuthal component of the electric and magnetic field $\left(E_{\phi}\right.$ and $\left.H_{\phi}\right)$ for decoupled TE and TM modes in an axially-stratified two-layers isotropic medium (First Mode).

Figure 4.26 Normalized amplitude of the electric flux density and magnetic flux density $\left(D_{z}\right.$ and $\left.B_{z}\right)$ in axially-stratified twolayers isotropic medium (First Mode).

Figure 4.27 Normalized amplitude of the radial component of the electric field $\left(E_{\rho}\right)$ for decoupled TE and TM modes in an axiallystratified two-layers isotropic medium (Second Mode).

Figure 4.28 Normalized amplitude of the radial component of the magnetic field $\left(H_{\rho}\right)$ for decoupled TE and TM modes in an axially-stratified two-layers isotropic medium (Second Mode).

Figure 4.29 Normalized amplitude of the azimuthal component of the electric field $\left(E_{\phi}\right)$ for decoupled TE and TM modes in an axially-stratified two-layers isotropic medium (Second Mode).

Figure 4.30 Normalized amplitude of the azimuthal component of the magnetic field $\left(H_{\phi}\right)$ for decoupled TE and TM modes in an axially-stratified two-layers isotropic medium (Second Mode).

Figure 4.31 Normalized amplitude of the electric flux density and magnetic flux density $\left(D_{z}\right.$ and $\left.B_{z}\right)$ in axially-stratified twolayers isotropic medium (Second Mode).

Figure 4.32 Normalized amplitude of the radial component of the electric field $\left(E_{\rho}\right)$ for decoupled TE and TM modes in an axiallystratified two-layers isotropic medium (Third Mode).

Figure 4.33 Normalized amplitude of the radial component of the magnetic field $\left(H_{\rho}\right)$ for decoupled TE and TM modes in an axially-stratified two-layers isotropic medium (Third Mode).

Figure 4.34 Normalized amplitude of the azimuthal component of the electric field $\left(E_{\phi}\right)$ for decoupled TE and TM modes in an axially-stratified two-layers isotropic medium (Third Mode).

Figure 4.35 Normalized amplitude of the azimuthal component of the magnetic field $\left(H_{\phi}\right)$ for decoupled TE and TM modes in an axially-stratified two-layers isotropic medium (Third Mode).

Figure 4.36 Normalized amplitude of the electric flux density and magnetic flux density $\left(D_{z}\right.$ and $\left.B_{z}\right)$ in axially-stratified twolayers isotropic medium (Third Mode).

Figure 4.37 Axially-stratified three-layers geophysical formation.

Figure 4.38 Normalized amplitude of the radial component of the electric and magnetic field $\left(E_{\rho}\right.$ and $\left.H_{\rho}\right)$ for decoupled TE and TM modes in an axially-stratified three-layers isotropic medium (First Mode).

Figure 4.39 Normalized amplitude of the electric flux density and magnetic flux density $\left(D_{z}\right.$ and $\left.B_{z}\right)$ in axially-stratified threelayers isotropic medium (First Mode).

Figure 4.40 Normalized amplitude of the radial component of the electric field $\left(E_{\rho}\right)$ for decoupled TE and TM modes in an axiallystratified three-layers isotropic medium (Second Mode). 
Figure 4.41 Normalized amplitude of the radial component of the magnetic field $\left(H_{\rho}\right)$ for decoupled TE and TM modes in an axially-stratified three-layers isotropic medium (Second Mode).

Figure 4.42 Normalized amplitude of the electric flux density and magnetic flux density $\left(D_{z}\right.$ and $\left.B_{z}\right)$ in axially-stratified threelayers isotropic medium (Second Mode).

Figure 4.43 Normalized amplitude of the radial component of the electric field $\left(E_{\rho}\right)$ for decoupled TE and TM modes in an axiallystratified three-layers isotropic medium (Third Mode).

Figure 4.44 Normalized amplitude of the radial component of the magnetic field $\left(H_{\rho}\right)$ for decoupled TE and TM modes in an axially-stratified three-layers isotropic medium (Third Mode).

Figure 4.45 Normalized amplitude of the electric flux density and magnetic flux density $\left(D_{z}\right.$ and $\left.B_{z}\right)$ in axially-stratified threelayers isotropic medium (Third Mode).

Figure 4.46 Normalized amplitude of the electric flux density and magnetic flux density $\left(D_{z}\right.$ and $\left.B_{z}\right)$ in axially-stratified fourlayers isotropic medium (First Mode).

Figure 4.47 Normalized amplitude of the electric flux density and magnetic flux density $\left(D_{z}\right.$ and $\left.B_{z}\right)$ in axially-stratified fourlayers isotropic medium (Second Mode).

Figure 4.48 Normalized amplitude of the electric flux density and magnetic flux density $\left(D_{z}\right.$ and $\left.B_{z}\right)$ in axially-stratified fourlayers isotropic medium (Third Mode).

Figure 4.49 Normalized amplitude of the electric flux density and magnetic flux density $\left(D_{z}\right.$ and $\left.B_{z}\right)$ in axially-stratified five-layers isotropic medium (First Mode).

Figure 4.50 Normalized amplitude of the electric flux density and magnetic flux density $\left(D_{z}\right.$ and $\left.B_{z}\right)$ in axially-stratified five-layers isotropic medium (Second Mode).

Figure 4.51 Normalized amplitude of the electric flux density and magnetic flux density $\left(D_{z}\right.$ and $\left.B_{z}\right)$ in axially-stratified five-layers isotropic medium (Third Mode).

Figure 4.52 Normalized amplitude of the electric flux density and magnetic flux density $\left(D_{z}\right.$ and $\left.B_{z}\right)$ in axially-stratified five-layers anisotropic medium (First Mode).

Figure 4.53 Normalized amplitude of the electric flux density and magnetic flux density $\left(D_{z}\right.$ and $\left.B_{z}\right)$ in axially-stratified five-layers anisotropic medium (Second Mode).

Figure 4.54 Normalized amplitude of the electric flux density and magnetic flux density $\left(D_{z}\right.$ and $\left.B_{z}\right)$ in axially-stratified five-layers anisotropic medium (Third Mode).

Figure 4.55 Eigenvalue behavior to several conductivity values for TE mode.

Figure 4.56 Non-Variation of the magnetic flux density $\left(B_{z}\right)$ to different two conductivity values in axially-stratified five-layers isotropic medium (Second Mode). 
Figure 4.57 Eigenvalue behavior to several conductivity values for TM mode.

Figure 4.58 Behavior of the first eigenvalue to several conductivity values for TM mode. (Zoom of the eigenvalue 1 from Fig. 4.57).

Figure 4.59 Behavior of the second eigenvalue to several conductivity values for TM mode. (Zoom of the eigenvalue 2 from Fig. 4.57). 80

re 4.60 Behavior of the third eigenvalue to several conductivity values for TM mode. (Zoom of the eigenvalue 3 from Fig. 4.57).

Figure 4.61 Behavior of the fourth eigenvalue to several conductivity values for TM mode. (Zoom of the eigenvalue 4 from Fig. 4.57).

Figure 4.62 Behavior of the fifth eigenvalue to several conductivity values for TM mode. (Zoom of the eigenvalue 5 from Fig. 4.57).

Figure 4.63 Variation of the electric flux density $\left(D_{z}\right)$ to several conductivity values in axially-stratified five-layers isotropic medium (Second Mode).

Figure 5.1 Electric current horizontal source within an axiallystratified geophysical formation.

Figure 5.2 Transmitting tilted-coil antenna with current density $\mathbf{J}_{T}$ [1].

Figure 5.3 Absolute values for 10, 20 and 30 modes of the azimuthal component of the electric field $\left(E_{\phi}\right)$ for TE mode in a lossy homogeneous and isotropic medium confined by a parallel plate waveguide.

Figure 5.4 Absolute values Amplitude for 30, 40 and 50 modes of the azimuthal component of the electric field $\left(E_{\phi}\right)$ for TE mode in a lossy homogeneous and isotropic medium confined by a parallel plate waveguide. 


\section{List of tables}

Table 4.1 Eigenvalues for a parallel plate guide.

Table 4.2 Physical characteristics of an axially-stratified two-layers isotropic medium.

Table 4.3 Eigenvalues for an axially-stratified two-layers isotropic medium.

Table 4.4 Physical characteristics of an axially-stratified threelayers isotropic medium.

Table 4.5 Eigenvalues for an axially-stratified three-layers isotropic medium.

Table 4.6 Physical characteristics of an axially-stratified four-layers isotropic medium.

Table 4.7 Eigenvalues for an axially-stratified four-layers isotropic medium.

Table 4.8 Physical characteristics of an axially-stratified five-layers isotropic medium.

Table 4.9 Eigenvalues for an axially-stratified five-layers isotropic medium.

Table 4.10 Physical characteristics of an axially-stratified five-layers anisotropic medium.

Table 4.11 Eigenvalues for an axially-stratified five-layers anisotropic medium.

Table 4.12 Eigenvalues for an axially-stratified five-layers isotropic medium with $\sigma=10^{-6} \mathrm{~S} / \mathrm{m}$ (TM mode).

Table 4.13 Eigenvalues for an axially-stratified five-layers isotropic medium with $\sigma=10^{-5} \mathrm{~S} / \mathrm{m}$ (TM mode).

Table 4.14 Eigenvalues for an axially-stratified five-layers isotropic medium with $\sigma=10^{-4} \mathrm{~S} / \mathrm{m}$ (TM mode).

Table 4.15 Eigenvalues for an axially-stratified five-layers isotropic medium with $\sigma=10^{-3} \mathrm{~S} / \mathrm{m}$ (TM mode).

Table 4.16 Eigenvalues for an axially-stratified five-layers isotropic medium with $\sigma=10^{-2} \mathrm{~S} / \mathrm{m}$ (TM mode).

Table 4.17 Eigenvalues for an axially-stratified five-layers isotropic medium with $\sigma=10^{-1} \mathrm{~S} / \mathrm{m}$ (TM mode).

Table 4.18 Eigenvalues for an axially-stratified five-layers isotropic medium with $\sigma=1 \mathrm{~S} / \mathrm{m}$ (TM mode). 


\section{List of Abbreviations}

AC Alternating Current

BEM Boundary Element Method

CEM Computational Electromagnetics

EM Electromagnetic

FDTD Finite Difference Method in Time Domain

FEM Finite Element Method

FVM Finite Volume Method

IOUT Internet of Underground Things

LF Low Frequency

LHS The left-hand side of an equation

LWD Logging While Drilling

MMT Mode-Matching Technique

MoM Method of Moment

MWD Measuring While Drilling

PEC Perfect Electric Conductor

RHS The right-hand side of an equation

TCA Tilted-Coil-Antenna

TE Transverse Electric

TI Transverse Isotropic

TM Transverse Magnetic 


\section{Introduction}

\section{1}

\section{General Introduction}

Wireless communications are paramount for today's engineering applications. For practical macroscopic engineering, the electromagnetic (EM) propagation through a given medium is governed by Maxwell's equations, and a physical model can be established on the constitutive parameters permittivity and permeability of the environment. Such constitutive characteristics will determine the behavior of EM waves in terms of its intensity, power flow direction, polarization, dispersion, spacial anisotropic coupling, etc. In this work, our research investigation is on electromagnetic propagation for the oil and gas industry, where complex (anisotropic, inhomogeneous, and dissipative) media are prevalent.

The EM wave propagation in complex geophysical media has been studied for many authors $[2,3,4,5]$ due to its importance to many areas of extractive industry (including mining and the petroleum exploitation) as well as the precise agriculture. The oil industry is one of the most prominent in the demand for novel solutions $[6,7]$. In special, there is a continuous development of sensors for profiling the soil to favor cost-effective oil wells.

EM well-logging tools are one of the many classes of well-logging tools used in oil exploration. It has been subject of interest for many decades due to their capability to measure the conductivity or resistivity of an earth formation [8]. At low operating frequencies (up to $100 \mathrm{MHz}$ ), the electrical resistivity of a soil formation allows us to predict the presence or absence of fossil hydrocarbons. The resistivity profiling is an important ingredient that geophysical scientists employ to make decisions about potential payzones to be explored. In that regard, researchers and the oil industry have been developing advanced logging-while-drilling (LWD) and measurementwhile-drilling (MWD) sensors $[2,8,9]$. These sensors allow real-time data. In order to obtain accurate formation evaluation models, robust Computational Electromagnetics (CEM) methods are necessary for modeling the response of EM tools in complex formations. 
In the last decades, driven by the availability of ever-more-powerful computers, the CEM has experienced a notable flourishment as mature guidance for solving complex real-life electromagnetic problems. The solution, whether computational or analytical, is extremely important for analyzing electromagnetic propagation between wave transmitters and receivers and with their environment [10]. Such analytical or computational solutions are useful in increasing productivity, providing improvement procedures for existing designs, and facilitating the design of new processes and devices.

There are many numerical methods available today for modeling geological formations. These methods are usually based on the spatial discretization of Maxwell's equations on the grounds of the finite-difference time-domain method (FDTD) $[11,12,13]$ and the finite element method (FEM) [14, 15, 16]. Also, other numerical techniques such as the finite volume method (FVM) $[17,18]$, the method of moments (MoM) and the boundary element method (BEM) [19] are also used. Such methods, however, present a high cost in terms of computational time and RAM memory requirements, becoming prohibitive for large problems as well as in iterative solvers.

Many researchers have developed analytical [20], numerical and pseudoanalytical CEM methods $[2,11,21]$ for alleviating the computational cost of large- and multi-scale problems. The research of novel semi-analytical approaches is today essential for conferring robustness and accuracy to representative models where the purely numerical one fails.

The main objective of this dissertation is to explore novel algorithms for modeling electromagnetic wave propagating phenomena in anisotropic media conforming to the cylindrical coordinate system. Our studies dwell robust and numerically efficient computational electromagnetics methods using analytical and semi-analytical techniques. The proposed method allows the analysis of geophysical scenarios analogous to those of the Brazilian Pre-Salt where high conductivity carbonate rocks are predominant. Moreover, the effect of the preand post-salt formations on the electromagnetic fields can be analyzed via a planarly-layered stratum in order to model various representative problems of oil well wireless telemetry systems in anisotropic environments. Yet on the petroleum industry concerns, the methods explored in this study can be employed to the modeling of electromagnetic logging tools. In addition, our mathematical model also has applications for the so-called internet of underground things (IOUT), especially in the area of precision agriculture by using soil characterization via electromagnetic sensors.

The main scientific contribution of this work relies on a novel formulation to analyze the propagation of electromagnetic fields in anisotropic media 


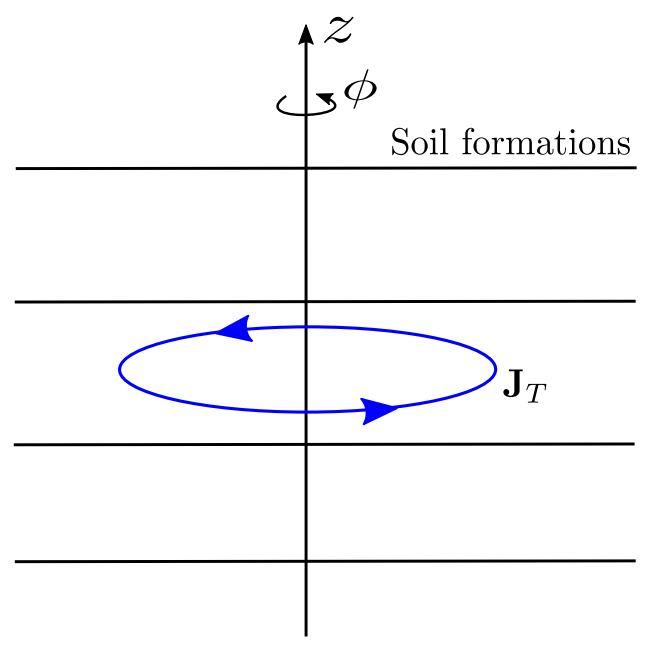

Figure 1.1: Solenoidal source within an axially-stratified geophysical formation.

comprising planar layers as depicted in Fig. 1.1. The fundamental advantage of the methodology developed here is that our approach allows us to compute the field solutions once for any position of source or receptor via a discrete sum of model eigenfield contributions. In this way, we take the edge off calculating a radiation integral each time we change the transmitter and receiver positions as in traditional formalism [2]. Our approach allows a robust and numerically efficient computational method for modeling complex geophysical sensors. The fundamental application of this accurate, fast, and stable semi-analytical solution is the analysis of low-frequency electromagnetic wave propagation for wireless telemetry systems.

\section{2}

\section{Organization of the Dissertation}

This dissertation is divided into six chapters and is organized as follows. In Chapter 2, we presented an overview of the standard electromagnetic propagation modeling for subsoil environments. Some computational electromagnetics methods are also presented as well as their relations with analytical and semi-analytical methods.

In Chapter 3, we introduce a mathematical formulation from Maxwell's equations for describing electric and magnetic fields in both axial and transversal direction to propagation for decoupled TE and TM counterparts.

In Chapter 4, we described the boundary-value problem for representing an axially-stratified soil formation. In this sense, the derive generalized Fresnel coefficients for accounting multiple reflections and transmissions in complex anisotropic media. Such findings represent a generalization of the isotropic model in [2]. Our mathematical formulations as the advantage of employing 
stable scattering-based scalar coefficients (instead of matrix-based ones) for relating the field amplitudes generated at the of a source with the other where the fields will de observed. Some simulation results are presented in order to verify the accuracy and efficiency of the proposed technique.

In Chapter 5, we present a novel and detailed mathematical formulation for expanding the fields due to a solenoidal source in terms of the solution eigenfields obtained in Chapter 3. Simulation results are provided in order to evaluate the performance of the proposed method.

Finally, Chapter 6 is dedicated to presenting the final conclusions of this study. We summarize the main results as well as we suggest some further directions for further researches. 


\section{2 \\ Electromagnetic Propagation Overview for Oil Well}

\section{1}

\section{Introduction}

An oil well is considered a hole dug into the Earth that is designed with the purpose of bringing petroleum oil or other hydrocarbons, such as natural gas, to the surface. Well-logging is an essential field in petrophysical exploration. Its objective is to locate and extract oil and gas in a formation along a borehole using physical measurements [15].

This chapter presents an overview of the mechanisms for electromagnetic propagation. Also, the underground media will be described in terms of its physical and electromagnetic properties. A brief description of the electromagnetic logging tools and some of the usual analytical and computational electromagnetic methods used in wireless telemetry systems in geophysical explorations will be revised.

\section{2 \\ Underground Media Characterization}

The knowledge of the underground media is vital for oil location and extraction in geophysical exploration. The electromagnetic well-logging tool response is directly correlated with the soil formation and the geometry where such sensor is immersed. Formations are typically classified as homogeneous, cylindrically-layered, vertically-layered, as well as isotropic, transverse isotropic (TI), and biaxial anisotropic, etc. The formation itself can exhibit inhomogeneities such as layered beds, dipping beds, anisotropic response, and invaded zones [15].

Electromagnetic characterization of the underground media is required to analyze the EM propagation. Low-frequencies are mostly employed because of the high attenuation the fields suffer over $100 \mathrm{MHz}$. In the view of the research and improvement of mathematical models for describing the electromagnetic propagation, it is necessary first to understand more deeply the electrical properties of the soil and the rocks typically found in geophysical scenarios. 


\subsection{1}

\section{Physics Fundamentals}

Rocks are minerals aggregates with a certain degree of cohesion or consolidation that compose the solid masses of the earth's crust and mantle. They are geologically classified according to the physical process responsible by the origin of their formations as magmatic or igneous, sedimentary, and metamorphic. Magmatic or igneous rocks come from the consolidation of magma, which is a material originated inside the Earth. Sedimentary rocks are derived from other rocks, mainly from the segregation of igneous rocks by a series of physical-chemical processes. Metamorphic rocks are strongly anisotropic media and can be generated from variations of the pressure and temperature conditions of other rock types [22].

The underground soil can be considered as porous media or heterogeneous media. Porous media are mainly characterized by their intrinsic properties of permeability, porosity and electrical formation factor. Although rocks are inherently heterogeneous materials, their precise and detailed knowledge is almost impossible because they occur on multiple scales, which makes them have a stochastic character [15].

The physical, chemical and geometric properties of rocks depend on the physical, chemical and geometric properties of individual minerals, their volume fractions and their distribution. The micro-structure of the rocks exhibits a wide variety of heterogeneities like variability in mineralogy and number and size of pores [22]. In addition, rocks have a wide range of properties where the particular analysis will depend on the application. For this case, the electrical conductivity will be the fundamental observed property.

\subsection{2}

\section{Electromagnetic Characterization}

Electromagnetic measurement techniques are routinely used to probe the Earth at more than $10^{6} \mathrm{MHz}$ for deep magnetic probing, and up to microwave frequencies $\left(10^{9}--10^{11} \mathrm{~Hz}\right)$. At high frequencies, microwave radiometry visualizes the structure in Earth's layered over distances of a fraction of a meter. On the other hand, at lower frequencies, the deep magnetic probe can penetrate more than one hundred kilometers. At very high frequencies, dielectric properties prevail, while low frequencies provide us electrical conductivity information [22].

The understanding of the electromagnetic response of rocks in terms of their electrical conductivity requires knowledge of the electrical properties of the rocks. In fact, the electrical properties of rocks are being used routinely 
in applications such as exploration of oil wells, mining, prospecting of mineral deposits and other purposes in geology and geophysics.

Electromagnetic well-logging tools are designed to measure either the resistivity or the dielectric constant of downhole formations. From the results of these measurements, it was possible to obtain that solid materials exhibit a very large conductivity range. Thus, rocks and rock-forming minerals are also quite varied in their electrical properties with conductivities ranging from $10^{4}$ to $10^{-14} \mathrm{~S} / \mathrm{m}$ [22]. The measured conductivity represents an average effective conductivity of the earth formation in the vicinity of the EM tool. The effective constant or conductivity of the rock is a function of the dielectric constant or conductivity of each component, the microgeometry of the rock frame, and the volume fractions of the components. The components in the pore space can be oil, water, and gas and the resistivity of the rocks is directly related to the properties of these materials and geometry of the rock pores [15].

\section{3}

\section{Electromagnetic Logging Tools and Techniques}

Today's oil and gas exploration requires sophisticated processes to be a profitable business. Once the oil reservoir is located, pilot drilling will be done. The geophysical properties of the earth formations will be studied based on drilling samples and logging data. Logging can be done during drilling or after drilling. When the measurement equipment is directly attached to a drilling bit, the measurement is performed during the drilling, using the LWD or MWD tools [23]. These techniques send the information to a telemetry system, which communicates with the surface platform in real-time [15]. This approach has the advantage to allows to petrophysicists to make decisions in real-time, reducing the operational costs, and optimizing the drilling operation. For these reasons, they become one of the most widely used techniques today and several works $[2,4,17,24,25,26,27,28,29,30]$ have studied methods for its improvement.

In [30] is presented a low-cost computational method for the analysis of the time-domain response of LWD sensors in complex soil formations. The mode matching technique (MMT) [28, 30] is used in [25] as an efficient method to analyze the electromagnetic propagation in a model circular structures filled with complex materials, where it considers an uniaxially anisotropy to describes each layer of a stratified cylindrical waveguide. Also considering a uniaxial anisotropy, a perturbation method with a quite fast computational time is presented in [29] to model electromagnetic fields that describe LWD tools. 
The operating mechanism of electromagnetic sensing tools can be explained in a simplified form as an array of solenoidal antennas properly spaced along the bit of the drilling mandrel. The transmitter antenna is excited by an alternating current $(\mathrm{AC})$. This current generates an EM field that propagates through the formation by inducing an electric current on the receiver antennas. Thus, the received current or voltage is directly proportional to the conductivity of the formation surrounding the oil well. This process allows us to estimate the resistivity of the earth formation [11].

The transmitters and receivers are generally reported in the literature as antennas in the shape of coils and solenoids. The most used antennas are wrapped around the mandrel of the well along the longitudinal axis of the tool. They radiate transversal electric (TE) fields (to the longitudinal directions) due to their spatial orientation. Another class of popular antenna used in such sensors is the tilted-coil-antenna (TCA), which allows the azimuth sensibility for MWD and LWD sensors because the radiation of both TE and transversal magnetic (TM) fields are allowed. All these antennas are placed in different ways on the tool providing diverse readings of characteristics of the formation around the well [31]. In [32] and [1,33], the response of logging tools employing tilted-coil antennas in isotropic and anisotropic medium are characterized.

EM logging tools can reveal relevant characteristics for the oil profiling process. They can operate thousands feet underneath the earth surface in a complex environment. Depending on the relative conductivity of the formation that involves the drilling region, more complex studies of the formation can be performed. These include, for example, studies on the heterogeneity and anisotropy of a given medium. Such tools can provide a range of features that approximate real cases found by the oil industry in an exploration scenario. Moreover, the EM logging tools are widely used in the geophysical formation evaluation and geosteering activities $[15,31]$.

The performance of a logging tool is related to the tool structure, which can vary the complexity, depending on the purpose of the analysis. Well logging tools map formations locally on a very small scale. Therefore, well logging methods have relatively high spatial resolution [15]. Consequently, the logging data gives a more detailed geophysical description to petrophysicists about the formation surrounding the well drilled.

Before computational advances, experiments that required large costs and approximate analytical techniques were the main methods to analyze the response of the EM logging tools [34, 35]. With the advance of computational power, it was possible to model computationally these tools in more complex environments in a more efficient way. 


\section{4 \\ Computational Electromagnetics}

Techniques for solving classical electromagnetism problems have evolved over time. In the beginning, the analysis and design of electromagnetic devices and structures were done experimentally, achieving their characterization. This result was used to the development of new technologies. Subsequently, purely analytical models emerged with solutions obtained in closed form. These solutions model and describe the electromagnetic phenomenon through a simplified algebraic equation under ideal situations [36]. Therefore, when the geometry that defines the problem is simple, finding analytical solutions is a relatively easy task, otherwise it is a bit complicated.

With the advent of high capacity computers, numerical methods have become a fundamental tool for solving electromagnetism problems. These methods can not be applied to all cases. Each problem must be studied until to find one or more formulations whose results are compatible with the analytical solution if it is known, or validated by measurements.

Currently, the numerical solution is achieved using computational algorithms, which handle various numerical analysis techniques. These techniques usually discretize the Maxwells's equations in time and space [36]. Many numerical analysis techniques have been developed in recent years, leading to advances in this area referred to as CEM, one of the most important areas of engineering today. The wide range of electromagnetic problems has led to the development of different algorithms in CEM with its advantages and limitations [37]. Low-frequency (LF) algorithms or numerical methods have become more available in analyzing logging tools.

The FDTD method $[38,39,40,41]$ excels at study of wave propagation in complex materials and at analysis of inhomogeneous and nonlinear media, though its demands for system memory are high due to discretization of the entire solution domain, and it suffers from dispersion issues and the need to artificially truncate of the solution boundary [37]. This numerical technique was used in [42] to simulate the response of LWD tools through anisotropic conductive formations in cylindrical coordinates. This method is also addressed in [43] to compute the electromagnetic field due to electrical and magnetic dipoles embedded in a layered medium.

The FEM $[44,45]$ is a method used to solve frequency-domain boundaryvalued electromagnetic problems via variational techniques. As in the FDTD, the solution domain must be discretized and truncated, making the FEM approach often unsuitable for radiation or scattering problems unless combined with a boundary integral equation approach [46]. This method is presented 
in [47] to simulate the response of a electromagnetic LWD tool in a three dimensional (3D) inhomogeneous and horizontal layered medium.

The MoM is widely used for solving radiation and scattering problems through electromagnetic surface or volume integral equations in the frequency domain. This method differs from FDTD and FEM as the electromagnetic sources are the quantities of interest, and so only the surface or volume of the antenna or scatterer must be discretized [37]. This method is used in [48] to analyze circularly symmetric reflectors in a simple analytical form to reduce computational requirements as much as possible.

The above-mentioned methods are typically limited to problems of small electrical size due to limitations of computation time and the system memory. Indeed, low-frequency methods are so-named because they solve Maxwell's equations with no implicit approximations, and are more demanding in terms of CPU [37]. Thus, although these methods can be applied with precision, the speed of simulation restricts their application due to the high computational consumption that they require. This disadvantage is even more so in situations where the set of coupled layers compose complex geophysical formations. Several pseudo-analytical algorithm and techniques to model electromagnetic telemetry and determine the fields components along planar stratified media have been proposed in literature [11, 29, 33, 49, 50]. A pseudo-analytical method is presented in [2, Ch. 2] for modeling line and point sources in planarly-layered isotropic media in terms of Fourier-type radiation integrals. The authors in $[1,3,51]$ presented numerical matching methods (NMM) to model electromagnetic telemetry systems of vertical and directional wells in both radial and axial stratifications. These methods were implemented in order to mitigate the disadvantage of the computational demand of purely numerical brute-force methods. In what follows, we will employ the key idea of such well-known semi-analytic CEM to study novel and more robust approaches to dealing with stratified anisotropic media for underground engineering applications. 


\section{3}

\section{Electromagnetic Field Theory}

\section{1}

\section{Introduction}

A mathematical formulation from the Maxwell's equations will be introduced in this chapter to derive the electric and magnetic fields. This fields will be obtained for decoupled TE and TM modes in both axial and transversal components to axial propagation direction. The study will be done in a uniaxially anisotropic and axially-stratified medium, where the inclusion of anisotropy makes the mathematical analysis quite complicated, but very useful for any geophysical problem. The final expressions of fields will be ready to apply the boundary conditions in the next chapter, which are imposed by the geometry of our problem.

\section{2}

\section{Maxwell's equations}

James Clerk Maxwell's equations represent, under a mathematical model, the laws of electromagnetic phenomena. These equations determine the relationships between the electric flux density (D), the magnetic flux density (B), the electric current density $(\mathbf{J})$ and the magnetic current density $(\mathbf{M})$. Such relationships are a function of variation of the electric field intensity $(\mathbf{E})$ and the magnetic field intensity $(\mathbf{H})$ in space and time. This is true for both fields in space and material substances [52].

The notation adopted in this work is similar to that used in [2] and [53]. Assuming and omitting a time-harmonic dependence in the form $\exp (-i \omega t)$, the Maxwell's equations in the differential form in a homogeneous anisotropic medium are

$$
\begin{aligned}
\boldsymbol{\nabla} \times \mathbf{E} & =i \omega \overline{\bar{\mu}} \cdot \mathbf{H}, \\
\boldsymbol{\nabla} \times \mathbf{H} & =-i \omega \overline{\bar{\epsilon}} \cdot \mathbf{E}+\mathbf{J}, \\
\boldsymbol{\nabla} \cdot \mathbf{D} & =\varrho_{e}, \\
\boldsymbol{\nabla} \cdot \mathbf{B} & =0,
\end{aligned}
$$




$$
\begin{aligned}
& \mathbf{D}=\overline{\bar{\epsilon}} \cdot \mathbf{E} \\
& \mathbf{B}=\overline{\bar{\mu}} \cdot \mathbf{H}
\end{aligned}
$$

where $\mathbf{E}$ is given in $\mathrm{V} / \mathrm{m}, \mathbf{J}$ in $\mathrm{A} / \mathrm{m}^{2}, \mathbf{H}$ in $\mathrm{A} / \mathrm{m}$ and $\varrho_{e}$ is the electric charge density in $\mathrm{C} / \mathrm{m}^{3}$. The media is characterized by the complex magnetic permeability tensor

$$
\overline{\bar{\mu}}=\left[\begin{array}{ccc}
\mu_{s} & 0 & 0 \\
0 & \mu_{s} & 0 \\
0 & 0 & \mu_{z}
\end{array}\right]=\operatorname{diag}\left(\mu_{s}, \mu_{s}, \mu_{z}\right), \quad \text { with } \quad \mu_{\{s, z\}}=\mu_{0} \mu_{r\{s, z\}}
$$

and by the complex electric permittivity tensor

$$
\overline{\bar{\epsilon}}=\left[\begin{array}{ccc}
\epsilon_{s} & 0 & 0 \\
0 & \epsilon_{s} & 0 \\
0 & 0 & \epsilon_{z}
\end{array}\right]=\operatorname{diag}\left(\epsilon_{s}, \epsilon_{s}, \epsilon_{z}\right), \quad \text { with } \quad \epsilon_{\{s, z\}}=\epsilon_{0} \epsilon_{r\{s, z\}}+i \sigma_{\{s, z\}} / \omega,
$$

both represented in cylindrical coordinates, where the subscript $s=\{\rho, \phi\}$. The terms $\mu_{0}=4 \pi \times 10^{-7} \mathrm{H} / \mathrm{m}$ and $\epsilon_{0}=8.854 \times 10^{-12} \mathrm{~F} / \mathrm{m}$ are the values of permeability and permittivity in vacuum. The relationship between these two values is given by the propagation velocity of an electromagnetic wave in vacuum. The terms $\mu_{r\{s, z\}}, \epsilon_{r\{s, z\}}$ and $\sigma_{\{s, z\}}$ are the relative magnetic and dielectric constants and the effective electrical conductivity of the medium, respectively.

Permeability, permittivity and conductivity are the EM parameters that define a medium where an EM wave was propagated. The effective dielectric constant and conductivity are interrelated. For high frequencies, the displacement phenomena dominate over the conductivity phenomena. In this way, the media are usually characterized from their dielectric constant. For low frequencies, the term of conductivity predominates.

\section{3}

\section{Source-Free Axial Electromagnetic Fields}

In a source-free region and decomposing the fields in axial (along $\hat{z}$ ) and transverse ( $\hat{s}$ direction) components to $\hat{z}$, we have that

$$
\mathbf{F}=\mathbf{F}_{s}+\hat{z} F_{z}
$$

in which $\mathbf{F}=\{\mathbf{E}, \mathbf{H}\}, \mathbf{F}_{s}=\left\{\mathbf{E}_{s}, \mathbf{H}_{s}\right\}$ and $F_{z}=\left\{E_{z}, H_{z}\right\}$, and

$$
\boldsymbol{\nabla}=\boldsymbol{\nabla}_{s}+\hat{z} \frac{\partial}{\partial z}
$$

where $\nabla_{s}$ denotes the transverse two-dimensional nabla operator given by 


$$
\nabla_{s}=\hat{\rho} \frac{\partial}{\partial \rho}+\hat{\phi} \frac{1}{\rho} \frac{\partial}{\partial \phi}
$$

Using the procedure described in [2] and [1], taking the curl of the equation $(3-1)$ and projecting the resulting vector onto $\hat{z}$, we obtain

$$
\hat{z} \cdot[\nabla \times(\boldsymbol{\nabla} \times \mathbf{E})]=i \omega \hat{z} \cdot[\nabla \times(\overline{\bar{\mu}} \cdot \mathbf{H})],
$$

working in the right-hand side (RHS) of the previous equation,

$$
\begin{aligned}
& i \omega \hat{z} \cdot[\boldsymbol{\nabla} \times(\overline{\bar{\mu}} \cdot \mathbf{H})]=i \omega \hat{z} \cdot\left\{\left(\boldsymbol{\nabla}_{s}+\hat{z} \frac{\partial}{\partial z}\right) \times\left[\left(\overline{\bar{\mu}}_{s}+\hat{z} \hat{z} \mu_{z}\right) \cdot\left(\mathbf{H}_{s}+\hat{z} H_{z}\right)\right]\right\} \\
&=i \omega \hat{z} \cdot\left[\boldsymbol{\nabla}_{s} \times\left(\overline{\bar{\mu}_{s}} \cdot \mathbf{H}_{s}\right)+\boldsymbol{\nabla}_{s} \times\left(\hat{z} \mu_{z} H_{z}\right)+\frac{\partial}{\partial z} \hat{z} \times\left(\overline{\bar{\mu}}_{s} \cdot \mathbf{H}_{s}\right)+\right. \\
&\left.\frac{\partial}{\partial z} \hat{z} \times \hat{z} \mu_{z} H_{z}\right] \\
&= i \omega \hat{z} \cdot\left[\nabla_{s} \times\left(\mu_{s} \overline{\bar{I}} \cdot \mathbf{H}_{s}\right)\right] \\
&= i \omega \mu_{s} \hat{z} \cdot\left(\nabla_{s} \times \mathbf{H}_{s}\right) .
\end{aligned}
$$

From equation (3-2) we have that

$$
\left(\boldsymbol{\nabla}_{s}+\hat{z} \frac{\partial}{\partial z}\right) \times\left(\mathbf{H}_{s}+\hat{z} H_{z}\right)=-i \omega\left(\overline{\overline{\epsilon_{s}}}+\hat{z} \hat{z} \epsilon_{z}\right) \cdot \mathbf{E},
$$

separating in components $\hat{s}$ and $\hat{z}$,

$$
\left\{\begin{array}{ccc}
\hat{s}: & \nabla_{s} \times \hat{z} H_{z}+\frac{\partial}{\partial z} \hat{z} \times \mathbf{H}_{s} & =-i \omega \overline{\bar{\epsilon}_{s}} \cdot \mathbf{E}_{s} \\
\hat{z}: & \boldsymbol{\nabla}_{s} \times \mathbf{H}_{s} & =-i \omega \hat{z} \epsilon_{z} E_{z},
\end{array}\right.
$$

projecting the component $\hat{z}$ of the equation (3-13) onto $\hat{z}$ and substituting in the equation (3-12), we can find

$$
\hat{z} \cdot[\nabla \times(\nabla \times \mathbf{E})]=\omega^{2} \mu_{s} \epsilon_{z} E_{z} .
$$

From the Gauss's law for the magnetism, equation (3-4), we obtain

$$
\begin{aligned}
\boldsymbol{\nabla} \cdot(\overline{\bar{\mu}} \cdot \mathbf{H}) & =\left(\boldsymbol{\nabla}_{s}+\hat{z} \frac{\partial}{\partial z}\right) \cdot\left(\mu_{s} \mathbf{H}_{s}+\hat{z} \mu_{z} H_{z}\right) \\
& =\mu_{s} \boldsymbol{\nabla}_{s} \cdot \mathbf{H}_{s}+\mu_{z} \frac{\partial}{\partial z} H_{z} \\
& =\mu_{s} \boldsymbol{\nabla} \cdot \mathbf{H}-\mu_{s}\left(1-\frac{\mu_{z}}{\mu_{s}}\right) \frac{\partial H_{z}}{\partial z}=0
\end{aligned}
$$


then,

$$
\boldsymbol{\nabla} \cdot \mathbf{H}=\left(1-\frac{\mu_{z}}{\mu_{s}}\right) \frac{\partial H_{z}}{\partial z} .
$$

A dual equation can be easily established for the electric field as

$$
\boldsymbol{\nabla} \cdot \mathbf{E}=\left(1-\frac{\epsilon_{z}}{\epsilon_{s}}\right) \frac{\partial E_{z}}{\partial z}
$$

The equations (3-15) and (3-16) show that the divergence of the electric and magnetic fields do not vanish anymore as in the isotropic media for the limits $\mu_{s}=\mu_{z}$ and $\epsilon_{s}=\epsilon_{z}[2,54]$.

Using the vector identity [55]

$$
\boldsymbol{\nabla} \times \boldsymbol{\nabla} \times \mathbf{F}=\boldsymbol{\nabla}(\boldsymbol{\nabla} \cdot \mathbf{F})-(\boldsymbol{\nabla} \cdot \boldsymbol{\nabla}) \mathbf{F}
$$

and projecting it onto $\hat{z}$ for to find the left-hand side (LHS) of the equation (3-14), we obtain

$$
\begin{aligned}
\hat{z} \cdot[\boldsymbol{\nabla} \times(\boldsymbol{\nabla} \times \mathbf{E})] & =\hat{z} \cdot \boldsymbol{\nabla}(\boldsymbol{\nabla} \cdot \mathbf{E})-\hat{z} \cdot(\boldsymbol{\nabla} \cdot \boldsymbol{\nabla}) \mathbf{E} \\
& =\hat{z} \cdot\left(\boldsymbol{\nabla}_{s}+\hat{z} \frac{\partial}{\partial z}\right)\left(1-\frac{\epsilon_{z}}{\epsilon_{s}}\right) \frac{\partial E_{z}}{\partial z}-\hat{z} \cdot \nabla^{2} \mathbf{E} \\
& =\left(1-\frac{\epsilon_{z}}{\epsilon_{s}}\right) \frac{\partial^{2} E_{z}}{\partial z^{2}}-\nabla^{2} E_{z},
\end{aligned}
$$

where $\nabla^{2}$ is the scalar Laplacian in cylindrical coordinates defined as [55]

$$
\nabla^{2} f=\frac{1}{\rho} \frac{\partial}{\partial \rho}\left(\rho \frac{\partial f}{\partial \rho}\right)+\frac{1}{\rho^{2}} \frac{\partial^{2} f}{\partial \phi^{2}}+\frac{\partial^{2} f}{\partial z^{2}}
$$

After substituting (3-18) into (3-14), we can find

$$
\nabla_{s}^{2} E_{z}+\frac{\epsilon_{z}}{\epsilon_{s}} \frac{\partial^{2} E_{z}}{\partial z^{2}}+\omega^{2} \mu_{s} \epsilon_{z} E_{z}=0
$$

in which the two-dimensional Laplacian operator is given by

$$
\begin{aligned}
\nabla_{s}^{2} f & =\nabla^{2} f-\frac{\partial^{2} f}{\partial z^{2}} \\
& =\frac{1}{\rho} \frac{\partial}{\partial \rho}\left(\rho \frac{\partial f}{\partial \rho}\right)+\frac{1}{\rho^{2}} \frac{\partial^{2} f}{\partial \phi^{2}}
\end{aligned}
$$

Similarly, a wave equation dual to (3-20) can be found for the axial magnetic 
field using a procedure analogous to that shown above. It follows that

$$
\nabla_{s}^{2} H_{z}+\frac{\mu_{z}}{\mu_{s}} \frac{\partial^{2} H_{z}}{\partial z^{2}}+\omega^{2} \mu_{z} \epsilon_{s} H_{z}=0
$$

The equations (3-20) and (3-23) are the homogeneous Helmholtz wave equations for $E_{z}$ and $H_{z}$, respectively. In order to solve these equations in cylindrical coordinates, the method of separation of variables can be used [55]. The scalar Helmholtz equation for axial fields in cylindrical coordinates is defined by

$$
\left[\frac{1}{\rho} \frac{\partial}{\partial \rho}\left(\rho \frac{\partial}{\partial \rho}\right)+\frac{1}{\rho^{2}} \frac{\partial^{2}}{\partial \phi^{2}}+\frac{p_{z}}{p_{s}} \frac{\partial^{2}}{\partial z^{2}}+\omega^{2} p_{z} \tilde{p_{s}}\right] F_{z}=0,
$$

where $p=\{\epsilon, \mu\}$ and $\tilde{p}=\{\mu, \epsilon\}$. Following the method of separation of variables, we find the elementary solution to the equation (3-24) in the form

$$
F_{z}=R(\rho) \Phi(\phi) Z(z)
$$

After substituting the equation (3-25) into (3-24), dividing by $F_{z}$ and considering that the partial derivatives becomes total ones, can be derived

$$
\frac{1}{\rho R} \frac{d}{d \rho}\left(\rho \frac{d R}{d \rho}\right)+\frac{1}{\rho^{2} \Phi} \frac{d^{2} \Phi}{d \phi^{2}}+\frac{p_{z}}{p_{s}} \frac{1}{Z} \frac{d^{2} Z}{d z^{2}}+\omega^{2} p_{z} \tilde{p}_{s}=0 .
$$

By noting that the third term is independent of $\rho$ and $\phi$, it must be also independent of $z$ because the equation is to sum zero for all $\rho, \phi, z$. Hence, we can do

$$
\frac{1}{Z} \frac{d^{2} Z}{d z^{2}}=-k_{z}^{2}
$$

where $k_{z}$ is a constant and the minus sign is chosen for convenience in the resulting solutions. Substituting (3-27) into (3-26) and multiplying by $\rho^{2}$, we find

$$
\frac{\rho}{R} \frac{d}{d \rho}\left(\rho \frac{d R}{d \rho}\right)+\frac{1}{\Phi} \frac{d^{2} \Phi}{d \phi^{2}}+\left(\omega^{2} p_{z} \tilde{p}_{s}-\frac{p_{z}}{p_{s}} k_{z}^{2}\right) \rho^{2}=0
$$

The second term of the equation (3-28) is independent of $\rho$ and $z$, as well as the other terms are independents of $\phi$. Hence, we can assume that

$$
\frac{1}{\Phi} \frac{d^{2} \Phi}{d \phi^{2}}=-n^{2}
$$

where $n$ is a constant. After substitution the above into (3-28), we obtain

$$
\frac{\rho}{R} \frac{d}{d \rho}\left(\rho \frac{d R}{d \rho}\right)-n^{2}+\left(\omega^{2} p_{z} \tilde{p}_{s}-\frac{p_{z}}{p_{s}} k_{z}^{2}\right) \rho^{2}=0 .
$$

This equation above describes an equation that depends on $\rho$ only. Hence, in order to summarize, we can introduce

$$
k_{\rho}^{2}=\frac{p_{z}}{p_{s}}\left(k_{s}^{2}-k_{z}^{2}\right)
$$


and the wavenumber related to the transversal anisotropy given as

$$
k_{s}^{2}=\omega^{2} p_{s} \tilde{p}_{s}=\omega^{2} \epsilon_{s} \mu_{s}
$$

Then, the wave equation can be separated by the equations (3-27), (3-29) and (3-30) such as

$$
\begin{aligned}
\rho \frac{d}{d \rho}\left(\rho \frac{d R}{d \rho}\right)+\left[\left(k_{\rho} \rho\right)^{2}-n^{2}\right] R & =0 \\
\frac{d^{2} \Phi}{d \phi^{2}}+n^{2} \Phi & =0 \\
\frac{d^{2} Z}{d z^{2}}+k_{z}^{2} Z & =0 .
\end{aligned}
$$

The equation (3-33) is a Bessel differential equation of order $n$, whose solutions are in general, a linear superposition of any two of the linearly independent Bessel and Hankel functions of first and second kind, this is, $J_{n}\left(k_{\rho} \rho\right), Y_{n}\left(k_{\rho} \rho\right)$, $H_{n}^{(1)}\left(k_{\rho} \rho\right)$ and $H_{n}^{(2)}\left(k_{\rho} \rho\right)$, respectively. The equations (3-34) and (3-35) are onedimensional non-homogeneous Laplaces's equations, whose solutions are given by harmonic functions. Finally, the elementary solution for (3-24) is given by

$$
F_{z}=R_{n}\left(k_{\rho} \rho\right) \Phi(n \phi) Z\left(k_{z} z\right) .
$$

The quantities $k_{\rho}$ and $k_{z}$ can be interpreted as the radial and axial wavenumbers, respectively, and $n$ is the azimuthal index related to the modal propagation constant that gives the azimuthal dependence of fields.

The linear combination of elementary wave functions in the equation (3-36) is also a solution to the equation (3-24) [1, 31, 55]. More general solutions can be constructed by summing over all possible values of the $k_{z}$ (or $k_{\rho}$ ) eigenvalues and $n$ azimuthal indices. Therefore, the general solution that represents the solution to the modal fields would be

$$
F_{z}=\sum_{n} \sum_{k_{z}} C_{n, k_{z}} R_{n}\left(k_{\rho} \rho\right) \Phi(n \phi) Z\left(k_{z} z\right),
$$

where the constants $C_{n, k_{z}}$ can be evaluated when applying the source boundary condition.

\section{4}

\section{Axial Field Components}

In a compact notation, the equation (3-37) can be represented by

$$
\left[\begin{array}{l}
E_{z} \\
H_{z}
\end{array}\right]=\sum_{n=-\infty}^{\infty} \sum_{p=1}^{\infty}\left[\begin{array}{l}
e_{z, n p}(\rho) \\
h_{z, n p}(\rho)
\end{array}\right]\left[\begin{array}{l}
e_{z, n p}\left(k_{z} z\right) \\
h_{z, n p}\left(k_{z} z\right)
\end{array}\right] e^{i n \phi},
$$


in which $p$ is related to radial propagation constant that gives the radial dependence of the fields. The values of $n$ are positive and negative integer due to the choice of the exponential form $\exp (i n \phi)$ and give the azimuthal dependence of the fields. The radially-dependent functions are given by a linear combination of first-kind Hankel and Bessel function of integer order $n$ such as

$$
\left[\begin{array}{l}
e_{z, n p}(\rho) \\
h_{z, n p}(\rho)
\end{array}\right]=\overline{\bar{H}}_{z n}^{(1)}\left(k_{\rho} \rho\right) \bar{a}+\overline{\bar{J}}_{z n}\left(k_{\rho} \rho\right) \bar{b}
$$

where the $k_{\rho, n \rho}$ radial propagation constant in the $\rho$-direction is given by the equation (3-31). We define $B_{z n}=\left\{H_{n}^{(1)}\right.$ or $\left.J_{n}\right\}$, where the most appropriate choice will depend on the problem boundary conditions. The field amplitudes $\bar{a}$ and $\bar{b}$ are vectors $2 \times 1$ defined by the boundary conditions and are given by

$$
\bar{a}=\left[\begin{array}{l}
a_{n p}^{e} \\
a_{n p}^{h}
\end{array}\right] \quad \text { and } \quad \bar{b}=\left[\begin{array}{l}
b_{n p}^{e} \\
b_{n p}^{h}
\end{array}\right]
$$

The fields contribution into the axial direction in (3-38) can be written as

$$
\begin{aligned}
{\left[\begin{array}{l}
e_{j z, n p}\left(k_{j z} z\right) \\
h_{j z, n p}\left(k_{j z} z\right)
\end{array}\right] } & =\left\{\left[\begin{array}{cc}
e^{ \pm i k_{j z}^{e} z} & 0 \\
0 & e^{ \pm i k_{j z}^{h} z}
\end{array}\right]+\right. \\
& {\left.\left[\begin{array}{cc}
\tilde{R}_{j, j \mp 1}^{T M^{(z)}} & 0 \\
0 & \tilde{R}_{j, j \mp 1}^{T E^{(z)}}
\end{array}\right]\left[\begin{array}{cc}
e^{\mp i k_{j z}^{e}\left(z+2 z^{ \pm}\right)} & 0 \\
0 & e^{\mp i k_{j z}^{h}\left(z+2 z^{ \pm}\right)}
\end{array}\right]\right\}\left[\begin{array}{c}
A_{j}^{ \pm e} \\
A_{j}^{ \pm h}
\end{array}\right] }
\end{aligned}
$$

where

$$
z^{ \pm}= \begin{cases}z^{+} & =z_{j-1} \\ z^{-} & =z_{j}\end{cases}
$$

The $k_{z, n p}$ modal propagation constant in the $z$-direction can be obtained from the equation (3-31) as

$$
k_{z}^{2}=k_{s}^{2}-\frac{p_{s}}{p_{z}} k_{\rho}^{2},
$$

where we have $k_{z}^{h}$ for $p=\mu$ and $k_{z}^{e}$ for $p=\epsilon$. The $\tilde{R}_{j, j \mp 1}$ generalized reflection coefficient due to the axially-stratified media which will be determined in the section 4.2 .

Finally, following the compact shape of the equation (3-38), the axial fields can be written in decoupled TE and TM modes. Considering a source that propagates for $+z$ or $-z$ directions, respectively, we have for the TE mode $\left(E_{z}=0\right)$ the $H_{z}$ field given by

$$
H_{j z}=A_{j}^{ \pm h} \sum_{n=-\infty}^{\infty} \sum_{p=1}^{\infty} B_{z n}\left(k_{\rho} \rho\right) e^{i n \phi}\left[e^{ \pm i k_{j z}^{h} z}+\tilde{R}_{j, j \mp 1}^{T E^{(z)}} e^{\mp i k_{j z}^{h}\left(z+2 z^{ \pm}\right)}\right],
$$

and for the TM mode $\left(H_{z}=0\right)$, we have the $E_{z}$ field given by

$$
E_{j z}=A_{j}^{ \pm} \sum_{n=-\infty}^{\infty} \sum_{p=1}^{\infty} B_{z n}\left(k_{\rho} \rho\right) e^{i n \phi}\left[e^{ \pm i k_{j z}^{e} z}+\tilde{R}_{j, j \mp 1}^{T M^{(z)}} e^{\mp i k_{j z}^{e}\left(z+2 z^{ \pm}\right)}\right] .
$$


where $z^{ \pm}$is given by the equation (3-42). Notice that, in order to simplify the notation, we have omitted the subscript $n p$ in the radial and modal propagation constants.

\section{5}

\section{Transversal Field Components}

Starting from the Maxwell's rotational equations (3-1) and (3-2) in a source-free region, we have that

$$
\begin{aligned}
& \left(\boldsymbol{\nabla}_{s}+\hat{z} \frac{\partial}{\partial z}\right) \times\left(\mathbf{E}_{s}+\hat{z} E_{z}\right)=i \omega\left(\overline{\overline{\mu_{s}}} \cdot \mathbf{H}_{s}+\hat{z} \mu_{z} H_{z}\right), \\
& \left(\boldsymbol{\nabla}_{s}+\hat{z} \frac{\partial}{\partial z}\right) \times\left(\mathbf{H}_{s}+\hat{z} H_{z}\right)=-i \omega\left(\overline{\overline{\epsilon_{s}}} \cdot \mathbf{E}_{s}+\hat{z} \epsilon_{z} E_{z}\right),
\end{aligned}
$$

separating the equations (3-46) and (3-47) in components $\hat{s}$ and $\hat{z}$, respectively, it can be found

$$
\begin{aligned}
& \hat{s}\left\{\begin{array}{l}
\boldsymbol{\nabla}_{s} \times \hat{z} E_{z}+\hat{z} \times \frac{\partial}{\partial z} \mathbf{E}_{s}=i \omega \overline{\bar{\mu}}_{s} \cdot \mathbf{H}_{s} \\
\boldsymbol{\nabla}_{s} \times \hat{z} H_{z}+\hat{z} \times \frac{\partial}{\partial z} \mathbf{H}_{s}=-i \omega \overline{\bar{\epsilon}}_{s} \cdot \mathbf{E}_{s}
\end{array} \quad\right. \text { and } \\
& \hat{z}\left\{\begin{array}{l}
\nabla_{s} \times \mathbf{E}_{s}=i \omega \mu_{z} \hat{z} H_{z} \\
\nabla_{s} \times \mathbf{H}_{s}=-i \omega \epsilon_{z} \hat{z} E_{z} .
\end{array}\right.
\end{aligned}
$$

Knowing that $\partial / \partial z \rightarrow \pm i k_{z}$ para $z \gtrless 0$, multiplying the vector $\hat{z}$ on both sides of the equations from $\hat{s}$ component and using the vector identities,

$$
\begin{aligned}
\hat{z} \times\left(\nabla_{s} \times \hat{z} F_{z}\right) & =\nabla_{s} F_{z} \\
\hat{z} \times\left(\hat{z} \times \mathbf{F}_{s}\right) & =-\mathbf{F}_{s}
\end{aligned}
$$

after of some manipulations, we find for $z>0$ that

$$
\begin{aligned}
& \mathbf{E}_{s}=\frac{1}{i k_{z}}\left(\nabla_{s} E_{z}-i \omega \mu_{s} \hat{z} \times \mathbf{H}_{s}\right) \\
& \mathbf{H}_{s}=\frac{1}{i k_{z}}\left(\boldsymbol{\nabla}_{s} H_{z}+i \omega \epsilon_{s} \hat{z} \times \mathbf{E}_{s}\right)
\end{aligned}
$$

Substituting (3-51) into (3-50) and vice-versa or by duality, we have that the 
transversal fields can be expressed as a combination of the axial ones, such as

$$
\begin{array}{r}
\mathbf{E}_{s}=\frac{1}{k_{\rho_{T}}^{2}}\left(i k_{z} \nabla_{s} E_{z}+i \omega \mu_{s} \boldsymbol{\nabla}_{s} \times \hat{z} H_{z}\right) \\
\mathbf{H}_{s}=\frac{1}{k_{\rho_{T}}^{2}}\left(i k_{z} \boldsymbol{\nabla}_{s} H_{z}-i \omega \epsilon_{s} \boldsymbol{\nabla}_{s} \times \hat{z} E_{z}\right),
\end{array}
$$

where $k_{\rho_{T}}^{2}=k_{s}^{2}-k_{z}^{2}$. Substituting (3-11) in both equations above, we have

$$
\begin{aligned}
& \mathbf{E}_{s}=\frac{1}{k_{\rho_{T}}^{2}}\left[\left(i k_{z} \frac{\partial E_{z}}{\partial \rho}+i \omega \mu_{s} \frac{1}{\rho} \frac{\partial H_{z}}{\partial \phi}\right) \hat{\rho}+\left(i k_{z} \frac{1}{\rho} \frac{\partial E_{z}}{\partial \phi}-i \omega \mu_{s} \frac{\partial H_{z}}{\partial \rho}\right) \hat{\phi}\right] \\
& \mathbf{H}_{s}=\frac{1}{k_{\rho_{T}}^{2}}\left[\left(i k_{z} \frac{\partial H_{z}}{\partial \rho}-i \omega \epsilon_{s} \frac{1}{\rho} \frac{\partial E_{z}}{\partial \phi}\right) \hat{\rho}+\left(i k_{z} \frac{1}{\rho} \frac{\partial H_{z}}{\partial \phi}+i \omega \epsilon_{s} \frac{\partial E_{z}}{\partial \rho}\right) \hat{\phi}\right]
\end{aligned}
$$

In a compact shape, the transversal fields can be written as

$$
\left[\begin{array}{c}
\mathbf{E}_{s} \\
\mathbf{H}_{s}
\end{array}\right]=\sum_{n=-\infty}^{\infty} \sum_{p=1}^{\infty}\left[\begin{array}{l}
\mathbf{e}_{s, n p}(\rho) \\
\mathbf{h}_{s, n p}(\rho)
\end{array}\right] e^{i\left(n \phi+k_{z, n p} z\right)}
$$

in which the $\rho$ - and $\phi$-dependent fields can be expressed, respectively, as a linear combination of first-kind Hankel and Bessel function given by

$$
\begin{gathered}
{\left[\begin{array}{l}
e_{\rho, n p}(\rho) \\
h_{\rho, n p}(\rho)
\end{array}\right]=\overline{\bar{H}}_{\rho n}^{(1)}\left(k_{\rho} \rho\right) \bar{a}+\overline{\bar{J}}_{\rho n}\left(k_{\rho} \rho\right) \bar{b}} \\
{\left[\begin{array}{l}
e_{\phi, n p}(\rho) \\
h_{\phi, n p}(\rho)
\end{array}\right]=\overline{\bar{H}}_{\phi n}^{(1)}\left(k_{\rho} \rho\right) \bar{a}+\overline{\bar{J}}_{\phi n}\left(k_{\rho} \rho\right) \bar{b},}
\end{gathered}
$$

where knowing that $\partial / \partial \phi=i n$ and substituting the axial fields $E_{z}$ and $H_{z}$ in the equations (3-54) and (3-55), we have that

$$
\begin{aligned}
& \overline{\bar{B}}_{\rho n}\left(k_{\rho} \rho\right)=\frac{1}{k_{\rho_{T}}^{2} \rho}\left[\begin{array}{cc}
i k_{z}^{e} k_{\rho} \rho B_{n}^{\prime}\left(k_{\rho} \rho\right) & -n \omega \mu_{s} B_{n}\left(k_{\rho} \rho\right) \\
n \omega \epsilon_{s} B_{n}\left(k_{\rho} \rho\right) & i k_{z}^{h} k_{\rho} \rho B_{n}^{\prime}\left(k_{\rho} \rho\right)
\end{array}\right] \\
& \overline{\bar{B}}_{\phi n}\left(k_{\rho} \rho\right)=\frac{1}{k_{\rho_{T}}^{2} \rho}\left[\begin{array}{cc}
-n k_{z}^{e} B_{n}\left(k_{\rho} \rho\right) & -i \omega \mu_{s} k_{\rho} \rho B_{n}^{\prime}\left(k_{\rho} \rho\right) \\
i \omega \epsilon_{s} k_{\rho} \rho B_{n}^{\prime}\left(k_{\rho} \rho\right) & -n k_{z}^{h} B_{n}\left(k_{\rho} \rho\right)
\end{array}\right] .
\end{aligned}
$$

The derivative $B_{n}^{\prime}=\left\{H_{n}^{\prime(1)}\right.$ or $\left.J_{n}^{\prime}\right\}$ is taken with respect to the argument of the function. Elements 11 and 12 of the matrices in the above equations correspond to constants in the electric field. Elements 21 and 22 correspond to constants in the magnetic field. The fields components in (3-59) and (3-60) reduce to isotropic solution found in [2] for $p_{z}=p_{s}$ in the equation (3-31), where these terms were introduced.

Finally, following the compact notation of the equation (3-56), the transversal fields to $\hat{z}$ domain can be expressed in decoupled TE and TM 
modes. Considering a source that propagates to $+z$ or $-z$ directions, respectively, we have for the TE mode

$$
\begin{aligned}
& E_{j \rho}=-\frac{\omega \mu_{j s} A_{j}^{ \pm h}}{k_{\rho_{T}}^{2} \rho} \sum_{n=-\infty}^{\infty} \sum_{p=1}^{\infty} n B_{n}\left(k_{\rho} \rho\right) e^{i n \phi}\left[e^{ \pm i k_{j z}^{h} z}+\tilde{R}_{j, j \mp 1}^{T E^{(z)}} e^{\mp i k_{j z}^{h}\left(z+2 z^{ \pm}\right)}\right] \\
& E_{j \phi}=-\frac{i \omega \mu_{j s} k_{\rho} A_{j}^{ \pm h}}{k_{\rho_{T}}^{2}} \sum_{n=-\infty}^{\infty} \sum_{p=1}^{\infty} B_{n}^{\prime}\left(k_{\rho} \rho\right) e^{i n \phi}\left[e^{ \pm i k_{j z}^{h} z}+\tilde{R}_{j, j \mp 1}^{T E^{(z)}} e^{\mp i k_{j z}^{h}\left(z+2 z^{ \pm}\right)}\right] \\
& H_{j \rho}=\frac{i k_{\rho} A_{j}^{ \pm}}{k_{\rho_{T}}^{2}} \sum_{n=-\infty}^{\infty} \sum_{p=1}^{\infty} k_{j z}^{h} B_{n}^{\prime}\left(k_{\rho} \rho\right) e^{i n \phi}\left[e^{ \pm i k_{j z}^{h} z}-\tilde{R}_{j, j \mp 1}^{T E^{(z)}} e^{\mp i k_{j z}^{h}\left(z+2 z^{ \pm}\right)}\right] \\
& H_{j \phi}=-\frac{A_{j}^{ \pm}}{k_{\rho_{T}}^{2} \rho} \sum_{n=-\infty}^{\infty} \sum_{p=1}^{\infty} n k_{j z}^{h} B_{n}\left(k_{\rho} \rho\right) e^{i n \phi}\left[e^{ \pm i k_{j z}^{h} z}-\tilde{R}_{j, j \mp 1}^{T E^{(z)}} e^{\mp i k_{j z}^{h}\left(z+2 z^{ \pm}\right)}\right]
\end{aligned}
$$

and we have for the TM mode

$$
\begin{aligned}
& E_{j \rho}=\frac{i k_{\rho} A_{j}^{ \pm e}}{k_{\rho_{T}}^{2}} \sum_{n=-\infty}^{\infty} \sum_{p=1}^{\infty} k_{j z}^{e} B_{n}^{\prime}\left(k_{\rho} \rho\right) e^{i n \phi}\left[e^{ \pm i k_{j z}^{e} z}-\tilde{R}_{j, j \mp 1}^{T M^{(z)}} e^{\mp i k_{j z}^{e}\left(z+2 z^{ \pm}\right)}\right] \\
& E_{j \phi}=-\frac{A_{j}^{ \pm e}}{k_{\rho_{T}}^{2} \rho} \sum_{n=-\infty}^{\infty} \sum_{p=1}^{\infty} n k_{j z}^{e} B_{n}\left(k_{\rho} \rho\right) e^{i n \phi}\left[e^{ \pm i k_{j z}^{e} z}-\tilde{R}_{j, j \mp 1}^{T M^{(z)}} e^{\mp i k_{j z}^{e}\left(z+2 z^{ \pm}\right)}\right] \\
& H_{j \rho}=\frac{\omega \epsilon_{j s} A_{j}^{ \pm e}}{k_{\rho_{T}}^{2} \rho} \sum_{n=-\infty}^{\infty} \sum_{p=1}^{\infty} n B_{n}\left(k_{\rho} \rho\right) e^{i n \phi}\left[e^{ \pm i k_{j z}^{e} z}+\tilde{R}_{j, j \mp 1}^{T M^{(z)}} e^{\mp i k_{j z}^{e}\left(z+2 z^{ \pm}\right)}\right] \\
& H_{j \phi}=\frac{i \omega \epsilon_{j s} k_{\rho} A_{j}^{ \pm e}}{k_{\rho_{T}}^{2}} \sum_{n=-\infty}^{\infty} \sum_{p=1}^{\infty} B_{n}^{\prime}\left(k_{\rho} \rho\right) e^{i n \phi}\left[e^{ \pm i k_{j z}^{e} z}+\tilde{R}_{j, j \mp 1}^{T M^{(z)}} e^{\mp i k_{j z}^{e}\left(z+2 z^{ \pm}\right)}\right]
\end{aligned}
$$

where $z^{ \pm}$is given by the equation (3-42). Notice that, in order to simplify the notation, we have omitted the subscript $n p$ in the radial and modal propagation constants. 


\section{4 \\ Electromagnetic Fields in Horizontal Stratified Media}

\section{1 \\ Introduction}

Electromagnetic fields in explored media in oil well prospecting are approached in this chapter. Axially-stratified isotropic and anisotropic formations will be considered. The fields in the propagation direction of the axial domain will be represented from the final expressions obtained in the previous chapter for decoupled TE and TM modes. For computing the fields, it will be necessary to find from the boundary conditions of our problem, the local and generalized reflection and transmission coefficients, which allows us to incorporate multiple layers in the media and describe the fields. Then, the propagation constants that give us the solution to problem are calculated from our characteristic equation. Finally, the influence of conductivity in the formation is analyzed.

\section{2}

\section{Axial Discontinuities}

This section is dedicated to finding the Fresnel or local and generalized reflection and transmission coefficients. A axially-stratified waveguide shown in Fig. 4.1 is composed by $N+1$ layers and it is used as base geometry to deduce the equations in our boundary problem. Each layer is formed by a uniaxially anisotropy medium, characterized by $\overline{\bar{\epsilon}}_{j}$ and $\overline{\bar{\mu}}_{j}$ where $j=2,3, \ldots, N+1$ in the region of $0 \leq \phi \leq 2 \pi$ and $\rho$ constant.

\subsection{1}

\section{Local Reflection and Transmission Coefficients}

We have a propagation of waves in a planar border, in which we will derive the local reflection and transmission coefficients. From the boundary 


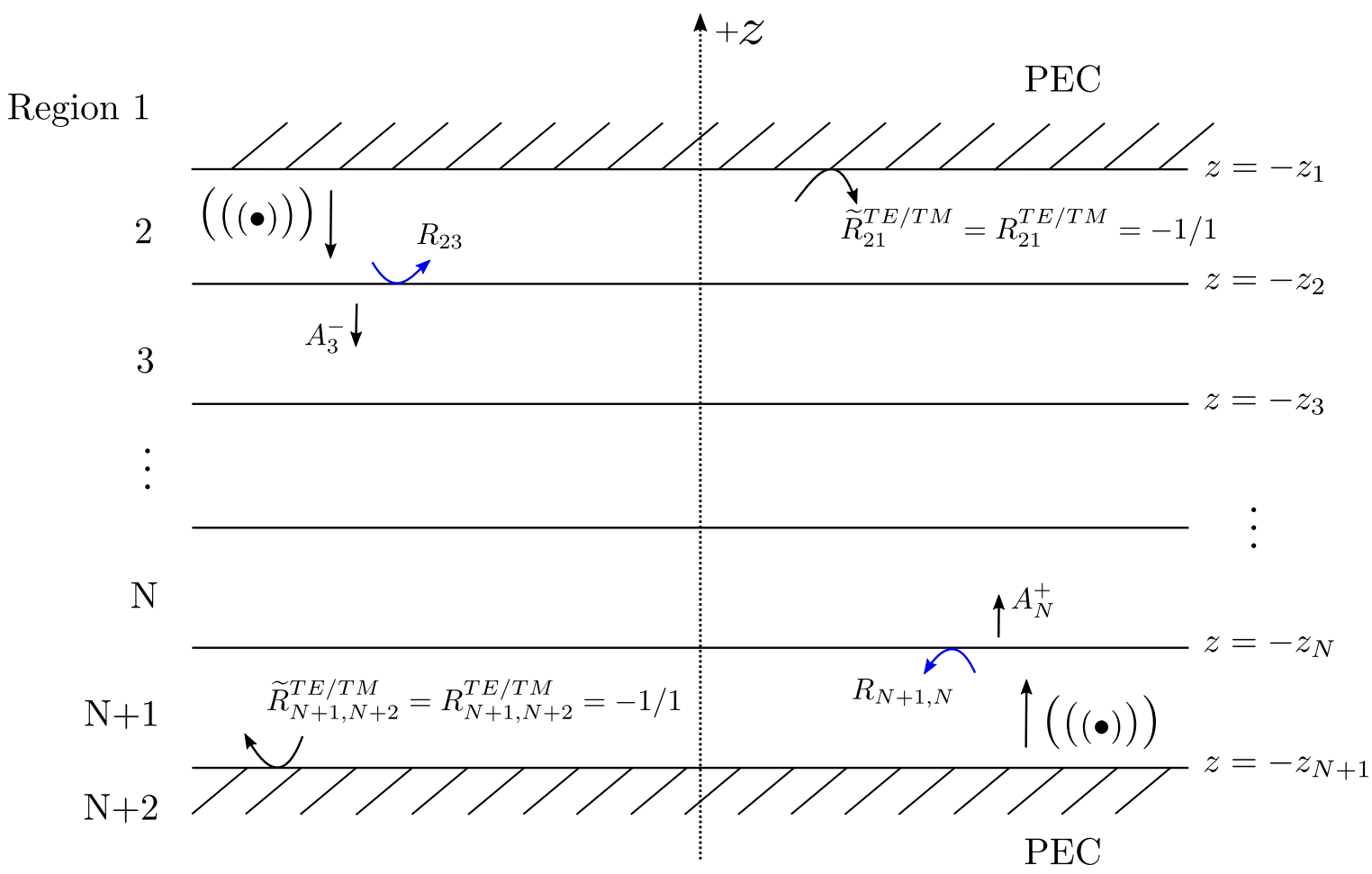

Figure 4.1: Front view of an axially-stratified $N+1$ - layers waveguide $(z$ in meters). The perfect electric conductor (PEC) is represented by the stripes.

conditions for a source-free region given by [2],

$$
\begin{array}{r}
-\hat{n} \times\left(\mathbf{E}_{3}-\mathbf{E}_{2}\right)=\mathbf{0} \\
\hat{n} \times\left(\mathbf{H}_{3}-\mathbf{H}_{2}\right)=\mathbf{0} \\
\hat{n} \cdot\left(\mathbf{D}_{3}-\mathbf{D}_{2}\right)=0 \\
\hat{n} \cdot\left(\mathbf{B}_{3}-\mathbf{B}_{2}\right)=0,
\end{array}
$$

in that $\hat{n}=\hat{n}_{23}$, which can be simplified in the $z=0$ interface to,

$$
\begin{aligned}
& \left.E_{2 \rho}\right|_{z=0}=\left.E_{3 \rho}\right|_{z=0} \\
& \left.E_{2 \phi}\right|_{z=0}=\left.E_{3 \phi}\right|_{z=0} \\
& \left.H_{2 \rho}\right|_{z=0}=\left.H_{3 \rho}\right|_{z=0} \\
& \left.H_{2 \phi}\right|_{z=0}=\left.H_{3 \phi}\right|_{z=0} .
\end{aligned}
$$

For the $T E^{z}$ mode the fields with $\partial / \partial \phi \rightarrow 0$ are,

$$
\begin{array}{r}
E_{\phi}=\frac{1}{k_{\rho}^{2}}\left(-i \omega \mu_{s} \frac{\partial H_{z}}{\partial \rho}\right) \\
H_{\rho}=\frac{1}{k_{\rho}^{2}}\left(i k_{z} \frac{\partial H_{z}}{\partial \rho}\right)
\end{array}
$$


with

$$
k_{z}= \pm \sqrt{k_{s}^{2}-k_{\rho}^{2}}
$$

where validating the conditions (4-6) and (4-7), we have the equations,

$$
\begin{aligned}
\mu_{2 s}\left(1+R_{23}^{T E(z)}\right) & =\mu_{3 s} T_{23}^{T E(z)} \\
k_{2 z}\left(-1+R_{23}^{T E(z)}\right) & =-k_{3 z} T_{23}^{T E(z)} .
\end{aligned}
$$

In general for a source radiating for $z \lessgtr 0$, from the above equations we can find the local reflection and transmission coefficients para the TE mode as,

$$
\begin{aligned}
R_{j, j \mp 1}^{T E(z)} & =\frac{k_{j z} \mu_{j \mp 1, s}-k_{j \mp 1, z} \mu_{j s}}{k_{j z} \mu_{j \mp 1, s}+k_{j \mp 1, z} \mu_{j s}} \\
T_{j, j \mp 1}^{T E E(z)} & =\frac{2 k_{j z} \mu_{j s}}{k_{j z} \mu_{j \mp 1, s}+k_{j \mp 1, z} \mu_{j s}} .
\end{aligned}
$$

The local reflection coefficient is the ratio of the amplitude of the reflected wave to the amplitude of the incident wave. The local transmission coefficient is the ratio of the amplitude of the transmitted wave to the amplitude of the incident wave [2].

For the $T M^{z}$ mode the fields with $\partial / \partial \phi=0$ are,

$$
\begin{array}{r}
E_{\rho}=\frac{1}{k_{\rho}^{2}}\left(i k_{z} \frac{\partial E_{z}}{\partial \rho}\right) \\
H_{\phi}=\frac{1}{k_{\rho}^{2}}\left(i \omega \epsilon_{s} \frac{\partial E_{z}}{\partial \rho}\right)
\end{array}
$$

with $k_{z}$ given by equation (4-11). Validating the conditions (4-5) and (4-8) or with a similar procedure to the previous one, we can find for a source radiating for $z \lessgtr 0$ in general, the local reflection and transmission coefficients for the TM mode as,

$$
\begin{aligned}
R_{j, j \mp 1}^{T M(z)} & =\frac{k_{j z} \epsilon_{j \mp 1, s}-k_{j \mp 1, z} \epsilon_{j s}}{k_{j z} \epsilon_{j \mp 1, s}+k_{j \mp 1, z} \epsilon_{j s}} \\
T_{j, j \mp 1}^{T M(z)} & =\frac{2 k_{j z} \epsilon_{j s}}{k_{j z} \epsilon_{j \mp 1, s}+k_{j \mp 1, z} \epsilon_{j s}} .
\end{aligned}
$$

Alternatively, the equations above can be derived from the duality principle with the equations (4-14) and (4-15), respectively.

We can verify that the local reflection and transmission coefficients for decoupled TE and TM modes represented by the equations (4-14), (4-15), (418) and (4-19) respectively, reduce to the ordinary Fresnel coefficients in [2] 
when the media becomes isotropic.

\subsection{2}

\section{Generalized Reflection Coefficients}

Assuming a source transmitting for $z<0$ as shown in Fig. 4.2, the downgoing wave in region 3 is a consequence of the transmission of the downgoing wave in region 2 plus a reflection of the upgoing wave in region 3 , this is,

$$
A_{3}^{-} e^{-i k_{3 z} z}=T_{23} A_{2}^{-} e^{-i k_{2 z} z}+R_{32} A_{3}^{-} R_{34} e^{i k_{3 z}\left(z+2 z_{3}\right)},
$$

where $R_{34}$ is a reflection coefficient that is the ratio of the upgoing wave amplitude and the downgoing wave amplitude at the interface $z=-z_{3}$. Note that the extra phase factor in the exponential that accompanies this coefficient ensures that this ratio is properly defined $[2,54]$.

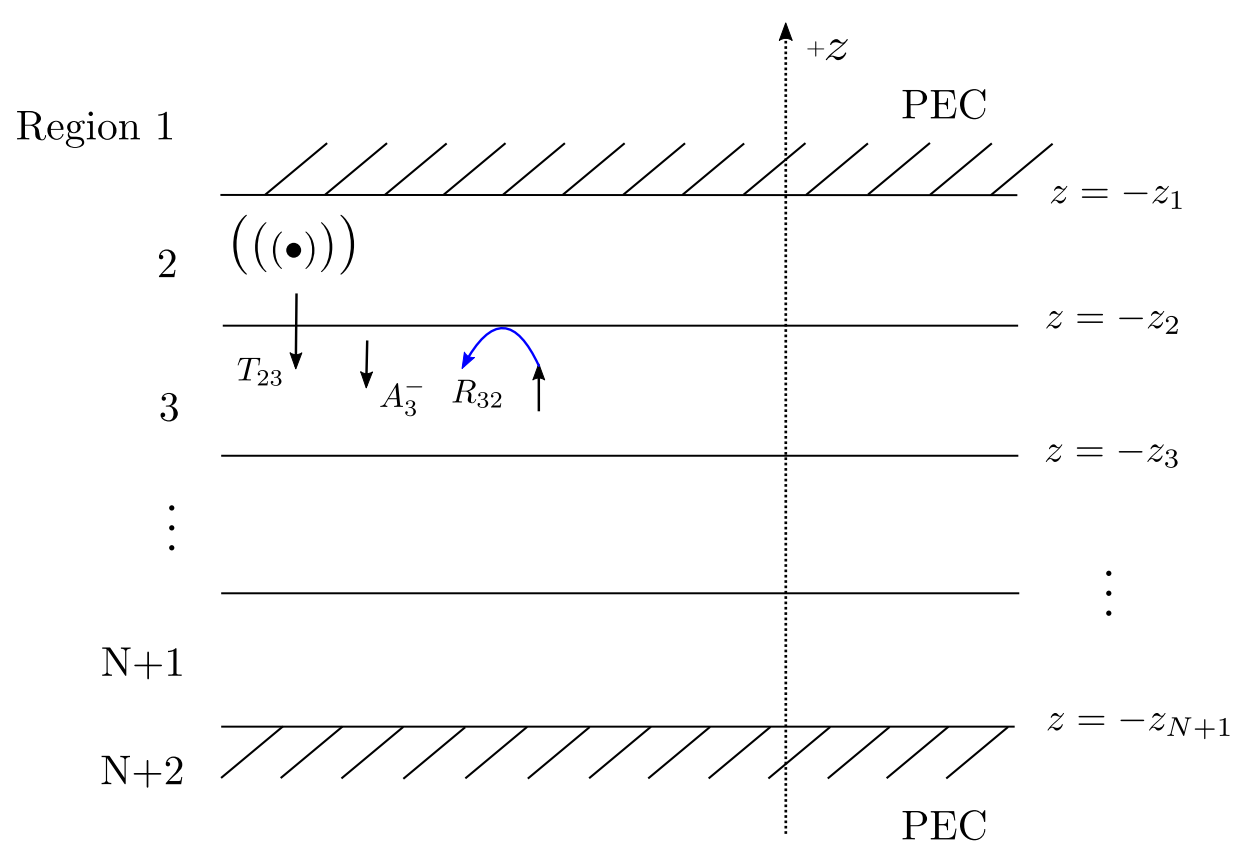

Figure 4.2: Front view of an axially-stratified $N+1$ - layers waveguide.

Evaluating the boundary condition at the $z=-z_{2}$ interface, from the equation (4-20), we get

$$
A_{3}^{-}=\frac{T_{23} A_{2}^{-} e^{i\left(k_{2 z}-k_{3 z}\right) z_{2}}}{1-R_{32} R_{34} e^{2 i k_{3 z}\left(z_{3}-z_{2}\right)}} .
$$

In the $z=-z_{2}$ interface we also have the upgoing wave in region 2 , which is caused by the reflection of the downgoing wave in region 2 plus a transmission of the upgoing wave in region 3 , this is,

$$
A_{2}^{-} \tilde{R}_{23} e^{i k_{2 z} z_{2}}=R_{23} A_{2}^{-} e^{i k_{2 z} z_{2}}+T_{32} A_{3}^{-} R_{34} e^{i k_{3 z}\left(-z_{2}+2 z_{3}\right)} .
$$

Substituting (4-21) into (4-22), we obtain 


$$
\tilde{R}_{23}=R_{23}+\frac{T_{23} R_{34} T_{32} e^{2 i k_{3 z}\left(z_{3}-z_{2}\right)}}{1-R_{32} R_{34} e^{2 i k_{3 z}\left(z_{3}-z_{2}\right)}} .
$$

After a some manipulations using the relationships $T_{i j}=1+R_{i j}$ and $R_{i j}=$ $-R_{j i}$ we have that the generalized reflection coefficient at the interface between region $j$ and region $j-1$ or $j+1$, can be written in general form as

$$
\tilde{R}_{j, j \mp 1}=\frac{R_{j, j \mp 1}+\tilde{R}_{j \mp 1, j \mp 2} e^{2 i k_{j \mp 1, z}\left(z_{j \mp 1}-\tilde{z}^{ \pm}\right)}}{1+R_{j, j \mp 1} \tilde{R}_{j \mp 1, j \mp 2} e^{2 i k_{j \mp 1, z}\left(z_{j \mp 1}-\tilde{z}^{ \pm}\right)}},
$$

where

$$
\tilde{z}^{ \pm}=\left\{\begin{array}{l}
\tilde{z}^{+}=z_{j-2} \\
\tilde{z}^{-}=z_{j}
\end{array}\right.
$$

This generalized reflection coefficient, $\tilde{R}_{j+1, j}$ for example, relates the amplitude of the downgoing wave to the amplitude of the upgoing wave in region $j+1$. It includes the effect of subsurface reflections as well as the reflection from the last interface [2].

\section{3}

\section{The Guidance Condition}

From the equation (3-41) we have that,

$$
\begin{aligned}
A_{j}^{-} e^{-i k_{j z} z} & =A_{j}^{+} \tilde{R}_{j, j-1} e^{-i k_{j z} z} e^{-2 i k_{j z} z_{j-1}} \quad \text { and } \\
A_{j}^{+} e^{i k_{j z} z} & =A_{j}^{-} \tilde{R}_{j, j+1} e^{i k_{j z} z} e^{2 i k_{j z} z_{j}} .
\end{aligned}
$$

Substituting (4-26) into (4-27) we can get our characteristic equation,

$$
f\left(k_{\rho}\right)=1-\tilde{R}_{j, j-1} \tilde{R}_{j, j+1} e^{2 i k_{j z}\left(z_{j}-z_{j-1}\right)}=0,
$$

similar to the one obtained in [1]. It allows us to find the eigenvalues $k_{\rho, n p}$ that satisfy the above equation and each one has a modal propagation constant associated $k_{z, n p}$.

For finding these eigenvalues, we modeled a simple case of a homogeneous medium into a parallel plate waveguide. In order to facilitate the validation to reader with the analytical case in Section 4.3.1, we assume a waveguide height of one meter, as shown the Fig. 4.3. We assume values of electrical permittivity and magnetic permeability equal to those of vacuum and a normalized source amplitude at $200 \mathrm{kHz}$ frequency. The Fig. 4.4 shows the resulting eigenvalues $k_{\rho, n p}$ after calculating the zeros of the equation (4-28). The Table 4.1 presents the results showed in Fig. 4.4. The eigenvalues presented in this Table shown the expected values proportional to $\pi$ and divided by the height of the layer, 


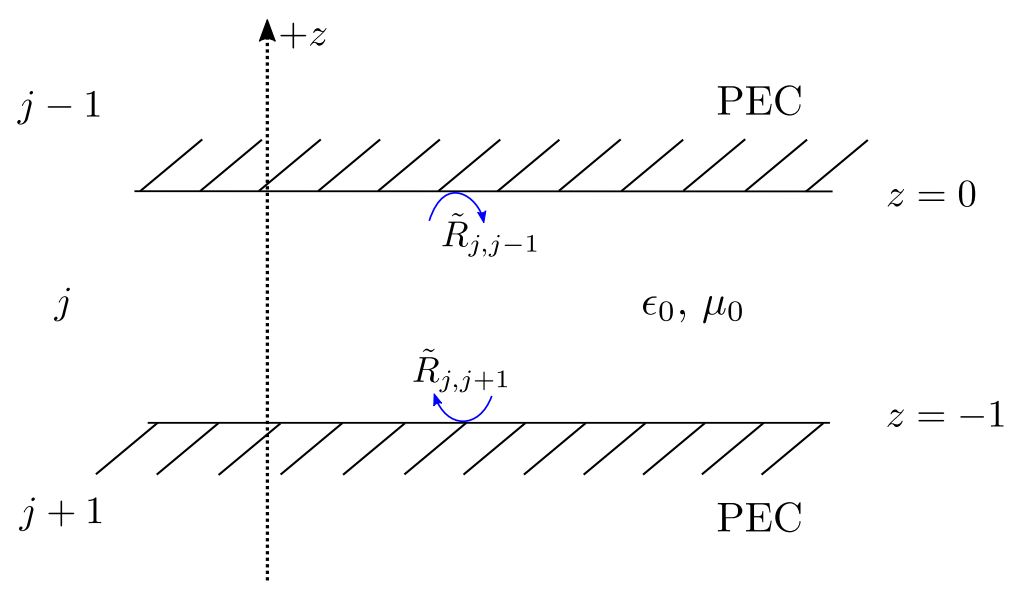

Figure 4.3: Front view of an parallel plate waveguide.

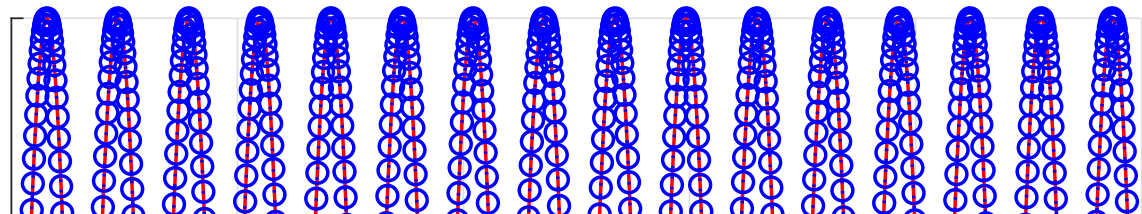

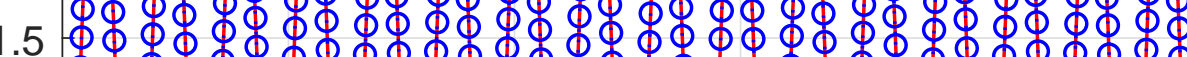

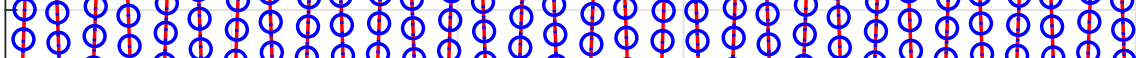

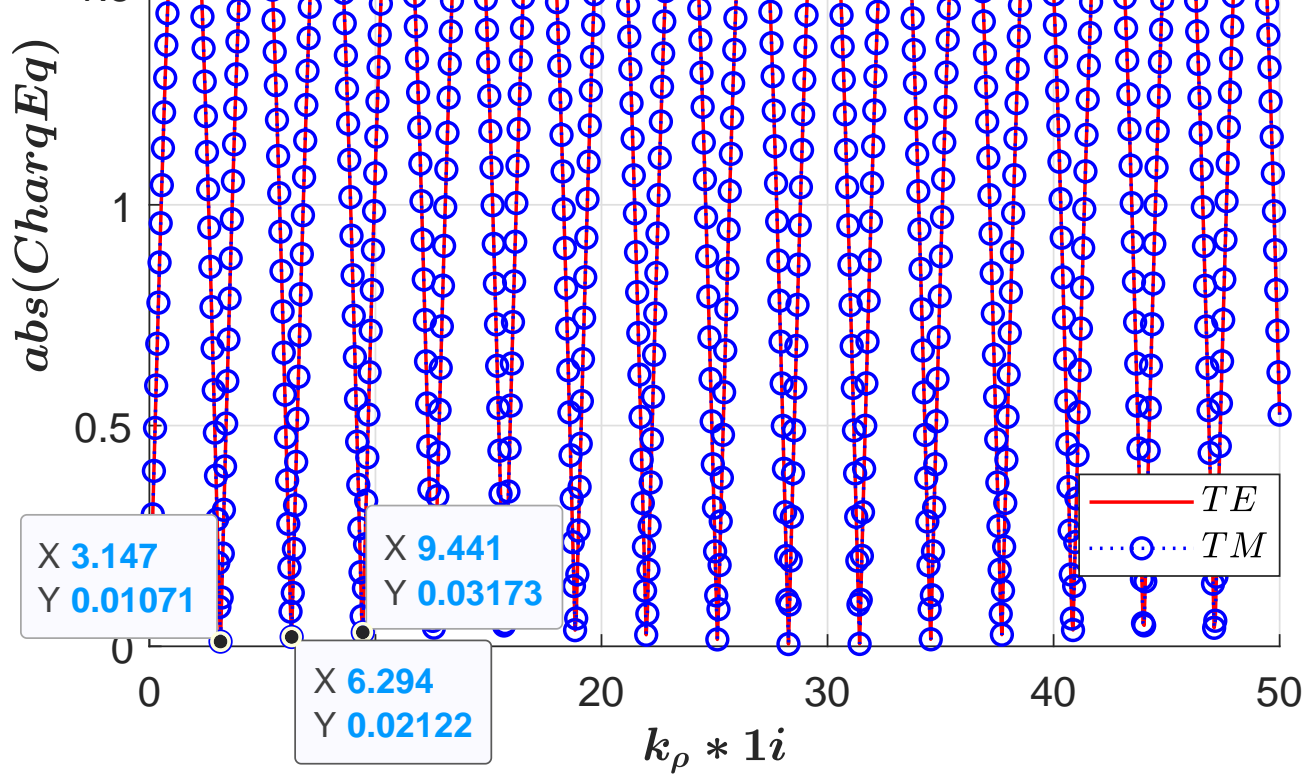

Figure 4.4: Characteristic equation evaluated along the imaginary $k_{\rho}$-axis.

Table 4.1: Eigenvalues for a parallel plate guide.

\begin{tabular}{c|c}
\hline \hline Mode Number & Eigenvalue $\boldsymbol{k}_{\boldsymbol{\rho}, \boldsymbol{n} \boldsymbol{p}}$ \\
\hline \hline 1st. & $3.141589856066267 \mathrm{i}$ \\
\hline 2nd. & $6.283183891768371 \mathrm{i}$ \\
\hline 3rd. & $9.424776972783139 \mathrm{i}$ \\
\hline
\end{tabular}


such as in the analytical solution for a parallel plate waveguide.

\subsection{1}

\section{Validation via Parallel Plate Waveguide}

In this section we will discuss the well-know analytical solution for a parallel plate waveguide. This result will be useful to validate the numerical solution obtained from the implemented algorithm.

In a parallel plate waveguide, we have a PEC interface at both the top and bottom interface. Due to this, when a wave propagates in this medium, the incident one on this kind of interface suffers a total internal reflection. In other words, all the energy of the incident wave is reflected. As a consequence, the magnitude of the reflection coefficients equals to unity at both interfaces. By substituting this value into (4-28) and making the height of the layer $D=z_{j}-z_{j-1}$, we obtain

$$
\begin{aligned}
1-e^{2 i k_{j z} D} & =0 \\
e^{i k_{j z} D}\left(e^{-i k_{j z} D}-e^{i k_{j z} D}\right) & =0 \\
2 i \sin \left(k_{j z} D\right) & =0 \\
k_{j z} & =\frac{n \pi}{D},
\end{aligned}
$$

where $n$ assumes positive and negative integer values.

We can appreciate the correspondence of the results obtained in Table 4.1 with the analytical solution in (4-29) to problem of a parallel plate waveguide.

\subsection{2}

\section{Pole-Free Characteristic Equation}

The generalized reflection coefficients of the characteristic equation (428) are in function of a local reflection coefficient, which in turn, contains a denominator equal to denominator of the local transmission coefficient. These denominators generate poles that must be removed from the characteristic equation, because they constitute a restriction to search for zeros. In other words, it is difficult to converge to a zero if it has poles in the vicinity. For example, if there is a pole $(\infty)$ very close to a zero, it can be that the zero is canceled or it is very difficult to get from one to another. Therefore, we have to remove the poles of the characteristic equation to stay only with the zeros of it, which are the ones that satisfy the contour problem.

Consequently, to locate all the poles for $N+1$ layers of our characteristic equation (4-28), we define for decoupled TE and TM modes the denominators 
of the characterized local reflection coefficients in Section 4.2.1 as

$$
\begin{aligned}
& D_{j a}^{T E(z)}=k_{j z} \mu_{j+1, s}+k_{j+1, z} \mu_{j, s}, \\
& D_{j b}^{T E(z)}=k_{j z} \mu_{j-1, s}+k_{j-1, z} \mu_{j, s}, \\
& D_{j a}^{T M(z)}=k_{j z} \epsilon_{j+1, s}+k_{j+1, z} \epsilon_{j, s}, \\
& D_{j b}^{T M(z)}=k_{j z} \epsilon_{j-1, s}+k_{j-1, z} \epsilon_{j, s} .
\end{aligned}
$$

Further of this denominators, as said before, the poles also come from the denominators of the generalized reflection coefficients of the equation (4-24). We define the following denominators for the generalized reflection coefficients $\tilde{R}_{j, j+1}$ and $\tilde{R}_{j, j-1}$ respectively,

$$
\begin{aligned}
D_{\text {minus }} & =1+R_{j, j+1} \tilde{R}_{j+1, j+2} e^{2 i k_{j+1, z}\left(z_{j+1}-z_{j}\right)} \\
D_{\text {plus }} & =1+R_{j, j-1} \tilde{R}_{j-1, j-2} e^{2 i k_{j-1, z}\left(z_{j-1}-z_{j-2}\right)} .
\end{aligned}
$$

The poles of the equation (4-28) can be removed using a deflationary process in which a new characteristic equation is defined as,

$$
f_{p f}\left(k_{\rho}\right)=f\left(k_{\rho}\right) f_{p}\left(k_{\rho}\right)
$$

The function $f_{p}\left(k_{\rho}\right)$ inserts zeros in the position of the poles of $f\left(k_{\rho}\right)$, in consequence, $f_{p f}\left(k_{\rho}\right)$ preserves the same zeros of $f\left(k_{\rho}\right)$, but is free of singularities [1].

Noting the denominators in (4-30) for the first and last layer, it is appropriate to define

$$
\begin{array}{ll}
\text { for } \quad R_{21}^{T E(z) / T M(z)}=-1 / 1 \quad \Rightarrow \quad D_{2 b}^{T E(z) / T M(z)}=1, \\
\text { for } \quad R_{N+1, N+2}^{T E(z) / T M(z)}=-1 / 1 \quad \Rightarrow \quad D_{N+1, a}^{T E(z) / T M(z)}=1 .
\end{array}
$$

Using the above definitions and after some manipulations, we can show that the zeros of

$$
f_{p}\left(k_{\rho}\right)=D_{2 b} D_{N+1, a} \prod_{j=2}^{N} D_{j a} D_{\text {minus }} \prod_{j=N+1}^{3} D_{j b} D_{\text {plus }}
$$

are the poles of $f\left(k_{\rho}\right)$. Substituting (4-28) and (4-35) in (4-32), we can write a pole free characteristic equation as 


$$
f_{p f}\left(k_{\rho}\right)=\prod_{j=2}^{N+1}\left[1-\tilde{R}_{j, j-1} \tilde{R}_{j, j+1} e^{2 i k_{j z}\left(z_{j}-z_{j-1}\right)}\right] \prod_{j=2}^{N} D_{j a} D_{\text {minus }} \prod_{j=N+1}^{3} D_{j b} D_{\text {plus }} .
$$

\section{4}

\section{Constant Amplitude $A_{j}^{ \pm}$}

\subsection{1}

\section{Source transmitting to positive $z$}

By considering a source transmitting for $z>0$ we have an upgoing wave in region $N$ in the $z=-z_{j}$ interface. This upgoing wave is caused by the reflection of the downgoing wave in region $N$ plus a transmission of the upgoing wave in region $N+1$, this is,

$$
A_{j}^{+} e^{-i k_{j z} z_{j}}=R_{j, j+1} A_{j}^{+} \tilde{R}_{j, j-1} e^{-i k_{j z}\left(-z_{j}+2 z_{j-1}\right)}+T_{j+1, j} A_{j+1}^{+} e^{-i k_{j+1, z} z_{j}}
$$

After a some manipulations in the above equation, we have that

$$
A_{j}^{+} e^{-i k_{j z} z_{j}}=A_{j+1}^{+} e^{-i k_{j+1, z} z_{j}} S_{j+1, j}
$$

where

$$
S_{j+1, j}=\frac{T_{j+1, j}}{1-R_{j, j+1} \tilde{R}_{j, j-1} e^{2 i k_{j z}\left(z_{j}-z_{j-1}\right)}} .
$$

From an iterative method of equation 4-38 and assuming $z_{N+1}=z_{N}$, we deduced that,

$$
A_{2}^{+} e^{-i k_{2 z} z_{2}}=A_{j+1}^{+} e^{-i k_{j+1, z} z_{j}} \prod_{j=N+1}^{3} e^{-i\left(z_{j-1}-z_{j}\right) k_{j z}} S_{j, j-1},
$$

where a generalized transmission coefficient can be defined by

$$
\tilde{T}_{N+1,2}=\prod_{j=N+1}^{3} e^{-i\left(z_{j-1}-z_{j}\right) k_{j z}} S_{j, j-1}
$$

in this way we find the amplitude $A_{j}^{+}$as

$$
A_{2}^{+} e^{-i k_{2 z} z_{2}}=\tilde{T}_{N+1,2} A_{N+1}^{+} e^{-i k_{N+1, z} z_{N}} .
$$

\subsection{2}

\section{Source transmitting to negative $z$}

In a similar procedure to the previous one but considering a source transmitting for $z<0$, we can get the amplitude $A_{j}^{-}$. From equation $4-21$ we obtain

$$
A_{j}^{-} e^{i k_{j z} z_{j-1}}=A_{j-1}^{-} e^{i k_{j-1, z} z_{j-1}} S_{j-1, j}
$$

where 


$$
S_{j-1, j}=\frac{T_{j-1, j}}{1-R_{j, j-1} \tilde{R}_{j, j+1} e^{2 i k_{j z}\left(z_{j}-z_{j-1}\right)}} .
$$

From an iterative method of equation $4-43$ and assuming $z_{1}=z_{2}$, we deduced that,

$$
A_{N+1}^{-} e^{i k_{N+1, z} z_{j}}=A_{2}^{-} e^{i k_{2 z} z_{2}} \prod_{j=2}^{N} e^{i\left(z_{j}-z_{j-1}\right) k_{j z}} S_{j, j+1},
$$

where a generalized transmission coefficient can be defined by

$$
\tilde{T}_{2, N+1}=\prod_{j=2}^{N} e^{i\left(z_{j}-z_{j-1}\right) k_{j z}} S_{j, j+1},
$$

in this way we find the amplitude $A_{j}^{-}$as

$$
A_{N+1}^{-} e^{i k_{N+1, z} z_{N}}=\tilde{T}_{2, N+1} A_{2}^{-} e^{i k_{2 z} z_{2}} .
$$

\section{5}

\section{Numerical Implementation and Analysis}

In order to implement the proposed method in this work, MATLAB numerical computing environment was used. For validating the initial part of algorithm, this section approaches several examples on lossless and lossy media. In both cases, some scenarios with both isotropic and isotropic layers are simulated. For all examples in this section was used a frequency of $200 \mathrm{kHz}$. We have considered an azimuthal index $n=1$ to excite all components of the electromagnetic fields, considering a coil source with fixed tilt angle of $45^{\circ}$, but with normalized amplitude. The contribution of the source will be analyzed in the next chapter. The fields will be observed throughout of the formations axial domain, which has a total length of 1 meter for validation purposes. Moreover, the fields will be represented for decoupled TE and TM modes due to truncation of our problem along of the axial domain.

\subsection{1}

\section{Lossless Media}

In this section non-conductive media are explored. For these media we consider isotropic and anisotropic formations. Furthermore, we validate the first three modes in each case, examining several examples with different amounts of layers to obtain a detailed validation of the implemented algorithm.

\subsubsection{1}

\section{Homogeneous Isotropic Formation}

In this first example was implemented the same problem of the Section 4.3 for a parallel plate waveguide as shown in Fig. 4.3. Therefore, the 
resulting eigenvalues are shown in Table 4.1. The propagation direction of the transmitted waves is towards positive $z$.

From Fig. 4.5 to Fig. 4.10, the first mode with $k_{\rho}=3.141589856066267 i$ was used for representing the electromagnetic fields. Fig. 4.5 shows the electric field for the radial component transversal to propagation direction. We can

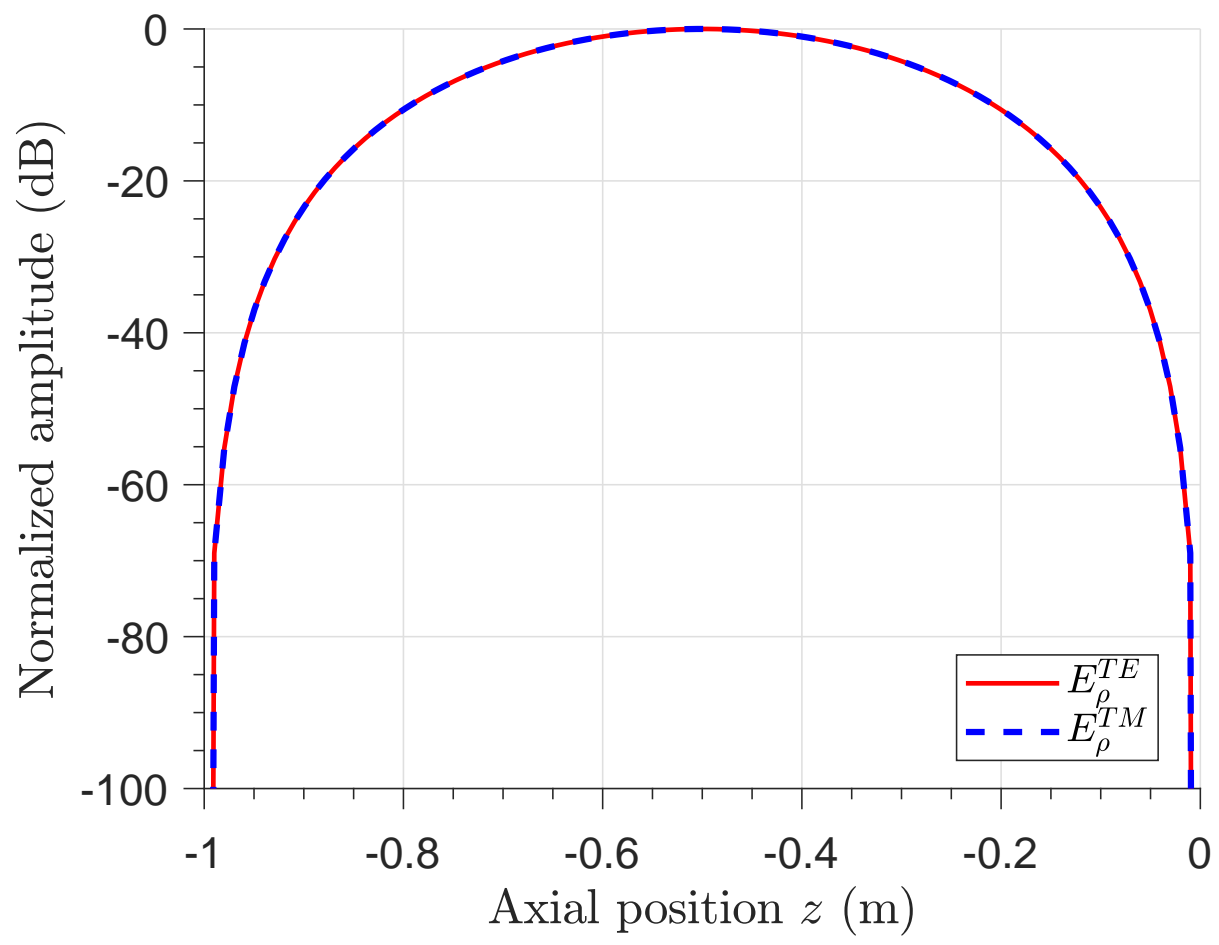

Figure 4.5: Normalized amplitude of the radial component of the electric field $\left(E_{\rho}\right)$ for decoupled TE and TM modes in a homogeneous and isotropic medium confined by a parallel plate waveguide (First Mode).

appreciate that both fields are maximum in center of waveguide and at the edges it goes to zero, which satisfies the boundary conditions of problem. In Fig. 4.6 is showed the magnetic field for radial component transversal to propagation direction. The electric and magnetic field for azimuthal component transversal to propagation direction are represented in Fig. 4.7 and 4.8, respectively. In Fig. 4.9 is showed the axial component of the electric and magnetic field in the propagation sense. Finally for the first mode, the electric and magnetic flux density onto $\hat{z}$ is presented in Fig. 4.10, which depend on electric and magnetic fields to $z$, respectively. We can notice how the boundary conditions of this problem are satisfied when at the edges of parallel plate guide the electric field to $\hat{z}$ is maximum and the magnetic field to $\hat{z}$ is minimum.

Similarly, all these fields are showed from Fig. 4.11 to Fig. 4.16. In this sense, they were simulated for the second eigenvalue $k_{\rho}=6.283183891768371 i$ from Table 4.1. 


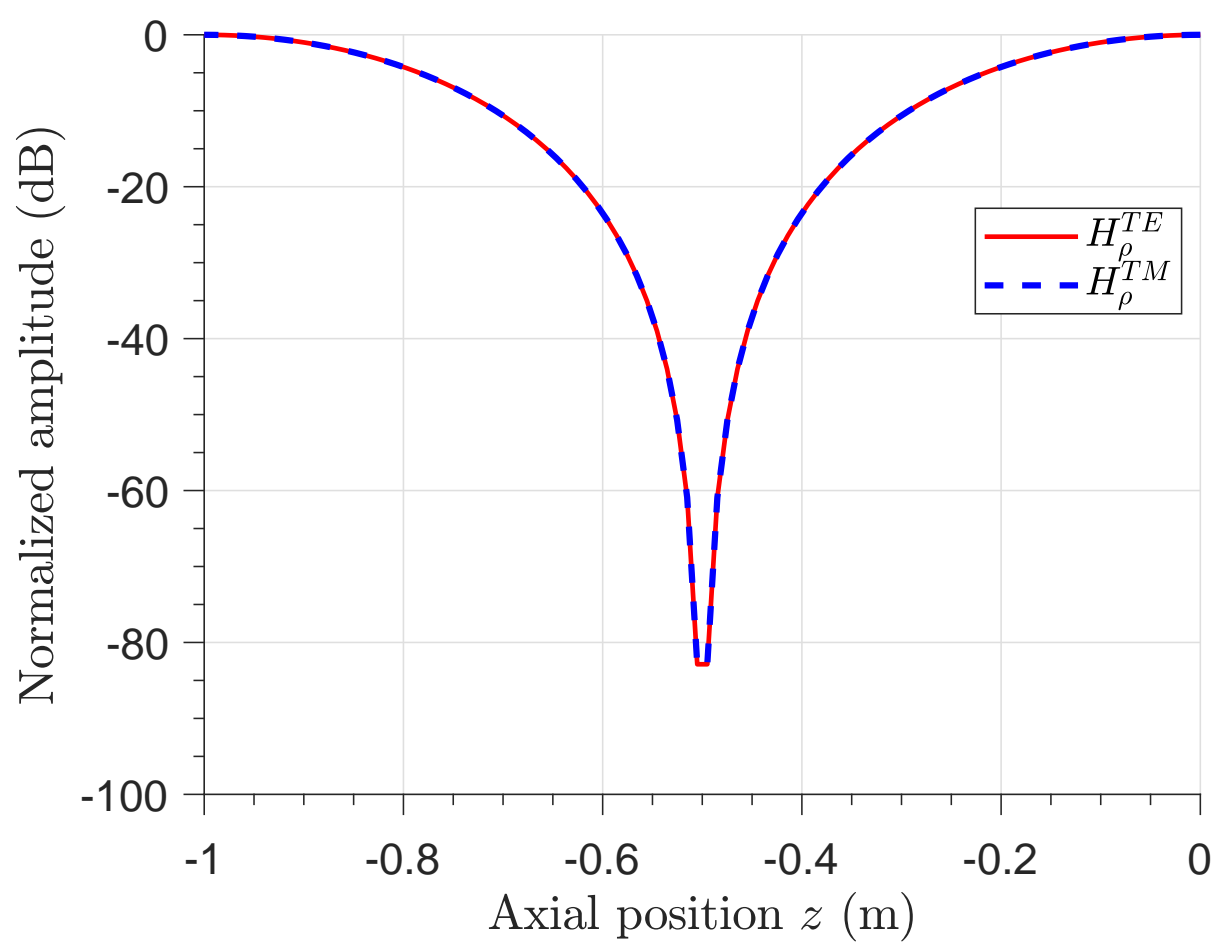

Figure 4.6: Normalized amplitude of the radial component of the magnetic field $\left(H_{\rho}\right)$ for decoupled TE and TM modes in a homogeneous and isotropic medium confined by a parallel plate waveguide (First Mode).

For the third mode with $k_{\rho}=9.424776972783139 i$, these six fields were also obtained, which are represented from Fig. 4.17 to Fig. 4.22.

\subsubsection{2}

\section{Half-Space Isotropic Formation}

In a second case of validation of algorithm, we implemented an isotropic Earth formation with two horizontal layers between the interfaces with $\hat{z}$ position $z=0, z=-0.5$ and $z=-1 \mathrm{~m}$, as shown in Fig. 4.23. The physical characteristics and eigenvalues of medium that provide the solution to our problem are shown in Table 4.2 and 4.3, respectively. The propagation

Table 4.2: Physical characteristics of an axially-stratified two-layers isotropic medium.

\begin{tabular}{c|c|c}
\hline \hline Layer & Relative Magnetic Permeability & Relative Electric Permittivity \\
\hline \hline 2 & $\mu_{r\{s, z\}}=1.15$ & $\epsilon_{r\{s, z\}}=1.8$ \\
\hline 3 & $\mu_{r\{s, z\}}=2.0$ & $\epsilon_{r\{s, z\}}=1.2$ \\
\hline \hline
\end{tabular}




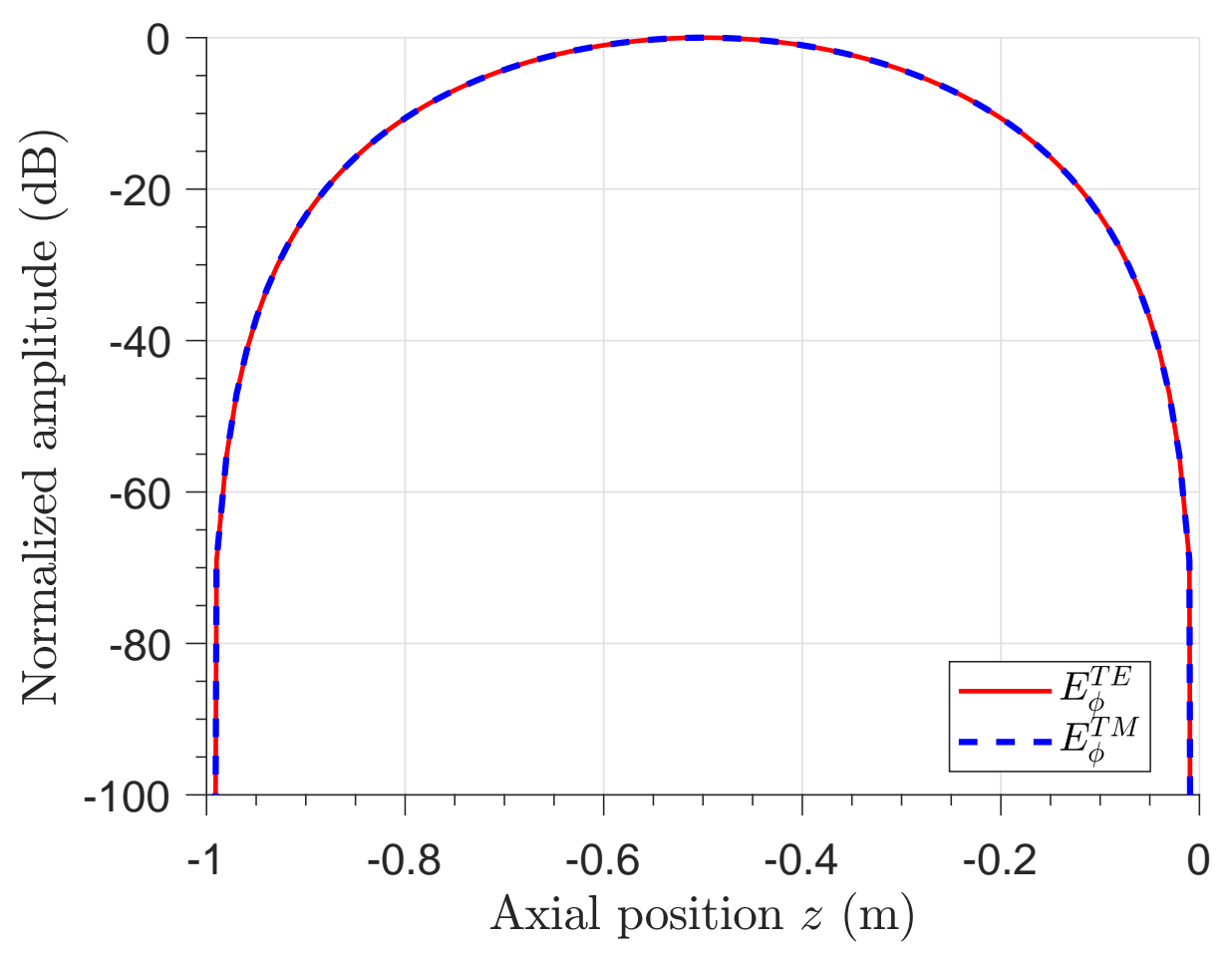

Figure 4.7: Normalized amplitude of the azimuthal component of the electric field $\left(E_{\phi}\right)$ for decoupled TE and TM modes in a homogeneous and isotropic medium confined by a parallel plate waveguide (First Mode).

Table 4.3: Eigenvalues for an axially-stratified two-layers isotropic medium.

\begin{tabular}{c|c|c}
\hline \hline \multirow{2}{*}{ Mode Number } & \multicolumn{2}{|c}{ Eigenvalue $\boldsymbol{k}_{\boldsymbol{\rho}, \boldsymbol{n} \boldsymbol{p}}$} \\
\cline { 2 - 3 } & TE & TM \\
\hline \hline 1st. & $3.141586520885489 \mathrm{i}$ & $3.141586492106696 \mathrm{i}$ \\
\hline 2nd. & $6.283182115262549 \mathrm{i}$ & $6.283182129630831 \mathrm{i}$ \\
\hline 3rd. & $9.424775688307591 \mathrm{i}$ & $9.424775755437505 \mathrm{i}$ \\
\hline \hline
\end{tabular}

direction of the transmitted waves was assumed towards the positive direction of the axial domain.

The first mode was used from Fig. 4.24 to Fig. 4.26 for representing the results of the electromagnetic fields for this scenario already described above. In the same way, the second mode was used from Fig. 4.27 to Fig. 4.31, and the third mode from Fig. 4.32 to Fig. 4.36. It is easily perceived that the boundary conditions are also satisfied at the medium edges as in the case of Section 4.5.1.1. An important point to discuss in this example is the notable difference in the $D_{z}$ amplitude to TM mode between the layer 2 and 3. This field concentration in layer 2 is due to a higher value of the relative electric permittivity in this layer with respect to the layer 3 , as is represented in Table 


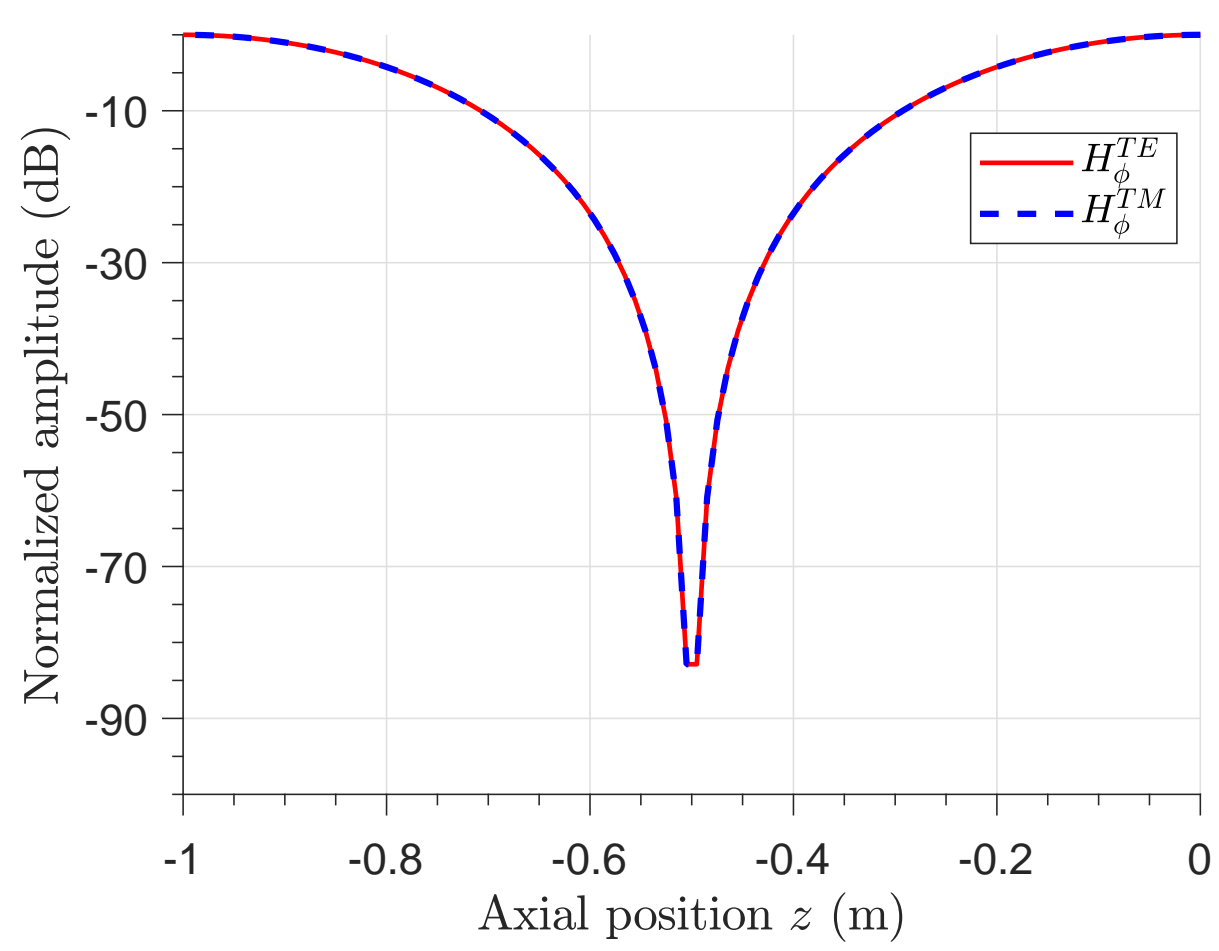

Figure 4.8: Normalized amplitude of the azimuthal component of the magnetic field $\left(H_{\phi}\right)$ for decoupled TE and TM modes in a homogeneous and isotropic medium confined by a parallel plate waveguide (First Mode).

4.2 .

\subsubsection{3}

\section{Multi-Layer Isotropic Formations}

\section{Three-Layer Isotropic Formation}

Another example for isotropic media is considered assuming three layers, which are found between the interfaces with $\hat{z}$-position $z=0, z=-0.3$, $z=-0.6$ and $z=-1 \mathrm{~m}$, as shown in Fig. 4.37. The physical characteristics of medium and some eigenvalues for this scenario are shown in Table 4.4 and Table 4.5, respectively. The propagation direction of the transmitted waves

Table 4.4: Physical characteristics of an axially-stratified three-layers isotropic medium.

\begin{tabular}{c|c|c}
\hline \hline Layer & Relative Magnetic Permeability & Relative Electric Permittivity \\
\hline \hline 2 & $\mu_{r\{s, z\}}=2.3$ & $\epsilon_{r\{s, z\}}=3.9$ \\
\hline 3 & $\mu_{r\{s, z\}}=1.2$ & $\epsilon_{r\{s, z\}}=1.4$ \\
\hline 4 & $\mu_{r\{s, z\}}=3.8$ & $\epsilon_{r\{s, z\}}=3.2$ \\
\hline \hline
\end{tabular}




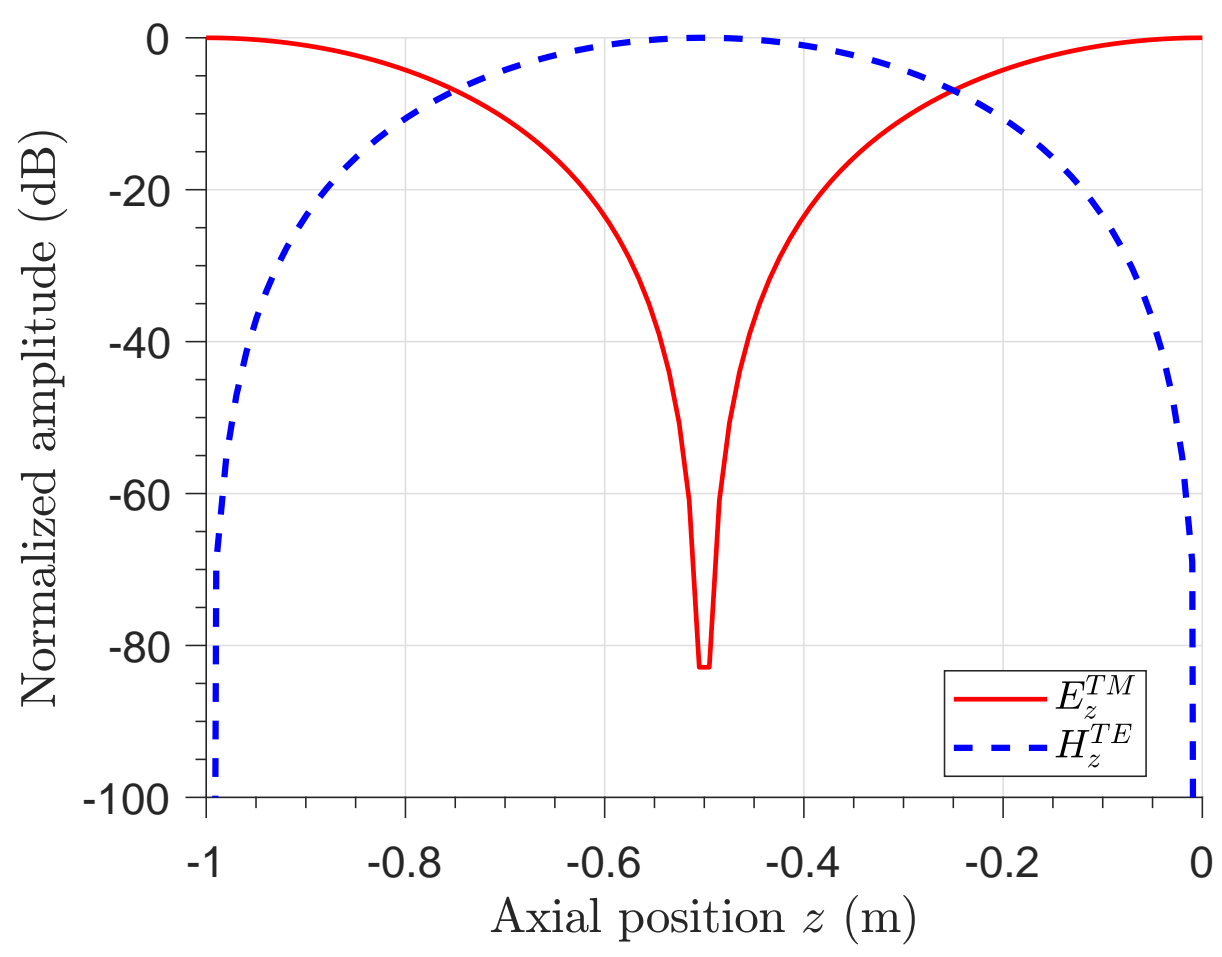

Figure 4.9: Normalized amplitude of the axial component of the electric and magnetic field $\left(E_{z}\right.$ and $\left.H_{z}\right)$ in a homogeneous and isotropic medium confined by a parallel plate waveguide (First Mode).

Table 4.5: Eigenvalues for an axially-stratified three-layers isotropic medium.

\begin{tabular}{c|c|c}
\hline \hline \multirow{2}{*}{ Mode Number } & \multicolumn{2}{|c}{ Eigenvalue $\boldsymbol{k}_{\boldsymbol{\rho}, \boldsymbol{n} \boldsymbol{p}}$} \\
\cline { 2 - 3 } & TE & TM \\
\hline \hline 1st. & $2.446160254330170 \mathrm{i}$ & $3.725765658926201 \mathrm{i}$ \\
\hline 2nd. & $7.016974738107960 \mathrm{i}$ & $5.670998168819509 \mathrm{i}$ \\
\hline 3rd. & $9.157010767173599 \mathrm{i}$ & $9.607950593823594 \mathrm{i}$ \\
\hline \hline
\end{tabular}

was assumed towards the negative sense of the axial domain.

In Fig. 4.38 and Fig. 4.39 we show the fields for the first mode, from Fig. 4.40 to Fig. 4.42 for the second mode, and from Fig. 4.43 to Fig. 4.45 for the third mode. 


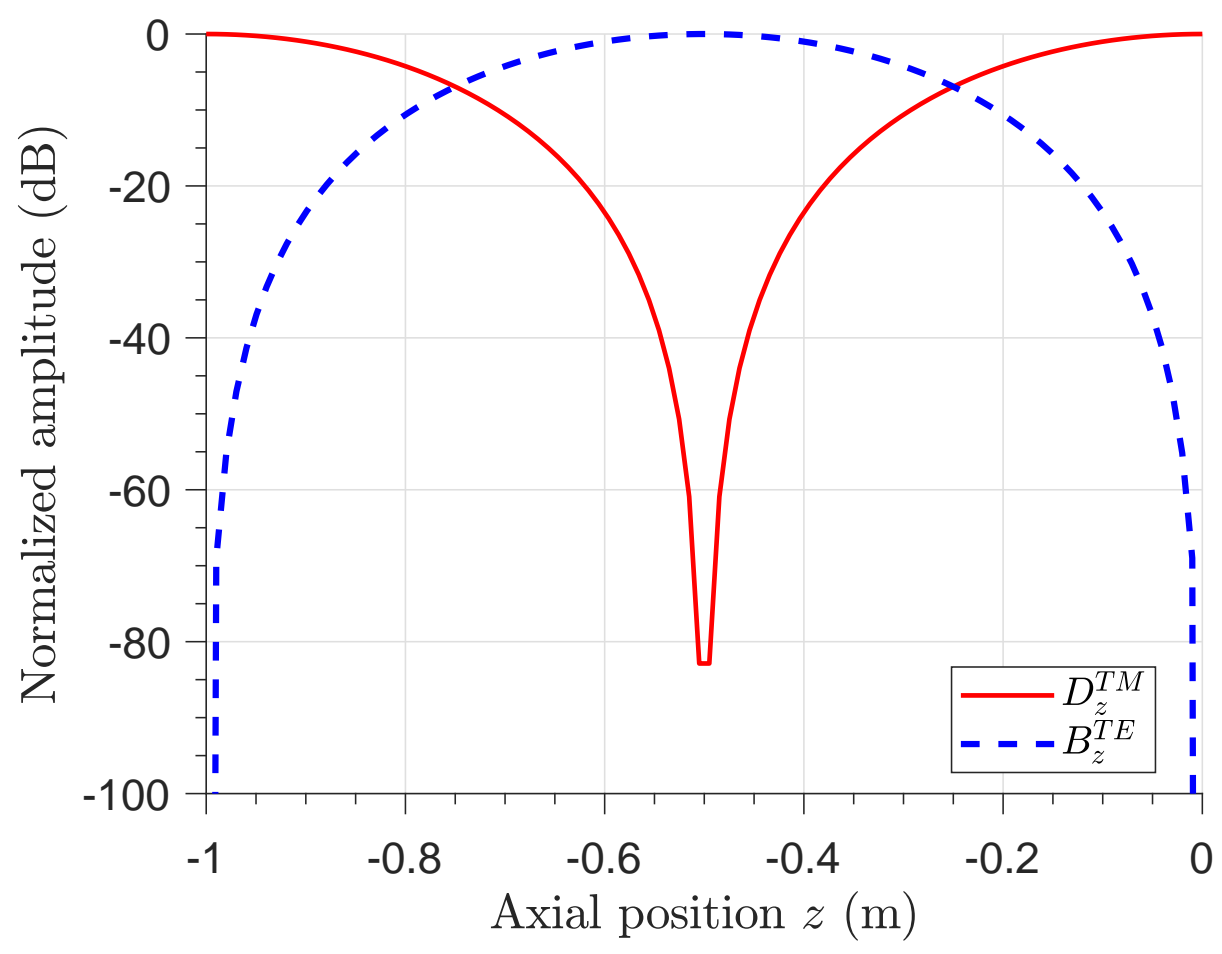

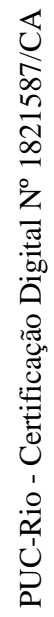

Figure 4.10: Normalized amplitude of the electric flux density and magnetic flux density $\left(D_{z}\right.$ and $\left.B_{z}\right)$ in a homogeneous and isotropic medium confined by a parallel plate waveguide (First Mode).

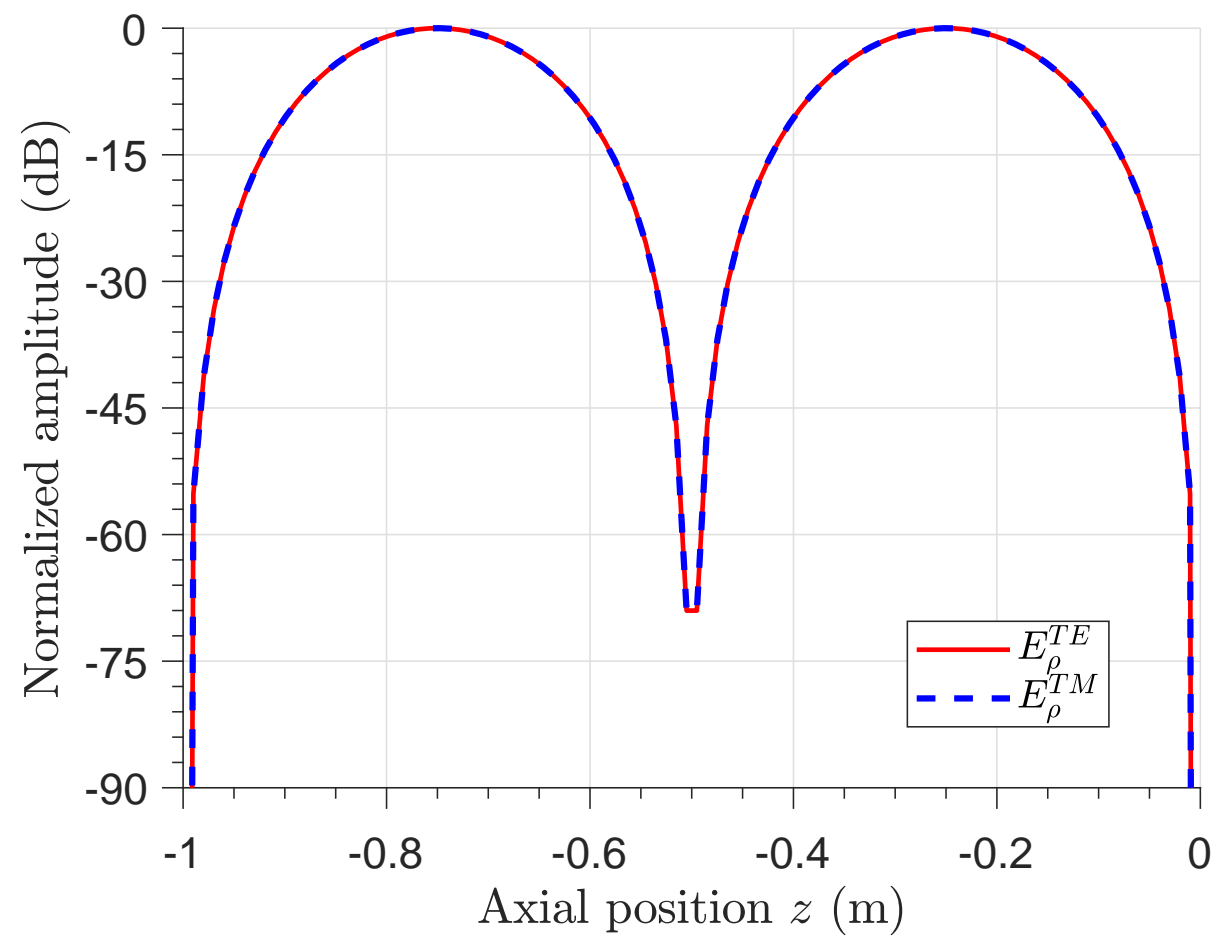

Figure 4.11: Normalized amplitude of the radial component of the electric field $\left(E_{\rho}\right)$ for decoupled TE and TM modes in a homogeneous and isotropic medium confined by a parallel plate waveguide (Second Mode). 


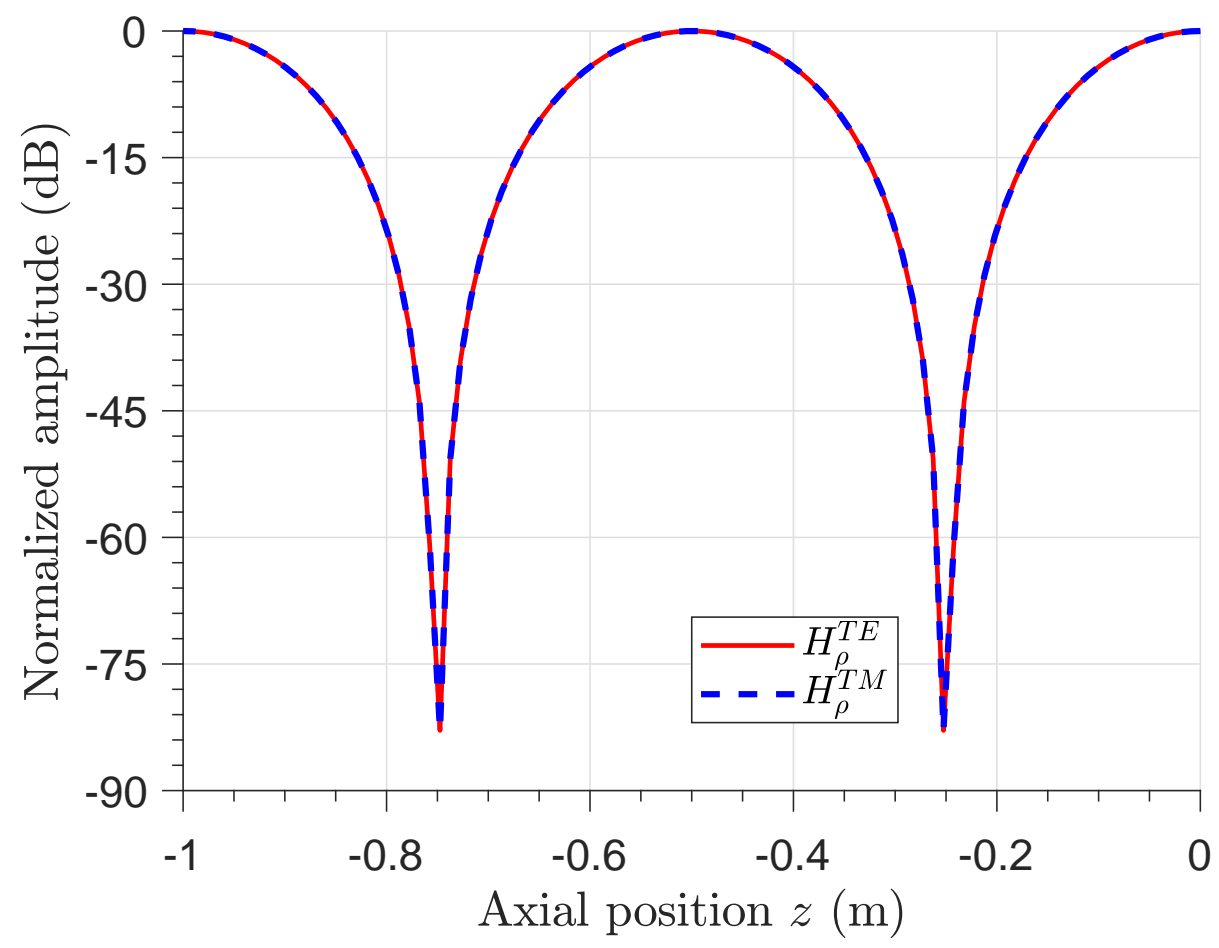

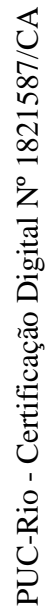

Figure 4.12: Normalized amplitude of the radial component of the magnetic field $\left(H_{\rho}\right)$ for decoupled TE and TM modes in a homogeneous and isotropic medium confined by a parallel plate waveguide (Second Mode).

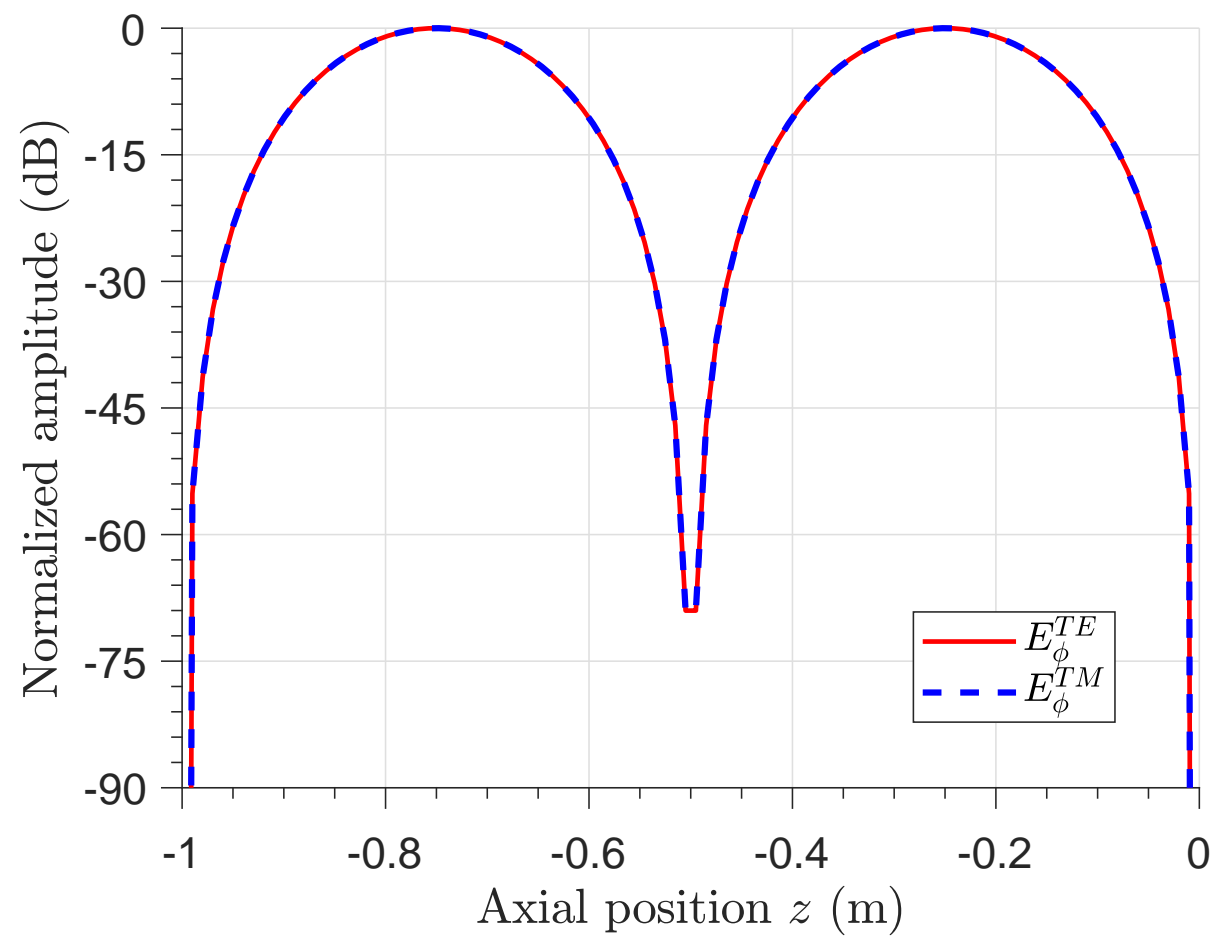

Figure 4.13: Normalized amplitude of the azimuthal component of the electric field $\left(E_{\phi}\right)$ for decoupled TE and TM modes in a homogeneous and isotropic medium confined by a parallel plate waveguide (Second Mode). 


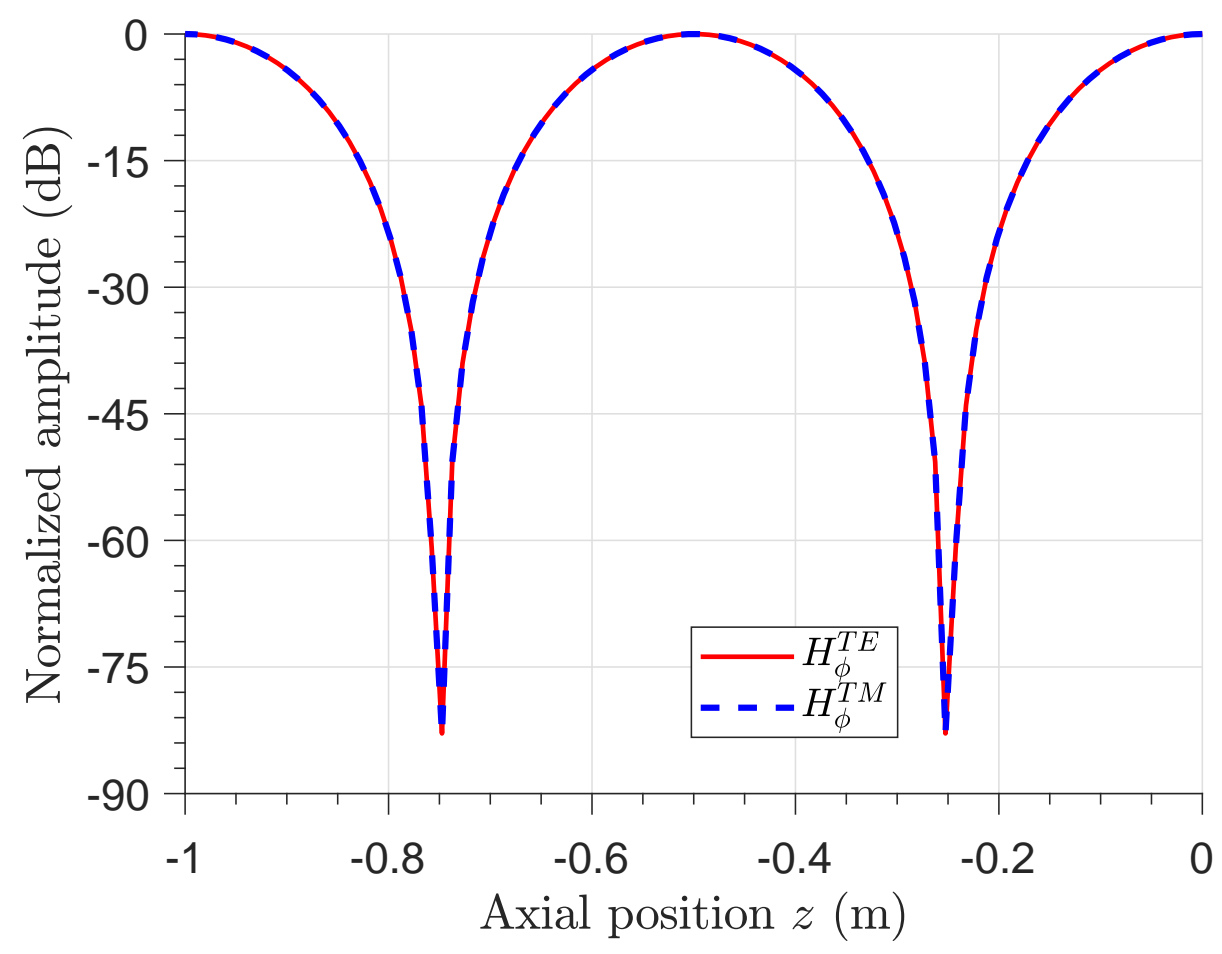

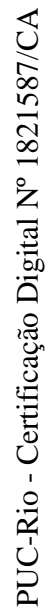

Figure 4.14: Normalized amplitude of the azimuthal component of the magnetic field $\left(H_{\phi}\right)$ for decoupled TE and TM modes in a homogeneous and isotropic medium confined by a parallel plate waveguide (Second Mode).

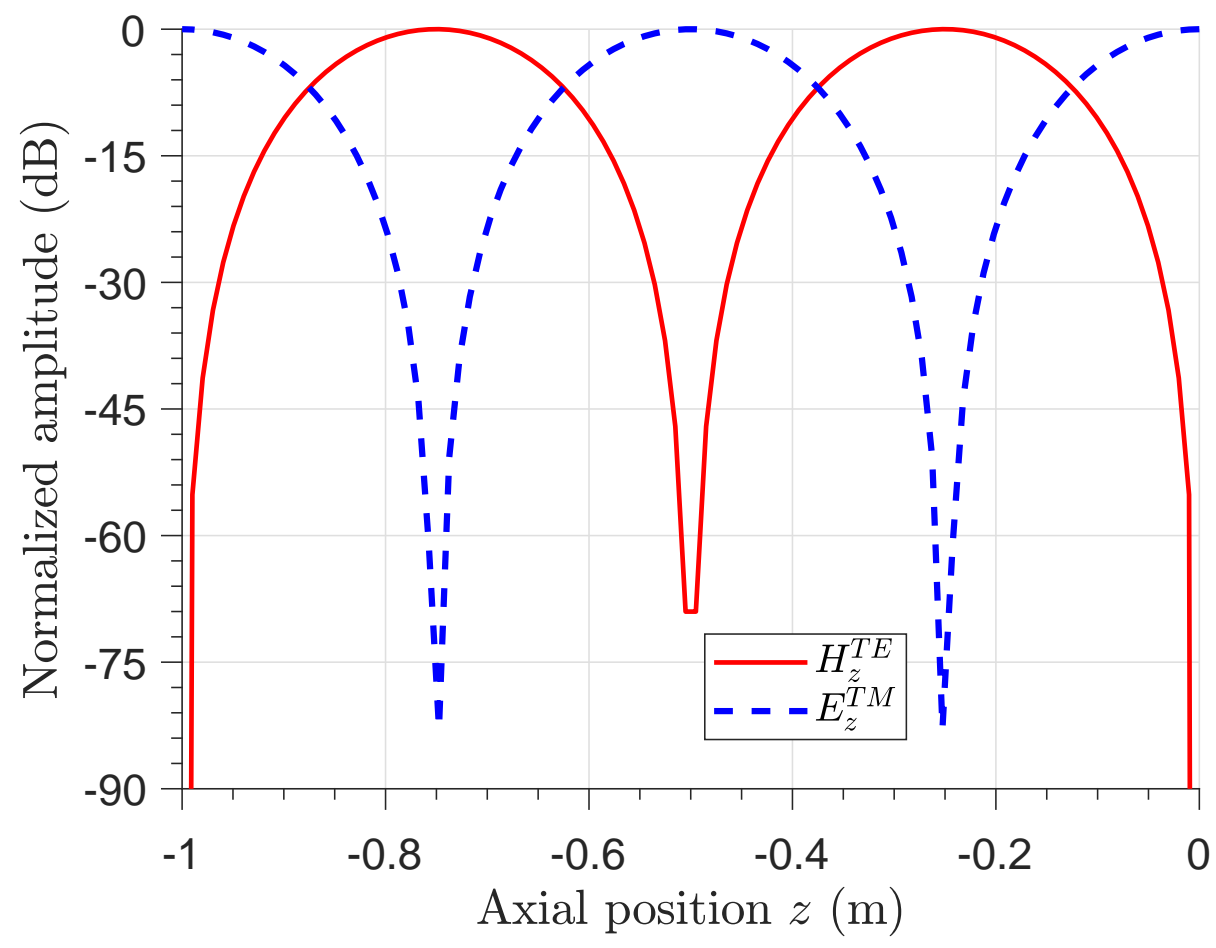

Figure 4.15: Normalized amplitude of the axial component of the electric and magnetic field $\left(E_{z}\right.$ and $\left.H_{z}\right)$ in a homogeneous and isotropic medium confined by a parallel plate waveguide (Second Mode). 


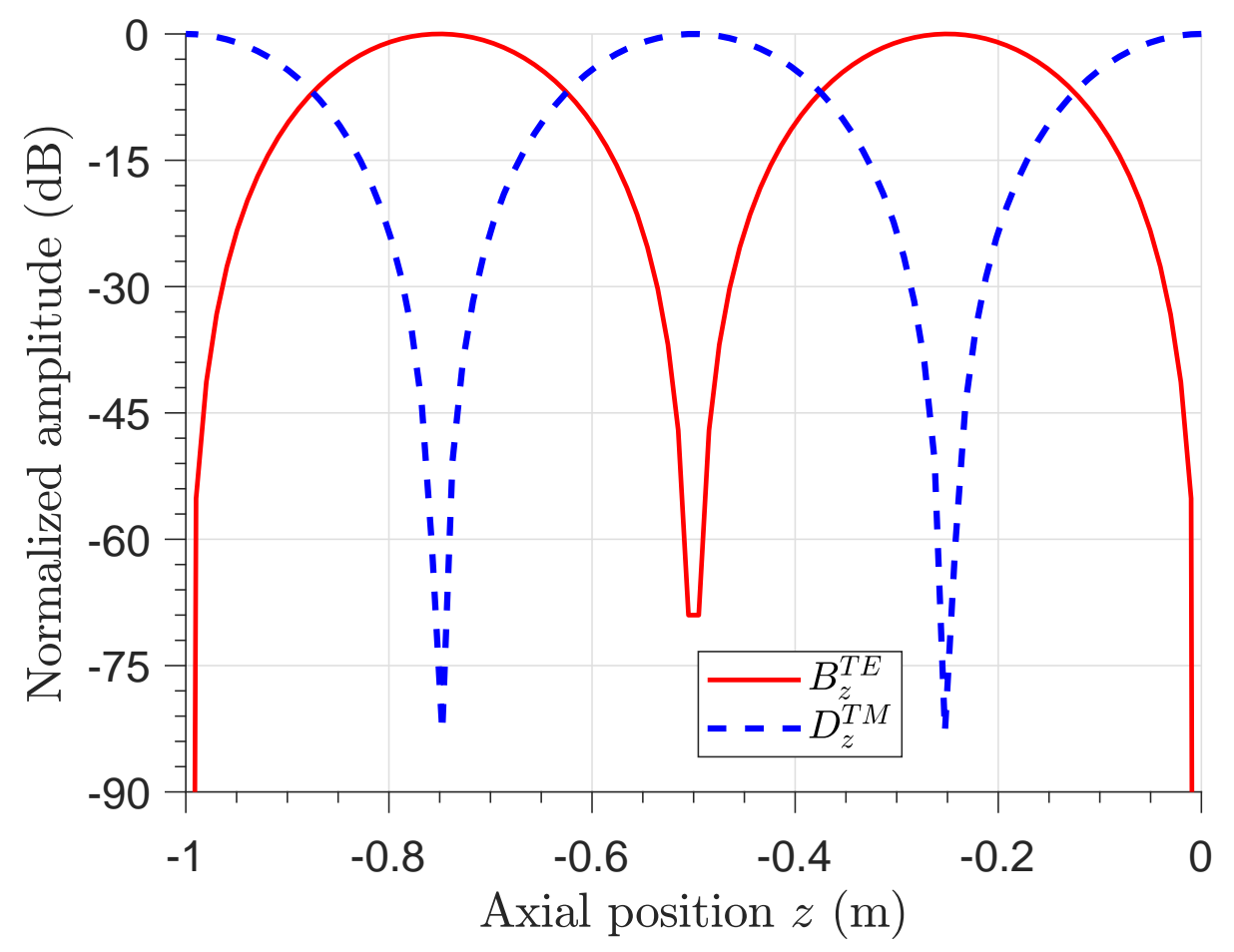

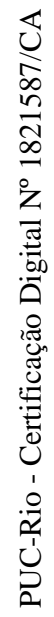

Figure 4.16: Normalized amplitude of the electric flux density and magnetic flux density $\left(D_{z}\right.$ and $\left.B_{z}\right)$ in a homogeneous and isotropic medium confined by a parallel plate waveguide (Second Mode).

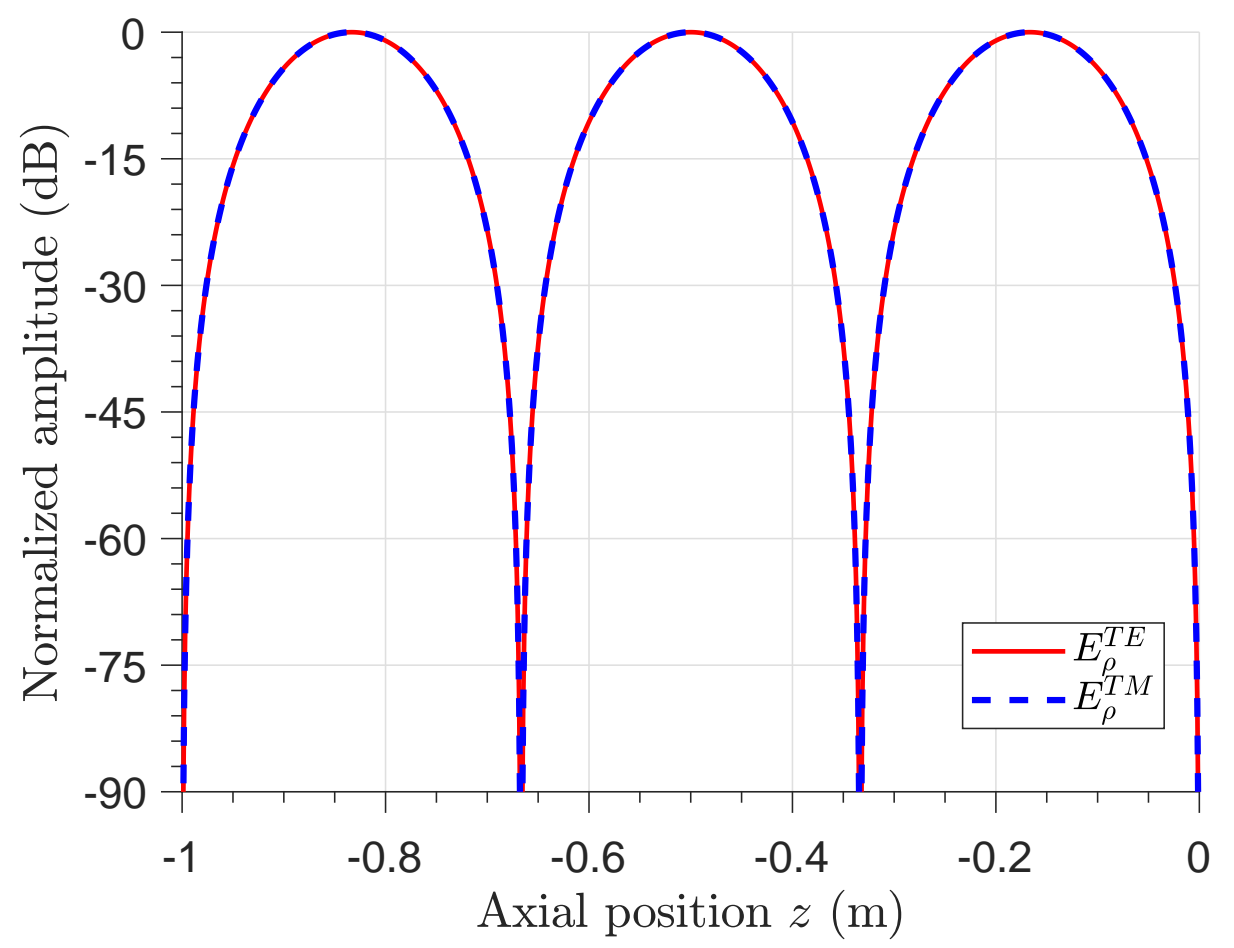

Figure 4.17: Normalized amplitude of the radial component of the electric field $\left(E_{\rho}\right)$ for decoupled TE and TM modes in a homogeneous and isotropic medium confined by a parallel plate waveguide (Third Mode). 


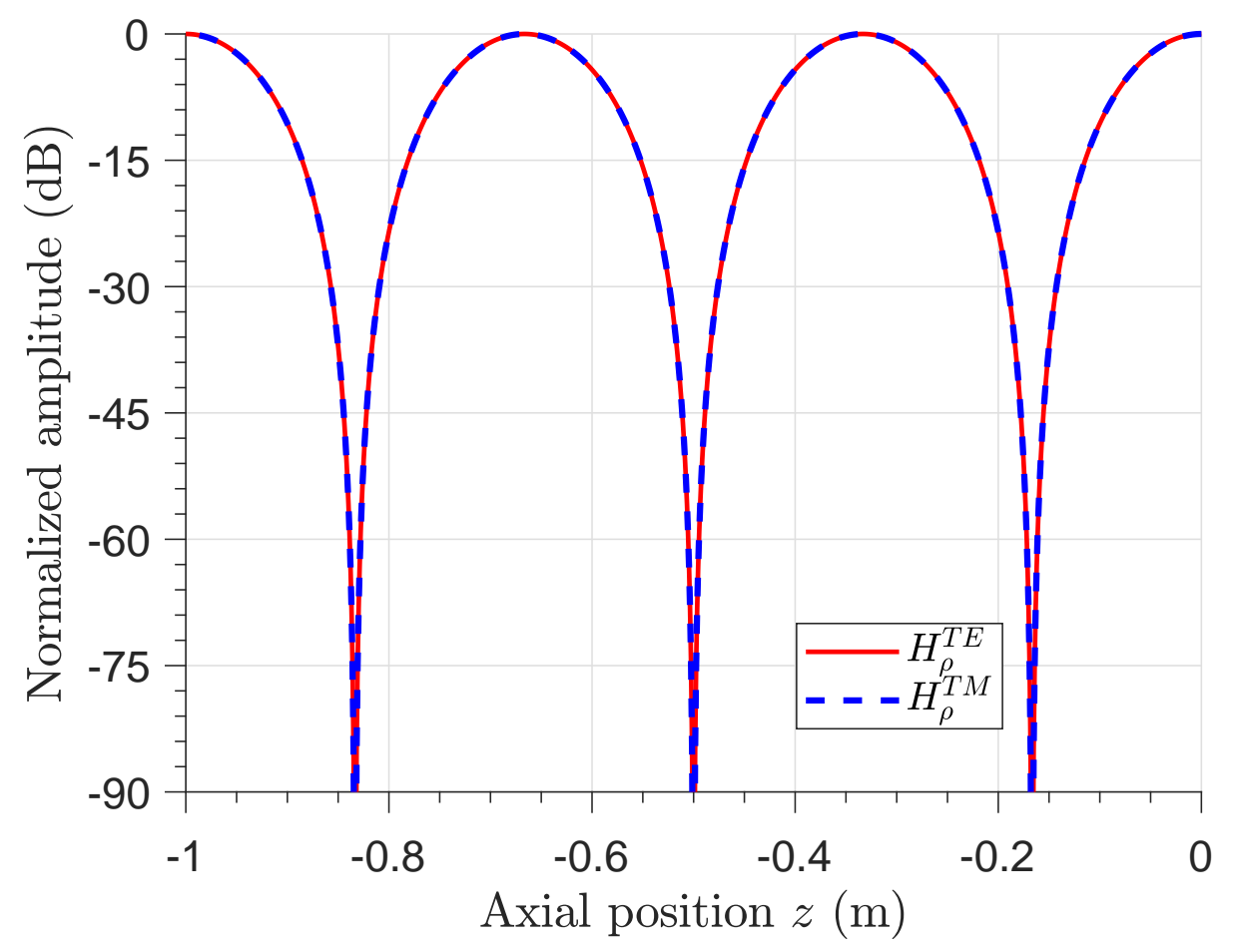

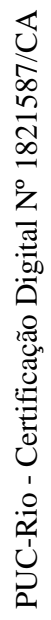

Figure 4.18: Normalized amplitude of the radial component of the magnetic field $\left(H_{\rho}\right)$ for decoupled TE and TM modes in a homogeneous and isotropic medium confined by a parallel plate waveguide (Third Mode).

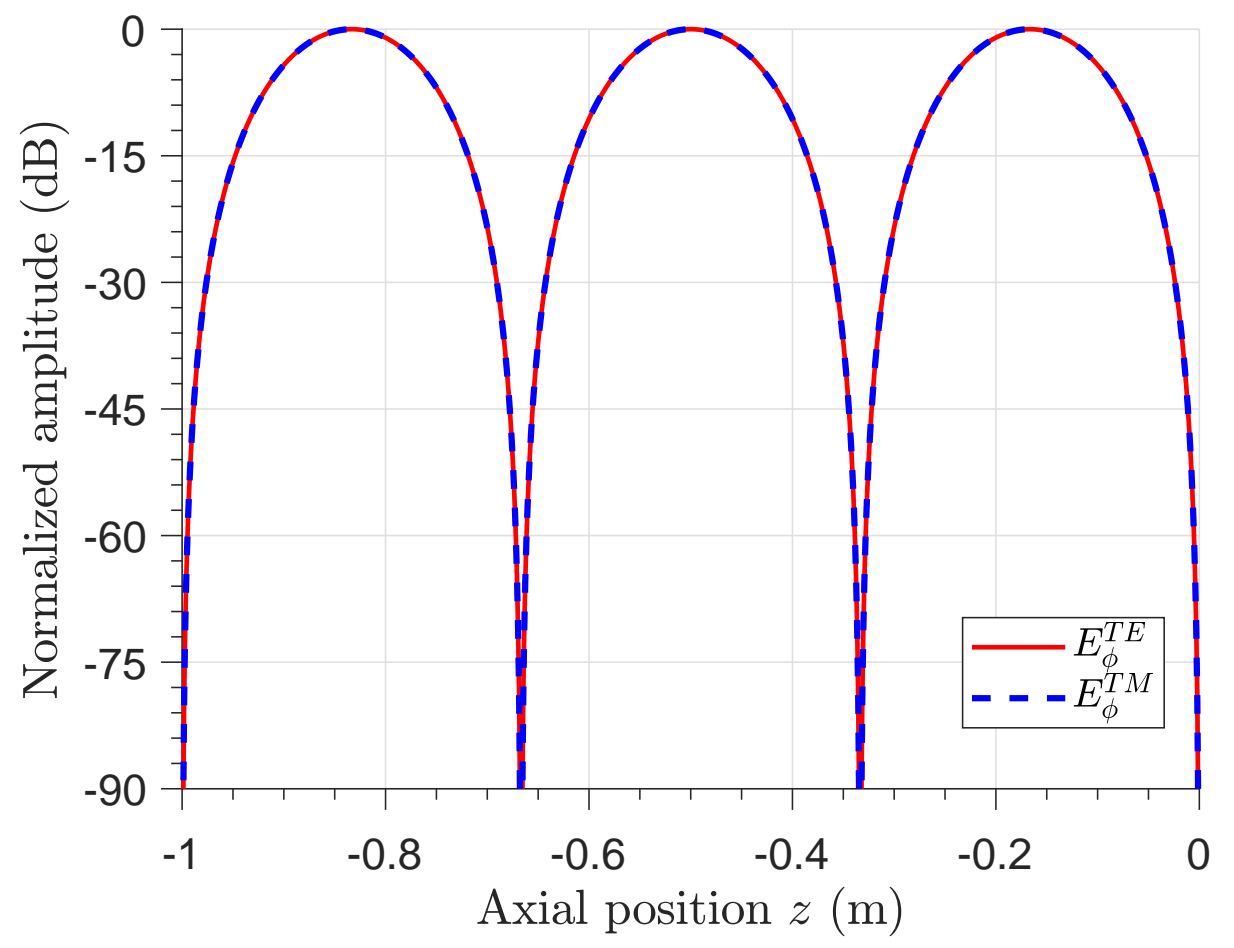

Figure 4.19: Normalized amplitude of the azimuthal component of the electric field $\left(E_{\phi}\right)$ for decoupled TE and TM modes in a homogeneous and isotropic medium confined by a parallel plate waveguide (Third Mode). 


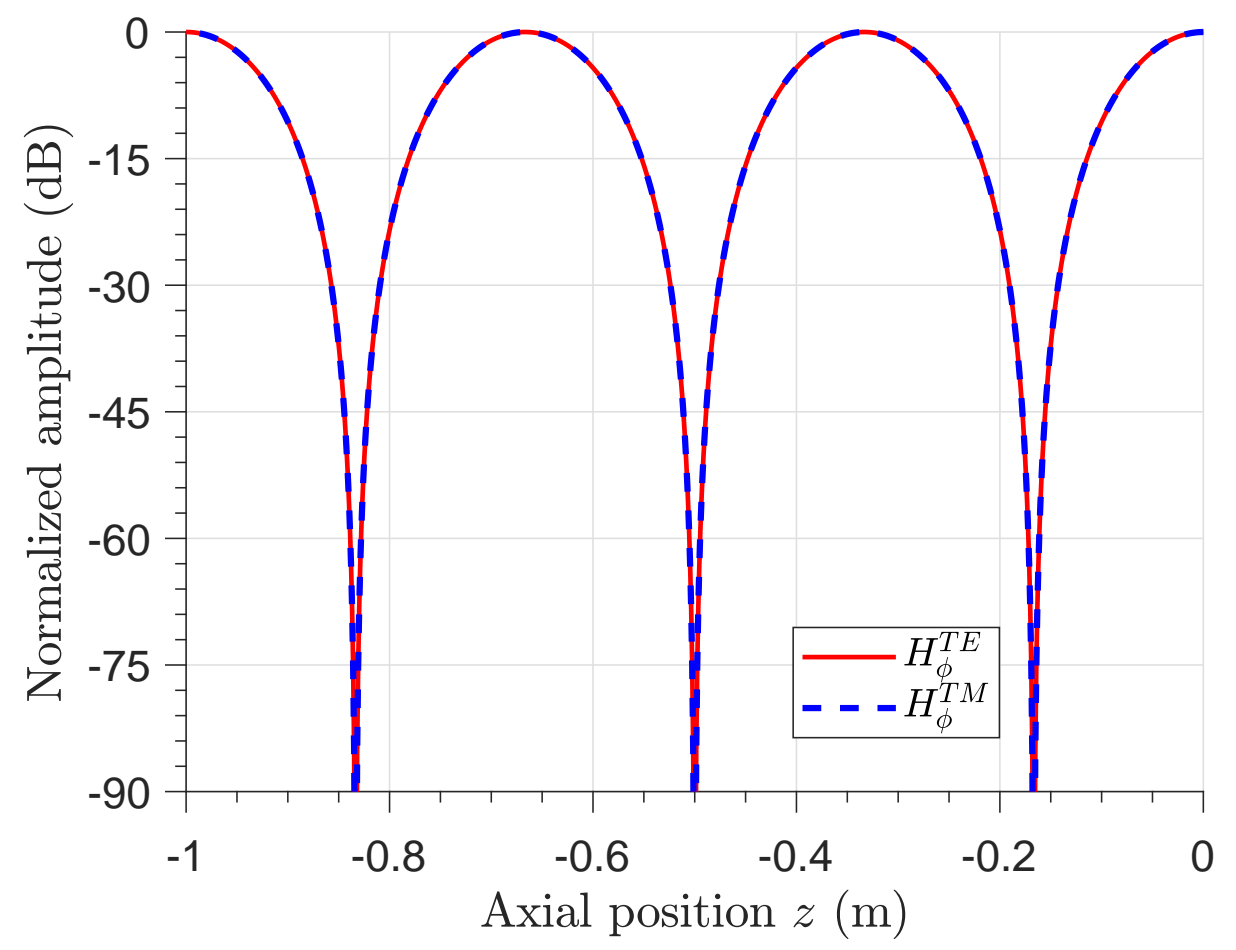

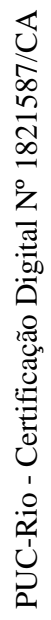

Figure 4.20: Normalized amplitude of the azimuthal component of the magnetic field $\left(H_{\phi}\right)$ for decoupled TE and TM modes in a homogeneous and isotropic medium confined by a parallel plate waveguide (Third Mode).

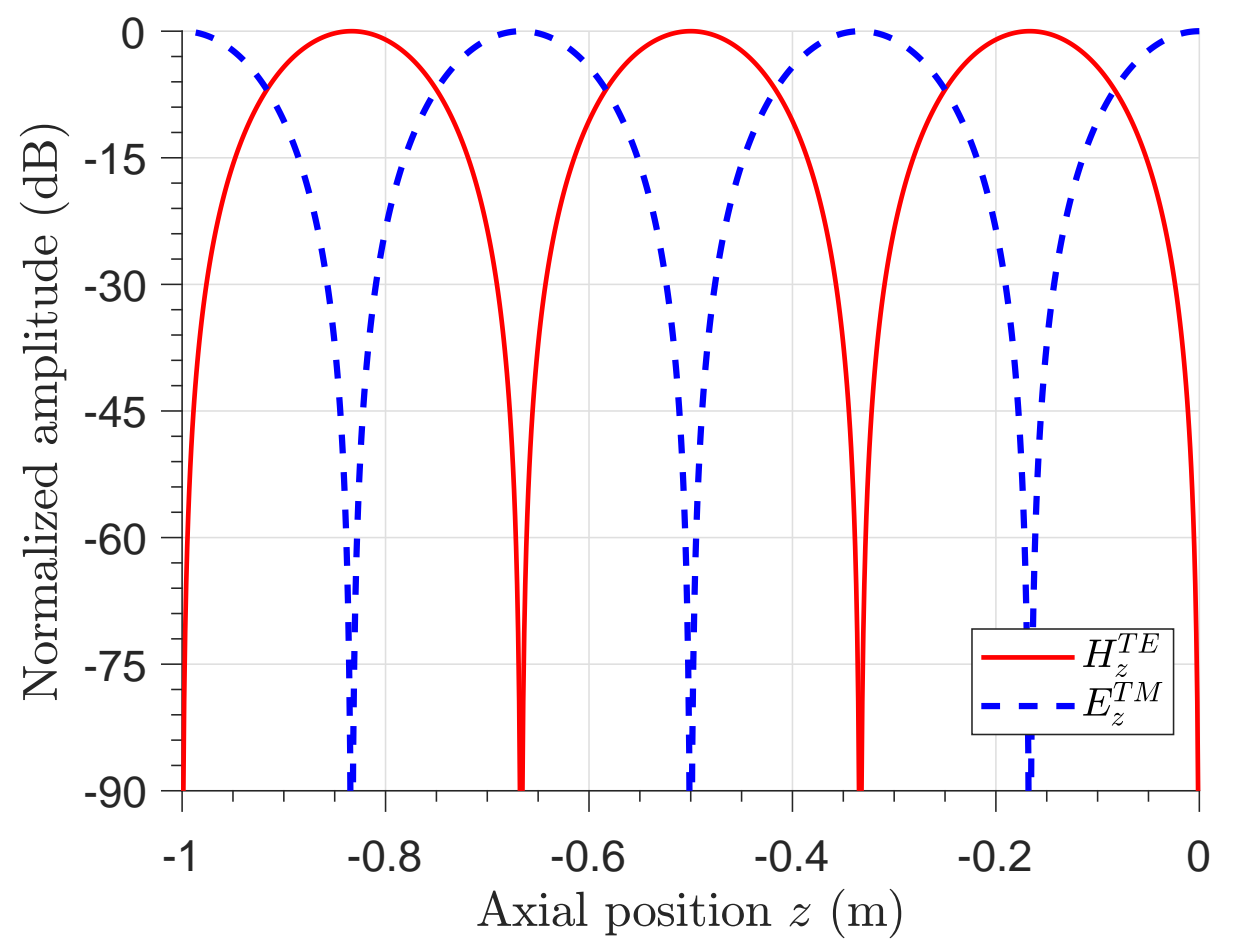

Figure 4.21: Normalized amplitude of the axial component of the electric and magnetic field $\left(E_{z}\right.$ and $H_{z}$ ) in a homogeneous and isotropic medium confined by a parallel plate waveguide (Third Mode). 


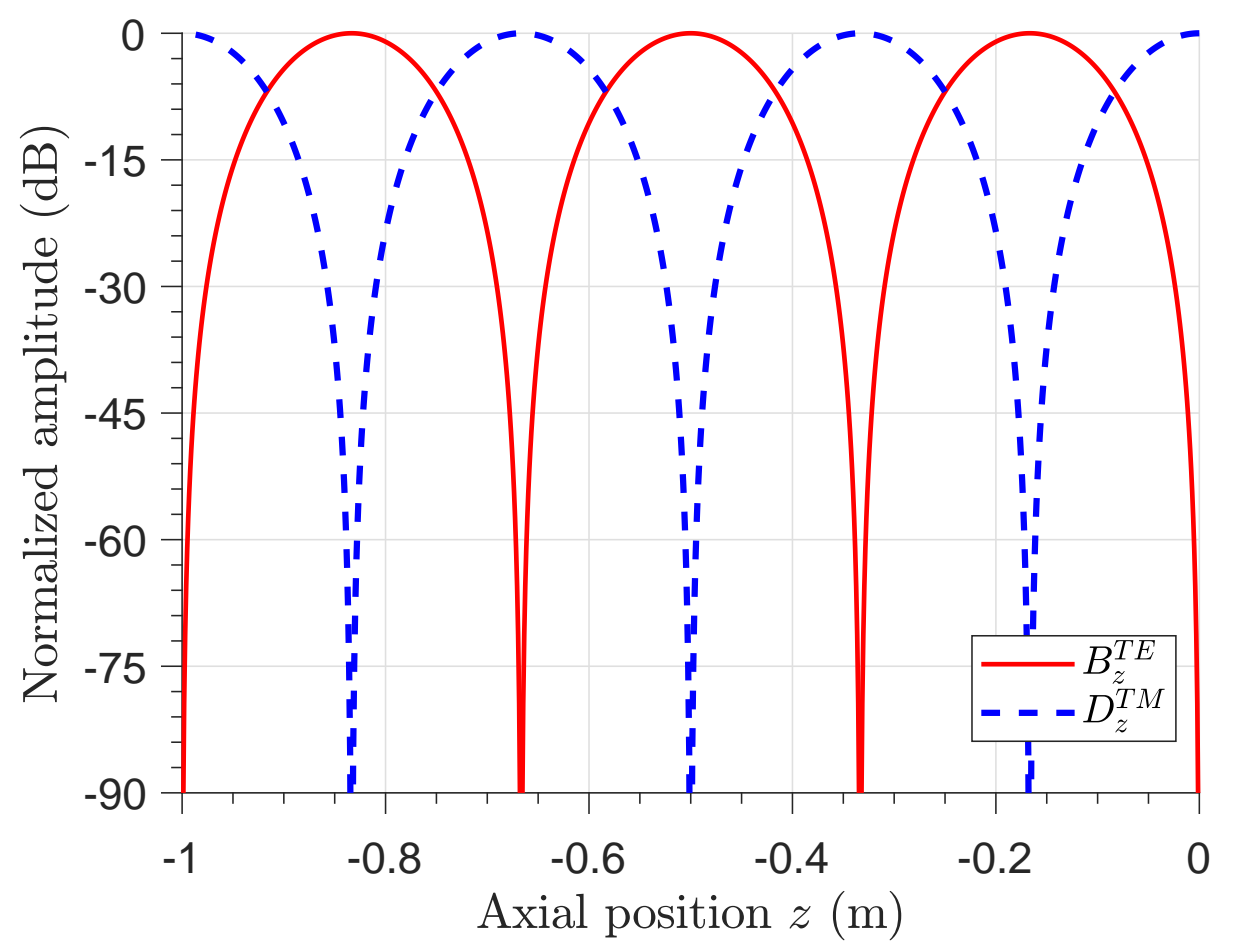

Figure 4.22: Normalized amplitude of the electric flux density and magnetic flux density $\left(D_{z}\right.$ and $\left.B_{z}\right)$ in a homogeneous and isotropic medium confined by a parallel plate waveguide (Third Mode).

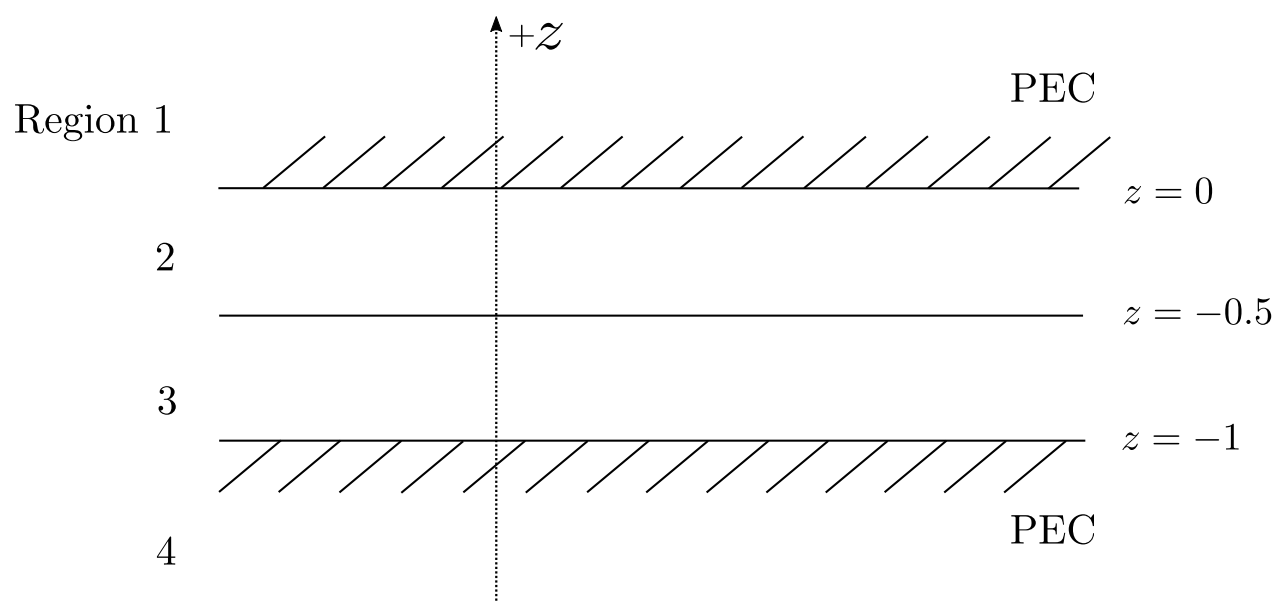

Figure 4.23: Axially-stratified two-layers geophysical formation. 


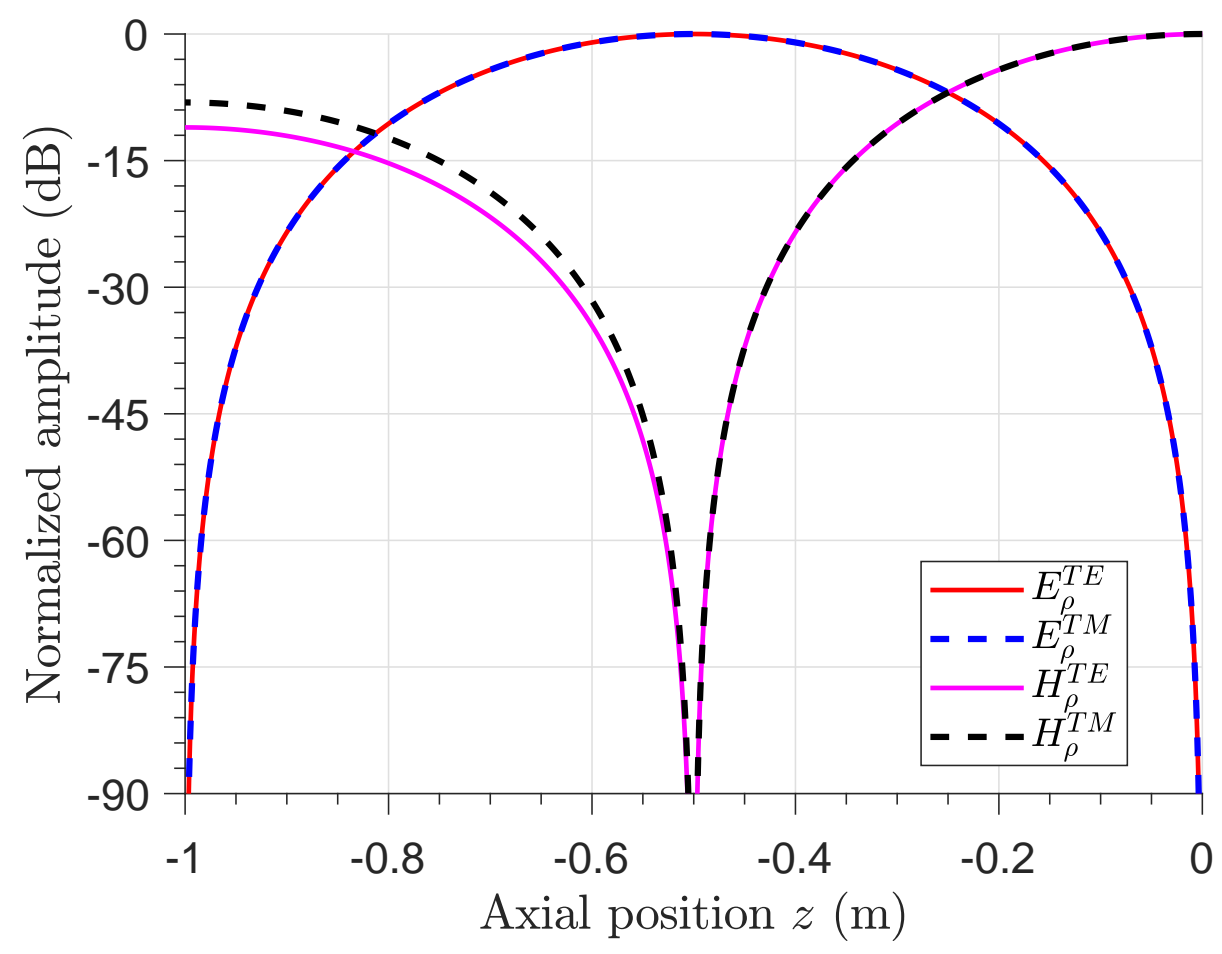

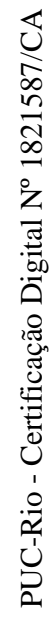

Figure 4.24: Normalized amplitude of the radial component of the electric and magnetic field $\left(E_{\rho}\right.$ and $\left.H_{\rho}\right)$ for decoupled TE and TM modes in an axiallystratified two-layers isotropic medium (First Mode).

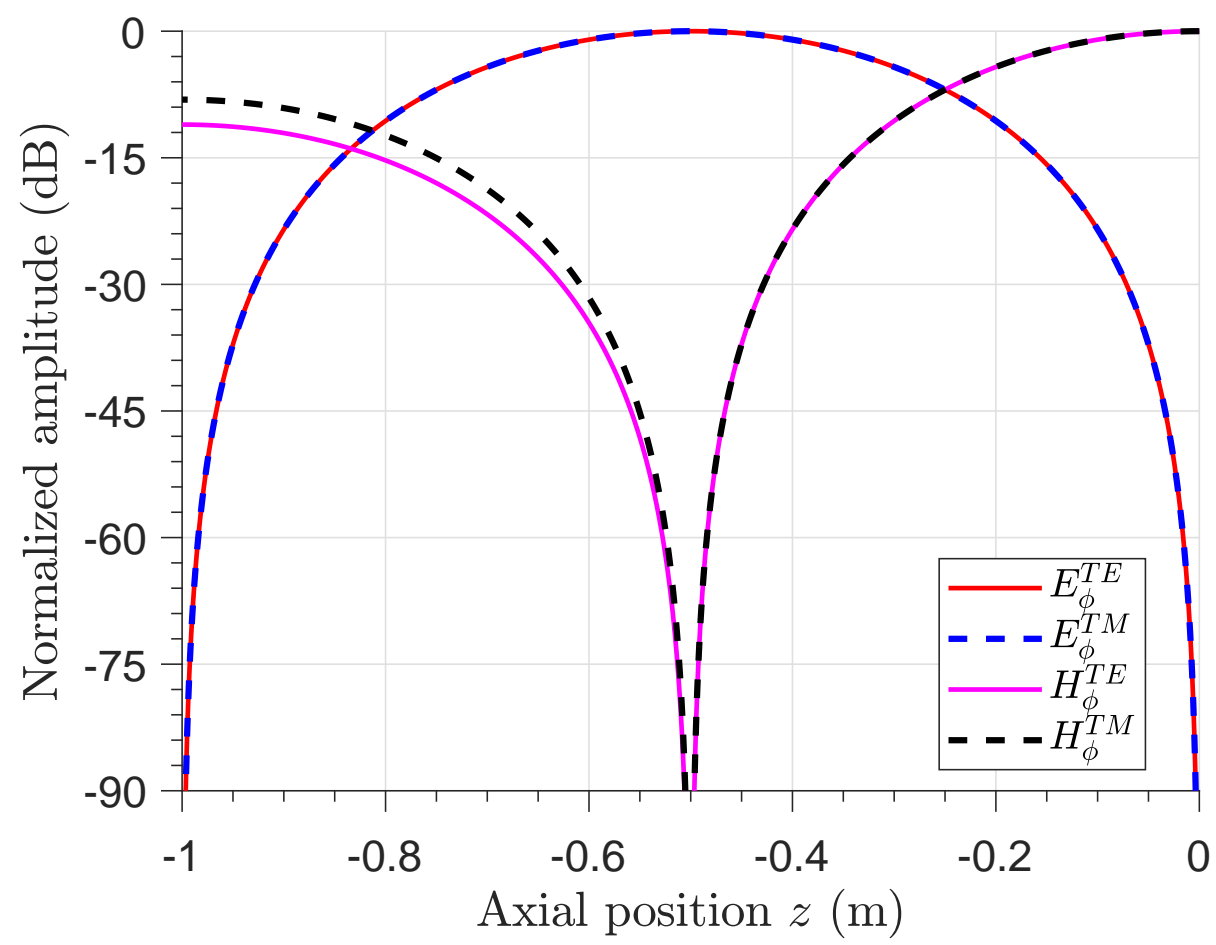

Figure 4.25: Normalized amplitude of the azimuthal component of the electric and magnetic field $\left(E_{\phi}\right.$ and $\left.H_{\phi}\right)$ for decoupled TE and TM modes in an axiallystratified two-layers isotropic medium (First Mode). 


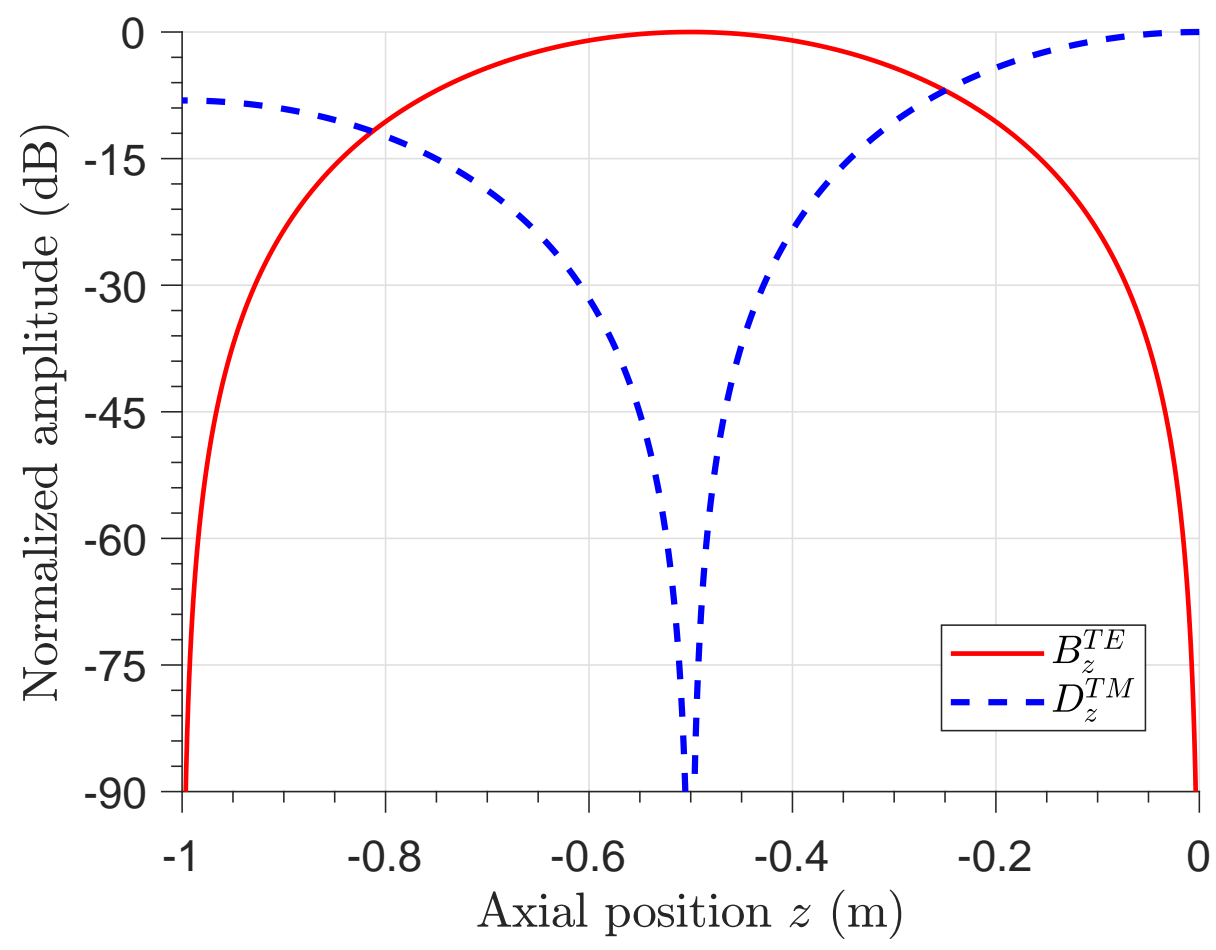

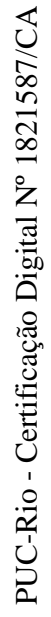

Figure 4.26: Normalized amplitude of the electric flux density and magnetic flux density $\left(D_{z}\right.$ and $\left.B_{z}\right)$ in axially-stratified two-layers isotropic medium (First Mode).

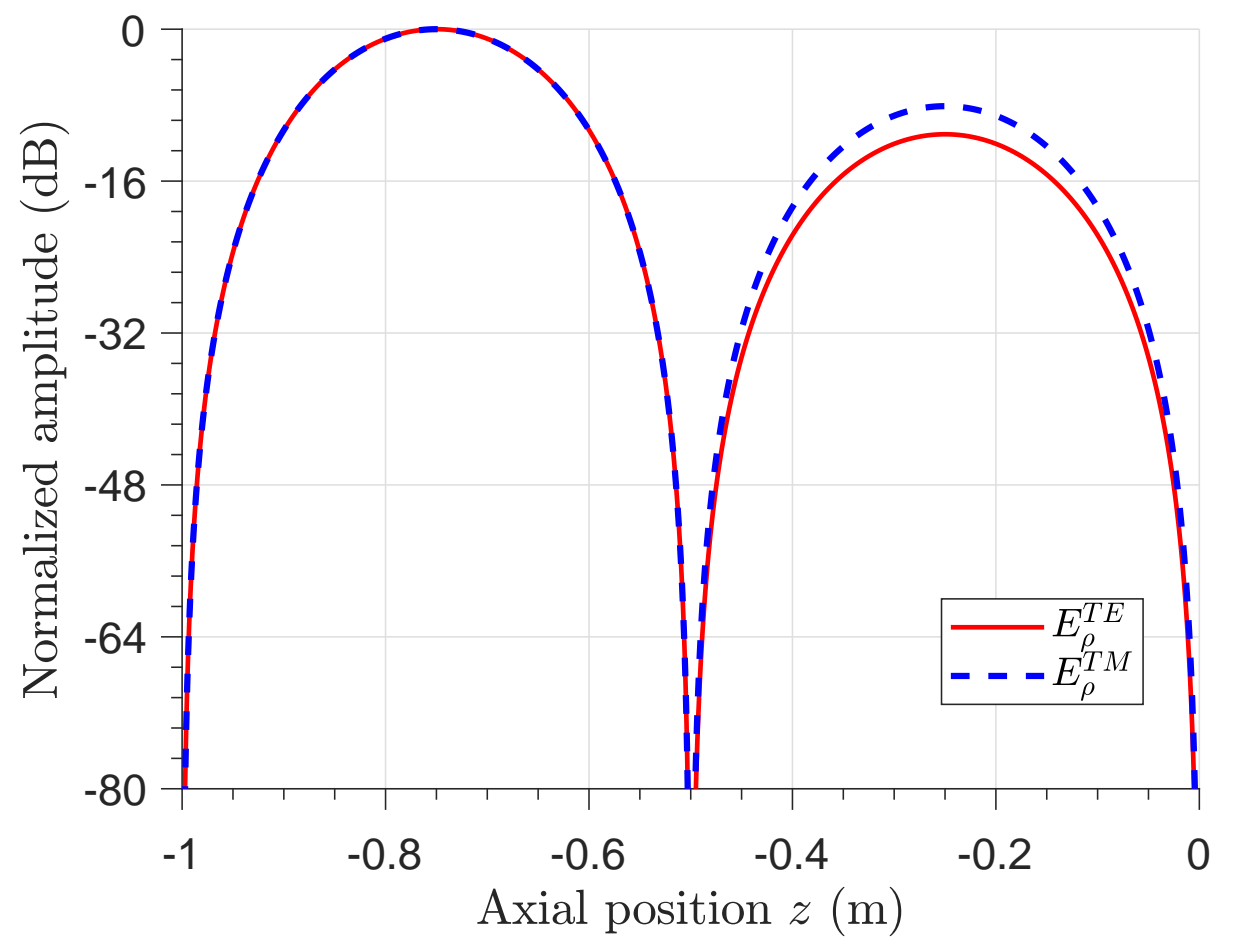

Figure 4.27: Normalized amplitude of the radial component of the electric field $\left(E_{\rho}\right)$ for decoupled TE and TM modes in an axially-stratified two-layers isotropic medium (Second Mode). 


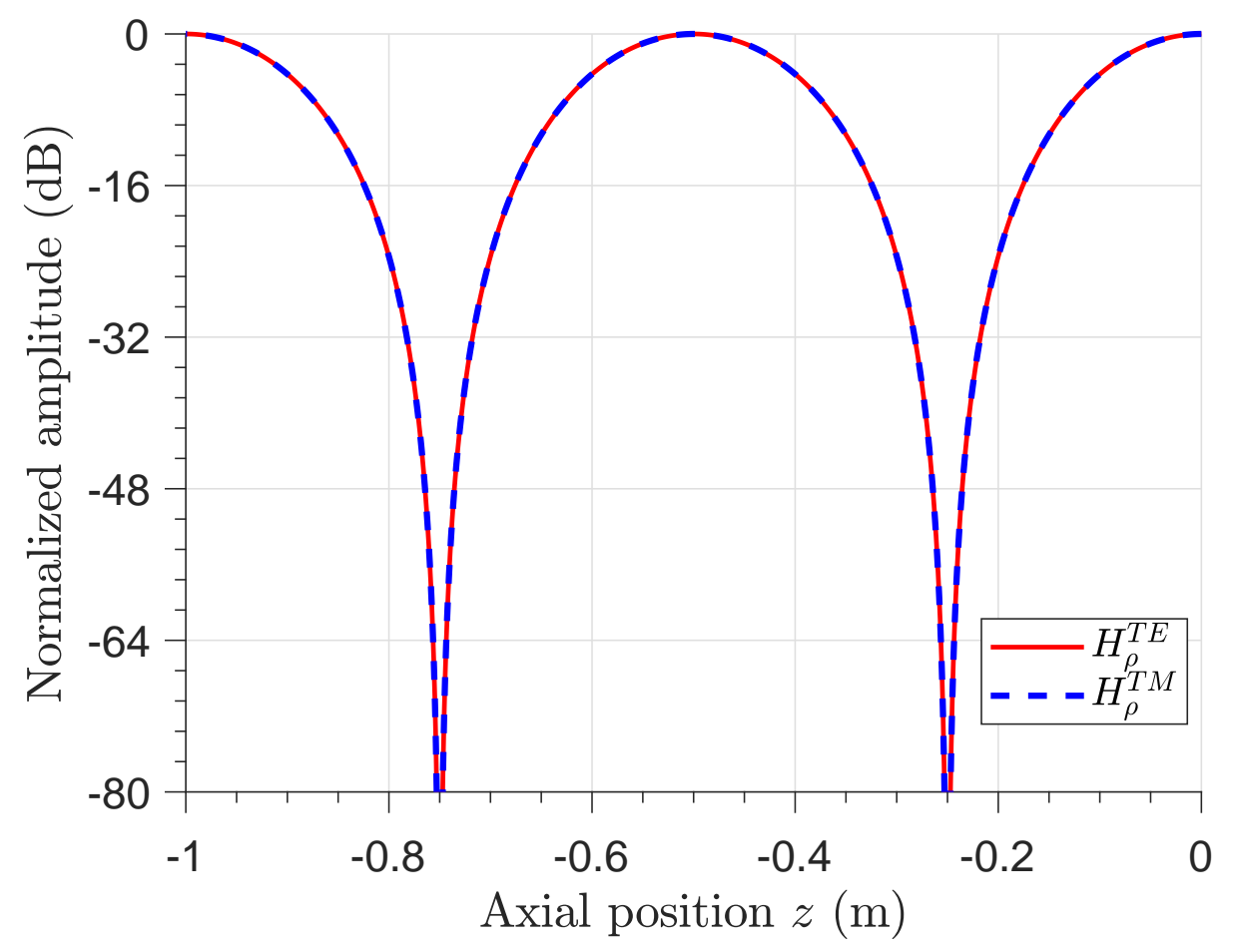

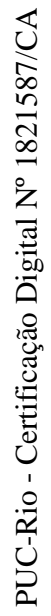

Figure 4.28: Normalized amplitude of the radial component of the magnetic field $\left(H_{\rho}\right)$ for decoupled TE and TM modes in an axially-stratified two-layers isotropic medium (Second Mode).

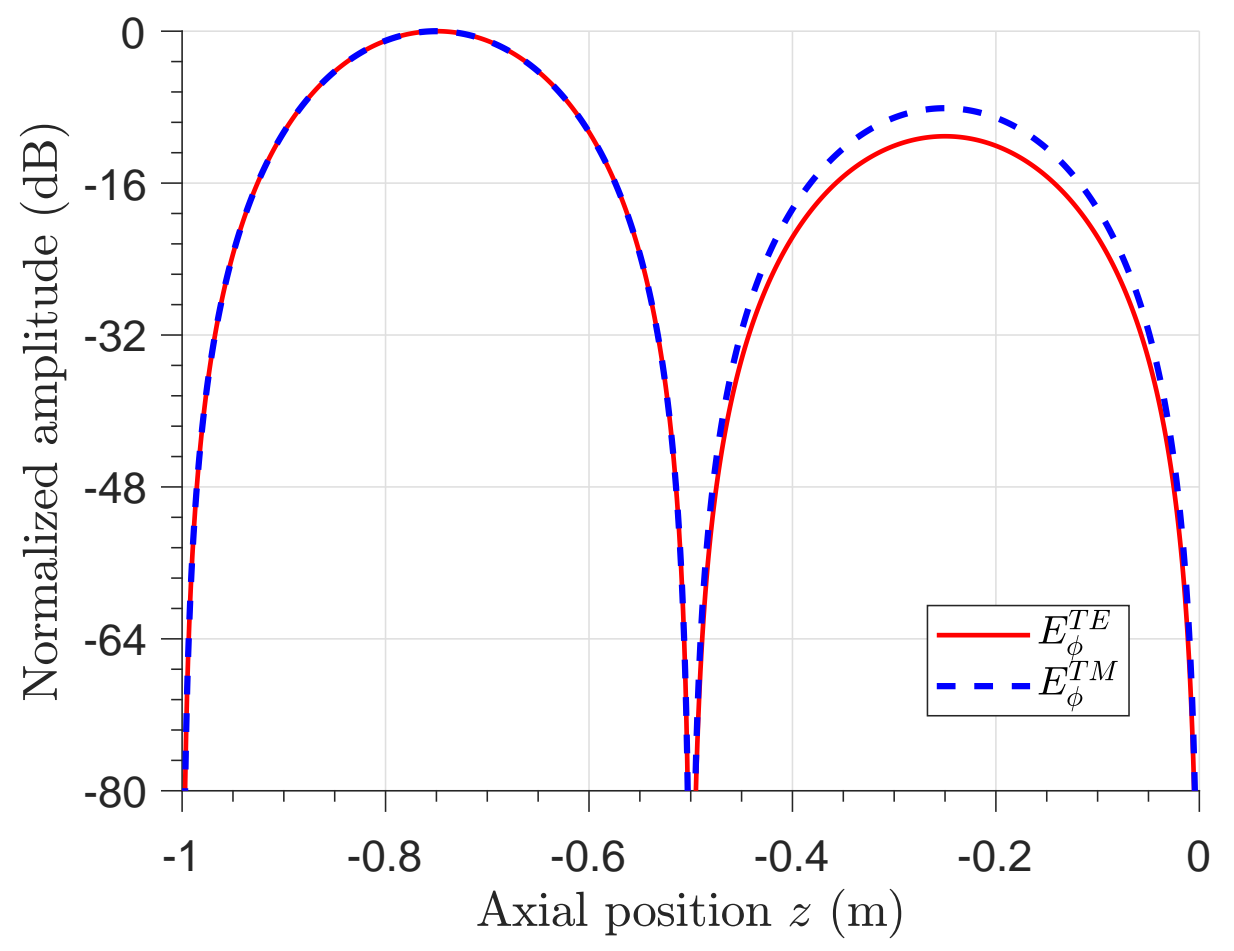

Figure 4.29: Normalized amplitude of the azimuthal component of the electric field $\left(E_{\phi}\right)$ for decoupled TE and TM modes in an axially-stratified two-layers isotropic medium (Second Mode). 


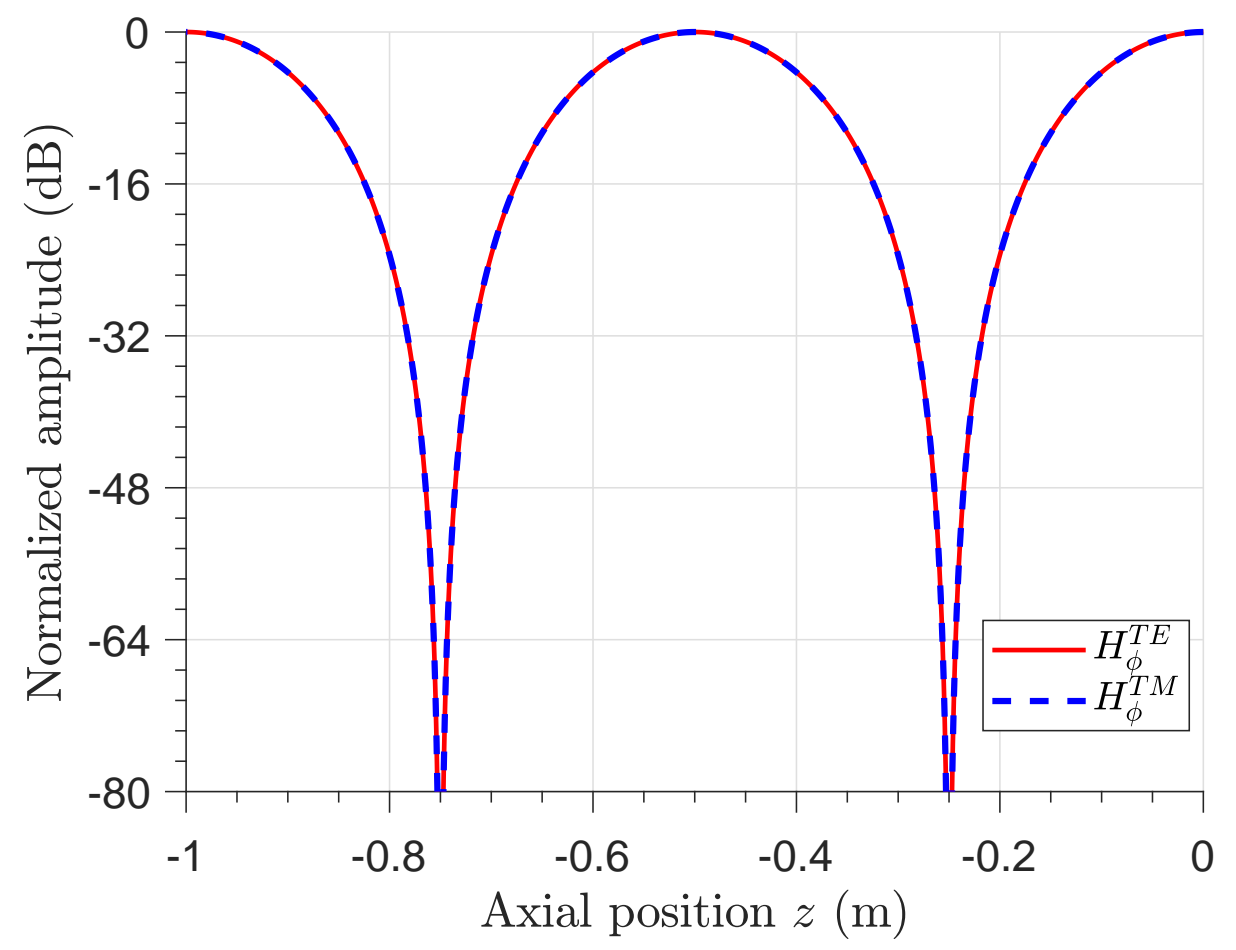

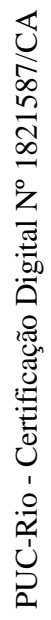

Figure 4.30: Normalized amplitude of the azimuthal component of the magnetic field $\left(H_{\phi}\right)$ for decoupled TE and TM modes in an axially-stratified two-layers isotropic medium (Second Mode).

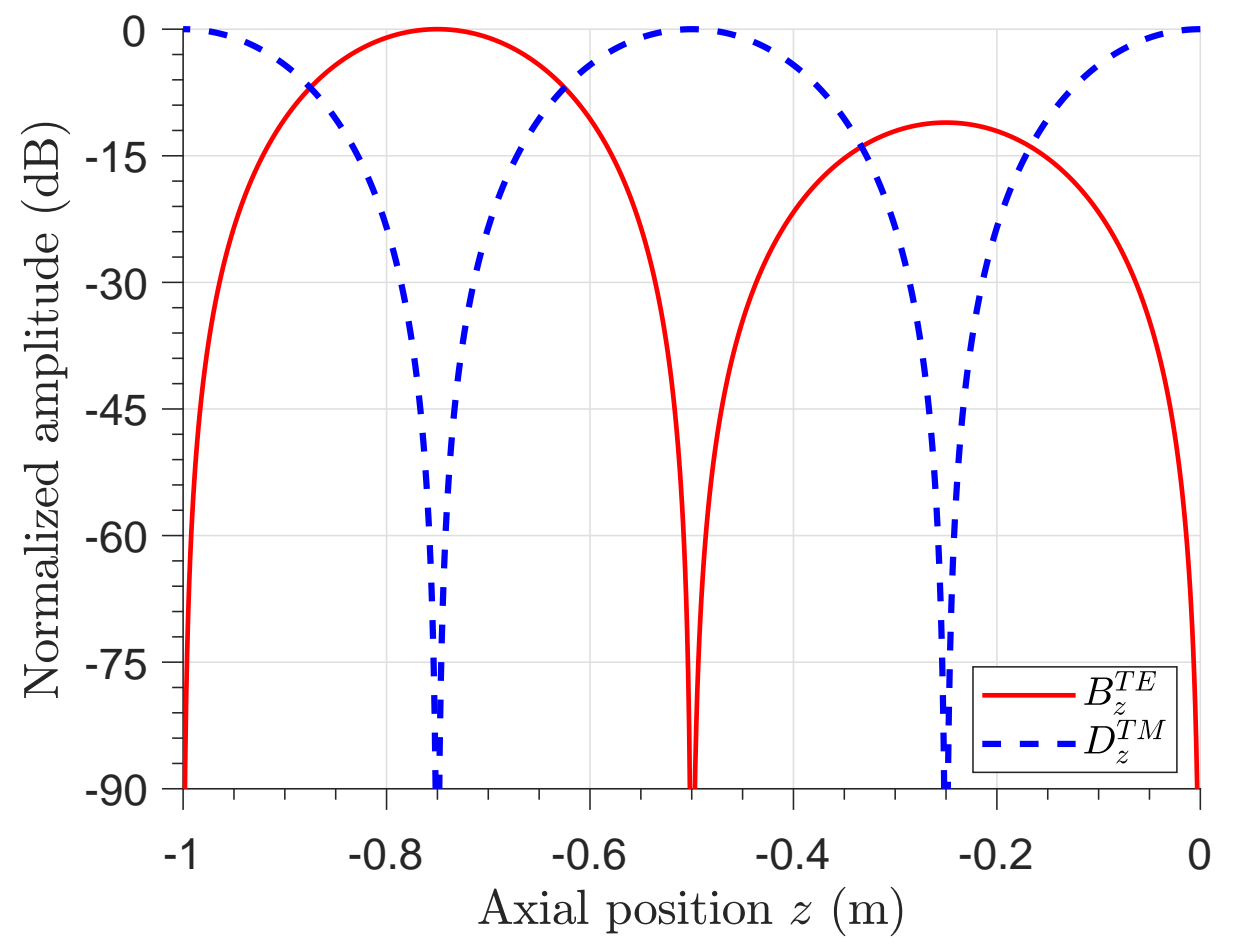

Figure 4.31: Normalized amplitude of the electric flux density and magnetic flux density $\left(D_{z}\right.$ and $\left.B_{z}\right)$ in axially-stratified two-layers isotropic medium (Second Mode). 


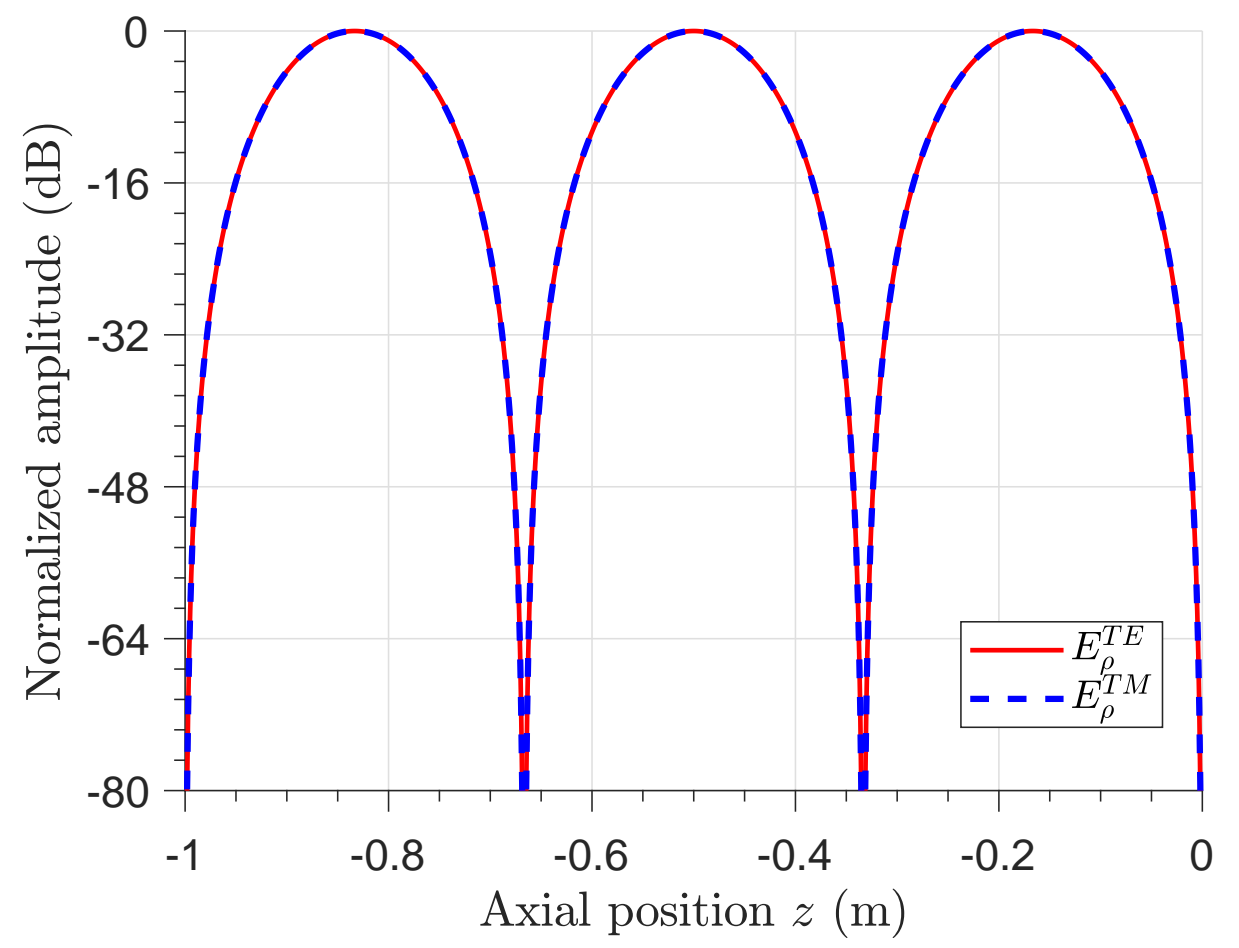

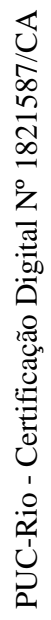

Figure 4.32: Normalized amplitude of the radial component of the electric field $\left(E_{\rho}\right)$ for decoupled TE and TM modes in an axially-stratified two-layers isotropic medium (Third Mode).

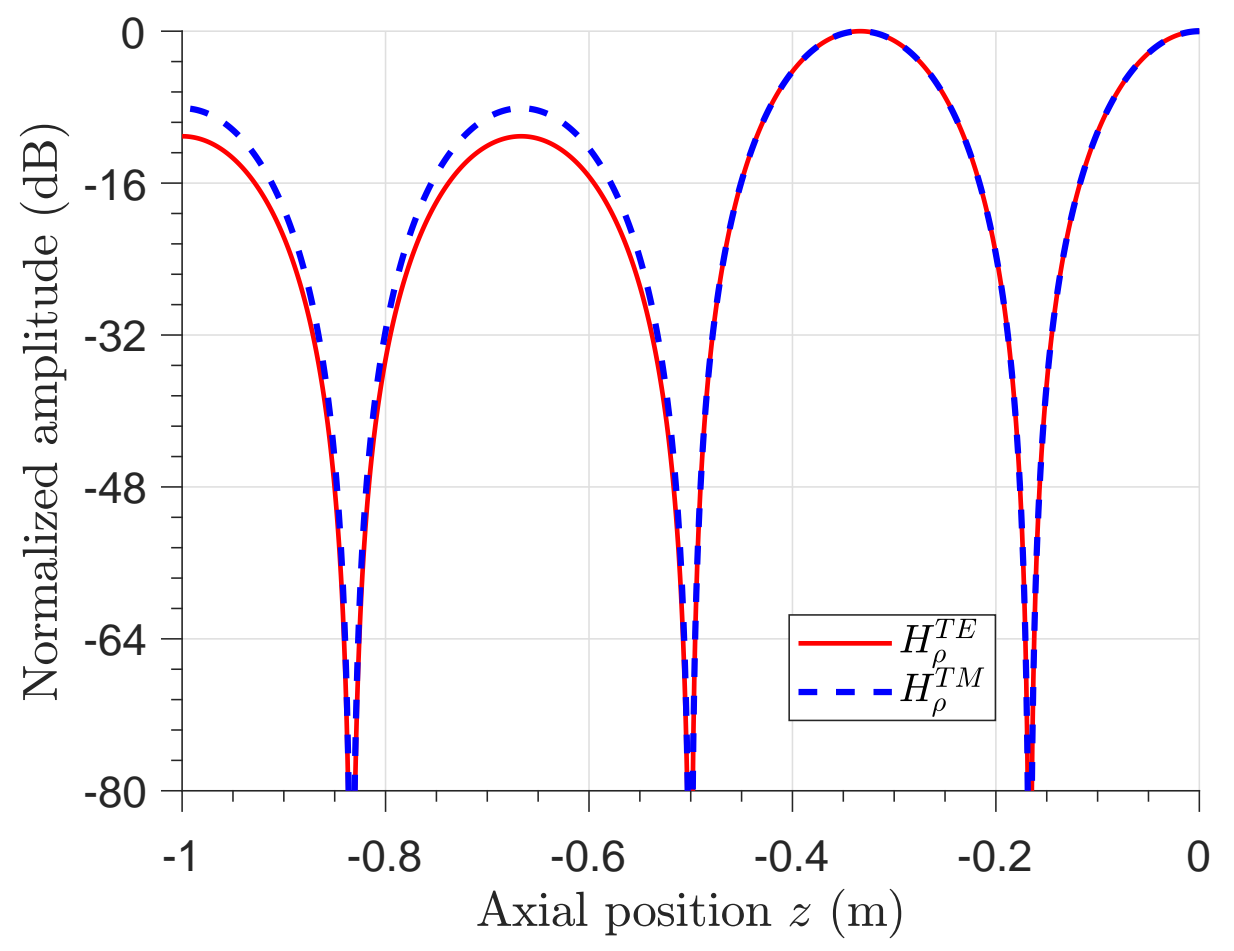

Figure 4.33: Normalized amplitude of the radial component of the magnetic field $\left(H_{\rho}\right)$ for decoupled TE and TM modes in an axially-stratified two-layers isotropic medium (Third Mode). 


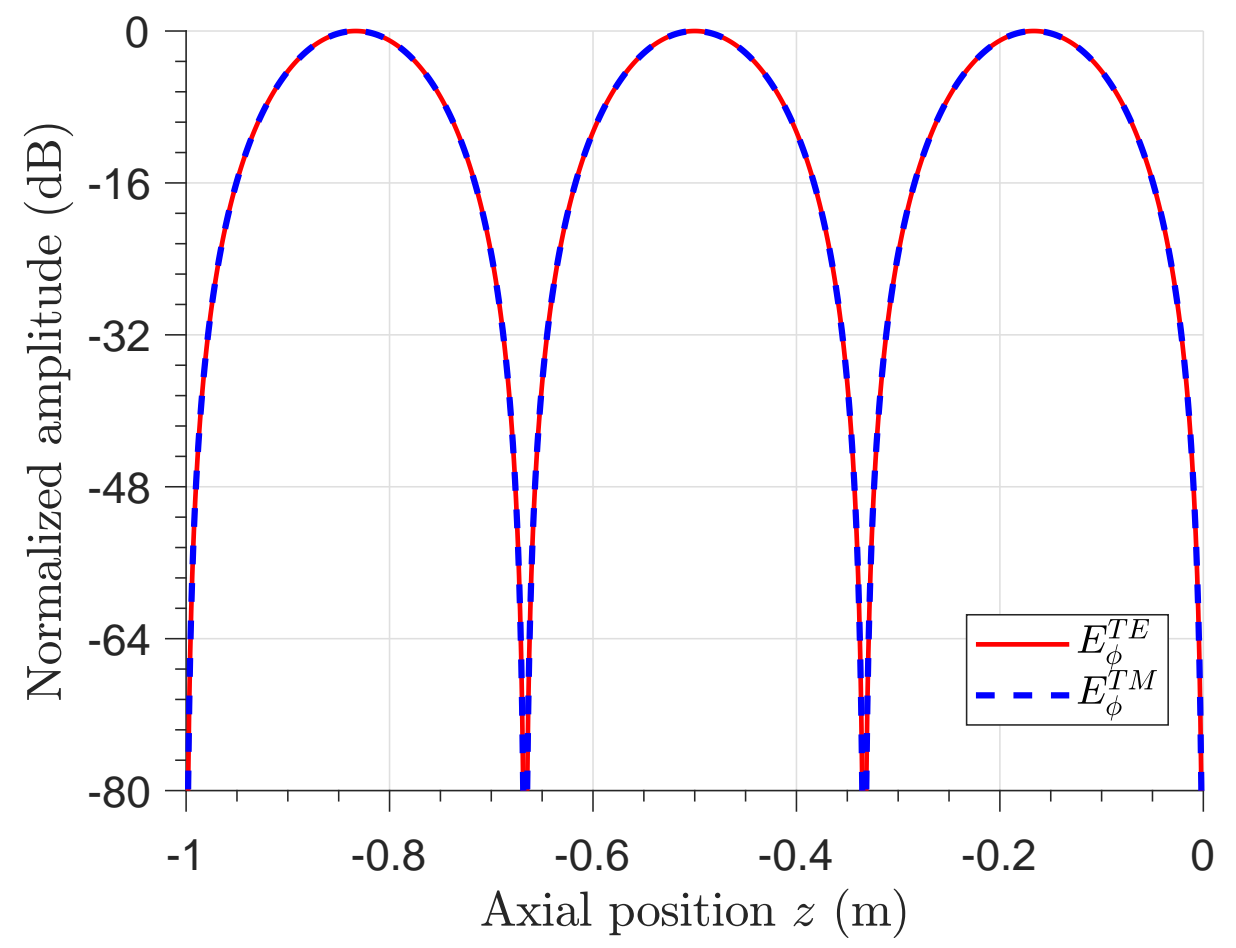

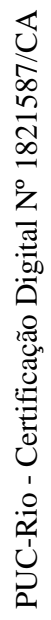

Figure 4.34: Normalized amplitude of the azimuthal component of the electric field $\left(E_{\phi}\right)$ for decoupled TE and TM modes in an axially-stratified two-layers isotropic medium (Third Mode).

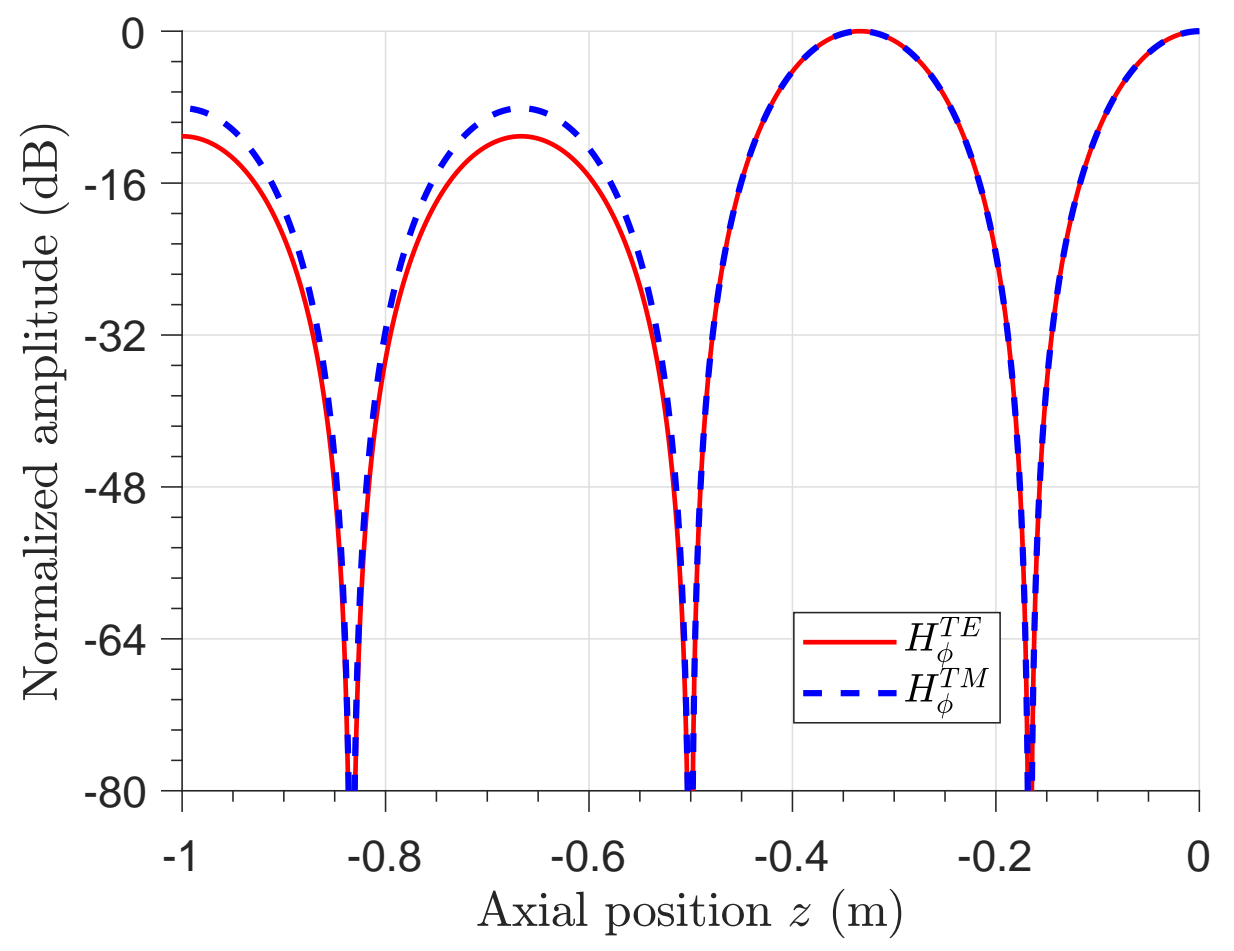

Figure 4.35: Normalized amplitude of the azimuthal component of the magnetic field $\left(H_{\phi}\right)$ for decoupled TE and TM modes in an axially-stratified two-layers isotropic medium (Third Mode). 


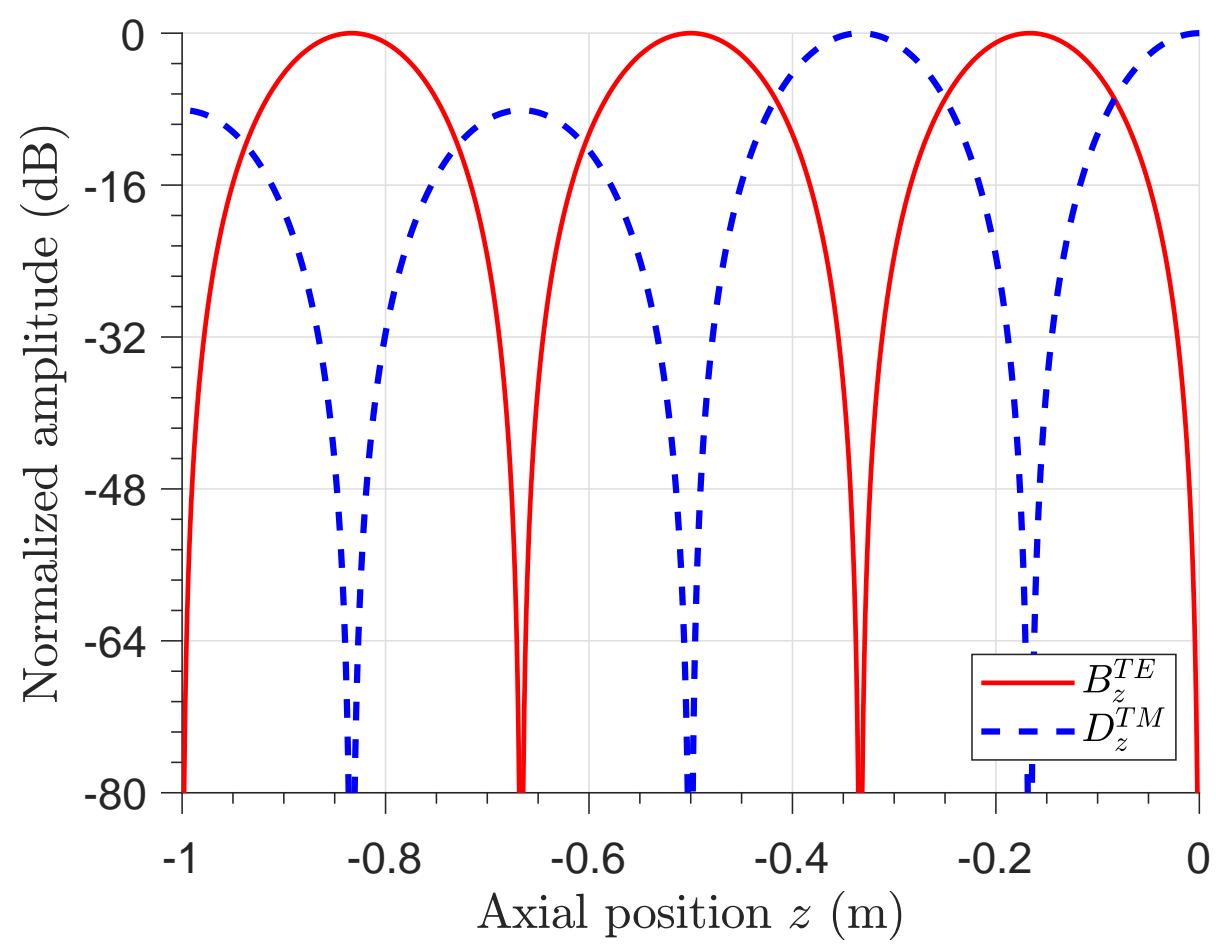

Figure 4.36: Normalized amplitude of the electric flux density and magnetic flux density $\left(D_{z}\right.$ and $\left.B_{z}\right)$ in axially-stratified two-layers isotropic medium (Third Mode).

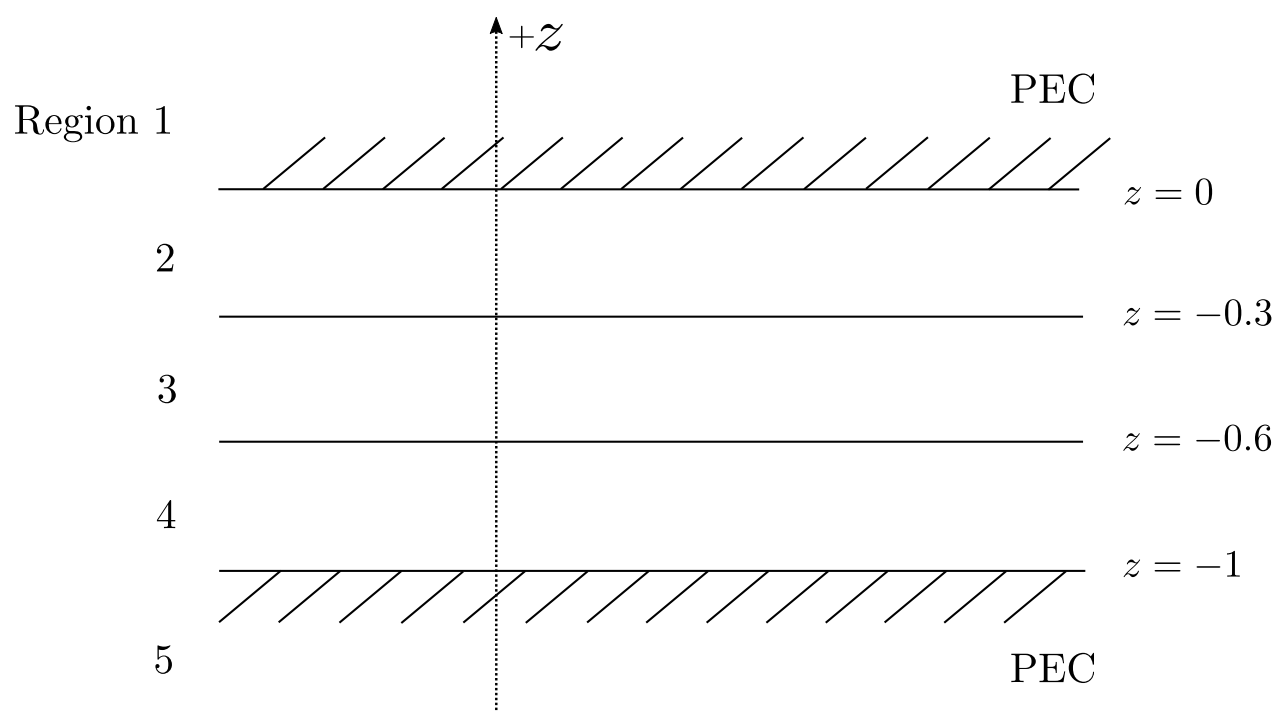

Figure 4.37: Axially-stratified three-layers geophysical formation. 


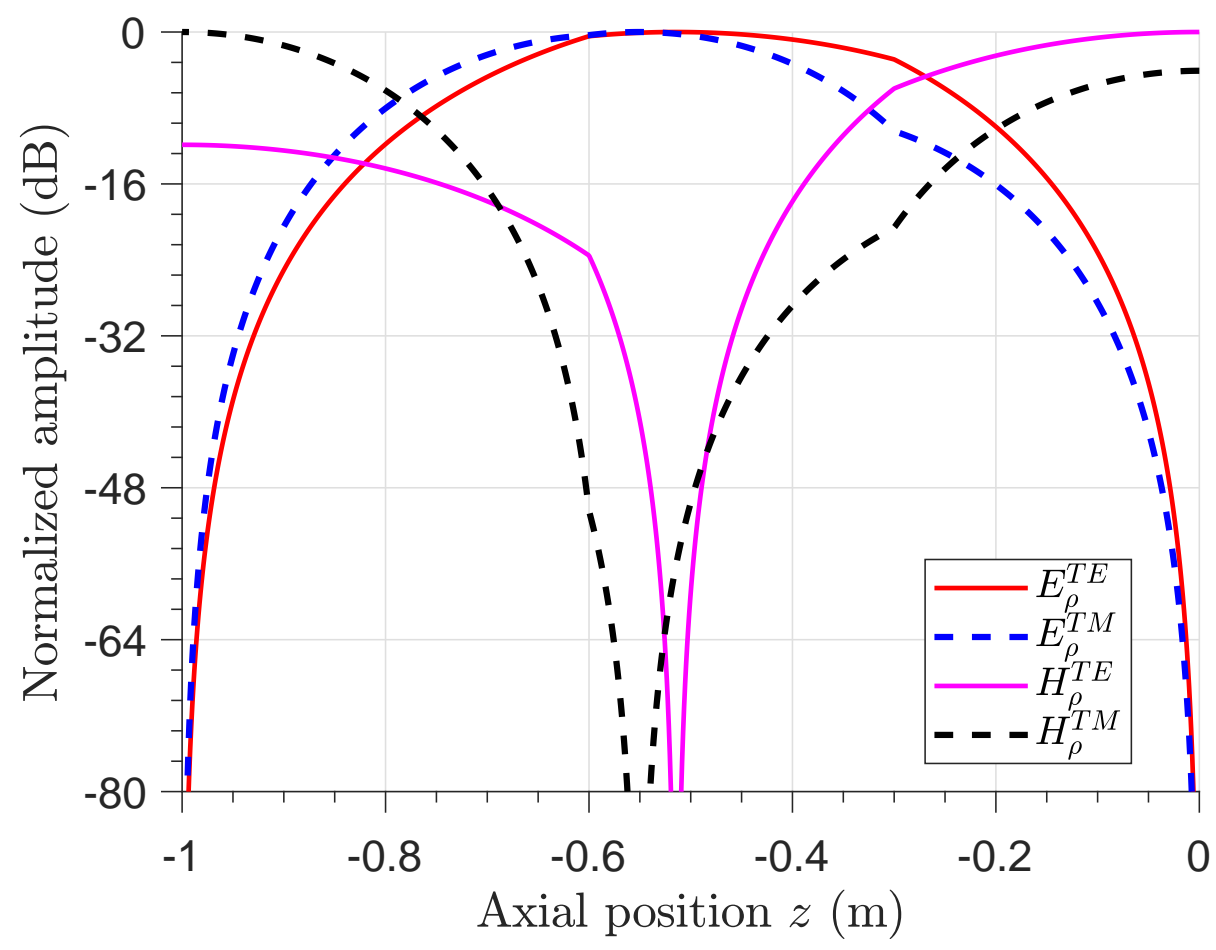

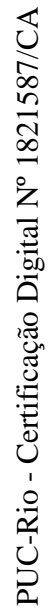

Figure 4.38: Normalized amplitude of the radial component of the electric and magnetic field $\left(E_{\rho}\right.$ and $\left.H_{\rho}\right)$ for decoupled TE and TM modes in an axiallystratified three-layers isotropic medium (First Mode).

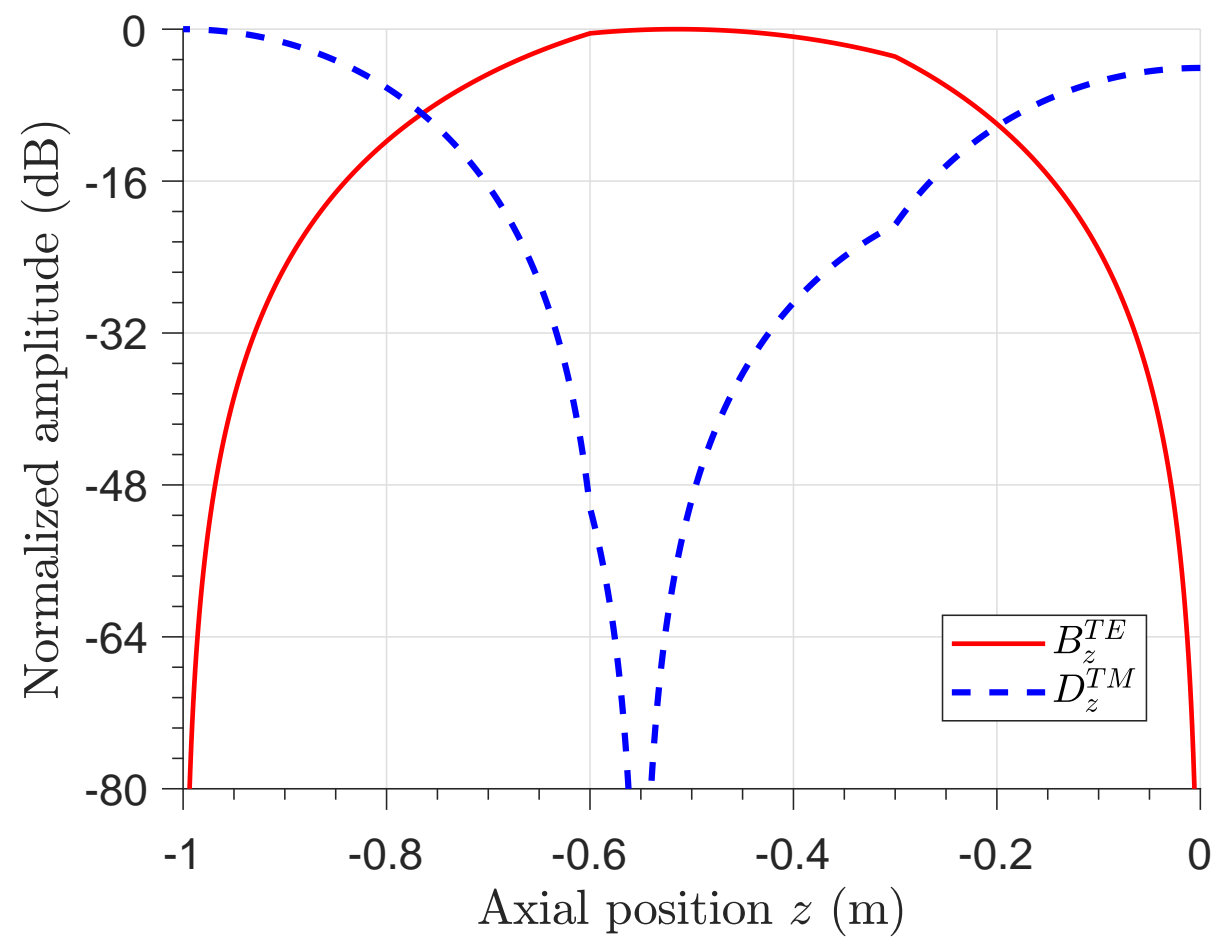

Figure 4.39: Normalized amplitude of the electric flux density and magnetic flux density $\left(D_{z}\right.$ and $\left.B_{z}\right)$ in axially-stratified three-layers isotropic medium (First Mode). 


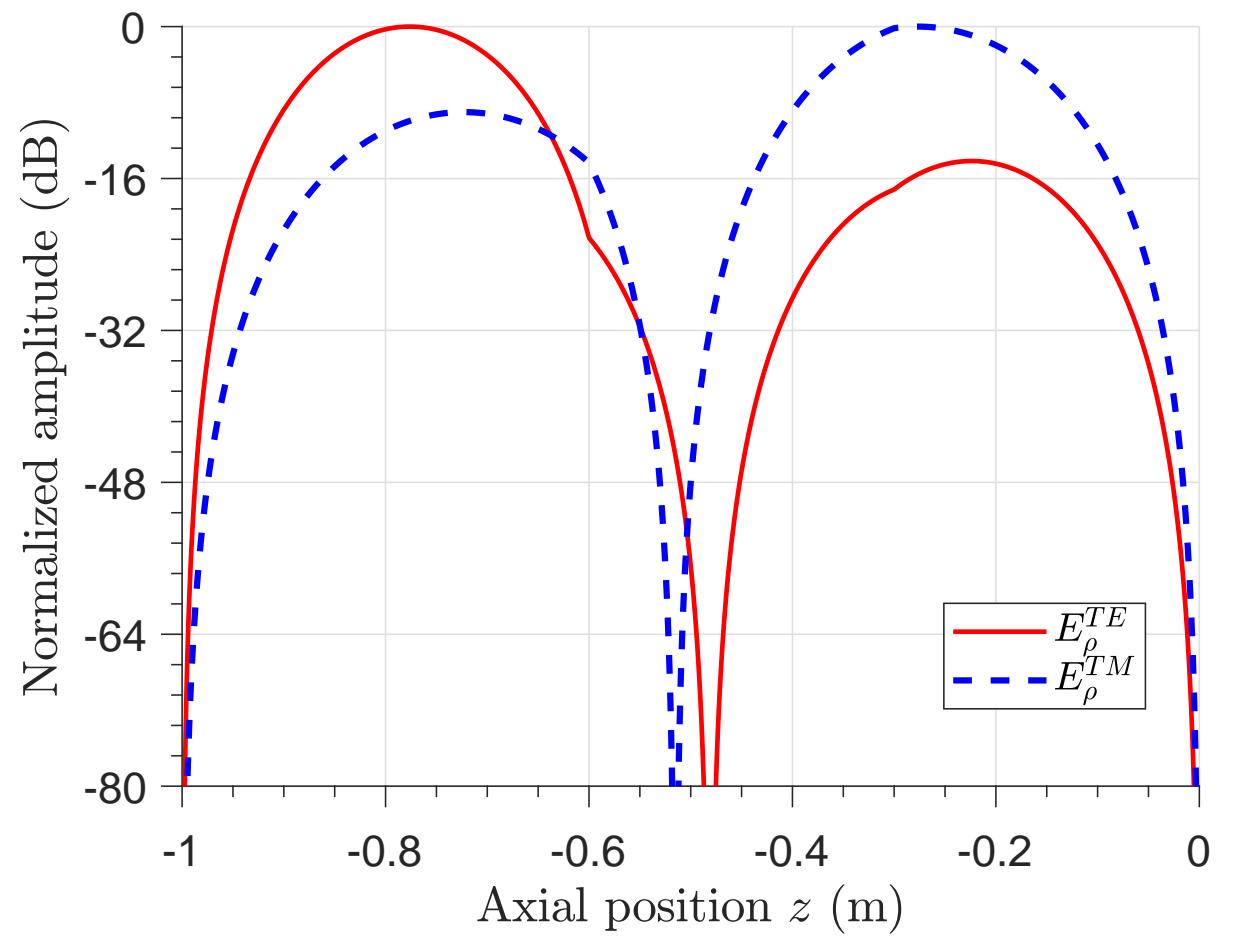

Figure 4.40: Normalized amplitude of the radial component of the electric field $\left(E_{\rho}\right)$ for decoupled TE and TM modes in an axially-stratified three-layers isotropic medium (Second Mode). 


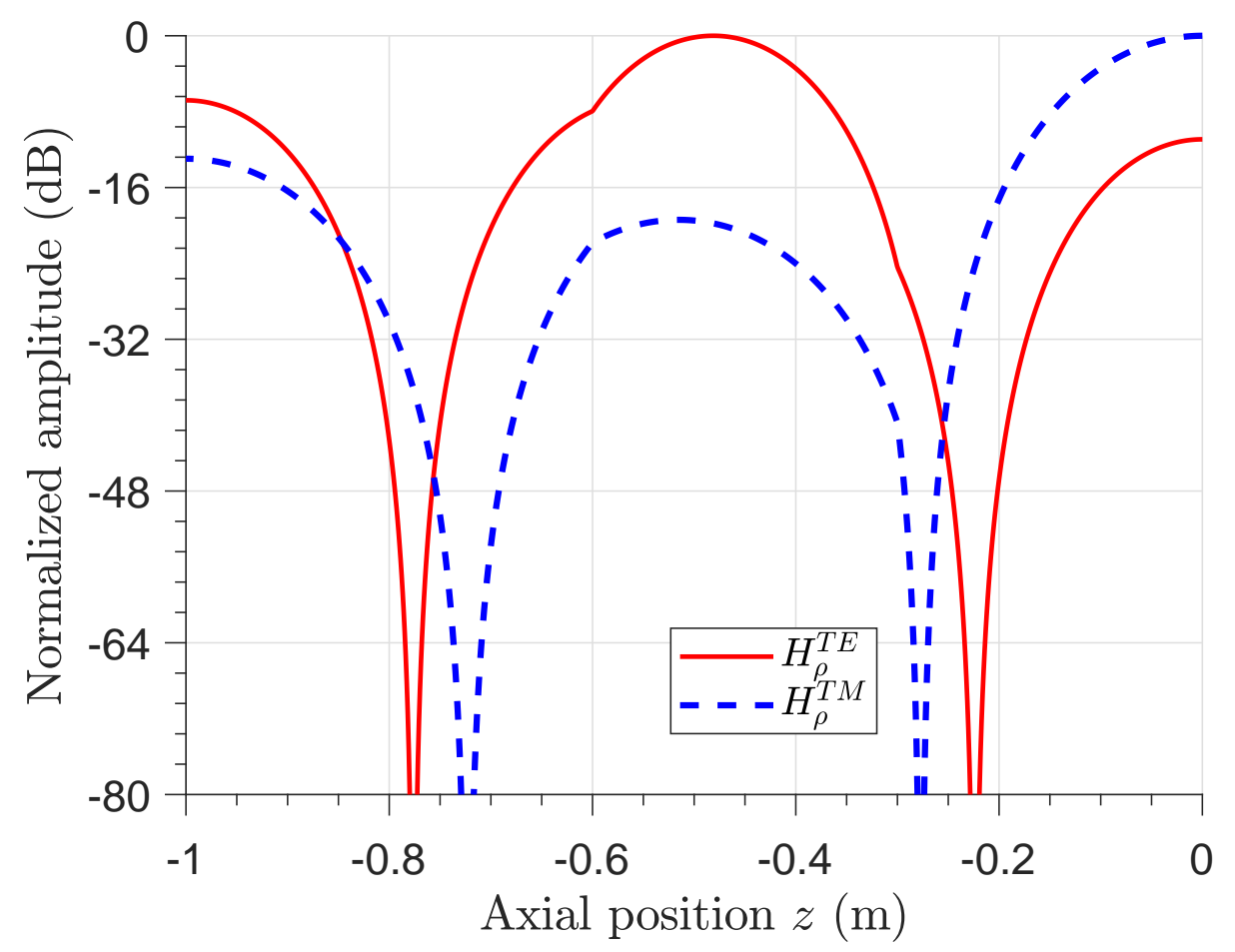

Figure 4.41: Normalized amplitude of the radial component of the magnetic field $\left(H_{\rho}\right)$ for decoupled TE and TM modes in an axially-stratified three-layers isotropic medium (Second Mode).

In this case we modeled with more difference in the physic characteristics between the layers of formation than in the previous examples. Therefore, by having more variability in its behavior allows us to notice the continuity of both fields across of each interface of medium are also satisfied.

\section{Four-Layer Isotropic Formation}

In this case is considered a horizontally stratified formation with four layers in the $\hat{z}$-position $z=0, z=-0.2, z=-0.5, z=-0.8$ and $z=-1 \mathrm{~m}$. The geometry of problem is analogous to all previous ones, except in number and position of interfaces already mentioned above. The physical characteristics and resulting eigenvalues for this example are presented in Table 4.6 and 4.7, respectively. The propagation sense of the transmitted waves is in the negative direction of the axial domain. In order to avoid repetition in relation to the previous examples, only the electric and magnetic flux densities are represented for the first three modes from Fig. 4.46 to Fig. 4.48.

\section{Five-Layer Isotropic Formation}

For ensuring the correct functioning of the implemented method for isotropic media, we validate a last example for this type of formations. Now we 


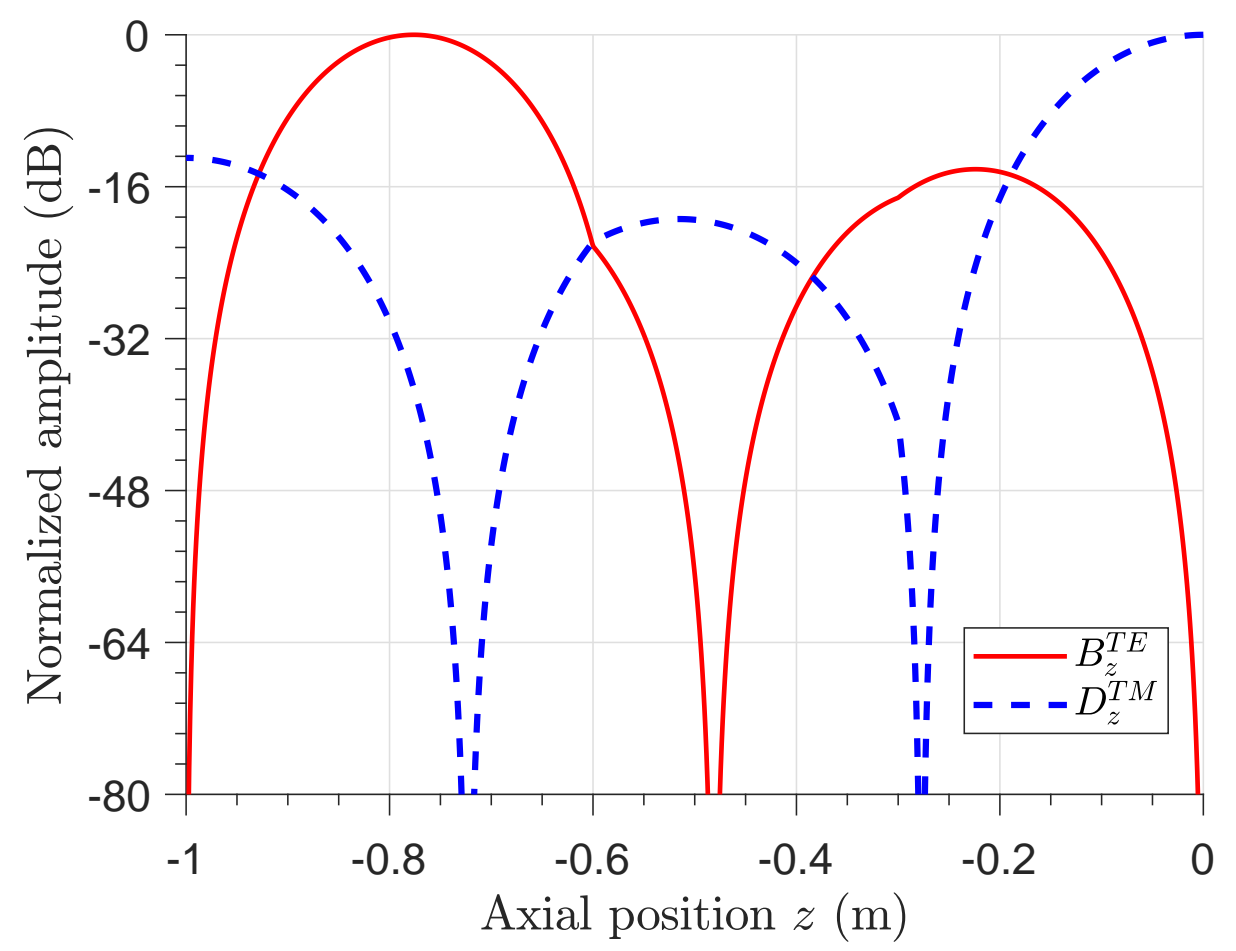

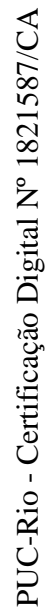

Figure 4.42: Normalized amplitude of the electric flux density and magnetic flux density $\left(D_{z}\right.$ and $\left.B_{z}\right)$ in axially-stratified three-layers isotropic medium (Second Mode).

Table 4.6: Physical characteristics of an axially-stratified four-layers isotropic medium.

\begin{tabular}{c|c|c}
\hline \hline Layer & Relative Magnetic Permeability & Relative Electric Permittivity \\
\hline \hline 2 & $\mu_{r\{s, z\}}=3.8$ & $\epsilon_{r\{s, z\}}=4.5$ \\
\hline 3 & $\mu_{r\{s, z\}}=2.1$ & $\epsilon_{r\{s, z\}}=2.4$ \\
\hline 4 & $\mu_{r\{s, z\}}=1.3$ & $\epsilon_{r\{s, z\}}=1.7$ \\
\hline 5 & $\mu_{r\{s, z\}}=1.5$ & $\epsilon_{r\{s, z\}}=1.2$ \\
\hline \hline
\end{tabular}

Table 4.7: Eigenvalues for an axially-stratified four-layers isotropic medium.

\begin{tabular}{c|c|c}
\hline \hline \multirow{2}{*}{ Mode Number } & \multicolumn{2}{|c}{ Eigenvalue $\boldsymbol{k}_{\boldsymbol{\rho}, \boldsymbol{n} \boldsymbol{p}}$} \\
\cline { 2 - 3 } & TE & TM \\
\hline \hline 1st. & $2.837777570398038 \mathrm{i}$ & $3.309772026956289 \mathrm{i}$ \\
\hline 2nd. & $6.058185563297657 \mathrm{i}$ & $6.371034773831773 \mathrm{i}$ \\
\hline 3rd. & $9.593169665715536 \mathrm{i}$ & $9.241600211295243 \mathrm{i}$ \\
\hline \hline
\end{tabular}

consider an axially-stratified five-layers gephysical formation with interfaces of $\hat{z}$-position $z=-0.2, z=-0.4, z=-0.6, z=-0.8$ and $z=-1$. The physical 


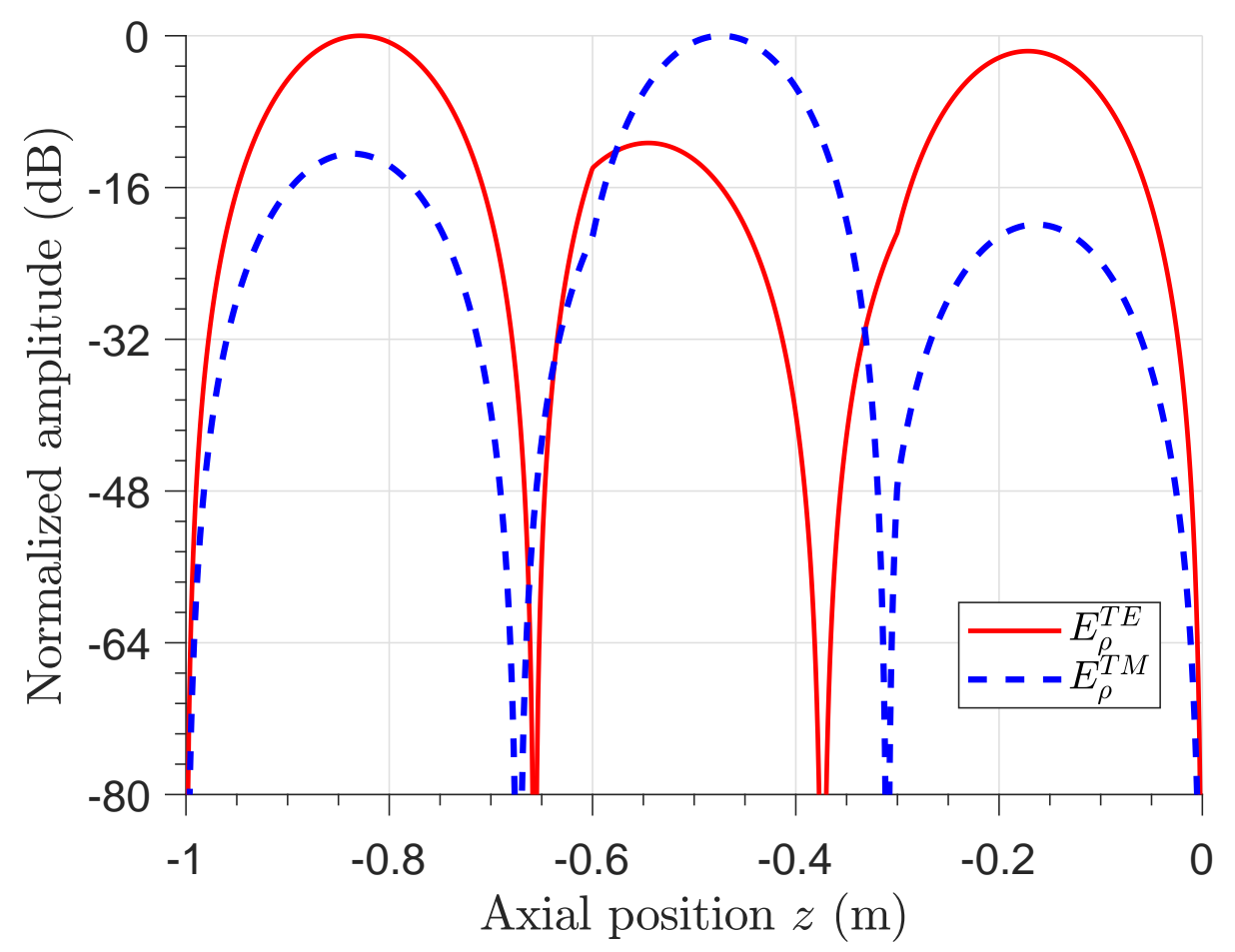

Figure 4.43: Normalized amplitude of the radial component of the electric field $\left(E_{\rho}\right)$ for decoupled TE and TM modes in an axially-stratified three-layers isotropic medium (Third Mode).

characteristics of medium and eigenvalues for this scenario are showed in Table 4.8 and 4.9, respectively. The propagation direction of the transmitted waves

Table 4.8: Physical characteristics of an axially-stratified five-layers isotropic medium.

\begin{tabular}{c|c|c}
\hline \hline Layer & Relative Magnetic Permeability & Relative Electric Permittivity \\
\hline \hline 2 & $\mu_{r\{s, z\}}=1.1$ & $\epsilon_{r\{s, z\}}=1.7$ \\
\hline 3 & $\mu_{r\{s, z\}}=2.3$ & $\epsilon_{r\{s, z\}}=2.9$ \\
\hline 4 & $\mu_{r\{s, z\}}=4.2$ & $\epsilon_{r\{s, z\}}=5.1$ \\
\hline 5 & $\mu_{r\{s, z\}}=2.0$ & $\epsilon_{r\{s, z\}}=2.5$ \\
\hline 6 & $\mu_{r\{s, z\}}=1.4$ & $\epsilon_{r\{s, z\}}=1.6$ \\
\hline \hline
\end{tabular}

is in the positive direction to $\hat{z}$. From Fig. 4.49 to Fig. 4.51 we show the electric and magnetic flux densities for the first three modes in Table 4.9. 


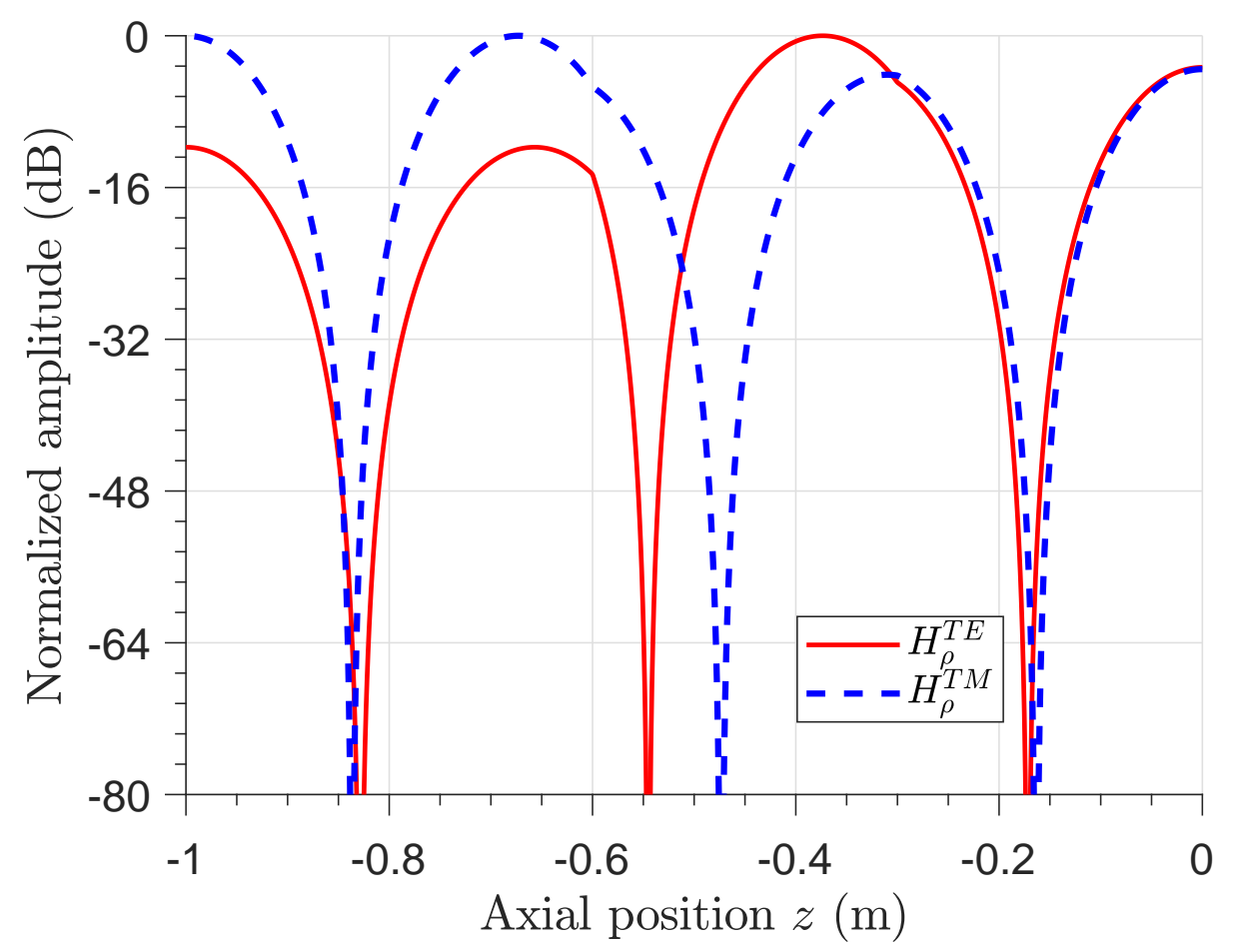

Figure 4.44: Normalized amplitude of the radial component of the magnetic field $\left(H_{\rho}\right)$ for decoupled TE and TM modes in an axially-stratified three-layers isotropic medium (Third Mode).

Table 4.9: Eigenvalues for an axially-stratified five-layers isotropic medium.

\begin{tabular}{c|c|c}
\hline \hline \multirow{2}{*}{ Mode Number } & \multicolumn{2}{|c}{ Eigenvalue $\boldsymbol{k}_{\boldsymbol{\rho}, \boldsymbol{n} \boldsymbol{p}}$} \\
\cline { 2 - 3 } & TE & TM \\
\hline \hline 1st. & $4.022658220958810 \mathrm{i}$ & $2.320863140103564 \mathrm{i}$ \\
\hline 2nd. & $6.075565230426880 \mathrm{i}$ & $6.645057418448241 \mathrm{i}$ \\
\hline 3rd. & $9.632383349494866 \mathrm{i}$ & $9.062887589461596 \mathrm{i}$ \\
\hline 4th. & $11.685281363592152 \mathrm{i}$ & $13.387081944397289 \mathrm{i}$ \\
\hline 5th. & $15.707957144302920 \mathrm{i}$ & $15.707960413373227 \mathrm{i}$ \\
\hline \hline
\end{tabular}

\subsubsection{4}

\section{Multi-Layer Anisotropic Formation}

In this section we consider a medium similar to the latter problem in the previous section, but with anisotropic formations. The physical characteristics and some resulting eigenvalues for this example are presented in Table 4.10 and 4.11, respectively. The electric and magnetic flux densities are represented from Fig. 4.52 to Fig. 4.54 for the first three modes in Table 4.11 . 


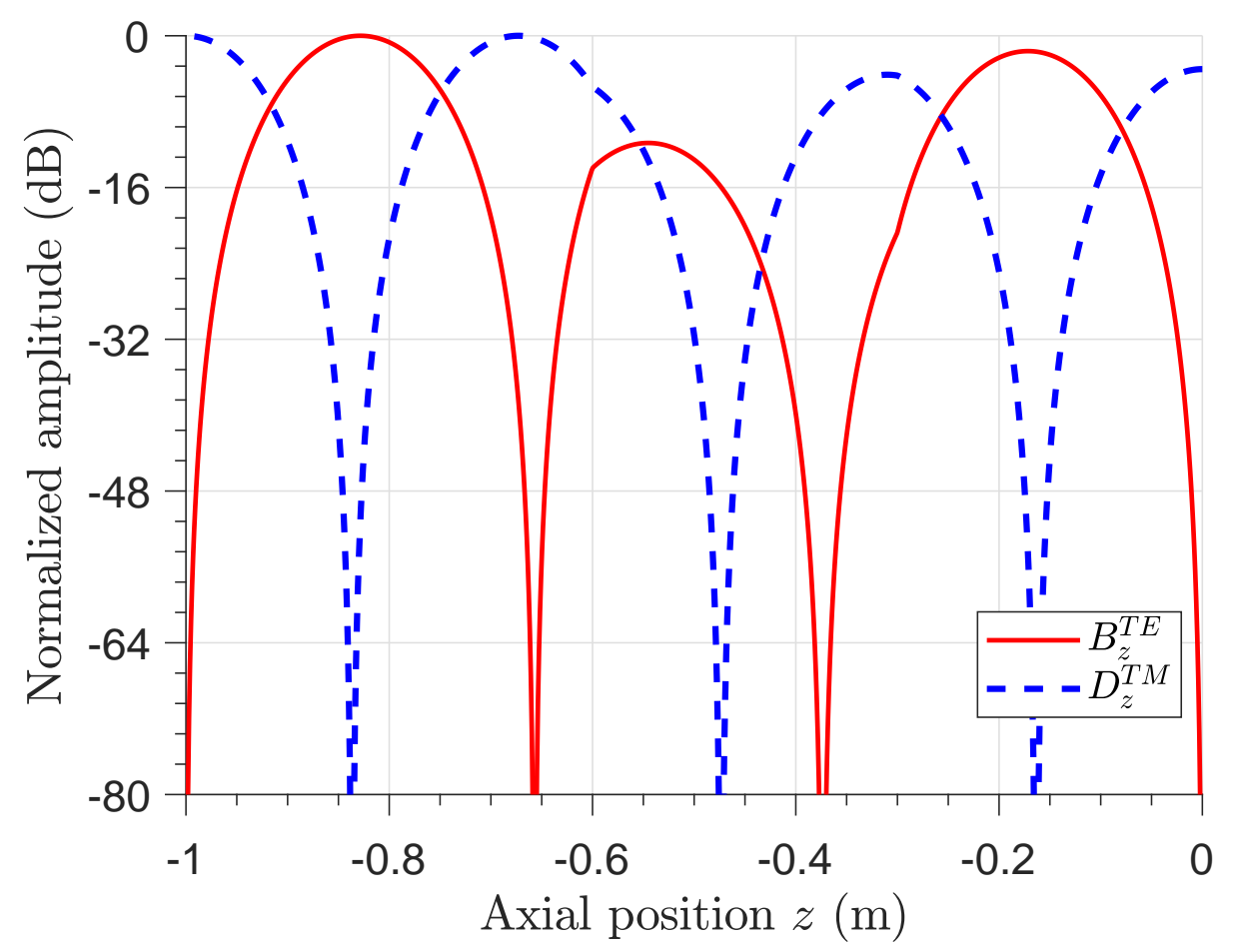

Figure 4.45: Normalized amplitude of the electric flux density and magnetic flux density $\left(D_{z}\right.$ and $\left.B_{z}\right)$ in axially-stratified three-layers isotropic medium (Third Mode).

Table 4.10: Physical characteristics of an axially-stratified five-layers anisotropic medium.

\begin{tabular}{c|cl|cc}
\hline \hline Layer & \multicolumn{2}{|c|}{ Relative Magnetic Permeability } & Relative Electric Permittivity \\
\hline \hline 2 & $\mu_{r s}=1$ & $\mu_{r z}=5$ & $\epsilon_{r s}=6$ & $\epsilon_{r z}=10$ \\
\hline 3 & $\mu_{r s}=2$ & $\mu_{r z}=4$ & $\epsilon_{r s}=7$ & $\epsilon_{r z}=9$ \\
\hline 4 & $\mu_{r s}=3$ & $\mu_{r z}=3.5$ & $\epsilon_{r s}=8$ & $\epsilon_{r z}=8.5$ \\
\hline 5 & $\mu_{r s}=4$ & $\mu_{r z}=2$ & $\epsilon_{r s}=9$ & $\epsilon_{r z}=7$ \\
\hline 6 & $\mu_{r s}=5$ & $\mu_{r z}=1$ & $\epsilon_{r s}=10$ & $\epsilon_{r z}=6$ \\
\hline \hline
\end{tabular}

\subsection{2}

\section{Lossy Media}

In this section we approach the last example analyzed in the previous section for an axially-stratified five-layers isotropic medium, but considering the lossy layer 4 . We explore the first five modes for a conductivity range from $10^{-6}$ to $1 \mathrm{~S} / \mathrm{m}$ After considering lossy formation, the eigenvalues are no longer pure imaginary, but complex. The resulting eigenvalues for these conductivity values have no significant difference for TE mode. This can be appreciated in Fig. 4.55, where as the the conductivity increases, the eigenvalues move 


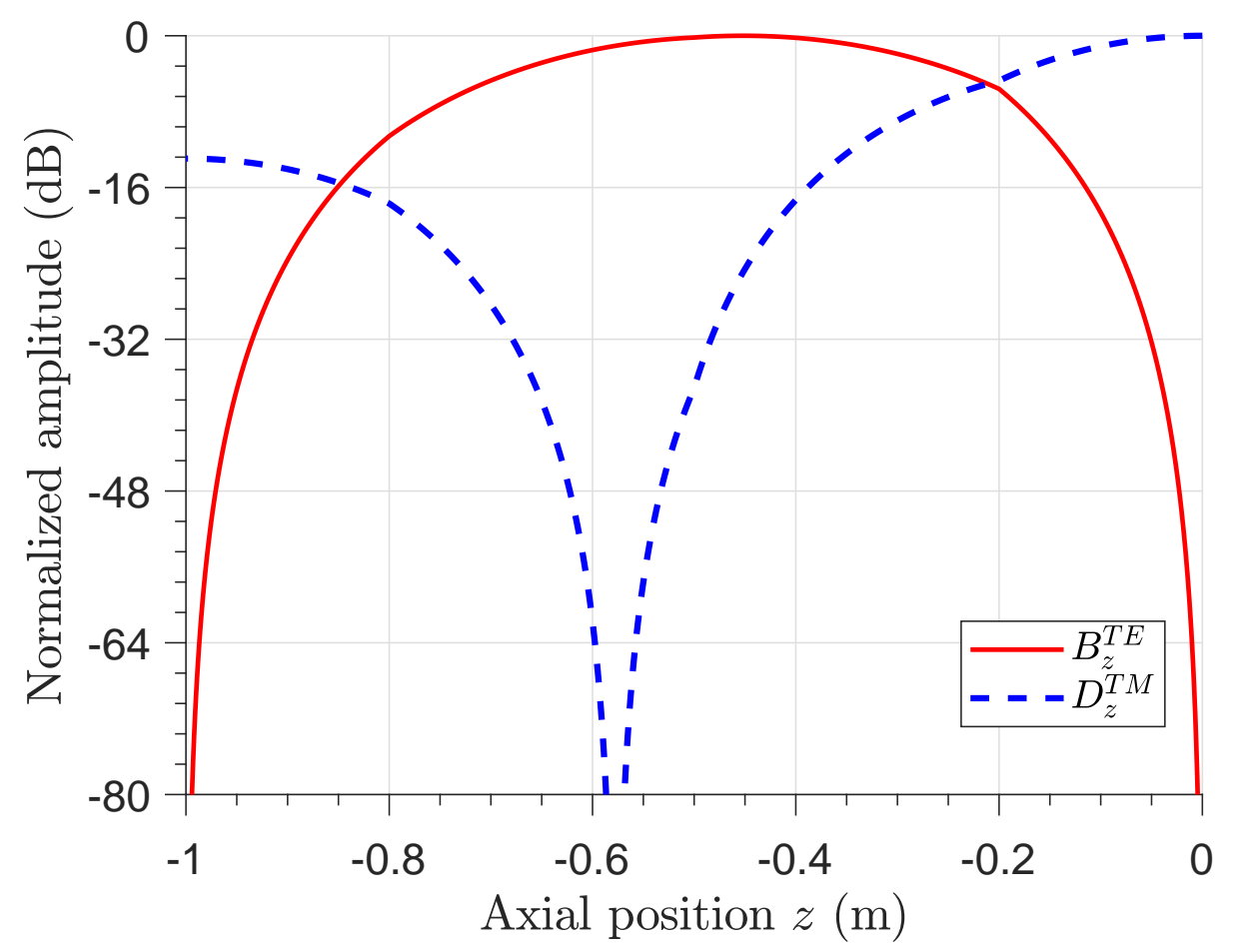

Figure 4.46: Normalized amplitude of the electric flux density and magnetic flux density $\left(D_{z}\right.$ and $\left.B_{z}\right)$ in axially-stratified four-layers isotropic medium (First Mode).

Table 4.11: Eigenvalues for an axially-stratified five-layers anisotropic medium.

\begin{tabular}{c|c|c}
\hline \hline \multirow{2}{*}{ Mode Number } & \multicolumn{2}{|c}{ Eigenvalue $\boldsymbol{k}_{\boldsymbol{\rho}, \boldsymbol{n} \boldsymbol{p}}$} \\
\cline { 2 - 3 } & TE & TM \\
\hline \hline 1st. & $2.855257159257028 \mathrm{i}$ & $3.068312891519532 \mathrm{i}$ \\
\hline 2nd. & $5.564013261795316 \mathrm{i}$ & $6.229140791486254 \mathrm{i}$ \\
\hline 3rd. & $8.354410110114189 \mathrm{i}$ & $9.332272597089041 \mathrm{i}$ \\
\hline 4th. & $10.941861293126705 \mathrm{i}$ & $12.435983855447910 \mathrm{i}$ \\
\hline 5th. & $13.809233407332959 \mathrm{i}$ & $15.551417790948074 \mathrm{i}$ \\
\hline \hline
\end{tabular}

away from the axis of pure imaginary values, but only the real part varies. For demonstrating the non-influence in TE mode, Fig. 4.56 shows how the magnetic flux density, obtained from the second eigenvalue, does not vary, from the lossless case to a conductivity value of $1 \mathrm{~S} / \mathrm{m}$.

On the other hand, the conductivity does have a relevant influence on the TM mode, which is evidenced in the equation (3-8). Some eigenvalues obtained for the TM mode are presented from Table 4.12 to Table 4.18, varying the conductivity value in the range mentioned above. The behavior of these eigenvalues for all conductivity values are shown in Fig. 4.57. With the aim of a more detailed appreciation of this behavior, each eigenvalue is showed from 


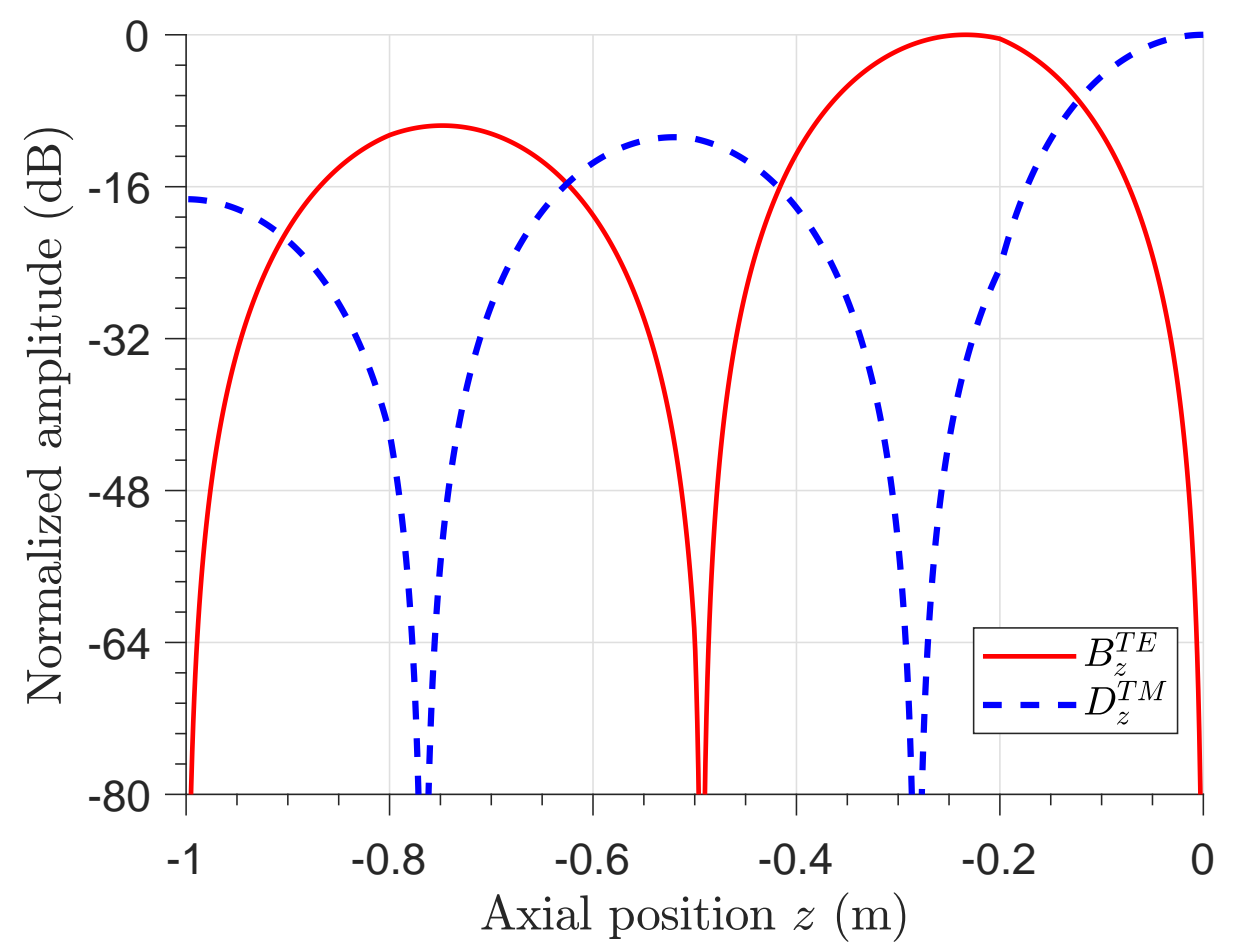

Figure 4.47: Normalized amplitude of the electric flux density and magnetic flux density $\left(D_{z}\right.$ and $\left.B_{z}\right)$ in axially-stratified four-layers isotropic medium (Second Mode).

Table 4.12: Eigenvalues for an axially-stratified five-layers isotropic medium with $\sigma=10^{-6} \mathrm{~S} / \mathrm{m}$ (TM mode).

\begin{tabular}{c|c}
\hline \hline Mode Number & Eigenvalue $\boldsymbol{k}_{\boldsymbol{\rho}, \boldsymbol{n} \boldsymbol{p}}$ \\
\hline \hline 1st. & $0.010737170690891+2.320791056432697 \mathrm{i}$ \\
\hline 2nd. & $-0.015546833948450+6.645255941964114 \mathrm{i}$ \\
\hline 3rd. & $0.015546926989426+9.062689064831508 \mathrm{i}$ \\
\hline 4th. & $-0.010736985198983+13.387154028176727 \mathrm{i}$ \\
\hline 5th. & $0.000000004579974+15.707960413299830 \mathrm{i}$ \\
\hline \hline
\end{tabular}

Fig. 4.58 to Fig. 4.62. For those eigenvalues with the positive real part for each conductivity values (eigenvalue 1, 3 and 5), it is plotted on a logarithmic scale for a best visualization by the reader. In this way, we can easily appreciate that when there are no losses, our eigenvalue is purely imaginary as expected. Finally, the Fig. 4.63 shows the electric flux density for the second eigenvalue, taking into account the lossless case and for conductivity values of $10^{-4}$ and $1 \mathrm{~S} / \mathrm{m}$. We can then perceive the attenuation in field amplitude as conductivity increases. 


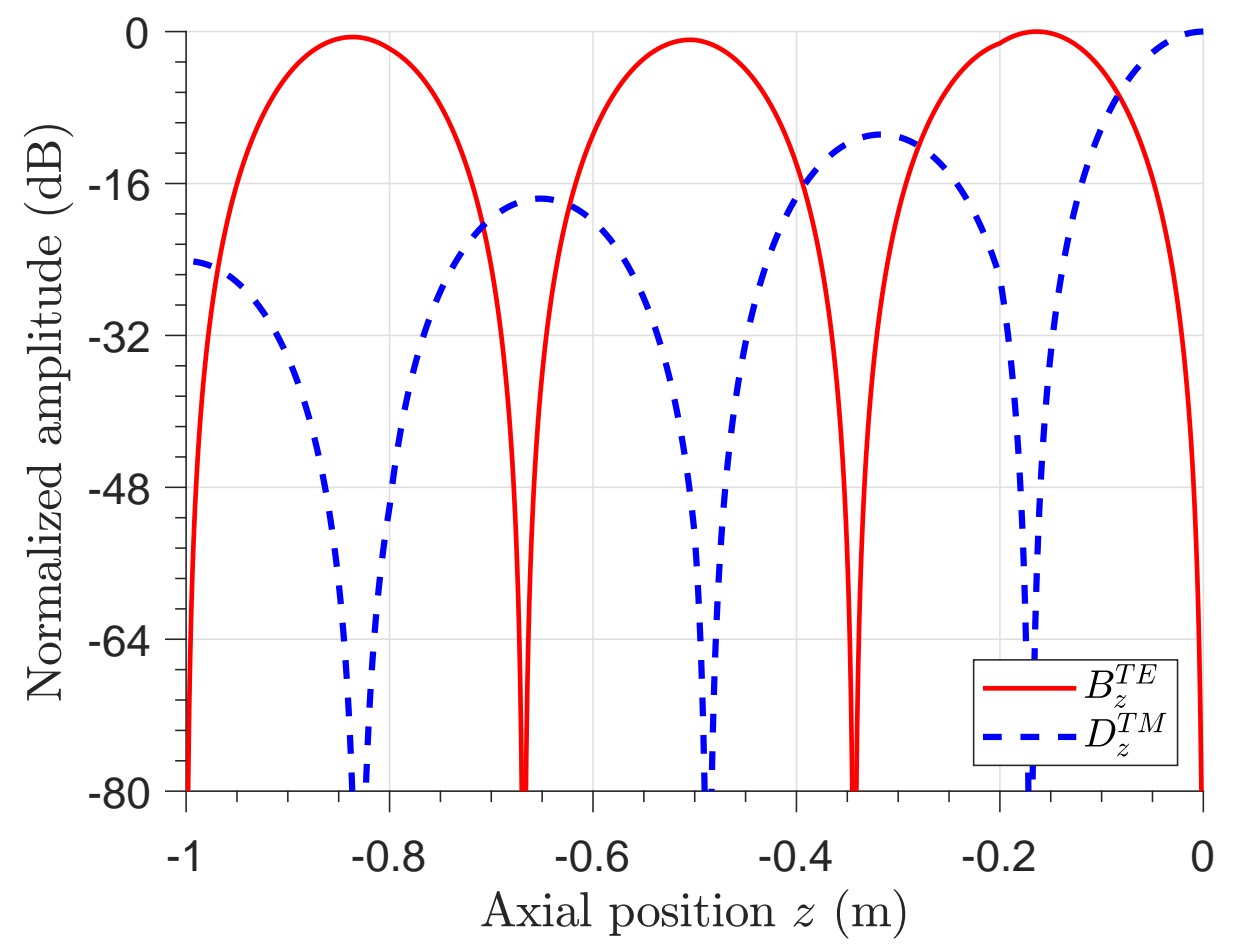

Figure 4.48: Normalized amplitude of the electric flux density and magnetic flux density $\left(D_{z}\right.$ and $\left.B_{z}\right)$ in axially-stratified four-layers isotropic medium (Third Mode).

Table 4.13: Eigenvalues for an axially-stratified five-layers isotropic medium with $\sigma=10^{-5} \mathrm{~S} / \mathrm{m}$ (TM mode).

\begin{tabular}{c|c}
\hline \hline Mode Number & Eigenvalue $\boldsymbol{k}_{\boldsymbol{\rho}, \boldsymbol{n} \boldsymbol{p}}$ \\
\hline \hline 1st. & $0.106862328392550+2.313691317126018 \mathrm{i}$ \\
\hline 2nd. & $-0.153006065911827+6.664596046384765 \mathrm{i}$ \\
\hline 3rd. & $0.153006983879134+9.043348851454198 \mathrm{i}$ \\
\hline 4th. & $-0.106860469982018+13.394253777757495 \mathrm{i}$ \\
\hline 5th. & $0.000000044664362+15.707960406215266 \mathrm{i}$ \\
\hline \hline
\end{tabular}

Table 4.14: Eigenvalues for an axially-stratified five-layers isotropic medium with $\sigma=10^{-4} \mathrm{~S} / \mathrm{m}$ (TM mode).

\begin{tabular}{c|c}
\hline \hline Mode Number & Eigenvalue $\boldsymbol{k}_{\rho, n p}$ \\
\hline \hline 1st. & $0.735462716208924+1.847739342205968 \mathrm{i}$ \\
\hline 2nd. & $-0.588756636944571+7.402333434332047 \mathrm{i}$ \\
\hline 3rd. & $0.588760309931038+8.305606893664212 \mathrm{i}$ \\
\hline 4th. & $-0.735443155911522+13.860204830215682 \mathrm{i}$ \\
\hline 5th. & $0.000000128382604+15.707960207626666 \mathrm{i}$ \\
\hline \hline
\end{tabular}




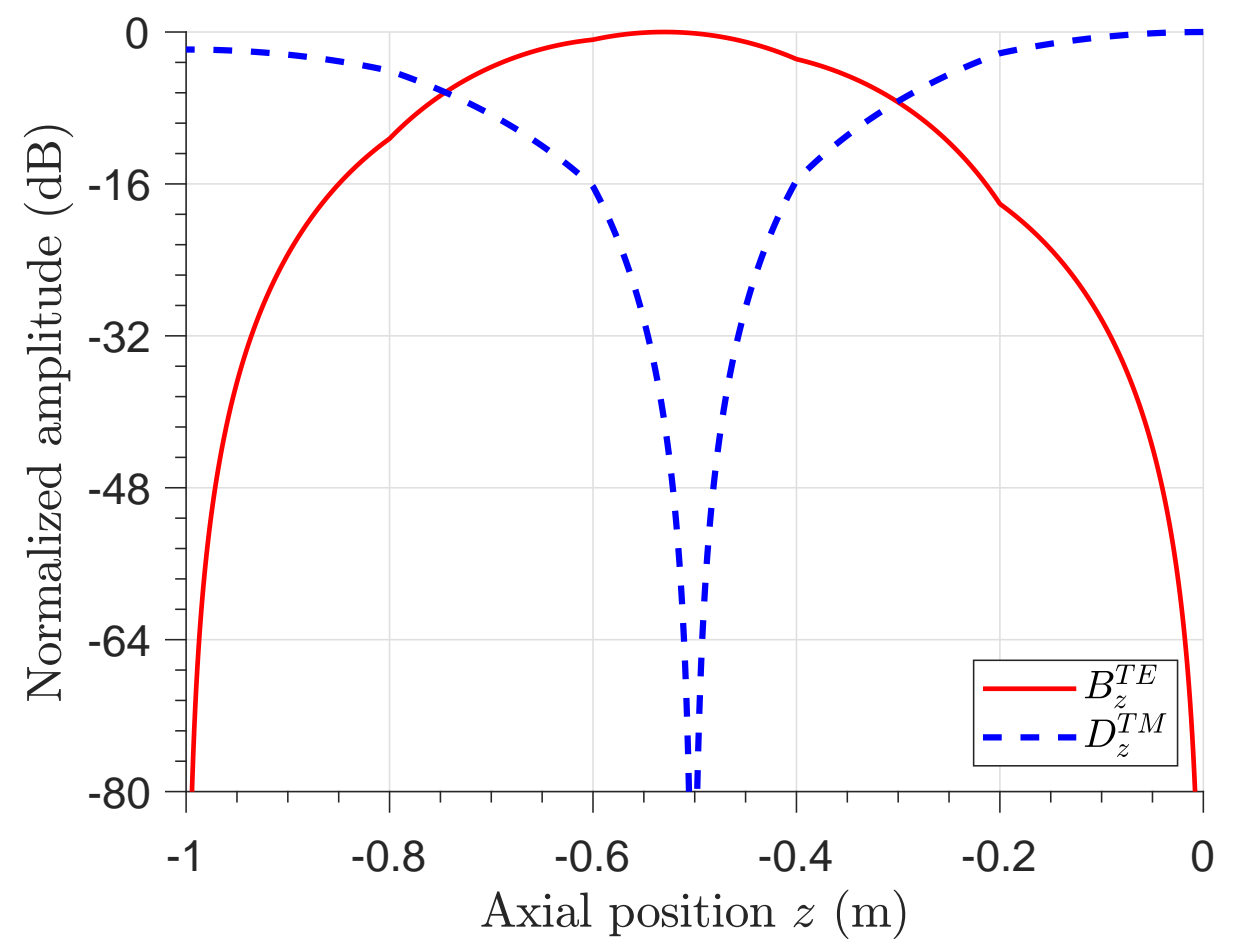

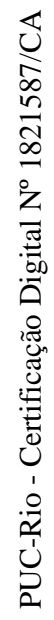

Figure 4.49: Normalized amplitude of the electric flux density and magnetic flux density $\left(D_{z}\right.$ and $\left.B_{z}\right)$ in axially-stratified five-layers isotropic medium (First Mode).

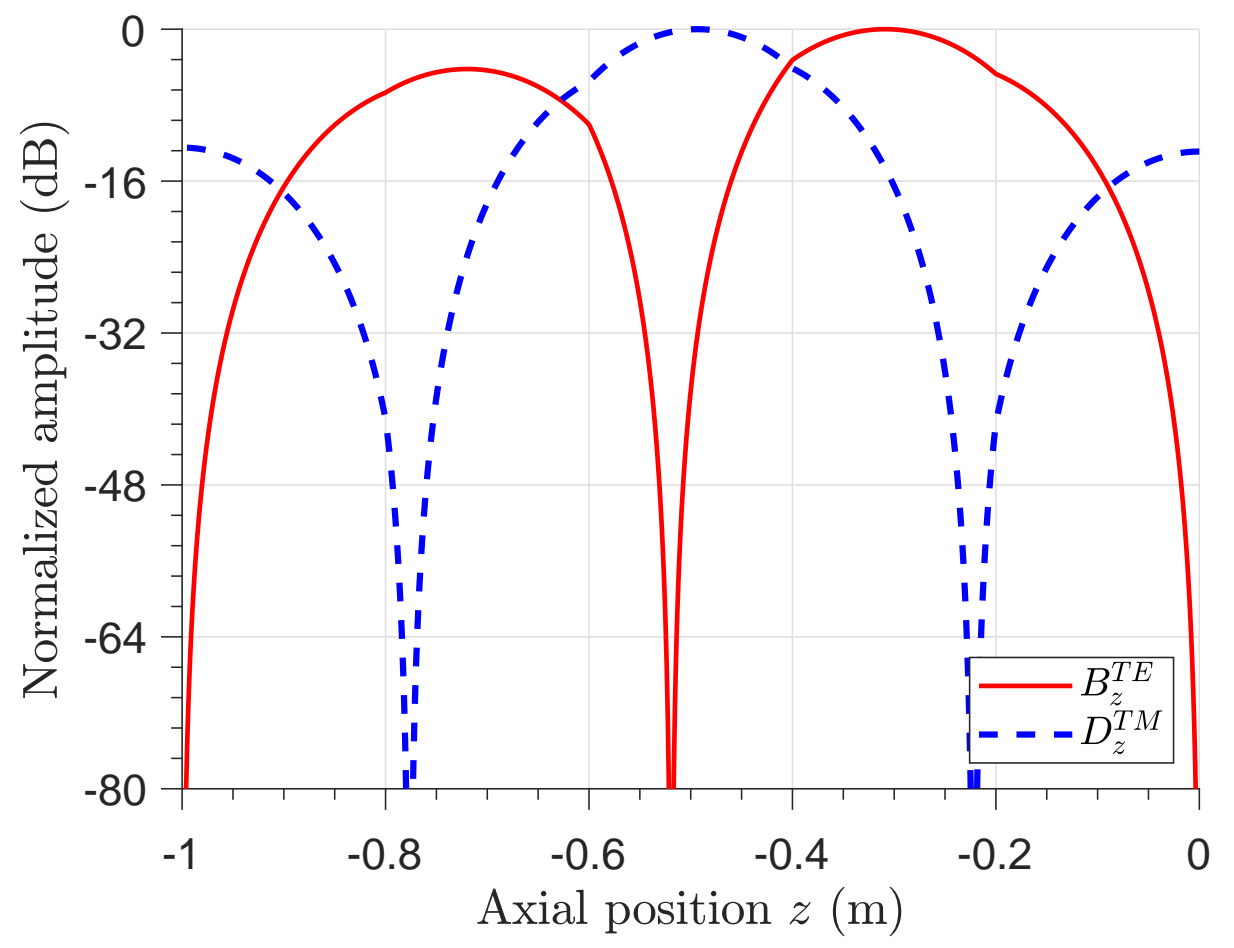

Figure 4.50: Normalized amplitude of the electric flux density and magnetic flux density $\left(D_{z}\right.$ and $\left.B_{z}\right)$ in axially-stratified five-layers isotropic medium (Second Mode). 


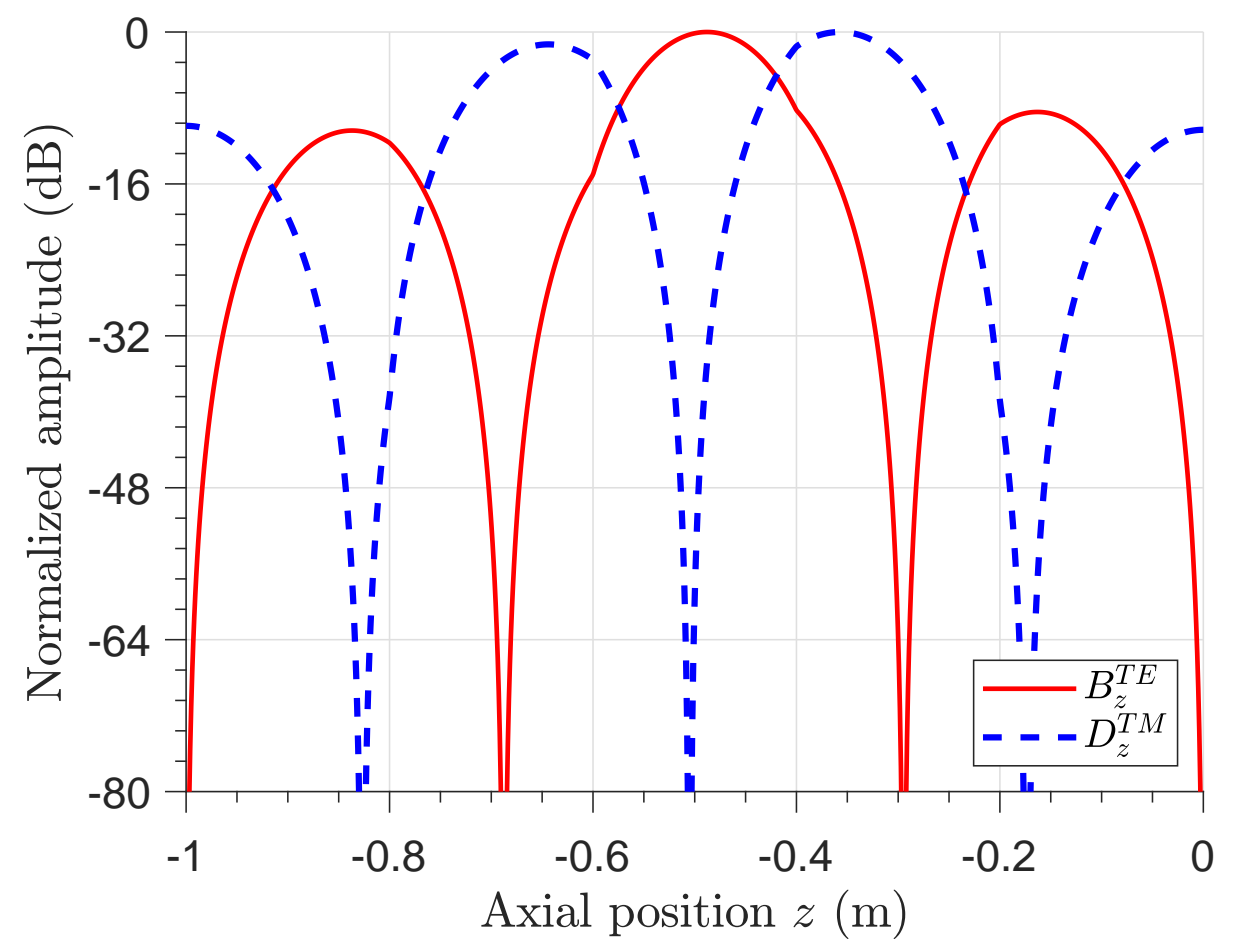

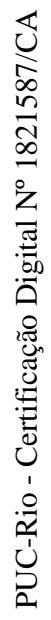

Figure 4.51: Normalized amplitude of the electric flux density and magnetic flux density $\left(D_{z}\right.$ and $\left.B_{z}\right)$ in axially-stratified five-layers isotropic medium (Third Mode).

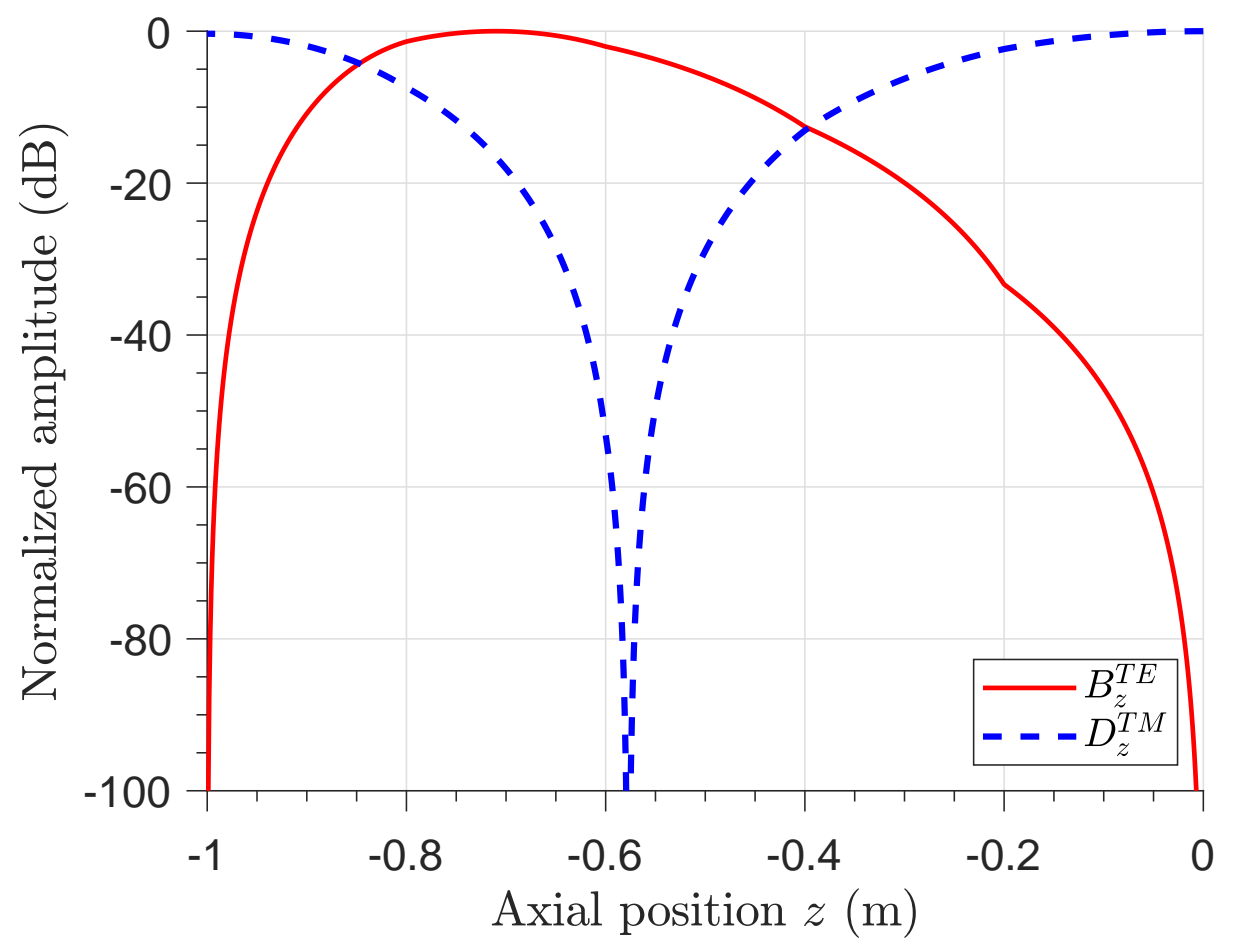

Figure 4.52: Normalized amplitude of the electric flux density and magnetic flux density $\left(D_{z}\right.$ and $\left.B_{z}\right)$ in axially-stratified five-layers anisotropic medium (First Mode). 


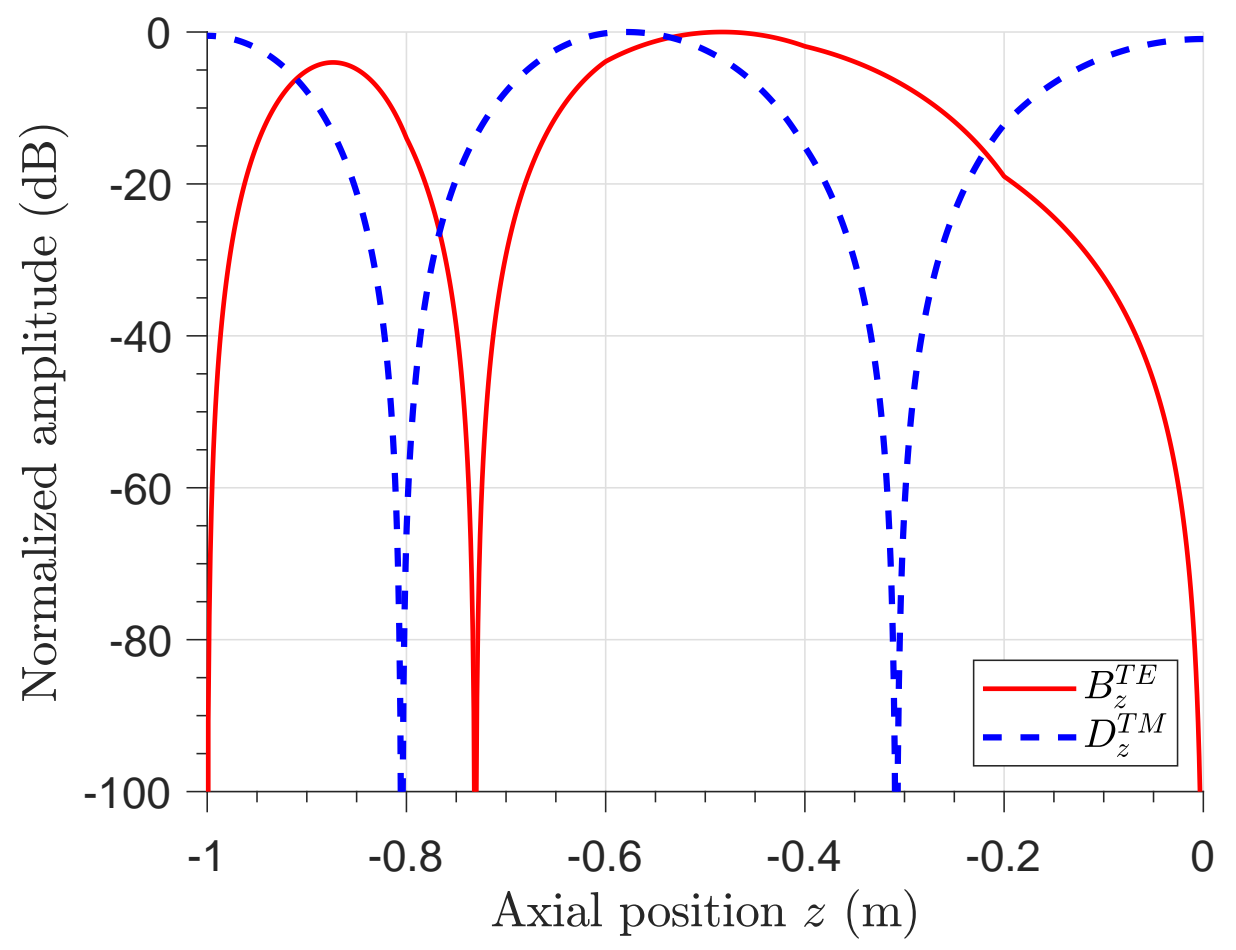

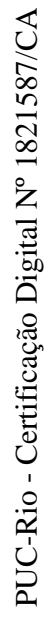

Figure 4.53: Normalized amplitude of the electric flux density and magnetic flux density $\left(D_{z}\right.$ and $\left.B_{z}\right)$ in axially-stratified five-layers anisotropic medium (Second Mode).

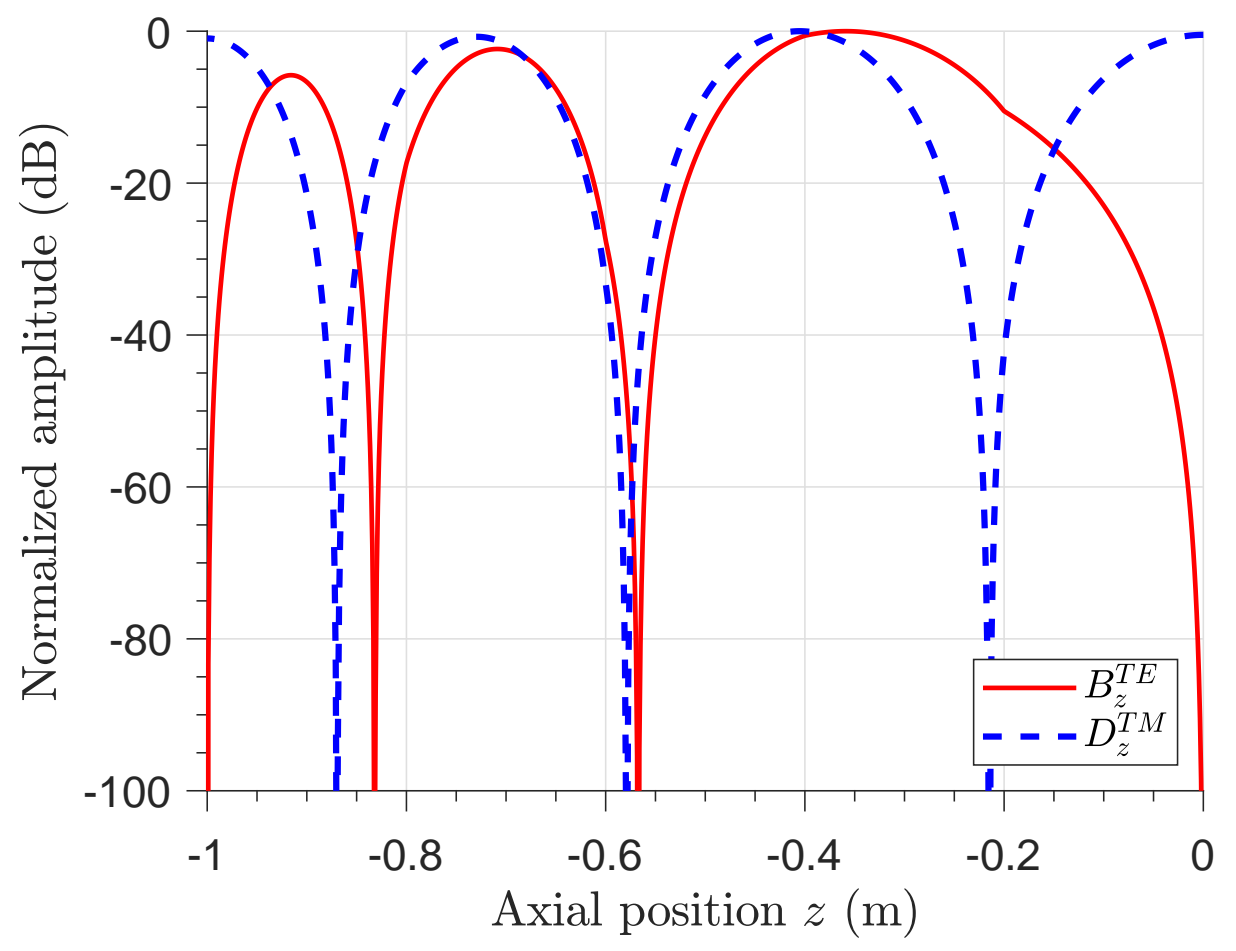

Figure 4.54: Normalized amplitude of the electric flux density and magnetic flux density $\left(D_{z}\right.$ and $\left.B_{z}\right)$ in axially-stratified five-layers anisotropic medium (Third Mode). 


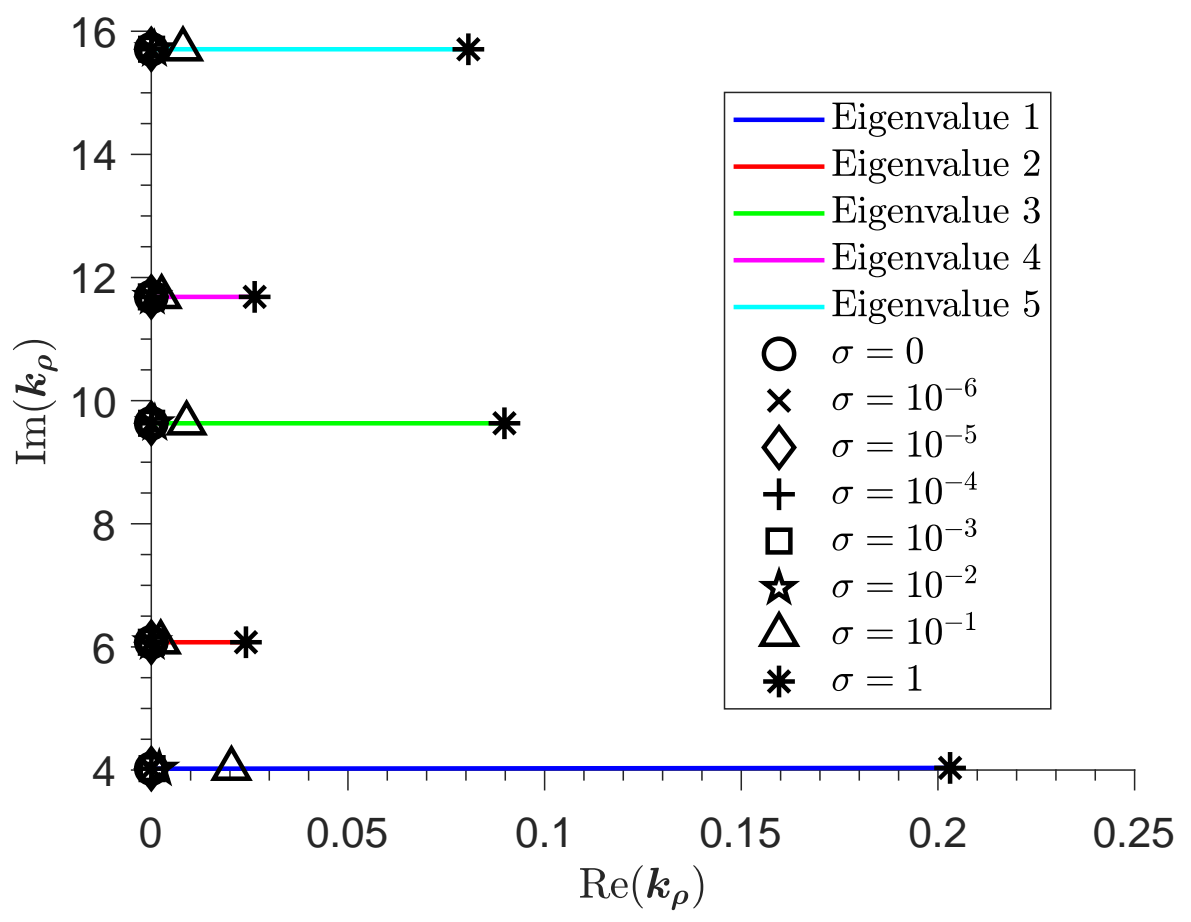

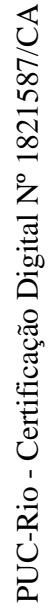

Figure 4.55: Eigenvalue behavior to several conductivity values for TE mode.

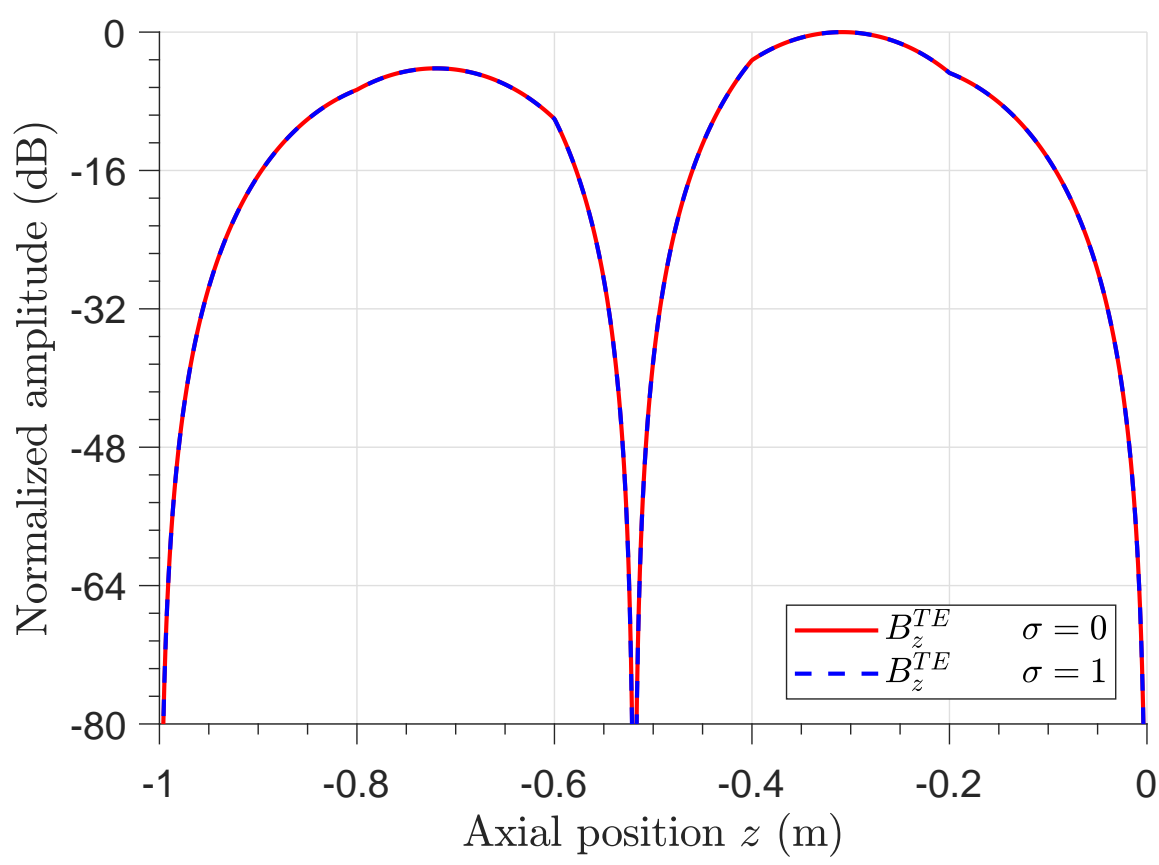

Figure 4.56: Non-Variation of the magnetic flux density $\left(B_{z}\right)$ to different two conductivity values in axially-stratified five-layers isotropic medium (Second Mode). 
Table 4.15: Eigenvalues for an axially-stratified five-layers isotropic medium with $\sigma=10^{-3} \mathrm{~S} / \mathrm{m}$ (TM mode).

\begin{tabular}{c|c}
\hline \hline Mode Number & Eigenvalue $\boldsymbol{k}_{\boldsymbol{\rho}, \boldsymbol{n} \boldsymbol{p}}$ \\
\hline \hline 1st. & $0.504012679965596+0.558822120667639 \mathrm{i}$ \\
\hline 2nd. & $-0.092319353860095+7.847004086074592 \mathrm{i}$ \\
\hline 3rd. & $0.092319889254419+7.860933613498648 \mathrm{i}$ \\
\hline 4th. & $-0.503866005425398+15.149095730954890 \mathrm{i}$ \\
\hline 5th. & $0.000000017775623+15.707960128627615 \mathrm{i}$ \\
\hline
\end{tabular}

Table 4.16: Eigenvalues for an axially-stratified five-layers isotropic medium with $\sigma=10^{-2} \mathrm{~S} / \mathrm{m}$ (TM mode).

\begin{tabular}{c|c}
\hline \hline Mode Number & Eigenvalue $\boldsymbol{k}_{\boldsymbol{\rho}, \boldsymbol{n} \boldsymbol{p}}$ \\
\hline \hline 1st. & $0.167934549018248+0.169461496532328 \mathrm{i}$ \\
\hline 2nd. & $-0.009282484592447+7.853893887725020 \mathrm{i}$ \\
\hline 3rd. & $0.009282542492692+7.854043777543839 \mathrm{i}$ \\
\hline 4th. & $-0.166748525855869+15.538368003891952 \mathrm{i}$ \\
\hline 5th. & $0.000000001864182+15.707960127530091 \mathrm{i}$ \\
\hline \hline
\end{tabular}

Table 4.17: Eigenvalues for an axially-stratified five-layers isotropic medium with $\sigma=10^{-1} \mathrm{~S} / \mathrm{m}$ (TM mode).

\begin{tabular}{c|c}
\hline \hline Mode Number & Eigenvalue $\boldsymbol{k}_{\boldsymbol{\rho}, \boldsymbol{n} \boldsymbol{p}}$ \\
\hline \hline 1st. & $0.053669001261055+0.053031374899822 \mathrm{i}$ \\
\hline 2nd. & $-0.000928257971985+7.853963142463697 \mathrm{i}$ \\
\hline 3rd. & $0.000928305655962+7.853974522607146 \mathrm{i}$ \\
\hline 4th. & $-0.043218390059025+15.655038710909594 \mathrm{i}$ \\
\hline 5th. & $0.000000000984145+15.707960127519843 \mathrm{i}$ \\
\hline \hline
\end{tabular}

Table 4.18: Eigenvalues for an axially-stratified five-layers isotropic medium with $\sigma=1 \mathrm{~S} / \mathrm{m}$ (TM mode).

\begin{tabular}{c|c}
\hline \hline Mode Number & Eigenvalue $\boldsymbol{k}_{\boldsymbol{\rho}, \boldsymbol{n} \boldsymbol{p}}$ \\
\hline \hline 1st. & $0.017998432729488+0.015813829345154 \mathrm{i}$ \\
\hline 2nd. & $-0.000092413590718+7.853963849232947 \mathrm{i}$ \\
\hline 3rd. & $0.000092836780005+7.853973830341202 \mathrm{i}$ \\
\hline 4th. & $0.000020208404332+15.705264225732185 \mathrm{i}$ \\
\hline 5th. & $0.000000008075114+15.707960127596873 \mathrm{i}$ \\
\hline \hline
\end{tabular}




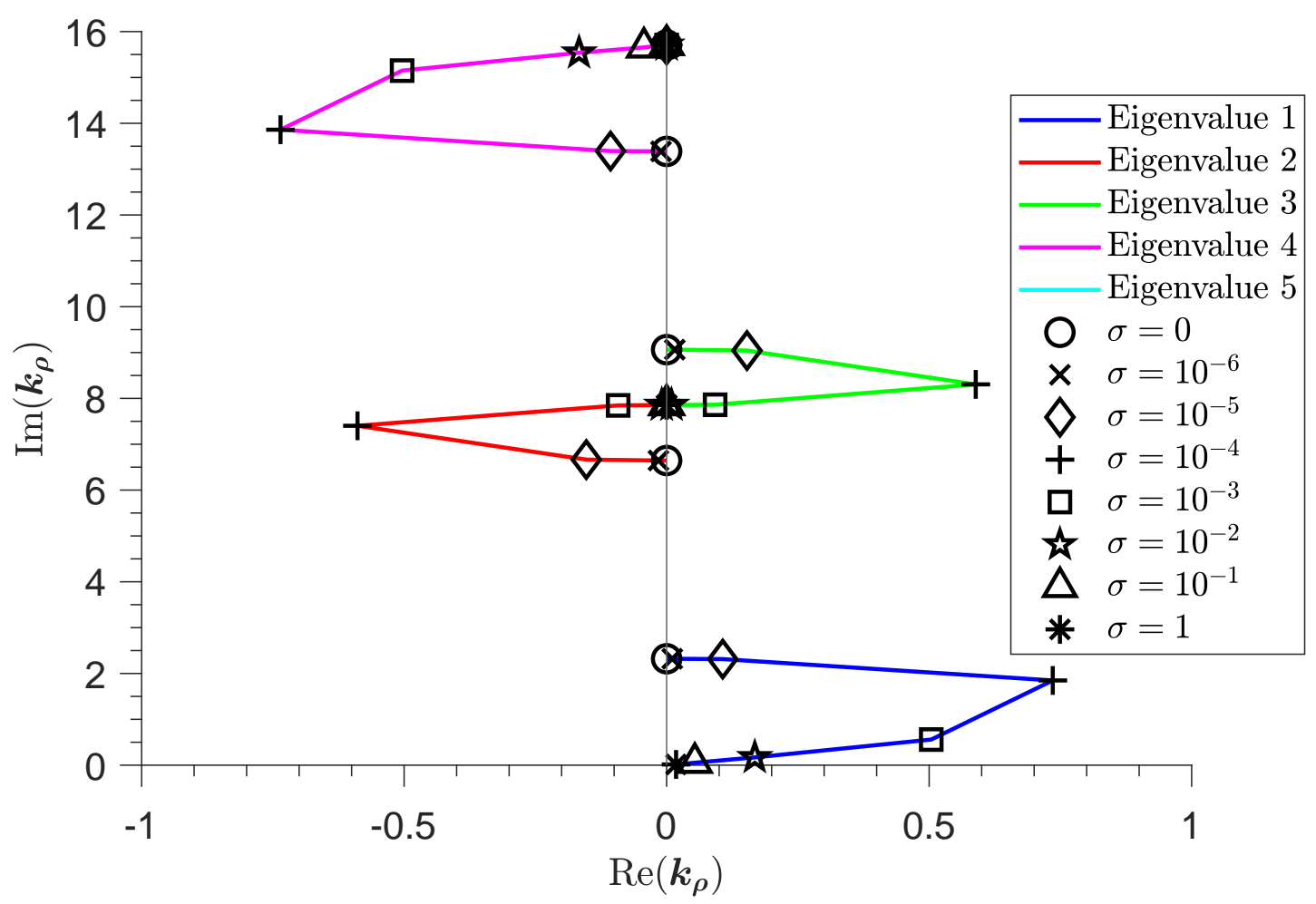

Figure 4.57: Eigenvalue behavior to several conductivity values for TM mode.

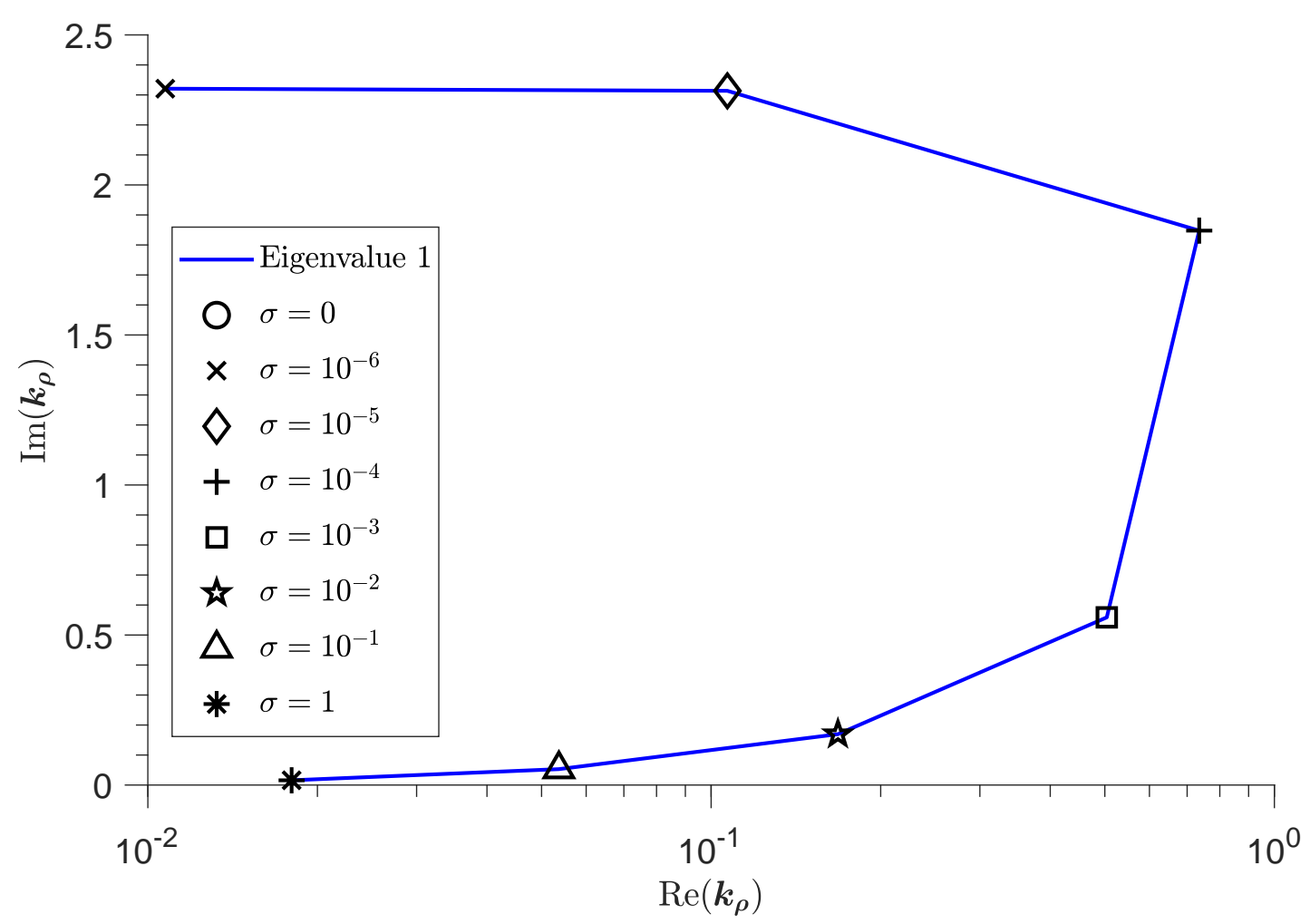

Figure 4.58: Behavior of the first eigenvalue to several conductivity values for TM mode. (Zoom of the eigenvalue 1 from Fig. 4.57). 


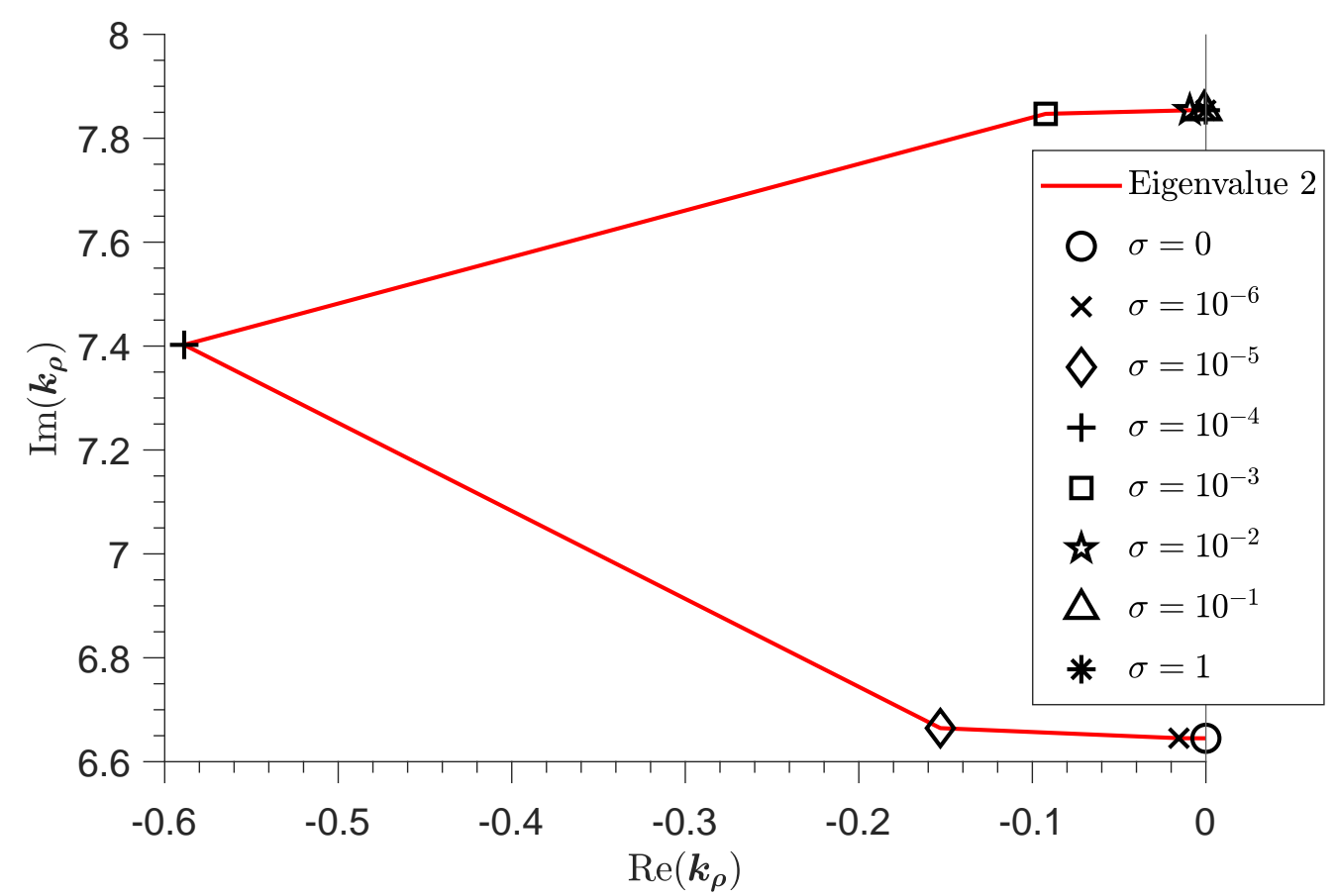

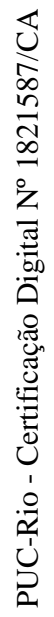

Figure 4.59: Behavior of the second eigenvalue to several conductivity values for TM mode. (Zoom of the eigenvalue 2 from Fig. 4.57).

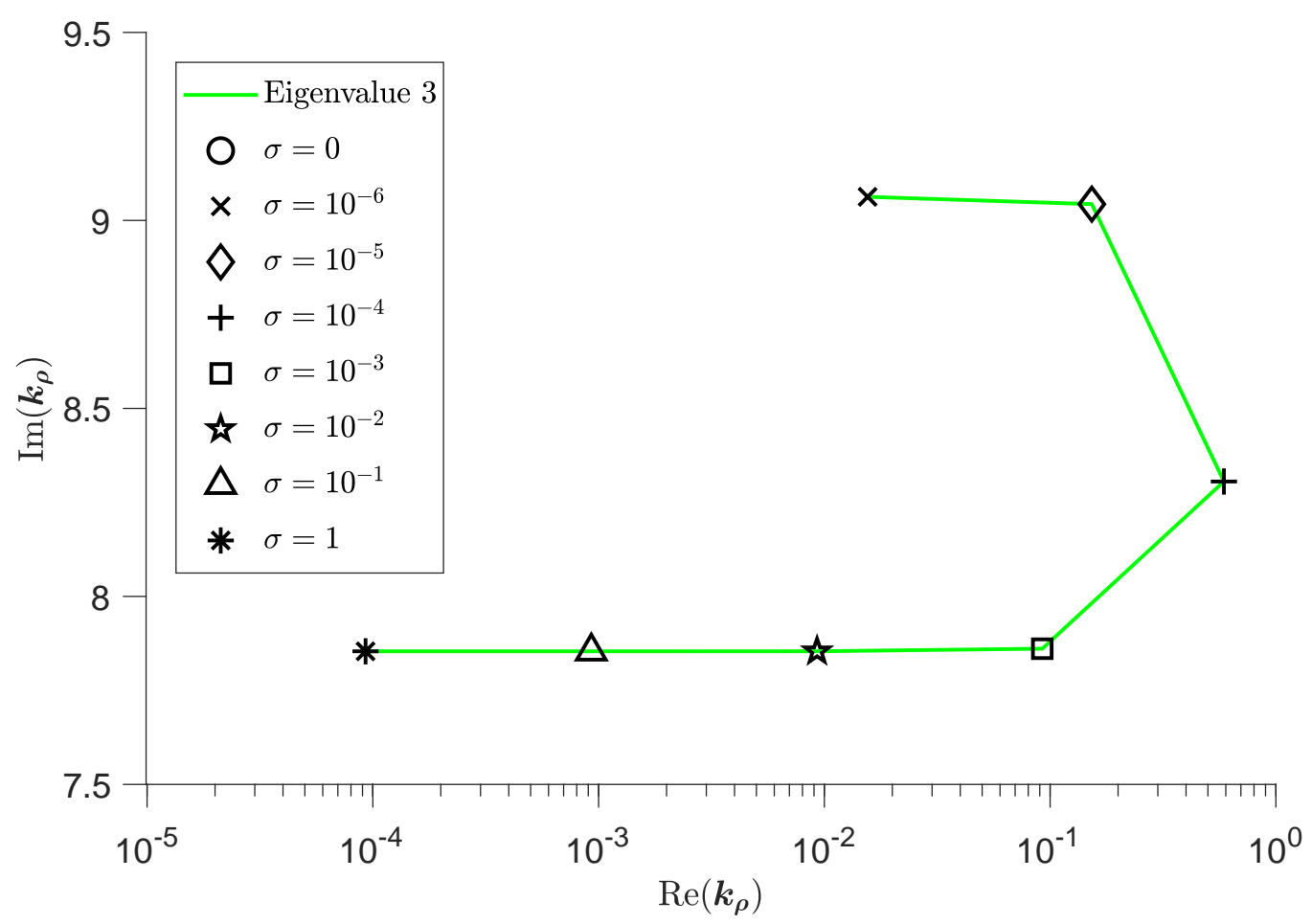

Figure 4.60: Behavior of the third eigenvalue to several conductivity values for TM mode. (Zoom of the eigenvalue 3 from Fig. 4.57). 


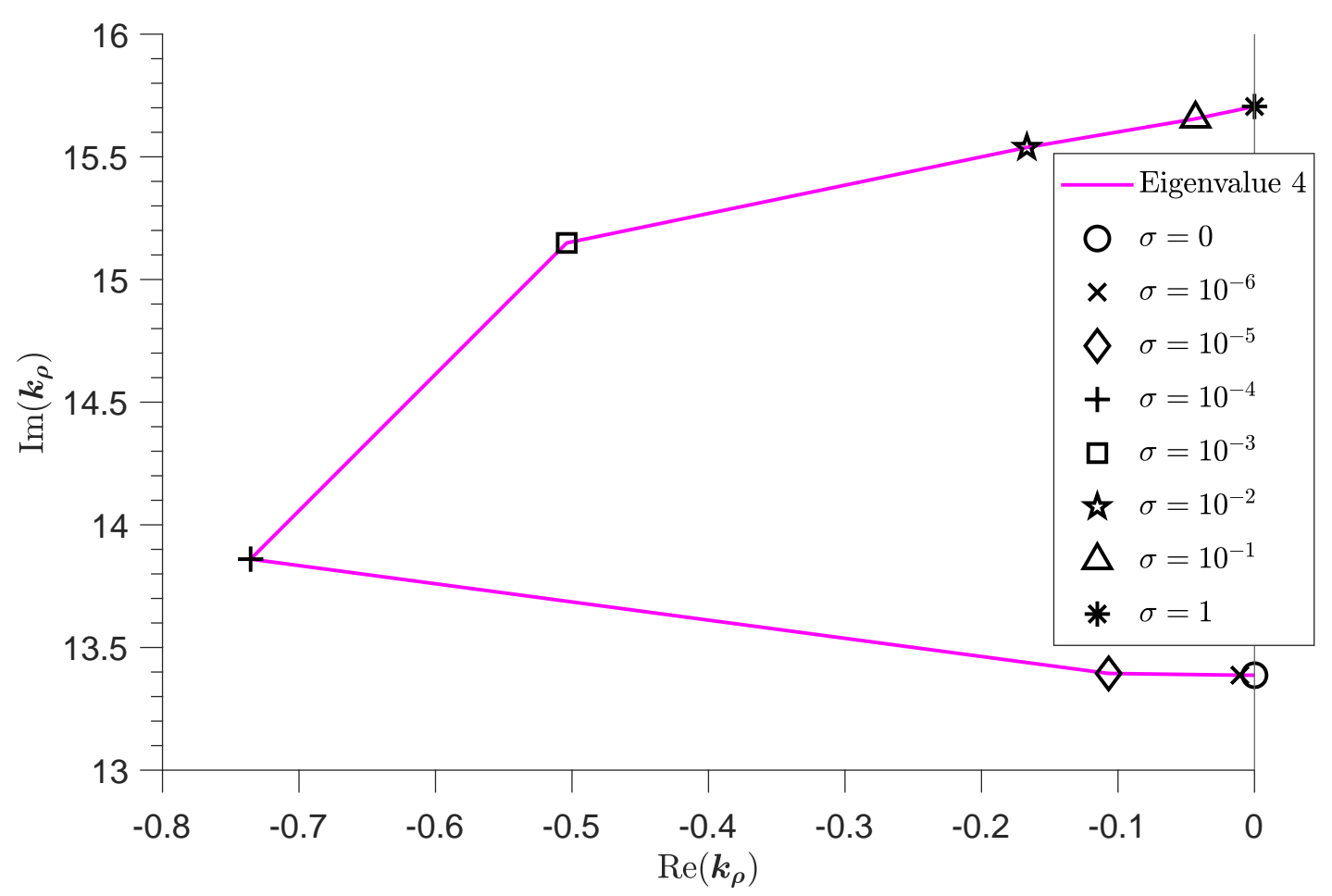

Figure 4.61: Behavior of the fourth eigenvalue to several conductivity values for TM mode. (Zoom of the eigenvalue 4 from Fig. 4.57).

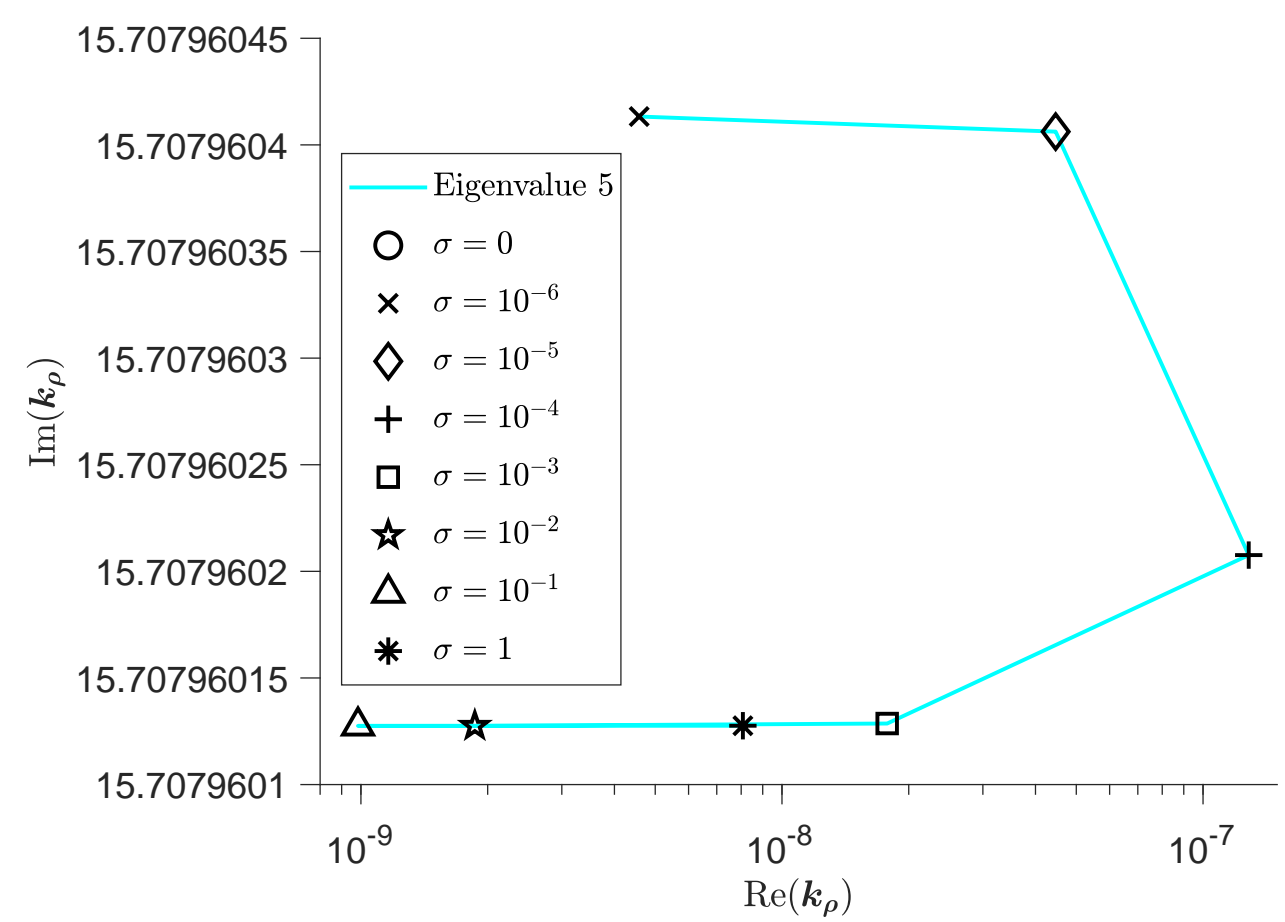

Figure 4.62: Behavior of the fifth eigenvalue to several conductivity values for TM mode. (Zoom of the eigenvalue 5 from Fig. 4.57). 


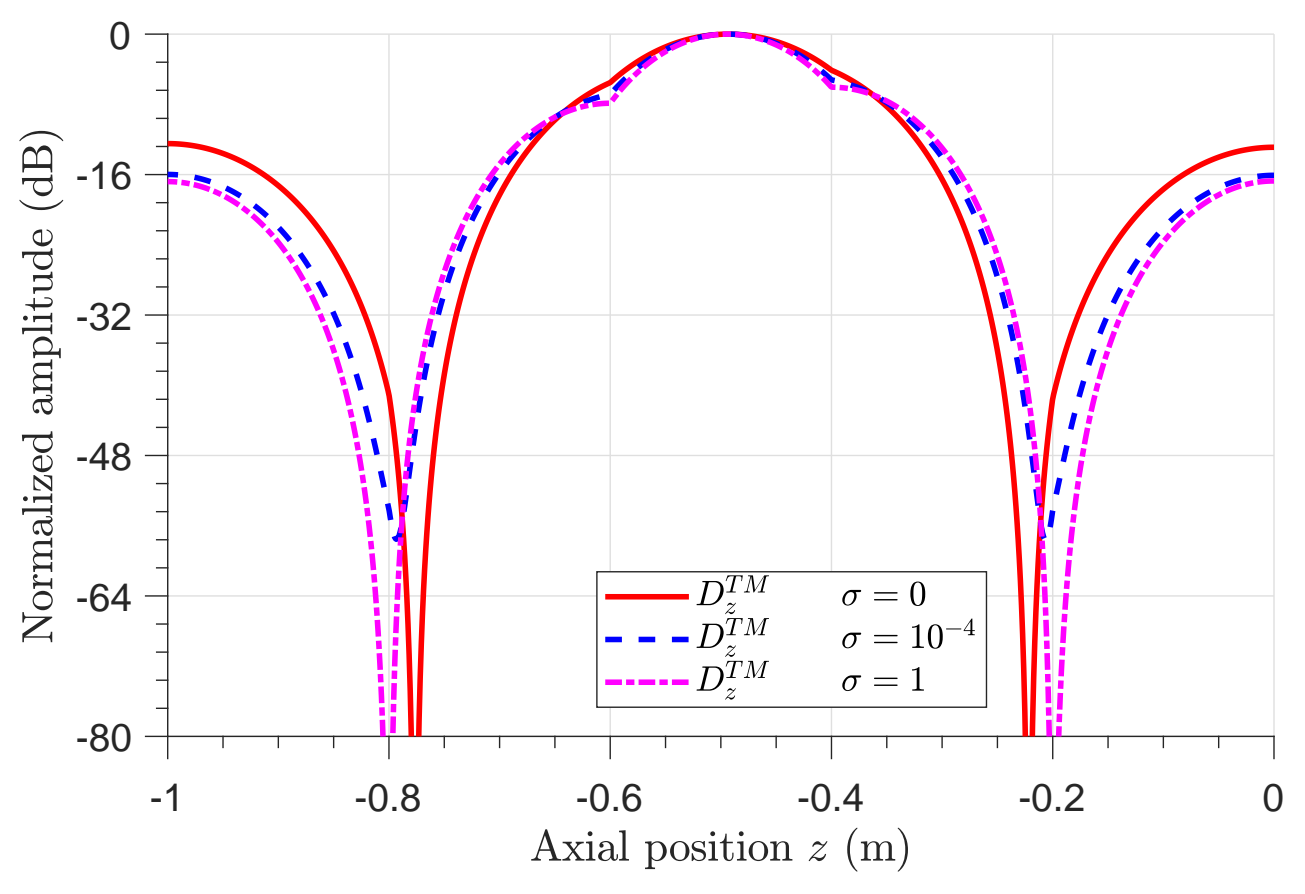

Figure 4.63: Variation of the electric flux density $\left(D_{z}\right)$ to several conductivity values in axially-stratified five-layers isotropic medium (Second Mode). 


\section{Source Expansion}

\section{1}

\section{Introduction}

Conventional well-logging tools employing horizontal-coil antenna excite only TE modes (i.e. perpendicular to the tool axis) as is illustrated in Fig. 5.1. Logging tools with tilted-coil antenna have been proposed as an alternative to

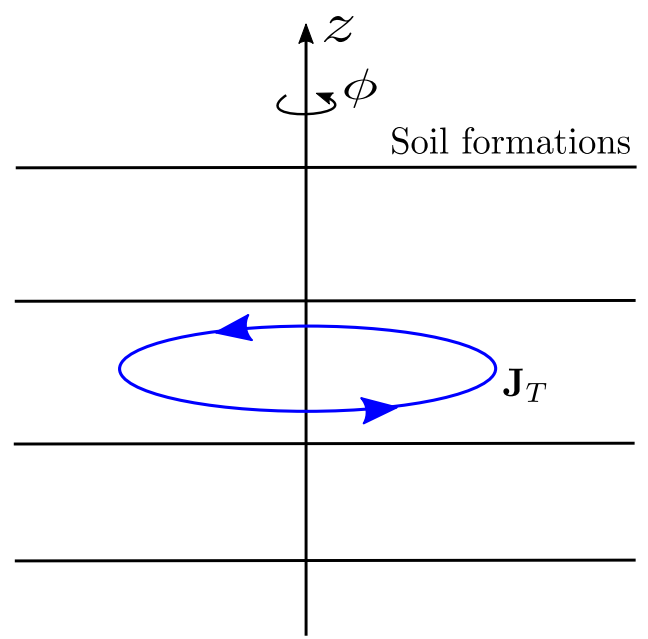

Figure 5.1: Electric current horizontal source within an axially-stratified geophysical formation.

provide better evaluation in multilayered complex anisotropic formations [56]. In this chapter, we will study the response of induction due to a finite coil model in homogeneous formations. We will present the derivation of formulas for to find the amplitudes delivered by a uniform azimuthally symmetric electric current in a tilted loop source that is embedded in an arbitrary layer of a multilayered-uniaxial-anisotropic formation. We assume the antenna is always completely within the same layer. The solution will be obtained from Maxwell's equations and Sturm-Liouville theory. In order to apply this theory, it will be necessary to formulate the generalized Fourier series, analogous to the transform presented in [11]. Finally, we will apply the Sturm-Liouville theory, where a similar procedure is aboard in [49] but for an isotropic medium. 


\section{2}

\section{Modal Field Excitation}

The Maxwell's equations in linear, homogeneous and dissipative anisotropic media are given by

$$
\begin{aligned}
\nabla \times \mathbf{E} & =i \omega \overline{\bar{\mu}} \cdot \mathbf{H}-\mathbf{M}_{T}, \\
\nabla \times \mathbf{H} & =-i \omega \overline{\bar{\epsilon}} \cdot \mathbf{E}+\mathbf{J}_{T}, \\
\nabla \cdot(\overline{\bar{\epsilon}} \cdot \mathbf{E}) & =\varrho_{e}, \\
\nabla \cdot(\overline{\bar{\mu}} \cdot \mathbf{H}) & =\varrho_{m} .
\end{aligned}
$$

Taking the curl of the equation (5-2) and projecting the resulting vector onto $\hat{z}$, we can find

$$
\begin{aligned}
\hat{z} \cdot[\boldsymbol{\nabla} \times(\boldsymbol{\nabla} \times \mathbf{H})]= & -i \omega \hat{z} \cdot[\boldsymbol{\nabla} \times(\overline{\bar{\epsilon}} \cdot \mathbf{E})]+\hat{z} \cdot\left(\boldsymbol{\nabla} \times \mathbf{J}_{T}\right) \\
= & -i \omega \hat{z} \cdot\left[\left(\boldsymbol{\nabla}_{s}+\hat{z} \frac{\partial}{\partial z}\right) \times\left(\overline{\bar{\epsilon}}_{s} \mathbf{E}_{s}+\hat{z} \epsilon_{z} E_{z}\right)\right]+\hat{z} \cdot\left\{\left(\boldsymbol{\nabla}_{s} \times \mathbf{J}_{s}\right)+\right. \\
& {\left.\left[\boldsymbol{\nabla}_{s} \times\left(\hat{z} J_{z}\right)\right]+\left(\frac{\partial}{\partial z} \hat{z} \times \mathbf{J}_{s}\right)+\left[\frac{\partial}{\partial z} \hat{z} \times\left(\hat{z} J_{z}\right)\right]\right\} } \\
= & -i \omega \hat{z} \cdot\left[\boldsymbol{\nabla}_{s} \times\left(\overline{\bar{\epsilon}}_{s} \mathbf{E}_{s}\right)\right]+\hat{z}\left(\boldsymbol{\nabla}_{s} \times \mathbf{J}_{s}\right) \\
= & \hat{z} \cdot\left[-i \omega \epsilon_{s}\left(\boldsymbol{\nabla}_{s} \times \mathbf{E}_{s}\right)+\left(\boldsymbol{\nabla}_{s} \times \mathbf{J}_{s}\right)\right]
\end{aligned}
$$

Separating the equation (5-1) in components $\hat{s}$ and $\hat{z}$, we have that

$$
\left\{\begin{array}{ccc}
\hat{s}: & \nabla_{s} \times \hat{z} E_{z}+\frac{\partial}{\partial z} \hat{z} \times \mathbf{E}_{s} & =i \omega \overline{\bar{\mu}} \cdot \mathbf{H}_{s}-\mathbf{M}_{s} \\
\hat{z}: & \nabla_{s} \times \mathbf{E}_{s} & =i \omega \hat{z} \mu_{z} H_{z}-\hat{z} M_{z},
\end{array}\right.
$$

projecting the $\hat{z}$ component of equation (5-6) onto $\hat{z}$ and substituting the result obtained in equation (5-5),

$$
\hat{z} \cdot[\boldsymbol{\nabla} \times(\boldsymbol{\nabla} \times \mathbf{H})]=\omega^{2} \epsilon_{s} \mu_{z} H_{z}+i \omega \epsilon_{s} M_{z}+\hat{z} \cdot\left(\boldsymbol{\nabla}_{s} \times \mathbf{J}_{s}\right) .
$$

After using the vector identity (3-17), the LHS of (5-7) can be simplified to,

$$
\nabla^{2} H_{z}+k^{2} H_{z}=\left[\boldsymbol{\nabla}(\boldsymbol{\nabla} \cdot \mathbf{H})-\left(\boldsymbol{\nabla}_{s} \times \mathbf{J}_{s}\right)\right]_{z}-i \omega \epsilon_{s} M_{z}
$$

Making a same previous procedure on equation (5-1), or by duality with equation (5-8), we can obtain

$$
\nabla^{2} E_{z}+k^{2} E_{z}=\left[\boldsymbol{\nabla}(\boldsymbol{\nabla} \cdot \mathbf{E})+\left(\boldsymbol{\nabla}_{s} \times \mathbf{M}_{s}\right)\right]_{z}-i \omega \mu_{s} J_{z}
$$

Substituting the equations (3-15) and (3-16) into (5-8) and (5-9) respectively, and substituting the scalar Laplacian in cylindrical coordinates given 
by

we have

$$
\nabla^{2}=\nabla_{s}^{2}+\frac{\partial^{2}}{\partial z^{2}}
$$

$$
\begin{gathered}
\nabla_{s}^{2} H_{z}+\frac{\mu_{z}}{\mu_{s}} \frac{\partial^{2} H_{z}}{\partial z^{2}}+k^{2} H_{z}=-\hat{z} \cdot\left(\nabla_{s} \times \mathbf{J}_{s}\right)-i \omega \epsilon_{s} M_{z} \\
\nabla_{s}^{2} E_{z}+\frac{\epsilon_{z}}{\epsilon_{s}} \frac{\partial^{2} E_{z}}{\partial z^{2}}+k^{2} E_{z}=\hat{z} \cdot\left(\nabla_{s} \times \mathbf{M}_{s}\right)-i \omega \mu_{s} J_{z}
\end{gathered}
$$

where $k^{2}=\omega^{2} p_{z} \tilde{p}_{s}$, with $p=\{\epsilon, \mu\}$ and $\tilde{p}=\{\mu, \epsilon\}$.

\section{3}

\section{Current Density}

Considering the tilted-coil transmitting antenna in Fig. 5.2, we can derive its volumetric electric current density $\mathbf{J}_{T}$. The normal to its plane is

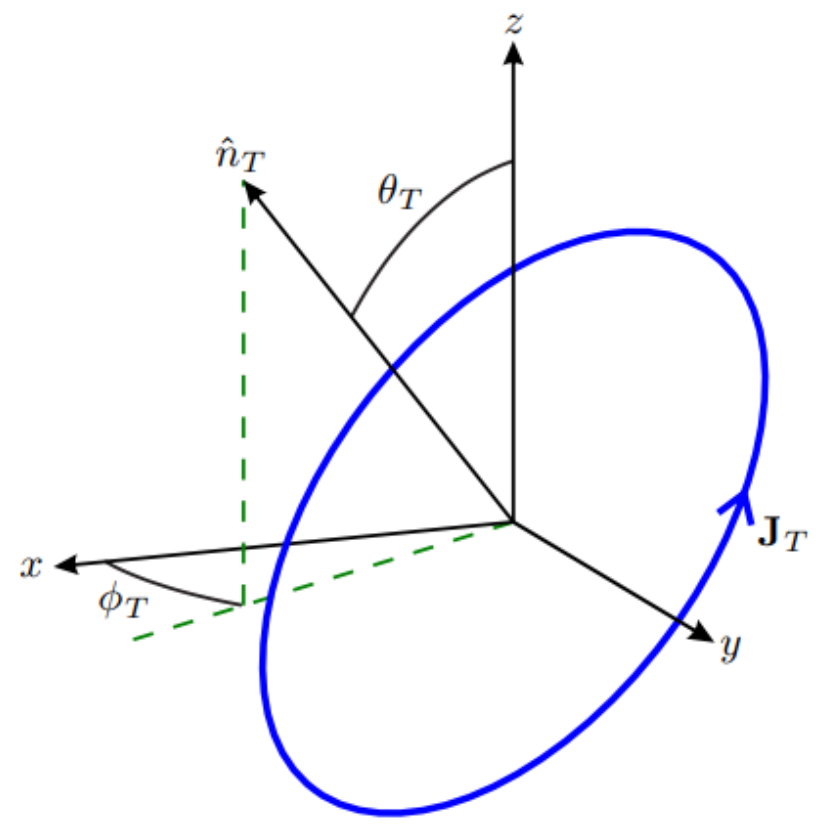

Figure 5.2: Transmitting tilted-coil antenna with current density $\mathbf{J}_{T}[1]$.

$$
\mathbf{n}_{T}=\hat{x} \sin \theta_{T} \cos \phi_{T}+\hat{y} \sin \theta_{T} \sin \phi_{T}+\hat{z} \cos \theta_{T}
$$

and the coordinates of tilted filamentary winding

$$
\mathbf{r}_{T}=\hat{\rho} \rho_{T}+\hat{z}\left[z_{T}-\rho_{T} \tan \theta_{T} \cos \left(\phi-\phi_{T}\right)\right]
$$

satisfy the equation

$$
\mathbf{n}_{T} \cdot \mathbf{r}_{T}=0,
$$

where $\rho_{T}$ and $z_{T}$ are the radial and axial position of the coil center and $\phi_{T}$ and $\theta_{T}$ are the azimuthal and elevation tilt angles, respectively.

The volumetric electric current density can be written as 


$$
\mathbf{J}_{T}=I_{T} \boldsymbol{\delta}_{T}
$$

where $I_{T}$ is the current in the winding and

$$
\begin{aligned}
\boldsymbol{\delta}_{T}=\delta\left(\rho-\rho_{T}\right) \delta\left(z-z_{T}+\rho_{T} \tan \theta_{T}\right. & \left.\cos \left(\phi-\phi_{T}\right)\right) \\
& \times\left(\hat{\phi}+\hat{z} \tan \theta_{T} \sin \left(\phi-\phi_{T}\right)\right) .
\end{aligned}
$$

\section{4}

\section{Modal Excitation from an Electric Current Source}

In the absence of magnetic sources, i.e., $\mathbf{M}_{T}=\mathbf{0}$, by taking the curl of $\mathbf{J}_{s}$ where [55]

$$
\nabla_{s}=\hat{\rho} \frac{\partial}{\partial \rho}+\hat{\phi} \frac{1}{\rho} \frac{\partial}{\partial \phi}
$$

and projecting it onto $\hat{z}$, the equations (5-11) and (5-12) leads to

$$
\begin{aligned}
\nabla_{s}^{2} H_{z}+\frac{\mu_{z}}{\mu_{s}} \frac{\partial^{2} H_{z}}{\partial z^{2}}+k^{2} H_{z} & =\frac{I_{T}}{\rho} \delta\left(\rho-\rho_{T}\right) \delta\left(z-z_{T}+\rho_{T} \tan \theta_{T} \cos \left(\phi-\phi_{T}\right)\right) \\
\nabla_{s}^{2} E_{z}+\frac{\epsilon_{z}}{\epsilon_{s}} \frac{\partial^{2} E_{z}}{\partial z^{2}}+k^{2} E_{z} & =-i \omega \mu_{s} J_{z}
\end{aligned}
$$

Given the geometries of Fig. 4.1 and Fig. 5.2, it is helpful to employ the generalized Fourier series:

$$
\begin{aligned}
& F_{j}(\rho, \phi, z)=\sum_{n=-\infty}^{\infty} \sum_{p=1}^{\infty} f_{j}\left(\rho, n, k_{z}\right) e^{i n \phi} Z_{p}\left(k_{z} z\right) \\
& f_{j}\left(\rho, n, k_{z}\right)=\frac{1}{2 \pi N_{z p}} \int_{\phi} \int_{z} F_{j}(\rho, \phi, z) e^{-i n \phi} Z_{p}^{*}\left(k_{z} z\right) d z d \phi,
\end{aligned}
$$

where

$$
\begin{aligned}
N_{z p} & =\int_{z=-z_{N+1}}^{z=-z_{1}} Z_{p}\left(k_{z} z\right) Z_{p}^{*}\left(k_{z} z\right) d z \\
Z_{p}\left(k_{z} z\right) & =\sum_{j=2}^{N+1} \operatorname{rect}\left(-z_{j-1},-z_{j}\right) \Phi_{j p}\left(k_{j z} z\right)
\end{aligned}
$$

and $\Phi_{j p}\left(k_{j z} z\right)$ is given by the equation (3-41), in which we select it depending of the TE or TM mode. Notice that $\operatorname{rect}(a, b)$ is the rectangular function: it is equal to 1 in the range $a<z<b$, and 0 otherwise. The Fourier transform given in (5-22) will be applied to each component of fields and sources, for facilitating the application of the boundary conditions. A similar procedure was done in $[1,11,49]$. Setting the wave equations (5-19) and (5-20) to homogeneous 
equations and applying (5-22), we obtain

$$
\begin{aligned}
& {\left[\frac{1}{\rho} \frac{d}{d \rho}\left(\rho \frac{d}{d \rho}\right)-\frac{n^{2}}{\rho^{2}}-\frac{\mu_{z}}{\mu_{s}} k_{z}^{2}+k^{2}\right] h_{z}\left(\rho, n, k_{z}\right)=0} \\
& {\left[\frac{1}{\rho} \frac{d}{d \rho}\left(\rho \frac{d}{d \rho}\right)-\frac{n^{2}}{\rho^{2}}-\frac{\epsilon_{z}}{\epsilon_{s}} k_{z}^{2}+k^{2}\right] e_{z}\left(\rho, n, k_{z}\right)=0}
\end{aligned}
$$

resulting in the ordinary differential equations

$$
\begin{aligned}
& {\left[\frac{1}{\rho} \frac{d}{d \rho}\left(\rho \frac{d}{d \rho}\right)+k_{\rho}^{2}-\frac{n^{2}}{\rho^{2}}\right] h_{z}\left(\rho, n, k_{z}\right)=0} \\
& {\left[\frac{1}{\rho} \frac{d}{d \rho}\left(\rho \frac{d}{d \rho}\right)+k_{\rho}^{2}-\frac{n^{2}}{\rho^{2}}\right] e_{z}\left(\rho, n, k_{z}\right)=0,}
\end{aligned}
$$

where $k_{\rho}^{2}$ is given by the equation $(3-31)$ with $\Im \mathrm{m}\left(k_{\rho}\right)>0$. Also applying the transform (5-22) to the source terms in (5-19) and (5-20), we obtain

$$
\begin{aligned}
s_{T E}\left(\rho, n, k_{z}\right) & =\frac{1}{2 \pi N_{z p}} \int_{\phi} \int_{z} S_{T E} e^{-i n \phi} Z_{p}^{*}\left(k_{z} z\right) d z d \phi \\
s_{T M}\left(\rho, n, k_{z}\right) & =\frac{1}{2 \pi N_{z p}} \int_{\phi} \int_{z} S_{T M} e^{-i n \phi} Z_{p}^{*}\left(k_{z} z\right) d z d \phi,
\end{aligned}
$$

where $S_{T E}$ and $S_{T M}$ are the source terms given by the RHS in the equations (5-19) and (5-20), respectively. We can convert the equations (5-29) and (5-30) to

$$
\begin{aligned}
s_{T E}\left(\rho, n, k_{z}\right) & =\frac{1}{2 \pi N_{z p}} \int_{\phi} e^{-i n \phi} d \phi f_{z p}\left(k_{z}\right)_{T E} \\
s_{T M}\left(\rho, n, k_{z}\right) & =\frac{1}{2 \pi N_{z p}} \int_{\phi} e^{-i n \phi} d \phi f_{z p}\left(k_{z}\right)_{T M},
\end{aligned}
$$

where

$$
\begin{aligned}
f_{z p}\left(k_{z}\right)_{T E} & =\int_{z} S_{T E} e^{-i n \phi} Z_{p}^{*}\left(k_{z} z\right) d z \\
f_{z p}\left(k_{z}\right)_{T M} & =\int_{z} S_{T M} e^{-i n \phi} Z_{p}^{*}\left(k_{z} z\right) d z
\end{aligned}
$$

Assuming the source transmitting to negative $z$ direction, we find

$$
\begin{aligned}
& Z_{p}\left(k_{z} z\right)=Z_{p}^{-}\left(k_{z} z\right)=A_{j}^{-}\left(e^{-i k_{j z} z}+\tilde{R}_{j, j+1} e^{i k_{j z}\left(z+2 z^{-}\right)}\right) \\
& Z_{p}^{*}\left(k_{z} z\right)=Z_{p}^{(-) *}\left(k_{z} z\right)=A_{j}^{(-) *}\left(e^{i k_{j z} z}+\tilde{R}_{j, j+1}^{*} e^{-i k_{j z}\left(z+2 z^{-}\right)}\right)
\end{aligned}
$$

in that $z^{-}$is given by the equation (3-42). Then, computing the integrals (5-33) 
and (5-34), we obtain

$$
\begin{aligned}
& f_{z p}\left(k_{z}\right)_{T E}=\frac{I_{T}}{\rho} A_{j}^{(-) *} \delta\left(\rho-\rho_{T}\right) \\
\times & \int_{z=-z_{N+1}}^{z=-z_{1}} \delta\left(z-z_{T}+\rho_{T} \tan \theta_{T} \cos \left(\phi-\phi_{T}\right)\right)\left(e^{i k_{j z}^{h} z}+\tilde{R}_{j, j+1}^{T E(z) *} e^{-i k_{j z}^{h} z} e^{-2 i k_{j z}^{h} z_{j}}\right) d z
\end{aligned}
$$

and

$$
\begin{aligned}
& f_{z p}\left(k_{z}\right)_{T M}=-i \omega \mu_{s} I_{T} A_{j}^{(-) *} \delta\left(\rho-\rho_{T}\right) \tan \theta_{T} \sin \left(\phi-\phi_{T}\right) \\
\times & \int_{z=-z_{N+1}}^{z=-z_{1}} \delta\left(z-z_{T}+\rho_{T} \tan \theta_{T} \cos \left(\phi-\phi_{T}\right)\right)\left(e^{i k_{j z}^{e} z}+\tilde{R}_{j, j+1}^{T M(z) *} e^{-i k_{j z}^{e} z} e^{-2 i k_{j z}^{e} z_{j}}\right) d z .
\end{aligned}
$$

By defining $z_{0}=z_{T}+\rho_{T} \tan \theta_{T} \cos \left(\phi-\phi_{T}\right)$ and assuming $-z_{N+1}<z_{0}<-z_{1}$, we can obtain

$$
f_{z p}\left(k_{z}\right)_{T E}=\frac{I_{T}}{\rho} A_{j}^{(-) *} \delta\left(\rho-\rho_{T}\right)\left(e^{i k_{j z}^{h} z_{0}}+\tilde{R}_{j, j+1}^{T E(z) *} e^{-2 i k_{j z}^{h} z_{j}} e^{-i k_{j z}^{h} z_{0}}\right)
$$

and

$$
\begin{aligned}
f_{z p}\left(k_{z}\right)_{T M}=-i \omega \mu_{s} I_{T} A_{j}^{(-) *} \delta(\rho & \left.-\rho_{T}\right) \tan \theta_{T} \sin \left(\phi-\phi_{T}\right) \\
& \times\left(e^{i k_{j z}^{e} z_{0}}+\tilde{R}_{j, j+1}^{T M(z) *} e^{-2 i k_{j z}^{e} z_{j}} e^{-i k_{j z}^{e} z_{0}}\right) .
\end{aligned}
$$

By substituting the equations above in (5-31) and (5-32) and using $\kappa_{T}=$ $k_{j z} \rho_{T} \tan \theta_{T}$, we obtain

$$
\begin{aligned}
& s_{T E}\left(\rho, n, k_{z}\right)=\frac{I_{T}}{2 \pi \rho N_{z p}} A_{j}^{(-) *} \delta\left(\rho-\rho_{T}\right) e^{-i k_{j z}^{h} z_{T}} \\
& \times \int_{-\pi}^{\pi} e^{-i n \phi}\left(e^{2 i k_{j z}^{h} z_{T}} e^{i \kappa_{T}^{h} \cos \left(\phi-\phi_{T}\right)}+\tilde{R}_{j, j+1}^{T E(z) *} e^{-2 i k_{j z}^{h} z_{j}} e^{-i \kappa_{T}^{h} \cos \left(\phi-\phi_{T}\right)}\right) d \phi
\end{aligned}
$$

and

$$
\begin{array}{r}
s_{T M}\left(\rho, n, k_{z}\right)=-\frac{i \omega \mu_{s} I_{T}}{2 \pi N_{z p}} A_{j}^{(-) *} \delta\left(\rho-\rho_{T}\right) \tan \theta_{T} e^{-i k_{j z}^{e} z_{T}} \int_{-\pi}^{\pi} \sin \left(\phi-\phi_{T}\right) e^{-i n \phi} \\
\times\left(e^{2 i k_{j z}^{e} z_{T}} e^{i \kappa_{T}^{e} \cos \left(\phi-\phi_{T}\right)}+\tilde{R}_{j, j+1}^{T M(z) *} e^{-2 i k_{j z}^{e} z_{j}} e^{-i \kappa_{T}^{e} \cos \left(\phi-\phi_{T}\right)}\right) d \phi . \quad(5-42)
\end{array}
$$

By introducing $\tilde{\phi}=\phi-\phi_{T}$, we can continue the development of the previous 
equations:

$$
\begin{aligned}
s_{T E}\left(\rho, n, k_{z}\right)=\frac{I_{T}}{2 \pi \rho N_{z p}} A_{j}^{(-) *} \delta( & \left.-\rho_{T}\right) e^{-i k_{j z}^{h} z_{T}} e^{-i n \phi_{T}} \\
& \times\left(e^{2 i k_{j z}^{h} z_{T}} I_{\# 1}^{+}+\tilde{R}_{j, j+1}^{T E(z) *} e^{-2 i k_{j z}^{h} z_{j}} I_{\# 1}^{-}\right) \\
s_{T M}\left(\rho, n, k_{z}\right)=-\frac{i \omega \mu_{s} I_{T}}{2 \pi N_{z p}} A_{j}^{(-) *} & \delta\left(\rho-\rho_{T}\right) \tan \theta_{T} e^{-i k_{j z}^{e} z_{T}} e^{-i n \phi_{T}} \\
& \times\left(e^{2 i k_{j z}^{e} z_{T}} I_{\# 2}^{+}+\tilde{R}_{j, j+1}^{T M(z) *} e^{-2 i k_{j z}^{e} z_{j}} I_{\# 2}^{-}\right),
\end{aligned}
$$

where

$$
\begin{aligned}
I_{\# 1}^{ \pm} & =\int_{-\pi-\phi_{T}}^{\pi-\phi_{T}} e^{-i n \tilde{\phi}} e^{ \pm i \kappa_{T}^{h} \cos \tilde{\phi}} d \tilde{\phi} \\
I_{\# 2}^{ \pm} & =\int_{-\pi-\phi_{T}}^{\pi-\phi_{T}} \sin \tilde{\phi} e^{-i n \tilde{\phi}} e^{ \pm i \kappa_{T}^{e} \cos \tilde{\phi}} d \tilde{\phi} .
\end{aligned}
$$

Noting that the integrand is periodic in terms of $\phi$ and the integration is over a period interval $(2 \pi)$, we can perform the integration over the symmetric interval $-\pi$ to $\pi$. Thus, the integrals reduce to

$$
\begin{aligned}
& I_{\# 1}^{ \pm}=\int_{-\pi}^{\pi} e^{-i n \phi \pm i \kappa_{T}^{h} \cos \phi} d \phi \\
& I_{\# 2}^{ \pm}=\int_{-\pi}^{\pi} \sin \phi e^{-i n \phi \pm i \kappa_{T}^{e} \cos \phi} d \phi .
\end{aligned}
$$

The integrand exponential $\exp \left( \pm i \kappa_{T} \cos \phi\right)$ is an even function in terms of $\phi$. Therefore, using the identity $\exp (-i n \phi)=\cos (n \phi)-i \sin (n \phi)$, we can verify that only the function in terms of $\cos (n \phi)$ contributes for the integration in $I_{\# 1}^{ \pm}$. Accordingly, we can write

$$
I_{\# 1}^{ \pm}=2 \int_{0}^{\pi} \cos (n \phi) e^{ \pm i \kappa_{T}^{h} \cos \phi} d \phi .
$$

Using the identity [57, p. 360 , eq. 9.1.21]

$$
J_{n}(z)=\frac{i^{-n}}{\pi} \int_{0}^{\pi} \cos (n \phi) e^{i z \cos \phi} d \phi,
$$

we can obtain

$$
I_{\# 1}^{ \pm}=2 \pi i^{n} J_{n}\left( \pm \kappa_{T}^{h}\right) .
$$

Analogously, the integral $I_{\# 2}^{ \pm}$can be written as

$$
I_{\# 2}^{ \pm}=-2 i \int_{0}^{\pi} \sin \phi \sin (n \phi) e^{ \pm i \kappa_{T}^{e} \cos \phi} d \phi .
$$


Noting that $\sin \phi \sin (n \phi)=\frac{1}{2}\{\cos [(n-1) \phi]-\cos [(n+1) \phi]\}$, we can find

$$
I_{\# 2}^{ \pm}=-i\left\{\int_{0}^{\pi} \cos [(n-1) \phi] e^{ \pm i \kappa_{T}^{e} \cos \phi} d \phi-\int_{0}^{\pi} \cos [(n+1) \phi] e^{ \pm i \kappa_{T}^{e} \cos \phi} d \phi\right\},
$$

which can be simplified by using (5-45), resulting in

$$
\begin{aligned}
I_{\# 2}^{ \pm} & =-i\left[\pi i^{n-1} J_{n-1}\left( \pm \kappa_{T}^{e}\right)-\pi i^{n+1} J_{n+1}\left( \pm \kappa_{T}^{e}\right)\right] \\
& =-i \pi i^{n}(-i)\left[J_{n-1}\left( \pm \kappa_{T}^{e}\right)+J_{n+1}\left( \pm \kappa_{T}^{e}\right)\right] \\
& =-i \pi i^{n} \frac{2 n J_{n}\left( \pm \kappa_{T}^{e}\right)}{ \pm \kappa_{T}^{e}} \\
& =2 \pi i^{n} \frac{n}{ \pm k_{j z}^{e} \rho_{T} \tan \theta_{T}} J_{n}\left( \pm \kappa_{T}^{e}\right) .
\end{aligned}
$$

Substituting (5-46) and (5-47) in (5-43) and (5-44), respectively, we can eventually obtain

$$
\begin{aligned}
s_{T E}\left(\rho, n, k_{z}\right) & =\tilde{C}_{T E}\left(\rho, n, k_{z}\right) \delta\left(\rho-\rho_{T}\right) \\
s_{T M}\left(\rho, n, k_{z}\right) & =\tilde{C}_{T M}\left(\rho, n, k_{z}\right) \delta\left(\rho-\rho_{T}\right),
\end{aligned}
$$

where

$$
\begin{aligned}
\tilde{C}_{T E}\left(\rho, n, k_{z}\right)=\frac{I_{T}}{\rho N_{z p}} C_{T E}\left(n, k_{z}\right) & A_{j}^{(-) *} e^{-i n \phi_{T}} e^{-i k_{j z}^{h} z_{T}} \\
& \times\left[e^{2 i k_{j z}^{h} z_{T}}+(-1)^{n} \tilde{R}_{j, j+1}^{T E(z) *} e^{-2 i k_{j z}^{h} z_{j}}\right]
\end{aligned}
$$

and

$$
\begin{aligned}
& \tilde{C}_{T M}\left(\rho, n, k_{z}\right)=\frac{i \omega \mu_{s} I_{T}}{N_{z p}} C_{T M}(n,\left.k_{z}\right) A_{j}^{(-) *} e^{-i n \phi_{T}} e^{-i k_{j z}^{e} z_{T}} \\
& \times\left[e^{2 i k_{j z}^{e} z_{T}}-(-1)^{n} \tilde{R}_{j, j+1}^{T M(z) *} e^{-2 i k_{j z}^{e} z_{j}}\right]
\end{aligned}
$$

with

$$
\begin{gathered}
C_{T E}\left(n, k_{z}\right)=i^{n} J_{n}\left(\kappa_{T}^{h}\right) \\
C_{T M}\left(n, k_{z}\right)=\frac{n i^{n}}{k_{j z}^{e} \rho} J_{n}\left(\kappa_{T}^{e}\right) .
\end{gathered}
$$

The transformed source terms, i.e., (5-50) and (5-51), are valid for observation points to the direction $-z$. For the source transmitting for $+z$, we can assume in (5-35) that

$$
Z_{p}\left(k_{z} z\right)=Z_{p}^{+}\left(k_{z} z\right)=A_{j}^{+}\left(e^{+i k_{j z} z}+\tilde{R}_{j, j-1} e^{-i k_{j z}\left(z+2 z^{+}\right)}\right)
$$

and, as consequence, in results from (5-50) to (5-53), we should change the 
sign of $k_{j z}^{e, h}$, substitute in the generalized reflection coefficient $j+1$ by $j-1$, and $z_{j}$ by $z_{j-1}$ from $(3-42)$.

The solutions to (5-27) and (5-28) are given by (3-39). The unknown vectors $\bar{a}$ and $\bar{b}$ will be determined by the jump and continuity conditions of the Sturm-Liouville theory of third kind [58] together with the boundary conditions of electromagnetism [2] using the source-free fields derived in Subsection 3.4 and Subsection 3.5. In a compact matrix form, we obtain:

$$
\begin{gathered}
{\left.\left[\begin{array}{l}
e_{z, n p}(\rho) \\
h_{z, n p}(\rho)
\end{array}\right]\right|_{\rho_{T}^{+}}-\left.\left[\begin{array}{l}
e_{z, n p}(\rho) \\
h_{z, n p}(\rho)
\end{array}\right]\right|_{\rho_{T}^{-}}=-\tilde{C}_{T E}\left(\rho, n, k_{z}\right)\left[\begin{array}{l}
0 \\
1
\end{array}\right]} \\
{\left.\left[\begin{array}{l}
e_{\phi, n p}(\rho) \\
h_{\phi, n p}(\rho)
\end{array}\right]\right|_{\rho_{T}^{+}}-\left.\left[\begin{array}{l}
e_{\phi, n p}(\rho) \\
h_{\phi, n p}(\rho)
\end{array}\right]\right|_{\rho_{T}^{-}}=-\tilde{C}_{T M}\left(\rho, n, k_{z}\right)\left[\begin{array}{l}
0 \\
1
\end{array}\right]}
\end{gathered}
$$

Finally, the solution will have the form

$$
\begin{gathered}
{\left[\begin{array}{l}
e_{z, n p}(\rho) \\
h_{z, n p}(\rho)
\end{array}\right]= \begin{cases}\overline{\bar{J}}_{z n}\left(k_{\rho} \rho\right) \bar{b} & \rho \leq \rho_{T} \\
\overline{\bar{H}}_{z n}^{(1)}\left(k_{\rho} \rho\right) \bar{a} & \rho>\rho_{T}\end{cases} } \\
{\left[\begin{array}{l}
e_{\phi, n p}(\rho) \\
h_{\phi, n p}(\rho)
\end{array}\right]= \begin{cases}\overline{\bar{J}}_{\phi n}\left(k_{\rho} \rho\right) \bar{b} & \rho \leq \rho_{T} \\
\overline{\bar{H}}_{\phi n}^{(1)}\left(k_{\rho} \rho\right) \bar{a} & \rho>\rho_{T}\end{cases} }
\end{gathered}
$$

The boundary conditions (5-55) and (5-56) lead to

$$
\begin{aligned}
& \overline{\bar{J}}_{z n}\left(k_{\rho} \rho\right) \bar{b}-\overline{\bar{H}}_{z n}^{(1)}\left(k_{\rho} \rho\right) \bar{a}=\tilde{C}_{T E}\left(\rho, n, k_{z}\right)\left[\begin{array}{l}
0 \\
1
\end{array}\right] \\
& \overline{\bar{J}}_{\phi n}\left(k_{\rho} \rho\right) \bar{b}-\overline{\bar{H}}_{\phi n}^{(1)}\left(k_{\rho} \rho\right) \bar{a}=\tilde{C}_{T M}\left(\rho, n, k_{z}\right)\left[\begin{array}{l}
0 \\
1
\end{array}\right]
\end{aligned}
$$

These equations were solved for $\tilde{C}_{T E}=0$ and for $\tilde{C}_{T M}=0$ in order to simplify the algebra. Thus, the vectors $\bar{a}$ and $\bar{b}$ are given by

$$
\begin{aligned}
& \bar{a}=\bar{a}_{n p}^{h}+\bar{a}_{n p}^{e} \\
& \bar{b}=\bar{b}_{n p}^{h}+\bar{b}_{n p}^{e} .
\end{aligned}
$$

With the aim to simplify the notation, the argument of the Bessel functions will be omitted in the terms of the vectors $\bar{a}$ and $\bar{b}$ given by

$$
\begin{aligned}
& \bar{a}_{n p}^{h}=\left[\overline{\bar{J}}_{\phi n} \overline{\bar{J}}_{z n}^{-1} \overline{\bar{H}}_{z n}^{(1)}-\overline{\bar{H}}_{\phi n}^{(1)}\right]^{-1}\left[-\overline{\bar{J}}_{\phi n} \overline{\bar{J}}_{z n}^{-1} \tilde{\bar{C}}_{T E}\right] \\
& \bar{a}_{n p}^{e}=\left[\overline{\bar{J}}_{\phi n} \overline{\bar{J}}_{z n}^{-1} \overline{\bar{H}}_{z n}^{(1)}-\overline{\bar{H}}_{\phi n}^{(1)}\right]^{-1} \tilde{\bar{C}}_{T M} \\
& \bar{b}_{n p}^{h}=\overline{\bar{J}}_{z n}^{-1}\left[\overline{\bar{H}}_{z n}^{(1)} a_{n p}^{h}+\tilde{\bar{C}}_{T E}\right] \\
& \bar{b}_{n p}^{e}=\overline{\bar{J}}_{z n}^{-1} \overline{\bar{H}}_{z n}^{(1)} a_{n p}^{e}
\end{aligned}
$$


where

$$
\tilde{\bar{C}}_{T M}=\tilde{C}_{T M}\left[\begin{array}{l}
0 \\
1
\end{array}\right] \quad \text { and } \quad \tilde{\bar{C}}_{T E}=\tilde{C}_{T E}\left[\begin{array}{l}
0 \\
1
\end{array}\right] .
$$

After some manipulations, we obtain

$$
\begin{aligned}
& \bar{a}_{n p}^{e}=\left[\begin{array}{c}
\left.\tilde{C}_{T M} \frac{k_{\rho}^{2} \rho_{T} J_{n}\left(k_{\rho} \rho_{T}\right)}{i \omega \epsilon_{s} k_{\rho} \rho_{T}\left[J_{n}^{\prime}\left(k_{\rho} \rho_{T}\right) H_{n}^{(1)}\left(k_{\rho} \rho_{T}\right)-J_{n}\left(k_{\rho} \rho_{T}\right) H_{n}^{\prime(1)}\left(k_{\rho} \rho_{T}\right)\right]}\right] \\
0
\end{array}\right] \\
& \bar{b}_{n p}^{e}=\left[\begin{array}{c}
\left.\tilde{C}_{T M} \frac{k_{\rho}^{2} \rho_{T} H_{n}^{(1)}\left(k_{\rho} \rho_{T}\right)}{i \omega \epsilon_{s} k_{\rho} \rho_{T}\left[J_{n}^{\prime}\left(k_{\rho} \rho_{T}\right) H_{n}^{(1)}\left(k_{\rho} \rho_{T}\right)-J_{n}\left(k_{\rho} \rho_{T}\right) H_{n}^{\prime(1)}\left(k_{\rho} \rho_{T}\right)\right]}\right] \\
0
\end{array}\right]
\end{aligned}
$$

for $\tilde{C}_{T E}=0$, and

$$
\begin{gathered}
\bar{a}_{n p}^{h}=\left[\begin{array}{c}
\tilde{C}_{T E} \frac{n k_{z}^{e} J_{n}\left(k_{\rho} \rho_{T}\right)}{i \omega \epsilon_{s} k_{\rho} \rho_{T}\left[J_{n}^{\prime}\left(k_{\rho} \rho_{T}\right) H_{n}^{(1)}\left(k_{\rho} \rho_{T}\right)-J_{n}\left(k_{\rho} \rho_{T}\right) H_{n}^{\prime(1)}\left(k_{\rho} \rho_{T}\right)\right]} \\
\tilde{C}_{T E} \frac{-J_{n}^{\prime}\left(k_{\rho} \rho_{T}\right)}{\left[J_{n}^{\prime}\left(k_{\rho} \rho_{T}\right) H_{n}^{(1)}\left(k_{\rho} \rho_{T}\right)-J_{n}\left(k_{\rho} \rho_{T}\right) H_{n}^{\prime(1)}\left(k_{\rho} \rho_{T}\right)\right]}
\end{array}\right] \\
\bar{b}_{n p}^{h}=\left[\begin{array}{c}
n k_{z}^{e} H_{n}^{(1)}\left(k_{\rho} \rho_{T}\right) \\
\tilde{C}_{T E} \frac{-H_{n}^{\prime}(1)\left(k_{\rho} \rho_{T}\right)}{i \omega \epsilon_{s} k_{\rho} \rho_{T}\left[J_{n}^{\prime}\left(k_{\rho} \rho_{T}\right) H_{n}^{(1)}\left(k_{\rho} \rho_{T}\right)-J_{n}\left(k_{\rho} \rho_{T}\right) H_{n}^{\prime(1)}\left(k_{\rho} \rho_{T}\right)\right]} \\
\tilde{C}_{T E} \frac{\left.J_{n}^{\prime}\left(k_{\rho} \rho_{T}\right) H_{n}^{(1)}\left(k_{\rho} \rho_{T}\right)-J_{n}\left(k_{\rho} \rho_{T}\right) H_{n}^{\prime(1)}\left(k_{\rho} \rho_{T}\right)\right]}{\left[J_{n}\right.}
\end{array}\right]
\end{gathered}
$$

for $\tilde{C}_{T M}=0$.

By summing the terms (5-68)-(5-71) and using the Wronskian of Bessel's equation [59]

$$
J_{n}^{\prime}\left(k_{\rho} \rho_{T}\right) H_{n}^{(1)}\left(k_{\rho} \rho_{T}\right)-J_{n}\left(k_{\rho} \rho_{T}\right) H_{n}^{\prime(1)}\left(k_{\rho} \rho_{T}\right)=-\frac{2 i}{\pi k_{\rho} \rho_{T}},
$$

we can find the amplitudes (5-61) and (5-62) for anisotropic media in a compact form:

$$
\bar{c}_{n p}^{ \pm}=-\frac{i \pi}{2}\left[\begin{array}{c}
\left(n k_{z}^{e} \tilde{C}_{T E}+k_{\rho}^{2} \rho_{T} \tilde{C}_{T M}\right) \frac{i}{\omega \epsilon_{s}} B_{n}\left(k_{\rho} \rho_{T}\right) \\
\tilde{C}_{T E} k_{\rho} \rho_{T} B_{n}^{\prime}\left(k_{\rho} \rho_{T}\right)
\end{array}\right] .
$$

In the particular case of an isotropic media, we have

$$
J_{n}\left(\kappa_{T}^{h}\right)=J_{n}\left(\kappa_{T}^{e}\right)=J_{n}\left(\kappa_{T}\right)
$$

and the equation (5-73) can be transformed to the solution presented in 
[49], i.e.,

$$
\bar{c}_{n p}^{ \pm}=-\frac{i \pi}{2} \tilde{C}_{T E}\left[\begin{array}{c}
\frac{i \omega \mu_{s} n}{k_{z}^{e}} B_{n}\left(k_{\rho} \rho_{T}\right) \\
k_{\rho} \rho_{T} B_{n}^{\prime}\left(k_{\rho} \rho_{T}\right)
\end{array}\right]
$$

where $B_{n}=J_{n}$ for $\bar{c}_{n p}^{+}=\bar{a}$ and $B_{n}=H_{n}^{(1)}$ for $\bar{c}_{n p}^{-}=\bar{b}$.

Finally, the fields can be determined by the inverse transform given in $(5-21)$.

\section{5}

\section{Numerical Implementation and Analysis}

In order to validate the mathematical formulation presented along this chapter, in this section we present the results of a simple example to verify the contribution of an electric current source and its influence on electromagnetic fields.

For the implementation of the coil source, we model a suitable horizontal antenna for solving TE fields, in which $n=0$ and $\phi_{T}=0$. The propagation direction of the transmitted waves is towards negative axial domain with a position of the source at $z=-0.85 \mathrm{~m}$ and a constant electric current of $1 \mathrm{~A}$. The medium approached is similar to that of Section 4.5.1.1 for a homogeneous medium into a parallel plate waveguide as shown in Fig. 4.3. The operation frequency in this example is $200 \mathrm{kHz}$ frequency. We assume values of electrical permittivity and magnetic permeability equal to those of vacuum $\left(\epsilon_{0}\right.$ and $\left.\mu_{0}\right)$. Therefore, the relative dielectric and magnetic constants $\left(\epsilon_{r}\right.$ and $\left.\mu_{r}\right)$ are unity. We consider the lossy medium described by its electrical conductivity with value $0.1 \mathrm{~S} / \mathrm{m}$.

In Fig. 5.3 and Fig. 5.4 the absolute values of the azimuthal component amplitude of the electric field due to a unit electric current excitation are plotted. In Fig. 5.3 three cases are presented for 10, 20 and 30 modes. We can appreciate the field convergence as the number of assessed modes increases. In order to corroborate the convergence, Fig. 5.4 shows the results for a greater number of modes. In both figures we can also notice the source position at $z=-0.85 \mathrm{~m}$ as we simulate with more modes. Moreover, a greater field intensity around this point can be easily recognized. 


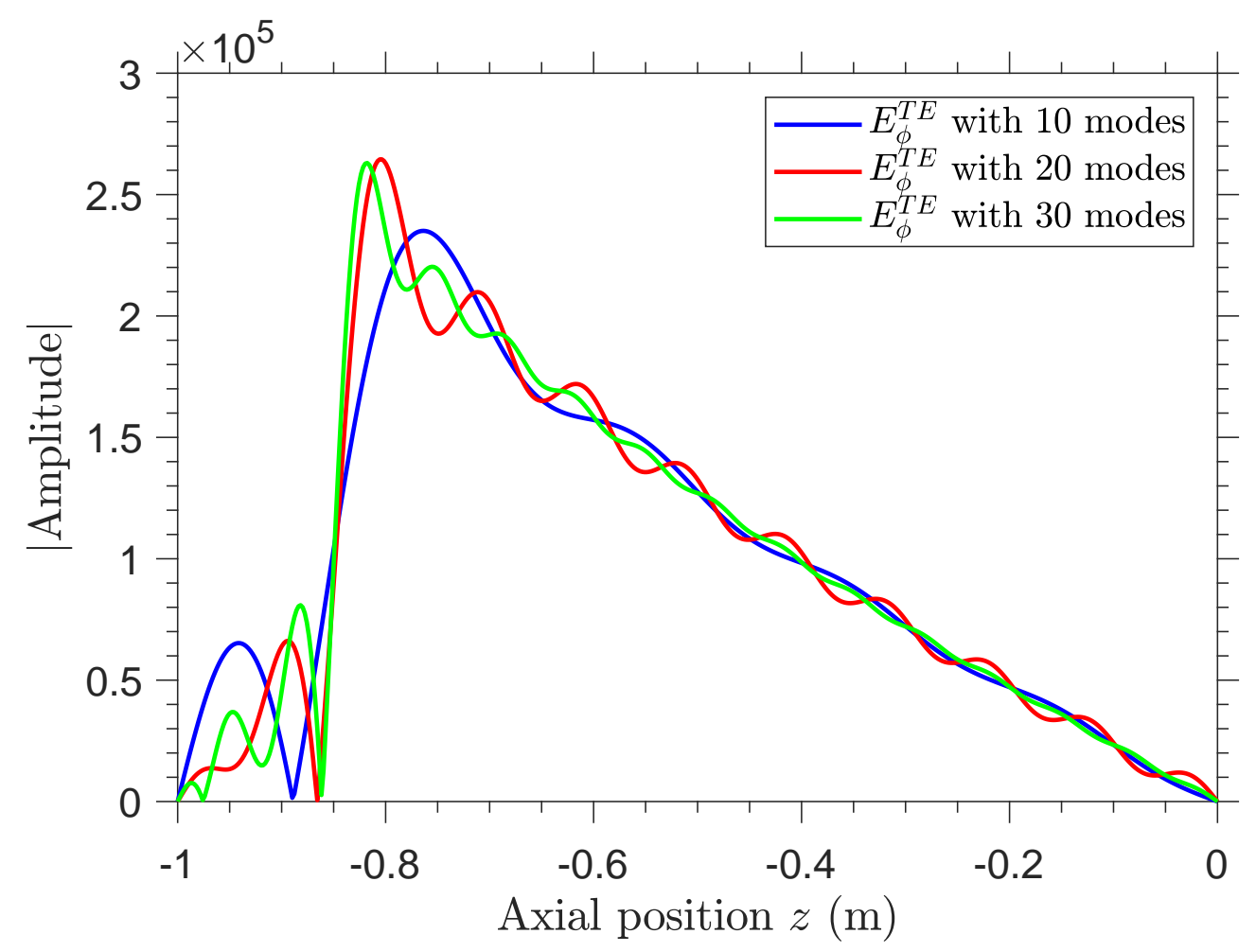

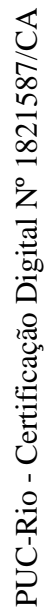

Figure 5.3: Absolute values for 10, 20 and 30 modes of the azimuthal component of the electric field $\left(E_{\phi}\right)$ for TE mode in a lossy homogeneous and isotropic medium confined by a parallel plate waveguide.

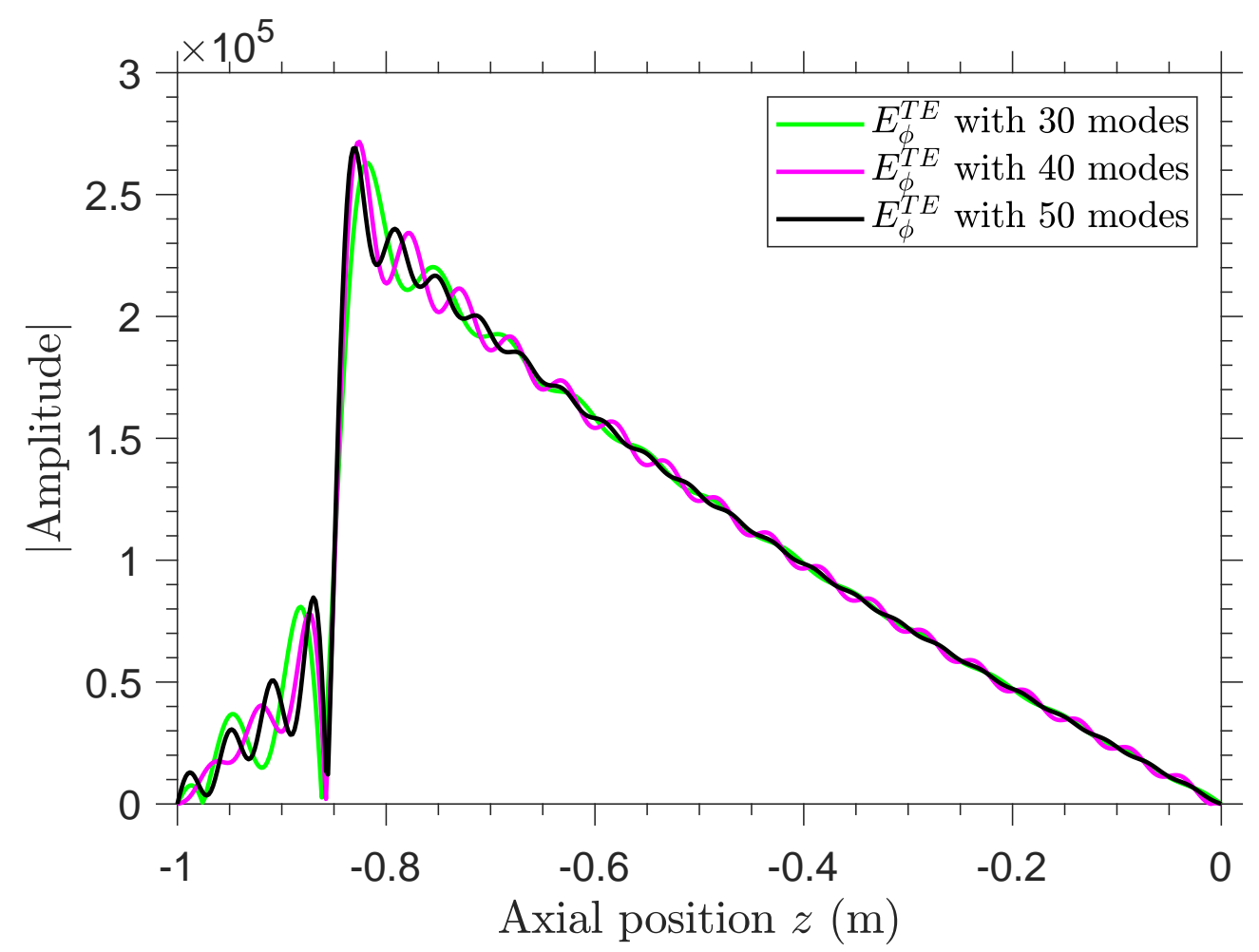

Figure 5.4: Absolute values Amplitude for 30, 40 and 50 modes of the azimuthal component of the electric field $\left(E_{\phi}\right)$ for TE mode in a lossy homogeneous and isotropic medium confined by a parallel plate waveguide. 


\section{6}

\section{Conclusions and Suggestions for Future Research}

Electromagnetic wireless telemetry systems play an important role in geophysical exploration. These systems require increasingly faster and more accurate solutions to obtain a suitable formation evaluation. Numerical methods based on spatial discretization demand a high cost in terms of computational time and RAM usage for analyzing the electromagnetic wave propagation in complex environments. In order to avoid the traditional highcost analysis, this work presents an accurate semi-analytical model to analyze the electromagnetic fields propagation in planarly-layered anisotropic media conforming to the cylindrical coordinate system. This approach allows a robust and numerically efficient computational method for modeling complex geophysical sensors.

We have introduced a mathematical formalism in Chapter 3 for solving the electric and magnetic fields in form of a sum of discrete fields through modal eigenfunction series. Our approach allows us to computing the field solutions once for any position of source or receptor. Alternative semi-analytical solutions studied before required the entire computation of associated Sommerfeld's integral at each new observation point, $c p$. the works in $[2,60]$. In addition, our solutions does not rely on the spacial discretization if the longitudinal domain, requiring a large number of modal eigenvalues until convergence be evidenced, $c p$. the works in [11,31]. The modal fields are represented by a linear combination of decoupled TE and TM modes (with respect to the longitudinal direction) due to axially-stratified media. This representation is more is less cumbersome compared to the works presented in $[1,53]$, where hybrid modes are required. Also, numerical issues associated to large-argument Bessel functions [61] are also mitigated when the presented formalism is used as a basis for a mode-matching enforcement.

The effects of the axial stratifications in the formation are analyzed in the Chapter 4, where the boundary-value problem is described by using a computationally-efficient recursive formulation via generalized Fresnel coefficients for accounting multiple reflections in anisotropic media. Such coefficients allowed is to incorporate multiple layers along the axial domain, and generalized the former ones used for isotropic backgrounds presented in 
[2]. A series of numerical simulations where presented to validate our method by an exhaustive verification of the boundary-conditions enforcement.

In Chapter 5, a mathematical formulation for expanding an electrical coil-source and to find its contribution to the modal fields derived in Chapter 4 was presented. The modal amplitudes due to the source were computed via the Sturm-Liouville theory. Some results were presented in order to validate our method as well as the field convergence as verified.

In this dissertation we have explored some new semi-analytical approaches for the modeling of electromagnetic propagation in axially-stratified geophysical scenarios analogous to those of the Brazilian Pre-Salt. Another potential application of this study is in the so-called IOUT, especially for precision agriculture where the methods showed here can be employed in soil characterization via electromagnetic sensing. As future works, we suggest the extension of the proposed solution into mode-matching-based approaches for accounting the effects of radial stratifications. 


\section{Bibliography}

[1] G. S. Rosa, "Pseudo-analytical modeling for electromagnetic well-logging tools in complex geophysical formations," Ph.D. dissertation, Dept. Elect. Eng., Pontifical Catholic University of Rio de Janeiro, Rio de Janeiro, RJ, Brazil, 2017.

[2] W. C. Chew, Waves and Fields in Inhomogeneous Media. New York, NY, USA: John Wiley \& Sons, 1995.

[3] G. S. Rosa and J. R. Bergmann, "Pseudo-analytical modeling for the electromagnetic propagation in stratified cylindrical structures," IEEE Antennas Wireless Propag. Lett., vol. 15, pp. 344-347, 2016.

[4] S. Zeng, D. Li, D. Wilton, and J. Chen, "Fast and accurate simulation of electromagnetic telemetry in deviated and horizontal drilling," Journal of Petroleum Science and Engineering, vol. 166, 2018.

[5] A. Salam, M. C. Vuran, X. Dong, C. Argyropoulos, and S. Irmak, "A theoretical model of underground dipole antennas for communications in internet of underground things," IEEE Transactions on Antennas and Propagation, vol. 67, no. 6, pp. 3996-4009, 2019.

[6] Y. Bai, Q. Zhan, H. Wang, T. Chen, Q. He, and D. Hong, "Calculation of tilted coil voltage in cylindrically multilayered medium for well-logging applications," IEEE Access, vol. 8, pp. 30081-30 091, 2020.

[7] K. Sainath and F. L. Teixeira, "Analysis of triaxial well-logging sensors in layered anisotropic earth formations," in 2016 10th European Conference on Antennas and Propagation (EuCAP), 2016, pp. 1-5.

[8] J. Lovell and W. Chew, "Response of a point source in a multicylindrically layered medium," IEEE Trans. Geosci. Remote Sens., vol. 25, pp. 850-858, Nov. 1987.

[9] A. Q. Howard, "Petrophysics of magnetic dipole fields in an anisotropic earth," IEEE Trans. Antennas Propag., vol. 48, no. 9, pp. 1376-1383, Sep. 2000. 
[10] W. Chew, E. Michielssen, J. M. Song, and J. M. Jin, Fast and Efficient Algorithms in Computational Electromagnetics. USA: Artech House, Inc., 2001.

[11] Y.-K. Hue, "Analysis of electromagnetic well-logging tools," Ph.D. dissertation, Dept. Elect. Eng., The Ohio State University, Columbus, OH, USA, 2006.

[12] H. O. Lee, F. L. Teixeira, L. E. San Martin, and M. S. Bittar, "Numerical modeling of eccentered Iwd borehole sensors in dipping and fully anisotropic earth formations," IEEE Transactions on Geoscience and Remote Sensing, vol. 50, no. 3, pp. 727-735, 2012.

[13] R. Rahmatillah, Chairunnisa, and A. Munir, "Numerical analysis for wave propagation in circular waveguide using cylindrical coordinate system-based fdtd method," in 2014 International Conference of Advanced Informatics: Concept, Theory and Application (ICAICTA), 2014, pp. 209-213.

[14] L. Hui, S. Yi-ze, and Z. Xi-fang, "Numerical simulation of resistivity LWD tool based on higher-order vector finite element," J. Petrol. Explor. Prod. Technol., vol. 6, no. 3, pp. 533-543, Sep. 2015.

[15] C. Liu, Theory of Electromagnetic Well Logging. Amsterdam, Netherlands: Elsevier, 1st edition, 2017.

[16] H. Liu, Principles and Applications of Well Logging, ser. Springer Mineralogy. Berlin, Germany: Springer, 2017.

[17] M. S. Novo, L. C. da Silva, and F. L. Teixeira, "Analysis of electromagnetic well logging tools for oil and gas exploration using finite volume techniques," in 2007 SBMO/IEEE MTT-S International Microwave and Optoelectronics Conference, 2007, pp. 634-638.

[18] M. S. Novo, "Numerical analysis of electromagnetic well-logging tools by using finite volume methods," Ph.D. dissertation, Dept. Elect. Eng., Pontifical Catholic University of Rio de Janeiro, Rio de Janeiro, RJ, Brazil, 2007, in Portuguese. [Online]. Available: http://doi.org/10.17771/PUCRio.acad.11478

[19] Wen Xun Zhang, "A review of computational electromagnetics in china," IEEE Antennas and Propagation Magazine, vol. 39, no. 3, pp. 44-50, 1997.

[20] W. C. Chew, Z. Nie, Q. H. Liu, and B. Anderson, "An efficient solution for the response of electrical well logging tools in a complex environment," IEEE Trans. Geosci. Remote Sens., vol. 29, no. 2, pp. 308-313, Mar. 1991. 
[21] Q.-H. Liu, "Electromagnetic field generated by an off-axis source in a cylindrically layered medium with an arbitrary number of horizontal discontinuities," Geophysics, vol. 58, no. 5, pp. 616-625, May 1993.

[22] Y. Guéguen, V. Palciauskas, and M. Yourcenar, Introduction to the Physics of Rocks. Princeton University Press, 1994. [Online]. Available: https://books.google.com.br/books?id=fCP5qyRyX-oC

[23] G.-S. Liu, F. L. Teixeira, and G.-J. Zhang, "Analysis of directional logging tools in anisotropic and multieccentric cylindrically-layered earth formations," IEEE Trans. Antennas Propag., vol. 60, no. 1, pp. 318-327, Jan. 2012.

[24] G. S. Rosa, J. R. Bergmann, and S. R. Zang, "Improvements on the search modal propagation constants in lossy circular waveguides," IEEE Antennas Wireless Propag. Lett., vol. 13, pp. 754-757, 2014.

[25] G. S. Rosa and J. R. Bergmann, "Electromagnetic propagation along lossy anisotropic and radially stratified cylindrical structures," in Loughborough Antennas and Propagation Conference (LAPC), Loughborough, UK, Nov. 2014, pp. 155-159.

[26] G. S. Rosa, J. R. Bergmann, and F. L. Teixeira, "Axial mode-matching technique for analysis of directional well-logging sensor tools," in IEEE International Symposium on Antennas and Propagation/USNC-URSI National Radio Science, Fajardo, Puerto Rico, Jun. 26-Jul. 1st 2016, pp. 2041-2042.

[27] _ - "Mode-matching modeling of low-frequency wireless telemetry in deep oil fields," in 2017 11th European Conference on Antennas and Propagation (EuCAP), Paris, France, Mar. 19-24 2017, to appears.

[28] G. S. Rosa, M. S. Canabarro, J. R. Bergmann, and F. L. Teixeira, "A comparison of two numerical mode-matching methodologies for the analysis of inhomogeneous media with radial and vertical stratifications," IEEE Transactions on Antennas and Propagation, vol. 66, no. 12, pp. 7499-7504, 2018.

[29] G. S. Rosa, J. R. Bergmann, and F. L. Teixeira, "A perturbation method to model electromagnetic well-logging tools in curved boreholes," IEEE Transactions on Geoscience and Remote Sensing, vol. 56, no. 4, pp. 19791993, 2018.

[30] _ - "Analysis of transient electromagnetic field propagation in well-logging environments via an efficient mode-matching technique," in 2019 13th European Conference on Antennas and Propagation (EuCAP), 2019, pp. 1-5. 
[31] M. S. Canabarro, "Application of numerical mode-matching method in the study of well logging oil with multiple knots," Ph.D. dissertation, Dept. Elect. Eng., Pontifical Catholic University of Rio de Janeiro, Rio de Janeiro, RJ, Brazil, 2016, in Portuguese.

[32] T. Hagiwara, E. J. Banning, R. M. Ostermeier, and S. M. Haugland, "Effects of mandrel, borehole, and invasion for tilt-coil antennas," in SPE 78th Ann. Tech. Conf. Exhibit., Denver, CO, USA, 5-8 Oct. 2003.

[33] S. Yang, D. Hong, W. Huang, and Q. H. Liu, "A stable analytic model for tilted-coil antennas in a concentrically cylindrical multilayered anisotropic medium," IEEE Geoscience and Remote Sensing Letters, vol. 14, no. 4, pp. 480-483, 2017.

[34] G. S. Rosa, J. R. Bergmann, and F. L. Teixeira, "Pseudo-analytical modeling of tilted-coil antennas in anisotropic geophysical formations," in $17^{\circ}$ SBMO - Simpósio Brasileiro de Micro-ondas e Optoeletrônica - 12 CBMag Congresso Brasileiro de Eletromagnetismo, Porto Alegre, Brazil, Jul. 25-29 2016.

[35] — - "Pseudoanalytical modeling of electromagnetic well-logging sensors inside directional wells," in 32nd International Union of Radio Science General Assembly and Scientific Symposium, Montreal, Canada, Aug. 19-26 2017, submitted.

[36] M. Sadiku, Elementos de Electromagnetismo, ser. Oxford / Alfaomega. Oxford University Press Mexico, Third Edition, 2010.

[37] W. Gibson, The Method of Moments in Electromagnetics. CRC Press / Taylor \& Francis Group, Second edition, 2015.

[38] K. Kunz and R. Luebbers, The Finite Difference Time Domain Method for Electromagnetics, 1st ed. CRC Press / Taylor and Francis, 1993.

[39] F. L. Teixeira and W. C. Chew, "Finite-difference computation of transient electromagnetic waves for cylindrical geometries in complex media," IEEE Trans. Geosci. Remote Sens., vol. 38, no. 4, pp. 1530-1543, Jul. 2000.

[40] Y.-K. Hue and F. L. Teixeira, "FDTD simulation of MWD electromagnetic tools in large-contrast geophysical formations," IEEE Trans. Magn., vol. 40, pp. 1456-1459, Mar. 2004.

[41] A. Taflove and S. C. Hagness, Computational electrodynamics: the finitedifference time-domain method, 3rd ed. Norwood: Artech House, 2005. 
[42] H. O. Lee and F. L. Teixeira, "Cylindrical fdtd analysis of Iwd tools through anisotropic dipping-layered earth media," IEEE Transactions on Geoscience and Remote Sensing, vol. 45, no. 2, pp. 383-388, 2007.

[43] K. Panayappan, S. Jain, R. Mittra, and J. C. Goswami, "Modeling logging while drilling systems at low frequencies using the finite difference time domain (fdtd)," in 2014 IEEE Antennas and Propagation Society International Symposium (APSURSI), 2014, pp. 500-501.

[44] R. Coccioli, T. Itoh, G. Pelosi, and P. P. Silvester, "Finite-element methods in microwaves: a selected bibliography," IEEE Antennas and Propagation Magazine, vol. 38, no. 6, pp. 34-48, 1996.

[45] Y. Zhu, Multigrid Finite Element Methods for Electromagnetic Field Modeling. John Wiley \& Sons, Ltd, 032006.

[46] J. Jin, The Finite Element Method in Electromagnetics, 3rd ed. Wiley-IEEE Press, 2014.

[47] Z. Q. Zhang, X. Zhang, and L. Mu, "Simulation of electromagnetic loggingwhile-drilling tools using vector finite element methods," in 2011 IEEE International Symposium on Antennas and Propagation (APSURSI), 2011, pp. 2499-2502.

[48] F. L. Teixeira and J. R. Bergmann, "Moment-method analysis of circularly symmetric reflectors using bandlimited basis functions," IEE Proceedings Microwaves, Antennas and Propagation, vol. 144, no. 3, pp. 179-183, 1997.

[49] T. Hagiwara, E. Banning, R. Ostermeier, and M. Haugland, "Effects of mandrel, borehole, and invasion for tilt-coil antennas," SPE Reservoir Evaluation \& Engineering - SPE RESERV EVAL ENG, vol. 8, pp. 255-263, 2005.

[50] G. S. Rosa, J. R. Bergmann, and S. R. Zang, "Electromagnetic analysis of wireless telemetry for oil fields," in 2013 SBMO/IEEE MTT-S International Microwave Optoelectronics Conference (IMOC), Rio de Janeiro, Brazil, Jul. 4-7 2013, pp. 1-4.

[51] G. S. Rosa, M. S. Canabarro, J. R. Bergmann, and F. L. Teixeira, "A comparison of numerical mode-matching techniques for the analysis of welllogging tools," in 2017 International Applied Computational Electromagnetics Society (ACES) Symposium, Firenze, Italy, Mar. 26-30 2017, to appears.

[52] D. Fink and D. Christiansen, Manual de Ingeiería Electrónica. España: Edit. McGraw-Hill, 1992, vol. I Fundamentos para la Ingeniería Electrónica. 
[53] G. S. Rosa, J. R. Bergmann, and F. L. Teixeira, "A robust mode-matching algorithm for the analysis of triaxial well-logging tools in anisotropic geophysical formations," IEEE Trans. Geosci. Remote Sens., vol. 55, no. 5, pp. 2534-2545, May 2017.

[54] J. Kong, Electromagnetic Wave Theory. New York, NY, USA: John Wiley \& Sons, 1986.

[55] R. F. Harrington, Time-harmonic electromagnetic fields. New York, NY, USA: McGraw-Hill, 1961.

[56] M. Bittar, "Electromagnetic wave resistivity tool having a tilted antenna for determining the horizontal and vertical resistivities and relative dip angle in anisotropic earth formations," U.S. Patent 6,163,155, Dec. 2000.

[57] M. Abramowitz and I. A. Stegun, Handbook of Mathematical Functions: With Formulas, Graphs, and Mathematical Tables. New York, NY, USA: Dover Publications, 1964.

[58] D. G. Dudley, Mathematical Foundations for Electromagnetic Theory. New York, NY, USA: IEEE Press, 1994.

[59] "NIST Digital Library of Mathematical Functions," http://dlmf.nist.gov/, f. W. J. Olver, A. B. Olde Daalhuis, D. W. Lozier, B. I. Schneider, R. F. Boisvert, C. W. Clark, B. R. Miller and B. V. Saunders, eds.

[60] J. A. Rams, "Electromagnetic wave propagation in biaxially anisotropic media with azimuthal symmetry for modeling wireless telemetry in deep oil well," Master's thesis, Dept. Elect. Eng., Pontifical Catholic University of Rio de Janeiro, Rio de Janeiro, RJ, Brazil, 2019.

[61] G. S. Rosa, J. R. Bergmann, and F. L. Teixeira, Full-Wave Pseudoanalytical Methods for Cylindrically Symmetric Electromagnetic Structures: Applications in Sensing and Telemetry for Geophysical Exploration. World Scientific Publishing Co Pte Ltd, 2020, ch. 10, pp. 385-431. 